\title{
WestVirginiaUniversity
}

THE RESEARCH REPOSITORY @ WVU

Graduate Theses, Dissertations, and Problem Reports

2007

\section{Biolayer modeling and optimization for the SPARROW biosensor}

Ke Feng

West Virginia University

Follow this and additional works at: https://researchrepository.wvu.edu/etd

\section{Recommended Citation}

Feng, Ke, "Biolayer modeling and optimization for the SPARROW biosensor" (2007). Graduate Theses, Dissertations, and Problem Reports. 2580.

https://researchrepository.wvu.edu/etd/2580

This Dissertation is protected by copyright and/or related rights. It has been brought to you by the The Research Repository @ WVU with permission from the rights-holder(s). You are free to use this Dissertation in any way that is permitted by the copyright and related rights legislation that applies to your use. For other uses you must obtain permission from the rights-holder(s) directly, unless additional rights are indicated by a Creative Commons license in the record and/ or on the work itself. This Dissertation has been accepted for inclusion in WVU Graduate Theses, Dissertations, and Problem Reports collection by an authorized administrator of The Research Repository @ WVU.

For more information, please contact researchrepository@mail.wvu.edu. 


\title{
BIOLAYER MODELING AND OPTIMIZATION FOR THE SPARROW BIOSENSOR
}

\author{
By \\ Ke Feng \\ Dissertation Submitted to the \\ Eberly College of Arts and Sciences \\ at West Virginia University \\ in partial fulfillment of the requirements \\ for the degree of \\ Doctor of Philosophy \\ In \\ Physics
}

Committee members:

Dr. Nancy C. Giles, Co-advisor

Dr. Lawrence A. Hornak, Co-advisor

Dr. Martina E. Bachlechner

Dr. Larry E. Halliburton

Dr. Thomas H. Myers

Department of Physics

Morgantown, West Virginia

2007

Keywords: Biosensor, Biolayer, Transducer, Coupled waveguides, Random surface loading, Scattering 


\section{Abstract \\ Biolayer Modeling and Optimization for the Sparrow Biosensor}

\section{Ke Feng}

Biosensor direct detection of molecular binding events is of significant interest in applications from molecular screening for cancer drug design to bioagent detection for homeland security and defense. The Stacked Planar Affinity Regulated Resonant Optical Waveguide (SPARROW) structure based on coupled waveguides was recently developed to achieve increased sensitivity within a fieldable biosensor device configuration. Under ideal operating conditions, modification of the effective propagation constant of the structure's sensing waveguide through selective attachment of specific targets to probes on the waveguide surface results in a change in the coupling characteristics of the guide over a specifically designed interaction length with the analyte. Monitoring the relative power in each waveguide after interaction enables 'recognition' of those targets which have selectively bound to the surface. However, fabrication tolerances, waveguide interface roughness, biolayer surface roughness and biolayer partial coverage have an effect on biosensor behavior and achievable limit of detection (LOD). In addition to these influences which play a role in device optimization, the influence of the spatially random surface loading of molecular binding events has to be considered, especially for low surface coverage. In this dissertation an analytic model is established for the SPARROW biosensor which accounts for these nonidealities with which the design of the biosensor can be guided and optimized. For the idealized case of uniform waveguide transducer layers and biolayer, both theoretical simulation (analytical expression) and computer simulation (numerical calculation) are completed. For the nonideal case of an inhomogeneous transducer with nonideal waveguide and biolayer surfaces, device output power is affected by such physical influences as surface scattering, coupling length, absorption, and percent coverage of binding events. Using grating and perturbation techniques we explore the influence of imperfect surfaces and random surface loading on scattering loss and coupling length. Results provide a range of achievable limits of detection in the SPARROW device for a given target size, surface loading, and detectable optical power. 


\section{Acknowledgements}

I would like to acknowledge people for helping me during my doctoral studies. Firstly, I would especially like to thank my advisor, Dr. Lawrence Hornak and Dr. Nancy Giles, for their generous time and commitment. Throughout my doctoral work they encouraged me to develop independent thinking and research skills. They continually stimulated my analytical thinking and greatly assisted me with scientific writings. Secondly, I am also very grateful for having an exceptional doctoral committee and wish to thank Dr. Thomas Myers, Dr. Larry Halliburton, and Dr. Martina Bachlechner for their support and encouragement.

In addition I would also like to thank members in my research group for their assistance, generosity, support and their advice. They are Dr. Dimitris Korakakis, Dr. Aaron Timperman, Dr. Mim Soo Lim, Dr. Kolin Brown, Pavan Samudrala, Josh Nightingale and Praneeta Poloju. Especially, Dr. Mim Soo Lim gave me a lot of help in biolayer design, grown and measurements.

Finally, I would like to extent many thanks to my friends and family for their constant help and support throughout my career. 


\section{Index of Contents}

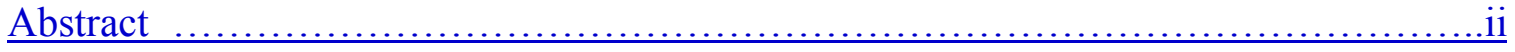

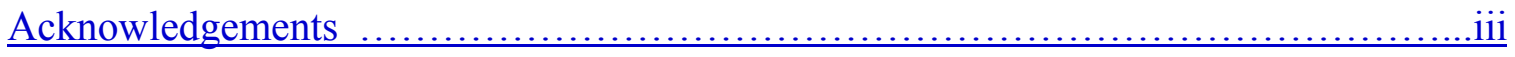

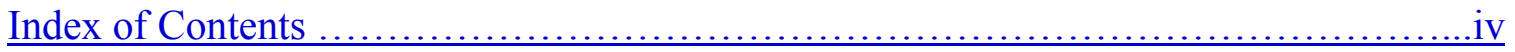

Chapter 1: Introduction ...................................................

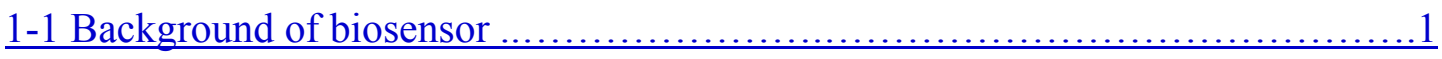

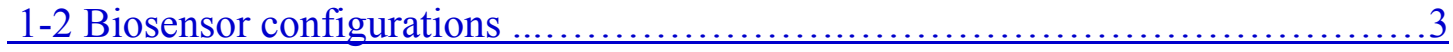

1-2-1 Amperometric biosensors and potentiometric biosensors …............4

1-2-2 Optical biosensors .............................................6

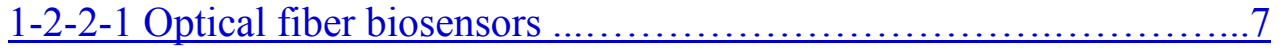

1-2-2-2 Surface plasmon resonance (SPR) biosensors ..................8

1-3 Biosensor applications .................................................... 10

1-3-1 Clinical applications ..............................................11

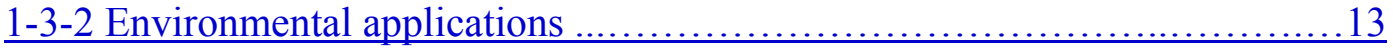

1-3-3 Process/products control .........................................14

1-4 Resonant integrated optical waveguide biosensor ...........................14

1-4-1 Structure and Fabrications …......................................15

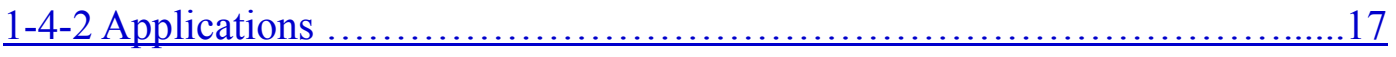

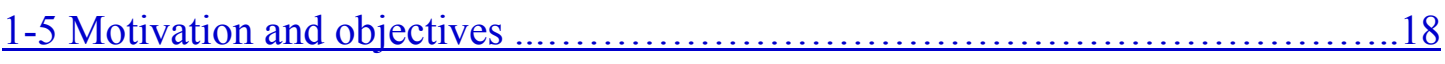

Chapter 2: Principles of SPARROW biosensor waveguide ….....................20

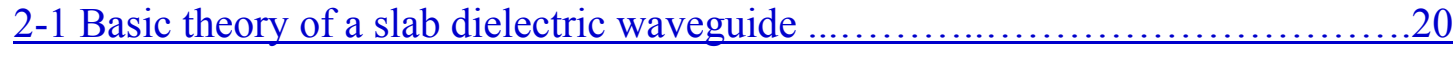

2-1-1 Basic electromagnetic approach ...............................21

2-1-2 Light behavior at the slab dielectric waveguide interface ….....................23

2-1-2-1 Boundary conditions between two dielectric interface …..........23

2-1-2-2 Reflection and refraction at the interface ........................24

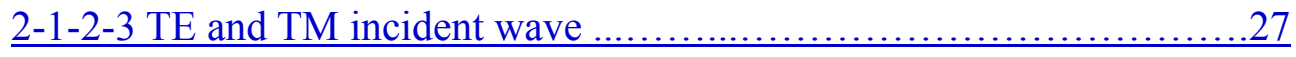

2-1-3 Light propagation in a dielectric waveguide …....................28

2-1-3-1 Evanescent waves .............................................28

2-1-3-2 TE and TM modes in symmetric dielectric waveguide ……............29

2-1-3-3 TE and TM modes in asymmetric dielectric waveguide ..................31

2-1-3-4 Effective refractive index and dispersion relation in waveguide ....33 
2-2 Mode coupling in a directional coupler .35

2-2-1 Coupling coefficient ………………………………..................................

2-2-2 Synchronous directional coupler …………………...............................

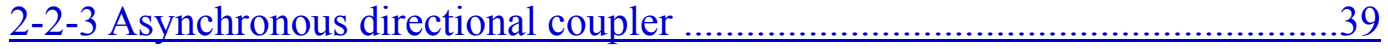

2-2-4 Non-orthogonal coupled mode theory ……………......................................40

Chapter 3: Optical modeling for ideal SPARROW biosensor structure …………….........43

3-1 Beam propagation method ...............................................................................43

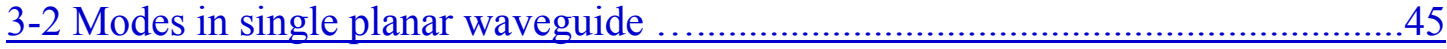

3-3 Modes and light propagation in coupled waveguide ……………………………...48

3-4 Biolayer influence for light propagation in coupled waveguide ………...............49

3-5 Mathematical model for ideal structure - numerical results ...............................58

Chapter 4: Biolayer construction ..................................................................................61

4-1 Biolayer design .................................................................................................61

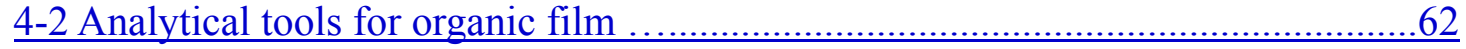

4-2-1 Ellipsometry ………………………………………...................................63

4-2-2 Atomic force microscopy (AFM) ………………....................................65

4-2-3 Contact-angle measurement ….....................................................68

4-2-4 Fourier Transform Infrared (FTIR) spectrometer .......................................69

4-3 Experiment results and analysis .............................................................. 72

4-3-1 Bare sapphire and alumina …………....................................................72

4-3-2 Self assemble monolayer ..............................................................................74

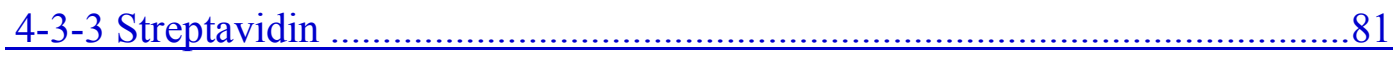

4-3-4 Biotin conjugated microsphere beads ………….....................................84

Chapter 5: Non-ideal SPARROW biosensor modeling ....................................................87

5-1 Scattering loss in waveguide …………………………………………………...... 87

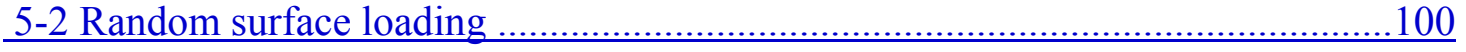

5-3 Issues Impacting the Limit of Detection (LOD) of the SPARROW biosensor ...113

Chapter 6: Summary and discussion ........................................................................119

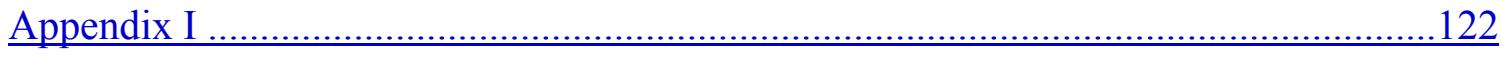

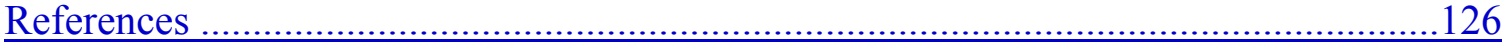




\section{Chapter 1}

\section{Introduction}

Biosensors, one special kind of sensor, can be considered as a device which is developed from any basic sensor by adding a biological component. The general aim of biosensors is to detect biologically relevant information. Generally, biosensors produce either discrete or continuous signals (e.g., electronic signals, optical signals) that respond to a single analyte (i.e., the specific targeted sample) or a related group of analytes (Turner et al., 1987). Since the differentiation between biosensors and chemical sensors is often difficult, there have been several different definitions for the term 'biosensor'. These definitions emphasize either the role of the device or the nature of the recognition process involved in generating the selective signal. One definition of a biosensor is a sensing device that incorporates a biological material such as an enzyme, antibody, or a bacterium. This definition emphasizes the signal generating process. Another definition for a biosensor, emphasizing the application, is a device which senses a species which is of a particular biological origin. A more general definition (Turner et al., 1987) is that a biosensor is 'a compact analytical device incorporating a biological or biologicallyderived sensing element either integrated within or intimately associated with a physicochemical transducer'. In 1992, the International Union of Pure and Applied Chemistry (IUPAC) committee gave the biosensor a standard definition as: 'A device that uses specific biochemical reactions mediated by isolated enzymes, immunosystems, tissues, organelles or whole cells to detect chemical compounds, usually by electrical, thermal or optical signals' (Nagel et al., 1992).

\section{1-1 Background of biosensor}

Table 1 (Newman, 2001) shows the major landmarks in biosensor development. The earliest biosensor device was invented in 1962, when Clark and Lyons were able to detect glucose by measuring the concentration of $\mathrm{pH}$ and blood gases $\left(\mathrm{pCO}_{2}, \mathrm{pO}_{2}\right)$ for intravascular continuous monitoring with an amperometric enzyme oxygen electrode (Clark et al., 1962). In 1969, another new kind of biosensor, the potentiometric biosensor, 
was invented. This biosensor could detect urea on an ammonia electrode. From 1972 to 1973, the first commercial biosensor, a glucose biosensor, was successfully made by Yellow Spring Instrument (YSI) Company. Stimulated by this commercial success, fundamental studies of biosensors were performed vigorously in 1980s. During these years, not only electrochemical sensors, but also optical sensors were developed. For example, the first fiber optic $\mathrm{pH}$ sensor was developed in 1980; the first fiber opticalbased biosensor for glucose appeared in 1982, and the first surface plasmon resonance (SPR) immunosensor was reported in 1983. Unlike electrochemical sensors, optical sensors have their own advantages. Satisfactory results were obtained by normalizing the optical signal of the analyte to a second reference wavelength, which involved evaluating relative intensity changes. After the 1990s, there has been even more emphasis on the commercialization of biosensors.

Table 1.1. Major landmarks in biosensor development (from Newman et al., 2001).

\begin{tabular}{|c|c|}
\hline Date & Event \\
\hline 1962 & $\begin{array}{l}\text { First description of biosensor: an amperometric enzyme electrode for } \\
\text { glucose }\end{array}$ \\
\hline 1969 & $\begin{array}{l}\text { First potentiometric biosensor: urease immobilised on an ammonia electrode } \\
\text { to detect urea }\end{array}$ \\
\hline $1972-1973$ & First commercial biosensor: Yellow Spring Instruments glucose biosensor \\
\hline 1975 & First microbe-based biosensor; First immunosensor \\
\hline 1980 & First fiber optical $\mathrm{pH}$ sensor for in vivo blood gases \\
\hline 1982 & First fiber optical-based biosensor for glucose \\
\hline 1983 & First surface plasmon resonance (SPR) immunosensor \\
\hline 1984 & First mediated amperometric biosensor \\
\hline 1987 & Launch of the MediSense ExacTech blood glucose biosensor \\
\hline 1990 & Launch of the Pharmacia BIAcore SPR-based biosensor \\
\hline 1998 & Launch of LifeScan FastTake blood glucose biosensor \\
\hline 2004 & SPARROW biosensor proposed (Lloyd et al., 2004) \\
\hline
\end{tabular}


Although biosensors have already become a mature technology in some areas, this maturity is only limited to a small number of applications where market size justified significant financial investment (Newman et al., 2001). A great deal of researches and developments are required in the future for biosensors. In brief, the developments of biosensors can be divided into three generations. The first generation of biosensors is before the 1980s. At that time, both biological components and transducers were very limited. The research and application of biosensors were also very small. In comparison to the first generation, the second generation of biosensors typically used antibodies and proteins instead of enzymes as their biological components, and these have more choices for transducer types. A typical product of this generation was the Pharmacia BIAcore SPR-based biosensor, which was launched to market in 1990. Besides medical applications, many new possible applications of biosensors were found. The third generation of biosensors currently being developed is portable, automatic, and features real-time measuring capabilities.

\section{1-2 Biosensor configurations}

There are numerous literatures discussed biosensor configuration such as enzymebased fiber biosensor (Thompson, et al., 1993), enzyme electrode biosensor (Sampath, et al., 1997), biosensor based on Piezoelectric Crystal (Kumar, 2000), etc. Since 1962, the year of the first biosensor, a great number of combinations have been proposed and demonstrated. However, very few of them have been commercially successful because of a variety of reasons such as fabrication problems, low sensitivity problems, etc. Actually, in simplest terms, only two components are needed for a very basic biosensor: (1) a receptor, which is a 'molecule or a polymeric structure in or on a cell which specifically recognizes and binds to a compound acting as a molecular messenger' (Nagel, et al., 1992); and (2) a transducer, which is used to 'sense the binding event produced by the bioreceptor and analyte, and in turn to generate an electrical or optical signal that can be amplified and measured' (Patton, 1989). When a biosensor works, the receptor will produce a discrete or continuous biosignal. Usually, this signal corresponds to specific biochemical reactions between the receptor and binding events. Then, the transducer will sense this biosignal and convert it to another signal which could be recognized by the 
detection device. Finally, information of the sample will be known by analyzing the output signal. Figure 1.1 shows the principle of the operation of a biosensor.

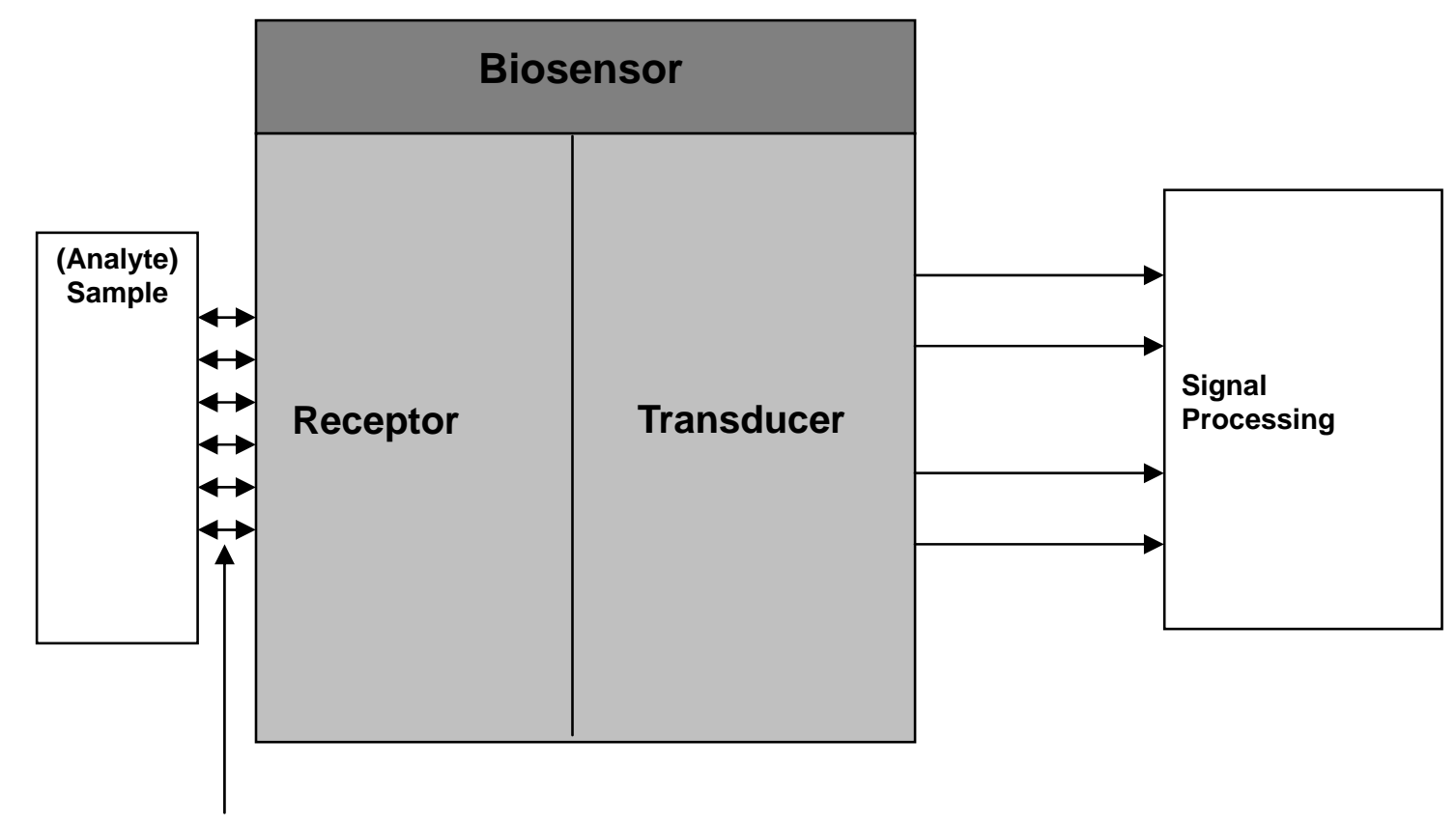

Specific biochemical reaction

Fig 1.1. The principle of the operation of a biosensor.

Based on different types of transducers and receptors, numerous biosensors have been developed and some of them are successfully commercialized. In the following sections, several typical biosensors are introduced.

\section{1-2-1 Amperometric biosensors and potentiometric biosensors}

Amperometric biosensors are the earliest biosensors. The purpose of this kind of biosensors is to detect the concentration of target solution (i.e. glucose concentration in blood) by monitoring the change of current. When the analyte flow through the biomaterial coated electrode, there will be an oxidation or reduction of such biomaterial at electrode surface. Then, a current proportional to oxygen concentration is produced by redox activities. Equation 1.1 shows the common redox cross reaction catalyzed by enzyme (Kissinger, 2006).

$$
R_{A}+O_{B} \stackrel{e n z}{\rightarrow} R_{B}+O_{A}
$$


where $R_{A}$ is analyte and is oxidized by $O_{B} . R_{B}$, which is proportional to current, indicates the concentration of analyte $R_{A}$. For amperometric biosensors, the spices could be urea, tissue, glucose, sucrose, etc. There are three distinct configurations for this kind of biosensors: Single use; Intermittent use; Continuous use. Table 1.2 shows their features.

Table 1.2 Features for different amperometric biosensors configurations (Kissinger, 2006)

\begin{tabular}{|l|c|c|c|l|}
\hline & Precision & Accuracy & Calibration & Cost \\
\hline Single use & Poor & Poor & No & High \\
\hline Intermittent use & Good & Good & Easy & Moderate \\
\hline Continuous use & Good & Poor & No & Low \\
\hline
\end{tabular}

Plenty of receptors for amperometric biosensors corresponding to different detection species have been widely reported in numbers of publications. The limit of detection (LOD) for each combination shows big difference. In a recent literature (Dzyadevych, et al., 2006), the LOD of a Resydrol polymer coated amperometric biosensor was reported to be $3.5(\% \mathrm{v} / \mathrm{v})$ (the volume of the ethanol is $3.5 \%$ of the total volume of the solution). This LOD is excellent for detecting ethanol in target solution.

The same as amperometric biosensors, potentiometric biosensors use electrode as the component of transducer. However, this kind of biosensors monitors the potential change instead of current. They use ion-selective electrodes to sense the biological reaction and covert biosignal into an electrical signal. Figure 1.2 shows one of the simplest structures of this kind of biosensors. It includes an immobilised enzyme membrane and the probe from a $\mathrm{pH}$-meter. The catalysed reaction will generate or absorbs hydrogen ions. That causes a change in $\mathrm{pH}$ which can be measured from the $\mathrm{pH}$ meters. Correspondly, the electrical potential is changed. This change could be detected by detector and used to determine the specific biomaterial in target solution. 


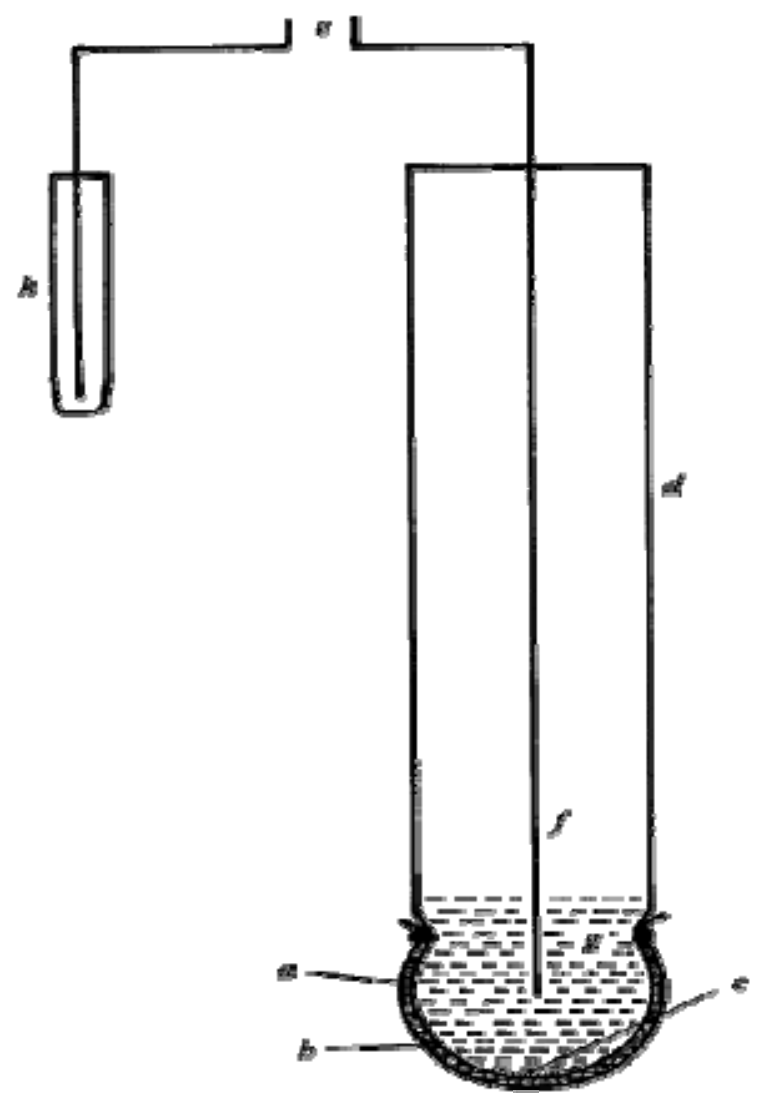

Figure 1.2 A simple potentiometric biosensor. (a) A semi-permeable membrane (b) Biocatalyst (c) The active glass membrane (d) A pH probe (e) The electrical potential (f) Internal Ag/AgCl electrode (g) Dilute $\mathrm{HCl}$ (h) An external reference electrode. (London south bank university)

This kind of biosensors also has high sensitivity. A recent literature reported that an enzyme potentiometric biosensor had LOD of $52 \mu \mathrm{g} / \mathrm{mL}$ when detect the urea concentration (Sahney, et al., 2006).

\section{1-2-2 Optical biosensors}

Optical biosensors detect the biological information by monitoring the change of optical characteristic such as intensity, wavelength, phase, polarization and time modulation. It consists of an optical transducer and a bioreceptor. Optical transducers, such as fiber, surface plasmon resonance device, optrodes and optical waveguides, are 
widely reported and show good promise.

\section{1-2-2-1 Optical fiber biosensors}

The optic fiber biosensors have been developed over the past 20 years from a single channel laboratory breadboard into a portable, automated multiple channels sensor (Anderson, et al., 2000). Although, the components of optical fiber biosensors have evolved significantly, the principle of them has remained the same. Biolayer (or bioreceptor, i.e. antibody) is immobilized onto the surface of an optical fiber. When target solution flows over the fiber probes, immobilized bioreceptor will capture those specific molecules in target solution and form binding events which are called fluorescent complex here. By evanescently exciting surface-bound fluorophores with a diode laser, these biosensors can monitor these complex formations. The optical probe will sense the emitted fluorescence and transmit them to the photodiode detector through optical fiber. On the fiber probe surface, the excitation intensity and efficiency of fluorescence recovery attenuates exponentially with distance. Thus, this system is highly discriminatory for the surface bound fluorophores (Anderson, et al., 2000). Figure 1.3 shows a simple optical fiber structure.

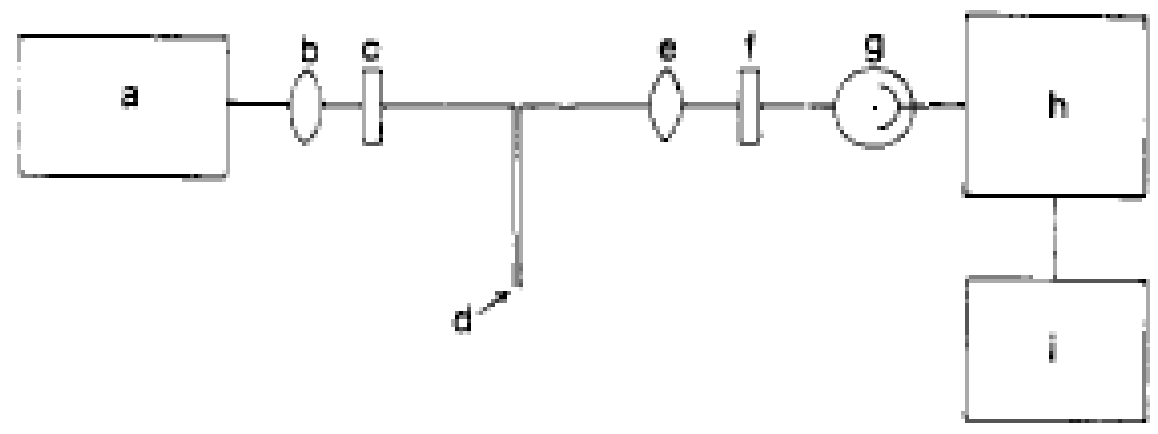

Fig. 1.3. A simple optical fiber biosensor arrangement: (a)Light source; (b) Focusing lens; (c) Neutral density filter; (d) Biosensing tip; (e) Collimating lens; (f) Narrowband-pass filter: (g) Photomultiplier tube; (h) Photomultiplier readout (i) Strip chart recorder. (Anal. Chem., 1985)

Also, the LOD of optical fiber biosensors varies a lot for different bioreceptor and different analyte. A recent literature about a FRET-based optical fiber biosensor for rapid 
detection of Salmonella typhimurium reports that the LOD of such device approaches $0.033 \mathrm{mg} / \mathrm{mL}$ (Grant, et al., 2006).

\section{1-2-2-2 Surface plasmon resonance (SPR) biosensors}

According to the online Wikipedia encyclopedia, the SPR is defined as the excitation of surface plasmons by light is denoted as a SPR for planar surfaces or localized surface plasmon resonance (LSPR) for nanometer-sized metallic structures. Generally, this technique is used to measure the binding interactions of very small amounts of analyte. The binding events formed on membrane (biolayer) result in changes in membrane surface plasmon resonance. Figure 1.4 shows the structure of this kind of biosensors.

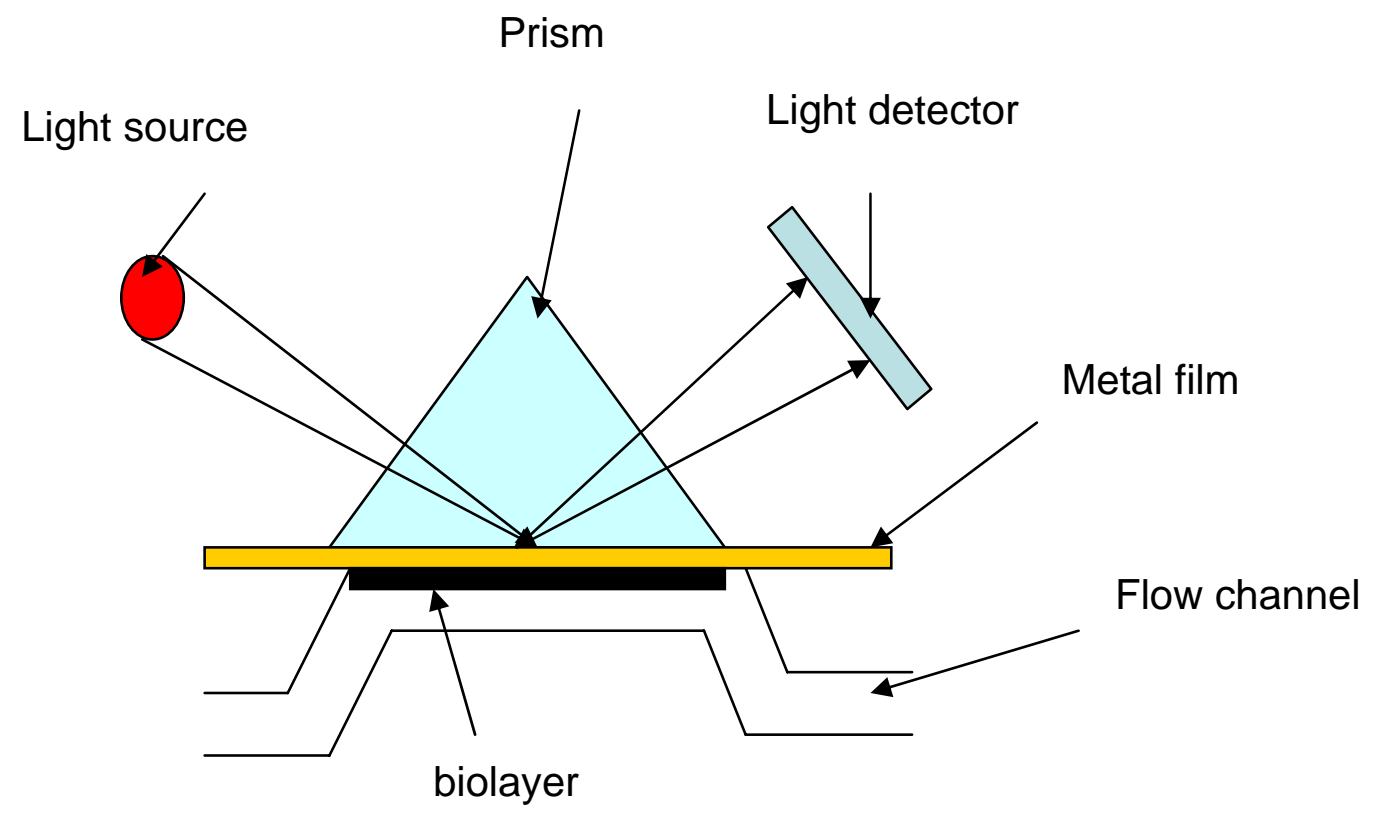

Fig. 1.4. Schematic of SPR biosensor

When such a biosensor works, a light is reflected from thin metal films. A fraction of this light energy can interact with the delocalised electrons in the metal film (plasmon) thus reducing the reflected light intensity at a certain incident angle. This certain angle of incidence is determined by a number of factors such as the refractive index of biolayer. The change of biolayer refractive index results in changes of this certain incident angle. 
Thus, by monitoring this angle change we can detect the analyte in flow channel (See Fig. $1.5)$.

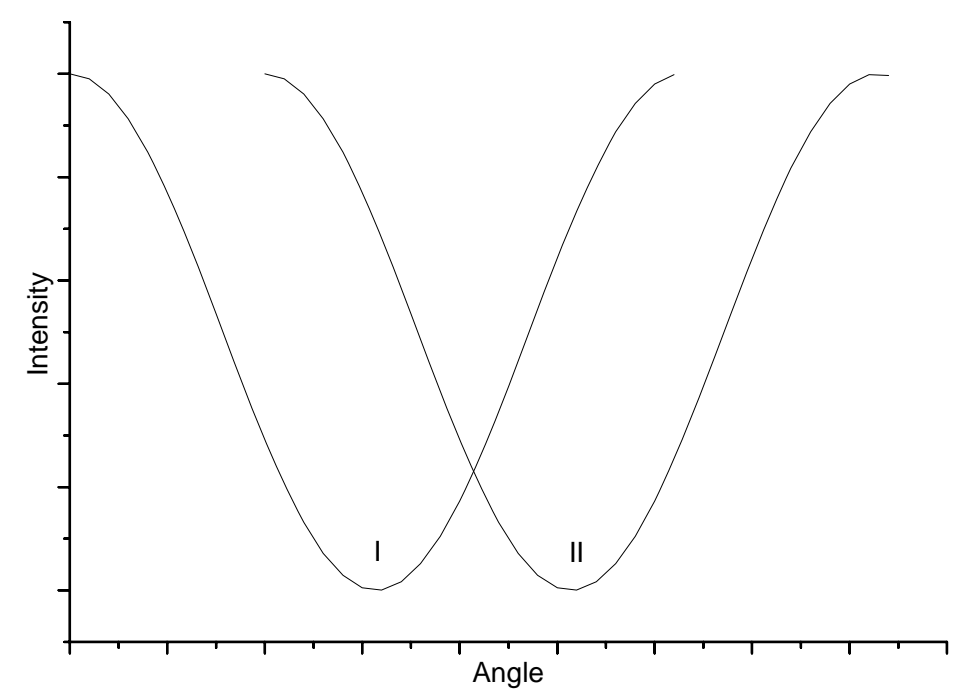

Fig. 1.5. Changes of intensity at certain incident angle. I: Without binding events. II: With binding events.

A SPR biosensor is an important member in biosensors family. The LOD of a commercial SPR biosensor for detection of TNT could approach $0.095 \mathrm{ng} / \mathrm{mL}$ (Shankaran, et al., 2006).

Besides these biosensors which we have talked, there are also many other types of biosensors are reported in literature such as thermometric biosensors, quartz crystal biosensors, etc. They detect the binding events by monitoring changes of temperature and mass, respectively. For each kinds of biosensors, many different bioreceptors have been tested and used, including polyclonal and monoclonal antibodies, their Fab (Fragment antigen binding) fragment, DNA and RNA nucleic acid fragments, and membrane bioreceptors. In addition, whole cells or even micro-organisms are used to specifically bind and often react with the target sample of interest. Molecular sensing labels that directly or indirectly produce light, electrochemical, radioactive, and mass-change signals are numerous. Enzymes that are especially popular provide means to amplify signals by catalytically producing thousands of signal events for a single binding event. The use of multiple enzyme systems can also yield extremely sensitive detection limitation (Patton, 
1989).

Table 1.3 lists advantages and disadvantages in three typical types of biological components. There are some combinations of biological components and transducers that work particularly well together (e.g., enzymes combine with electrochemical transducers and antibodies combine with optical transducers). Research is being carried out to develop novel combinations and component types that may provide new applications for biosensors.

Table 1.3. The advantages and disadvantages of three types of biological components (Bio-wise web site).

\begin{tabular}{lll}
\hline & Advantages & Disadvantages \\
\hline Enzymes & $*$ simple to operate & $*$ need to control ambient conditions \\
& $*$ simple design & $*$ limited shelf life \\
& $*$ sensitive to target analyte & $*$ May not be a suitable enzyme to act \\
& $*$ Rapid result & on the sample analyte \\
& $*$ inhibited enzymes can be & $*$ catalytic biosensors may be inhibited \\
& & by used to determine general \\
& & substance in the sample toxicity \\
& & caused by unknown substances \\
\hline Antibodies & $*$ highly sensitive to target & $*$ still an emerging technology so target \\
& analyte & analytes may be limited \\
& $*$ easy to use in the field & specific for target analyte only so \\
& $*$ easy to interpret & \\
& $*$ rapid result & $*$ take longer time to get results \\
Micro- & $*$ sensitive indicators of & \\
organisms & toxicity & \\
& $*$ longer shelf life & \\
& $*$ tolerant of suboptimum & \\
& conditions
\end{tabular}

\section{1-3 Biosensor applications}

Biosensors can be used for a range of applications and for a number of analytes and parameters. Early research and commercialization have been primarily focused on medical and healthcare applications, mainly for glucose detection. This is reflected in the large share of the biosensors market for medical applications. Table 1.4 shows the global biosensors markets by sector in 1996. At that time, the global biosensor market was 
worth more than $\$ 500$ million/year (Bio-wise web site, 2001).

Table 1.4 Global biosensors markets by sector in 1996 (Bio-wise web site, 2001).

\begin{tabular}{lcl}
\multicolumn{1}{c}{ Sector } & Markets (\%) & Main uses \\
\hline Medical glucose & 90 & Self-Monitoring of glucose by diabetics \\
\hline Medical: others & 2 & Monitoring of lactate, urea, etc \\
\hline Environmental & 2 & Mainly BOD (biological Oxygen Demand) indicators \\
\hline Others & 6 & Fermentation monitoring in the food and drink industry \\
\hline
\end{tabular}

Over the past 10 years, biosensor research is ongoing and further applications are continually being developed. The versatility of biosensors means that there is an increasingly wide range of applications for biosensors within many different industries (Spichiger-Keller, 1998; Diamond, 1998; Ramsay, 1998; Kress-Rogers, 1997). So far, the biosensor global market is worth more than $\$ 6$ billion per year, and expected to be increase to $\$ 8$ billion per year before 2010. However, medical and healthcare clinical applications still play the leading role. A brief review of the clinical, environmental, and process/product control applications are presented next.

\section{1-3-1 Clinical applications}

Most of the on-going biosensor researches, commercial biosensor products, published biosensor papers, and books of biosensors are related to clinical projects. Biosensors are being widely applied to disease detection, as well as healthcare management. In this section, three major clinical applications of biosensors are introduced.

\section{A). Biosensor for personal diabetes management}

Diabetes is a disease in which the body is no longer able to regulate the level of glucose in the blood. Currently, it is estimated that there are 8 million diabetics and an additional 8 million diabetics who have not yet been diagnosed in the United States. Every year, the amount of money spent on treating diabetes is comparable to the amounts spent on heart disease and cancer treatments. To lower diabetes treatment costs, more at- 
home testing is needed. The glucose biosensor has been widely used, and has become the most financially successful sensor among all types of biosensor. In the home healthcare area, the glucose biosensor can be considered as the best model for future biosensor development (Ramsay, 1998).

B) Noninvasive biosensors in clinical analysis

Metabolite measurements in media other than blood are becoming increasingly important in recent years. Those patients who have problems providing blood samples benefit from such measurements. The advantage of easy sample collection allows samples to be collected more frequently with much less stress on the patient. Biosensors do this work very well. They can provide an easy way to get body information by measuring saliva, sweat, and transbuccal mucosa samples (Ramsay, 1998). Currently, there are several kinds of noninvasive biosensors developed to detect analytes such as alcohol, glucose, and lactate.

\section{C) Biosensors for cancer}

According to the American Cancer Society, early detection for many types of cancer (lung, breast, prostate, etc.) will significantly improve the survival chances of patients. If all Americans underwent early detection testing, according to the ACS recommendations, the 5-year relative survival rate for people with these cancers would increase to around $95 \%$. The general methods for early detection only rely on regular body examination. However, the instruments don't always detect miniscule changes in cells. Sometimes even patients known to have been exposed to carcinogens may not show any signs of cancer until it is too late (Energy Science News web site). So, the constant challenge is in producing cost-effective biosensors for cancer detection that show increasing levels of rapid, convenience, sensitivity, and stability. For this purpose, some new biosensors which can aid pre-cancer diagnoses have been developed. With these biosensors, scientists can keep track of DNA adducts formed in human cells and address those cancer cells. Also, biosensor technology is being used to characterize the function of therapeutic antibodies. By using some of these biosensors, diagnostic testing is carried out near or at the bedside and provides results that impact patient care almost 
immediately. (Singapore's Institute Of Bioengineering And Nanotechnology web site)

\section{1-3-2 Environmental applications}

Traditional instrumental analysis used in monitoring and detecting environmental pollution is usually costly and time consuming. For environment protection, the analysis methods must be simple, fast, precise, and reasonable. These requirements can be realized with biosensors (Ramsay, 1998). Such sensors, which consist of enzymes, micro-organisms, antibody, organ-cells, or cells and tissue of animals and plants in intimate contact with the transducer, convert the biochemical reaction to a quantifiable electrical signal. The aim of this combination is the sensitive determination of a large spectrum of substances in various areas, especially pollution control. Biosensors allow

rapid measurement over a wide concentration range without pretreatment, even in colored, turbid samples. Since the 1990s, biosensors of this type have been used successfully for measuring BOD (Biological Oxygen Demand), detecting specific micro-organisms in the drinking water, food, and pharmaceutical products (Ramsay, 1998).

Another important application of biosensors is to detect toxic vapors. General testing is often carried out in a laboratory under strictly controlled conditions. The whole process can take several hours to several days because of the time involved in transporting samples and performing the tests. Routine monitoring in this way is, therefore, usually expensive. There is a need for a quick, easy, and cheaper alternative or a screening method to reduce the number of samples being sent for analysis. Currently, a few commercially available biosensors satisfy this need. Further developments are focusing on ways to deliver cheaper, disposable testing methods that will satisfy the monitoring needs of a wide range of industries (Kress-Rogers, 1997).

In recent years, biosensors have also been used for homeland security systems. For the detection of bioterrorist agents, three types of sensing system have to be employed: 1), generic detector for early warning; 2), biosensors to screen rapidly and make a presumptive identification; and 3), confirmatory identification systems. Usually, biosensors address the second need (Shriver et al., 2005). They provide a cheap, rapid, and automated way to screen samples and identify the biological threat agent. The resonant integrated optical waveguide biosensor, which was proposed (Lloyd et al., 2004) 
two years before, is one of these kinds of biosensors.

\section{1-3-3 Process/product control}

Modern industries are required to monitor and control their manufacturing processes. To indicate how good the process is performing, the 'Real-time' information of product formation is needed. Typically, this type of monitoring involves the detection of minute concentrations of substances. Biosensors offer an effective and rapid means of process and product control in applications including (Terry, 2005):

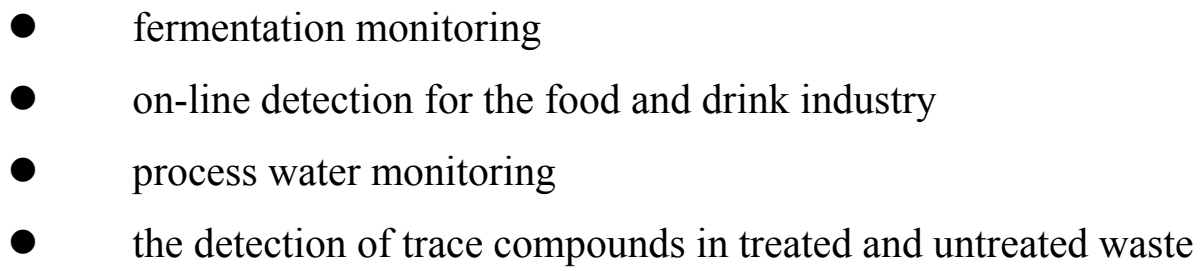

\section{1-4 Resonant integrated optical waveguide biosensor}

The earliest integrated optical device is the photophone invented by Alexander Graham Bell in 1880. The photophone was a device that varied the intensity of sunlight incident upon it in response to the amplitude of speech vibration. A receiver could then be used to reconvert the light variations into an electrical signal, and then back to sound. Though the photophone itself was impractical due to the rapid decrease in intensity with propagation distance, the concept of optical communication that it demonstrated is in many ways responsible for the development of the field of integrated optics (Lee, 1986). Early research on integrated optical devices was focused on optical communication, but in later years, it encompassed many topics such as optical waveguiding, switching, modulation, filtering, interferometry, signal processing, waveguide coupling, optical generation, detection, optical sensing, and so on. Based on past research of integrated optical devices and biosensors, numerous integrated biosensor designs have been reported in the literature capable of direct optical detection in the laboratory (Gilbert et al., 1996; Shriver et al., 2005; Huang et al., 2004; Yuk et al., 2005; Patton, 1989). However, these new device designs are often inconsistent with migration to field-use in a dockable handheld unit since chip design can quickly increase fabrication and optical alignment 
complexity, which in turn inhibits rapid chip docking and interchange (Lloyd et al, 2004). In addition, most of these designs didn't achieve satisfactory sensitivity and stability. To solve these problems, a new kind of biosensor - a stack planar affinity regulated resonant optical waveguide (SPARROW) biosensor was proposed (Lloyd et al., 2004). Based on resonant integrated coupled optical waveguides, this biosensor greatly reduces fabrication and optical alignment complexity. Also, it takes advantage of the optical properties of planar optical waveguide structures and can provide a biosensor device with high operational stability and sensitivity.

\section{1-4-1 Structure and Fabrication}

Figure 1.3 shows the structure of a SPARROW biosensor. The integrated optical chip architecture is designed to take advantage of state-of-the-art ion-beam-assisted thinfilm deposition techniques to reduce fabrication and optical alignment complexity and provide a biosensor device with high operational stability and sensitivity that is amenable to field applications. Specific molecules in a target bioagent solution can be detected by monitoring and analyzing changes in the output power.

The substrate shown in Figure 1.6 is flat Borofloat glass. At a wavelength of 633 $\mathrm{nm}$ (from a He-Ne laser), the refractive index of this special glass is 1.4701. This material was selected for its high chemical and water resistance, low thermal expansion, and high melting point. Over a long period of time at $\mathrm{T}>100^{\circ} \mathrm{C}$, Borofloat glass exceeds the chemical resistance of most materials. Moreover, as a production glass, it is far less expensive than fused silica or quartz. Annealed $\mathrm{Al}_{2} \mathrm{O}_{3}$ was selected to be the waveguide material. This was deposited from an E-Beam evaporation system layer-by-layer during which the thickness can be precisely controlled (Nightingale et al., submitted, 2006; Baumann et al., 1990). At $633 \mathrm{~nm}$, this waveguide material exhibits very low transmission loss. Several minutes of annealing at temperatures between $450^{\circ} \mathrm{C}$ to $650^{\circ} \mathrm{C}$ 


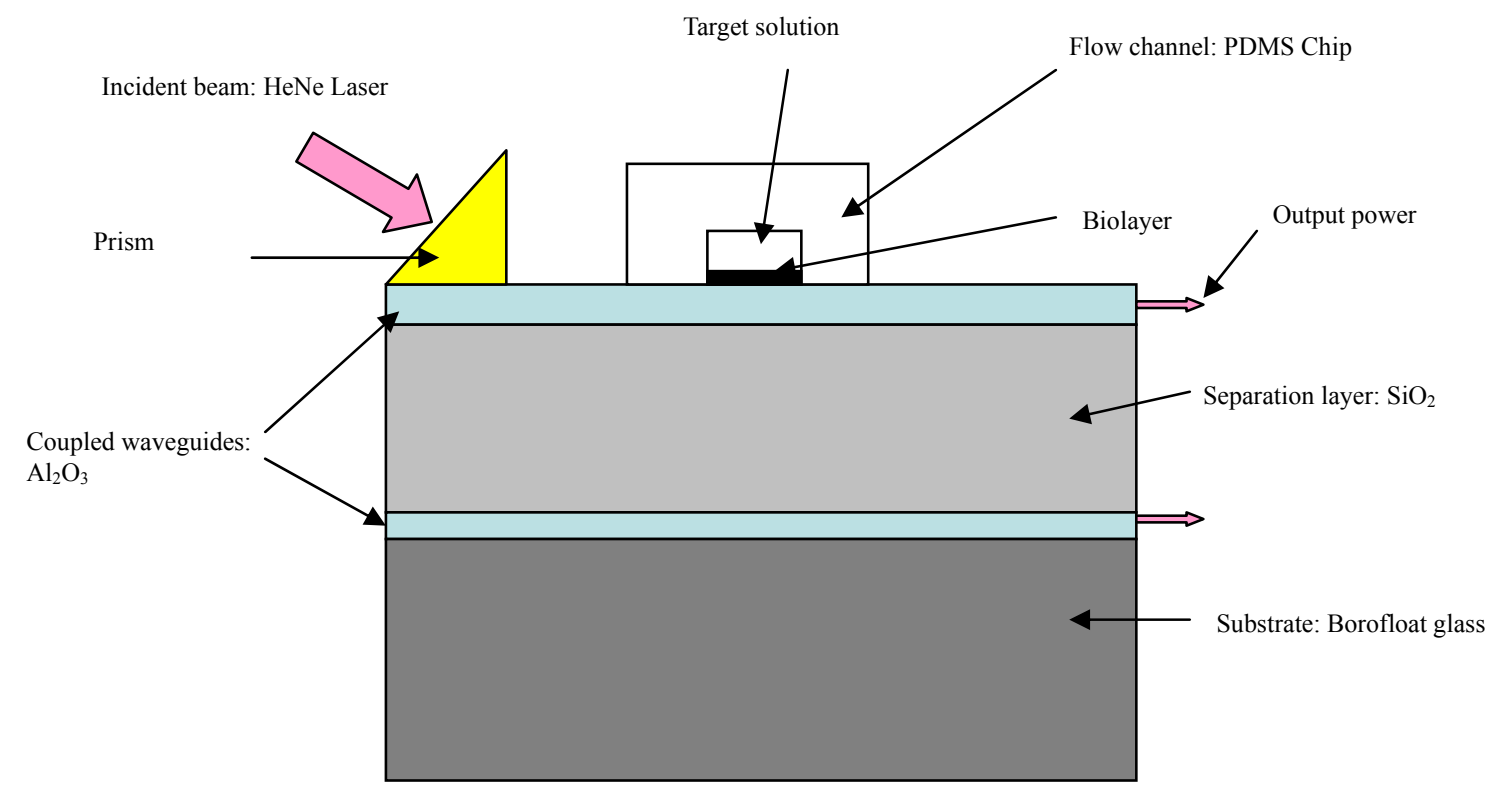

Fig 1.6. Schematic structure of a resonant integrated optical waveguide biosensor.

help reduce surface roughness which leads to a remarkable reduction of transmission loss. Previous tests have proved that transmission losses in aluminum oxide film were reduced from over $10 \mathrm{~dB} / \mathrm{cm}$ to $1 \mathrm{~dB} / \mathrm{cm}$ after annealing (Lloyd et al., 2004). Annealing also increased the stability and density of the films. In addition, the refractive index of $\mathrm{Al}_{2} \mathrm{O}_{3}$ waveguide will slightly change after annealing.

The initial $\mathrm{Al}_{2} \mathrm{O}_{3}$ layer deposited on the Borofloat substrate (1/16 inch in thickness) forms the bottom (or buried) waveguide of the device. Its thickness is approximate 0.144 $\mu \mathrm{m}$. The middle layer of the structure shown in Figure 1.3 is composed of silicon dioxide $\left(\mathrm{SiO}_{2}\right)$, which provides separation (approximately $\left.1.1 \mu \mathrm{m}\right)$ of the two waveguides. This material has an index of refraction of 1.453 post-deposition and 1.457 post-anneal (Lloyd et al 2004). The third layer forms the top $\mathrm{Al}_{2} \mathrm{O}_{3}$ waveguide of the device and is deposited with the aid of a shadow mask. The shadow mask causes the upper guide to gradually drop its thickness below the modal cutoff. Finally, after sample cleaning and preparation, a polydimethylsiloxane (PDMS) layer is lithographically patterned over the top waveguide to define analyte well regions of different interaction lengths and provide a region for flow-cell attachment (Lloyd et al, 2004). The two waveguide layers form the directional coupler, which is part of the transducer. The biolayer can be designed to 
employ binding of a monoclonal or polyclonal antibody, or can be adapted for a specific protein or DNA/RNA probe. In either case, the target binding changes the effective index of the upper guide and results in power transfer modulation. Given this universality of the transduction, the device has great general applicability and can be tailored to the differentiation of many different organisms or analytes by simply changing the array of proteins presented on the surface. The device is broadly applicable to the detection of bioagents in different types of water, ranging from seawater to tap water, and can also be applied to clinical uses and detection of explosives.

\section{1-4-2 Applications of SPARROW biosensor}

Theoretically, the resonant integrated optical waveguide biosensor can detect any biologically relevant information in the nanoscale range because in previous tests (Lloyd et al., 2004) this device could readily detect a surface loading of 0.2 nanograms $/ \mathrm{mm}^{2}$ with an optimized limit of 0.1 picograms $/ \mathrm{mm}^{2}$. Since it is ultrasensitive, it can be used to detect diseased cells as well as biological agents. Therefore, this device is especially important for homeland security and medical applications such as cancer research. These are described further in the following sections.

\section{A). Homeland security}

In recent years, the war against terrorism has required more emphasis be placed on homeland security. Numerous sensor technologies in this area have been developed. A portable biosensor system for rapid direct detection and identification of hazardous biological agents is needed. The SPARROW biosensor device provides such an effective way to protect and response from sophisticated biowarfare agents to simple biocontamination of food and water supplies. It uses evanescent waves which interact to interrogate specially engineered biolayers incorporated with high affinity molecular probes, such as antibodies, to selectively capture targeted bio-agents (Lloyd et al., 2004; Shriver et al., 2005).

\section{B). Cancer research}

As described in section 1-3-1, early detection will improve a patient's chances of 
recovery, and much work is currently being done to improve cancer diagnostics. Few conventional biosensors have sufficient sensitivities for the detection of genetic material at subpicomolar levels. However, the SPARROW biosensor, which uses evanescent wave technology, is much more sensitive than these other biosensors. This biosensor is coated with a nanofilm (we call biolayer, total thickness around $10 \mathrm{~nm}$ ) which can capture cells on its surface. When loading the sample solution on top of a SPARROW

biosensor, these samples will hybridize with the biomaterials of the biolayer. The intensity of the optical signal correlates directly to the amount of diseased cells. In fact, this biosensor is expected to be very responsive, and hopefully it can recognize some cancer susceptibility genes in messenger RNA extracted from human body tissues without involving a polymerase chain reaction (PCR) step, which is typically used to amplify the gene expression. Besides being able to detect disease-associated DNA/RNA at a rapid rate, the device is also cost-effective. In addition, the biosensor is highly compact and mobile, and is compatible with advanced semiconductor technology. It is expected that with the development of a reliable molecular diagnostic device, some cancers can be detected at a very early stage.

\section{1-5 Motivation and objectives}

As described in the previous sections, the SPARROW biosensor has high sensitivity and stability and can be widely used in many important areas. However, the quality of such device can be easily affected by slight changes in many factors such as film thickness, biolayer coverage, interaction length of waveguide, etc. With fabrication limitations, it is difficult for people to distinguish the dominant factor by only experimental methods. In order to comprehensively understand and optimize this device, an analytic predictive model is needed. Understanding biolayer design and establishing appropriate mathematical models are important aspects of this dissertation work. I developed a numerical method that not only enables increased understanding of this special biosensor but also guides the device design and optimization. In my dissertation, this project is divided into three parts: (1) ideal SPARROW biosensor modeling, (2) biolayer design, (3) non-ideal SPARROW biosensor modeling. The first part focuses on description of the structure and complex optical behavior in ideal coupled waveguides 
and their mathematical properties. The focus of the second part is introducing biolayer construction which offers many parameters in biosensor modeling. The third part describes an improved mathematical model to match the real device. The influence of experimental variables such as roughness and coverage of binding events are numerically evaluated.

My work is organized as follows. Chapter 2 introduces the theoretical principle of SPARROW biosensor. This includes applications of ray optics and wave optics in slab waveguides, effective refractive index, evanescent wave technique and coupling mode theory. In Chapter 3, a finite difference beam propagation method (FD-BPM) is applied to perform computer simulations of device structure. Results are compared with preliminary experimental works on the actual sensor structure. In addition, an analytical model of this ideal SPARROW biosensor structure is described and the results are compared with computer simulations. Chapter 4 focuses on biolayer construction and experimental techniques such as ellipsometry, contact angle measurement, atomic force microscopy (AFM) and fourier-transform infrared (FTIR) spectroscopy to detect the organic film structure. In Chapter 5, an analytical model for a non-ideal SPARROW biosensor (imperfect surface, non-uniform refractive index, random binding events) is discussed. Based on this model, the SPARROW biosensor behavior can be predicted and optimized. Chapter 6 is my dissertation summary and conclusion. 


\section{Chapter 2}

\section{Principles of SPARROW biosensor waveguides}

A SPARROW biosensor includes two parts - a transducer and a biolayer. The biolayer is used to sense specific biological binding events and to generate a signal. A directional coupler, composed by two slab dielectric waveguides, is used as a transducer to transmit the optical signal. To understand how the SPARROW biosensor works, it is necessary to first consider the optical processing which occurs in the slab dielectric waveguide. This chapter provides a basic approach to understand the beam behavior in coupled waveguides. Once the beam transmission properties in coupled waveguides are established, further mathematical modeling of the SPARROW biosensor can be performed.

\section{2-1 Basic theory of a slab dielectric waveguide}

A waveguide is a structure that allows optical confinement by making use of multiple total internal reflections from two or more interfaces (Snyder, 1988). Usually, this structure involves three media: a cladding layer, a thin film, and a substrate. These layer interfaces are parallel to each other unless otherwise specified. Generally, to guarantee efficient light transmission in the thin film, this waveguide layer should have an index of refraction larger than the either cladding or the substrate layers. In the simplest structure of waveguide, both cladding and substrate layers are air.

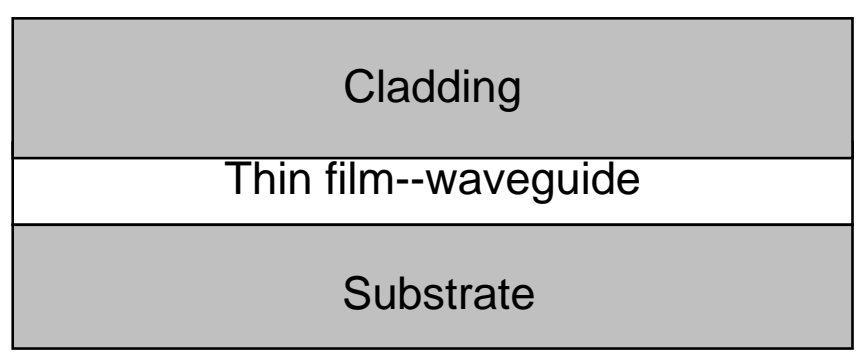

Fig 2.1. Schematic of waveguide structure. 
A slab dielectric waveguide is basically a dielectric medium of one refractive index imbedded in a medium of lower refractive index such that the medium with the higher refractive index acts as a light trap or guide (Marcuse, 1991). This dielectric medium has a rectangular cross section. It can be used in various applications such as guiding energy, transmitting light, etc. Analysis of the light propagation in such a waveguide will provide significant insight into the behavior of the waveguide-based transducer.

\section{2-1-1 Basic electromagnetic approach}

Light has a dual nature-it exhibits particle-like behavior during emission and absorption processes, as well as wave-like behavior during propagation. In a slab electric waveguide, light is usually treated as an electromagnetic wave. The passage of light through a slab dielectric waveguide can be well described by the wave equation. To obtain the wave equation in such a waveguide, we first take the curl of Maxwell's equations (Faraday's Law and Ampere's Law). The results are given in Eq. 2-1.

$$
\begin{aligned}
& \nabla \times(\nabla \times \vec{E}(r, t))=\nabla \times\left(-\frac{\partial}{\partial t} \vec{B}(r, t)\right) \\
& \nabla \times(\nabla \times \vec{B}(r, t))=\nabla \times\left(\mu \varepsilon \frac{\partial}{\partial t} \vec{E}(r, t)\right)
\end{aligned}
$$

Assuming the electric and magnetic fields vary at a sinusoidal frequency $\omega$, Maxwell's equations then yield

$$
\begin{aligned}
& \nabla \times \vec{E}(r)=-i \omega \mu \vec{H}(r) \\
& \nabla \times \vec{H}(r)=i \omega \varepsilon \vec{E}(r)
\end{aligned}
$$

which can be combined with Eq. 2-1 to give

$$
\nabla^{2} \vec{E}(r)+\omega^{2} \mu \varepsilon \vec{E}(r)=0
$$

Equation 2-3 is well known as the wave equation. In a rectangular coordinate system, this wave equation represents three equations of identical form, one for each component of $\boldsymbol{E}(\boldsymbol{r})$ (Lee, 1986). 


$$
\begin{aligned}
& \left(\frac{\partial^{2}}{\partial x^{2}}+\frac{\partial^{2}}{\partial y^{2}}+\frac{\partial^{2}}{\partial z^{2}}\right) E_{x}(r)+\omega^{2} \mu \varepsilon_{x} E_{x}(r)=0 \\
& \left(\frac{\partial^{2}}{\partial x^{2}}+\frac{\partial^{2}}{\partial y^{2}}+\frac{\partial^{2}}{\partial z^{2}}\right) E_{y}(r)+\omega^{2} \mu \varepsilon_{y} E_{y}(r)=0 \\
& \left(\frac{\partial^{2}}{\partial x^{2}}+\frac{\partial^{2}}{\partial y^{2}}+\frac{\partial^{2}}{\partial z^{2}}\right) E_{z}(r)+\omega^{2} \mu \varepsilon_{z} E_{z}(r)=0
\end{aligned}
$$

Eq. 2-4 can be expressed in the $\mathrm{x}$ direction as

$$
E_{x}(r)=E_{x 0} \exp \left[-i\left(k_{x} x+k_{y} y+k_{z} z\right)\right]
$$

where $\boldsymbol{E}_{\boldsymbol{x} \boldsymbol{0}}$ is a constant which represents the amplitude of the electric field. Due to the exponential form chosen for $\boldsymbol{E}_{\boldsymbol{x}}(\boldsymbol{r})$, the effect of operating $\partial^{2} / \partial x^{2}$ on $\boldsymbol{E}_{\boldsymbol{x}}(\boldsymbol{r})$ is to yield $-k_{x}^{2} E_{x}(r)$ with analogous results for partials with respect to $\mathrm{y}$ and $\mathrm{z}$. Thus, the differential equations yield

$$
k_{x}^{2}+k_{y}^{2}+k_{z}^{2}=\omega^{2} \mu \varepsilon \equiv k^{2}
$$

Equation 2-6 is referred to as the dispersion relation. Also, we can define a wavevector $\vec{k}$ given by

$$
\vec{k}=\hat{x} k_{x}+\hat{y} k_{y}+\hat{z} k_{z}
$$

Then, $\boldsymbol{E}_{\boldsymbol{x}}(\boldsymbol{r})$ can be represented as

$$
E_{x}(\vec{r})=E_{x 0} \exp (-i \vec{k} \cdot \vec{r})
$$

where the wavevector $\vec{k}$ represents the propagation direction of the electromagnetic radiation. Then, the direction in which the wave is traveling can be determined by specifying $\boldsymbol{k}_{\boldsymbol{x}}, \boldsymbol{k}_{\boldsymbol{y}}, \boldsymbol{k}_{z}$. Equation 2-9 gives the magnitude of $\vec{k}$ as

$$
|\vec{k}| \equiv k=\omega \sqrt{\mu \varepsilon}
$$

The $k$ surface composes a closed sphere which has radius $k$. We can choose any propagation direction lying on this sphere. Radiation of this form given by Eq. 2-5 is called 'a plane wave' (Lee, 1986). Moreover, in a source-free region, Maxwell's equations for plane-wave solutions can be rewritten as 


$$
\begin{aligned}
& \vec{k} \times \vec{E}=\omega \mu \vec{H} \\
& \vec{k} \times \vec{H}=-\omega \mu \vec{E} \\
& \vec{k} \cdot \vec{E}=0 \\
& \vec{k} \cdot \vec{H}=0
\end{aligned}
$$

Thus, if $\vec{k}$ is along the $\mathbf{z}$ direction, multiplying the complex fields by $\exp (i \omega t)$ and just keeping the real part, the time-space variations for the fields $\boldsymbol{E}$ and $\boldsymbol{H}$ are

$$
\begin{aligned}
& \vec{E}(r, t)=\hat{x} E_{x 0} \cos \left(w t-k_{z} z\right) \\
& \vec{H}(r, t)=\hat{y} \frac{E_{\times 0}}{\sqrt{\mu / \varepsilon}} \cos \left(\omega t-k_{z} z\right)
\end{aligned}
$$

Here, $\boldsymbol{E}$ and $\boldsymbol{H}$ are everywhere in phase. $k_{z}$ is the wavevector component in the $\boldsymbol{z}$ direction and depends on the light wavelength and the refractive index of the material.

\section{2-1-2 Light behavior at the slab dielectric waveguide interface}

As described before, a slab dielectric waveguide has three layers. The optical properties of the waveguide often exhibits discontinuous jumps at the interface between each two layers. Analysis of optical phenomenon of such interface will provide the key to understanding light behavior in a slab dielectric waveguide.

\section{2-1-2-1 Boundary conditions between two dielectric interface}

Generally, when we explore electromagnetic waves in a confinement structure, we need to obtain the boundary conditions first. To directly obtain the boundary conditions in a slab dielectric waveguide, we integrate Maxwell's equations instead of using Maxwell's differential equations. The result is given in Eq. 2-12

$$
\begin{aligned}
& \oint_{c} \vec{E} \cdot \hat{l} d l=-\frac{\partial}{\partial t} \oiint_{s} \vec{B} \cdot \hat{n} d S \\
& \oint_{c} \vec{H} \cdot \hat{l} d l=-\frac{\partial}{\partial t} \oiint_{s} \vec{D} \cdot \hat{n} d S
\end{aligned}
$$


As shown in Fig. 2.2, $\hat{l}$ is a unit vector along the surface direction and $\hat{n}$ is the unit vector normal to the differential surface element $\mathrm{d} \boldsymbol{S}$. $\boldsymbol{C}$ is the length of the closed line which bounds the surface $\boldsymbol{S}$. When $\vec{E} \cdot \hat{l}$ and $\vec{H} \cdot \hat{l}$ approach their tangential values $\boldsymbol{E}_{\text {tan }}$ and $\boldsymbol{H}_{\text {tan }}$ at the interface, the right sides of the two expressions in Eq. 2-12 will approach zero. Thus, we can conclude that the tangential components of $\vec{E}$ and $\vec{H}$ must be continuous across the dielectric interface at all points along the boundary. This conclusion is known as the dielectric interface boundary condition (Lee, 1986).

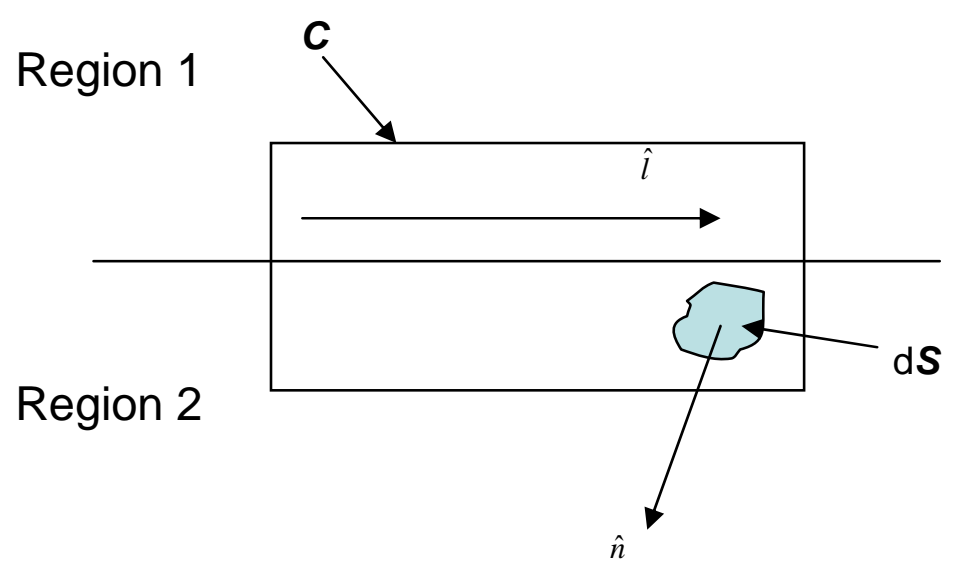

Fig 2.2. Geometry for computation of boundary conditions between regions 1 and 2.

\section{2-1-2-2 Reflection and refraction at the interface}

When light encounters a smooth dielectric interface, its propagation direction could be changed at the boundaries between two dielectric materials. If there is no absorption at this interface and the surface is not perfectly reflecting, then a portion of the beam will be reflected back into Region 1 while the rest of the beam will transmit to Region 2 as shown in Fig. 2.3. The reflecting angle $\theta_{r}$ is equal to the incident angle $\theta_{i}$, while the refractive angle $\theta_{t}$ depends on incident angle and the refractive indices of the two dielectric materials. Mathematically, this refractive angle can be derived from the boundary conditions. When crossing the boundary at $\mathrm{x}=0$, the tangential components of 


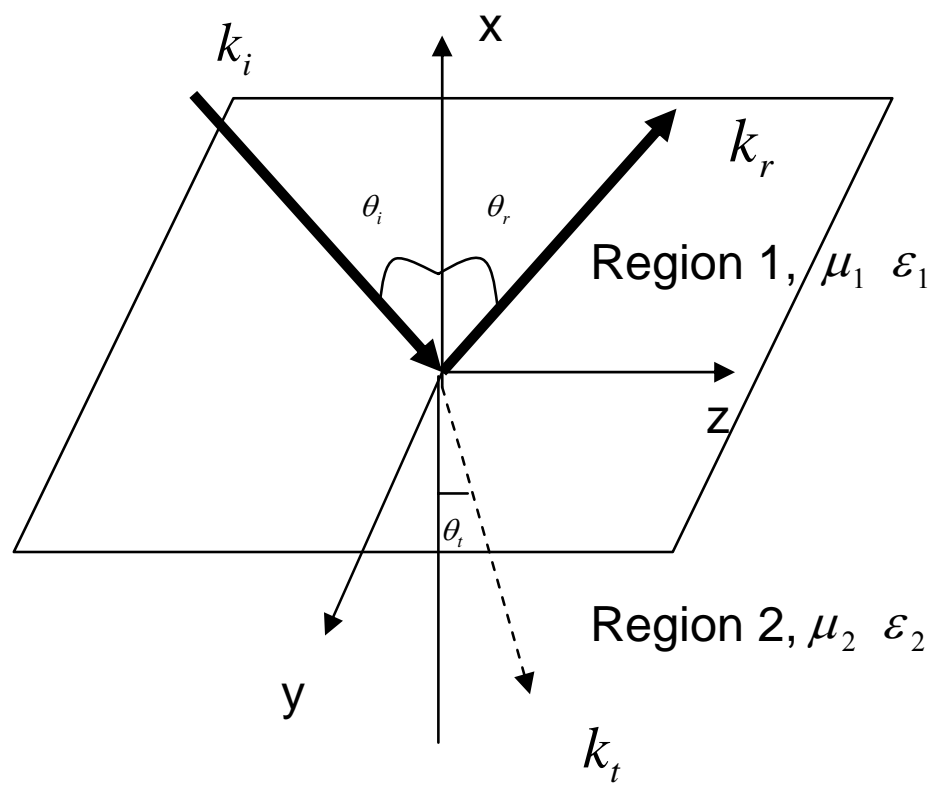

Fig 2.3. Relative orientation between incident, reflected, and transmitted beams. Note that $\mathrm{x}$-direction is chosen to be vertical.

the electric field are continuous. Thus,

$$
\left[E_{i}\left(0^{+}, y, z\right)+E_{r}\left(0^{+}, y, z\right)\right]_{\tan }=\left[E_{t}\left(0^{-}, y, z\right)\right]_{\tan }
$$

For a planar wave, we have $\vec{E}_{r}=E_{0} \operatorname{Exp}(-i \vec{k} \cdot \vec{r}+i \omega t)$. Combining this equation with Eq. 2-11 yields

$$
\begin{aligned}
& k_{i y}=k_{r y}=k_{t y} \equiv k_{y} \\
& k_{i z}=k_{r z}=k_{t z}=k_{z}
\end{aligned}
$$

These relations are known as the phase matching requirements. Physically, they imply that the incident, reflected, and transmitted wave vectors all lie in the same plane. This plane is called the incident plane. In this plane, those angles between the $\mathrm{x}$ axis and the incident, reflected, and transmitted directions are denoted by $\theta_{i}, \theta_{r}, \theta_{t}$, respectively. In terms of these angles, 


$$
\begin{aligned}
& \vec{k}_{i}=-\hat{x} k_{i x}+\hat{z} k_{i z} \\
& \vec{k}_{r}=+\hat{x} k_{r x}+\hat{z} k_{r z} \\
& \vec{k}_{t}=-\hat{x} k_{t x}+\hat{z} k_{t z}
\end{aligned}
$$

where

$$
\begin{array}{ll}
k_{i x}=k_{1} \cos \theta_{i} & k_{i z}=k_{1} \sin \theta_{i} \\
k_{r x}=k_{1} \cos \theta_{r} & k_{r z}=k_{1} \sin \theta_{r} \\
k_{t x}=k_{1} \cos \theta_{t} & k_{t z}=k_{1} \sin \theta_{t}
\end{array}
$$

and

$$
k_{1}=\omega \sqrt{\mu_{1} \varepsilon_{1}} \quad k_{2}=\omega \sqrt{\mu_{2} \varepsilon_{2}}
$$

Eq. 2-15 requires that the tangential or $\mathrm{z}$ component of the incident, reflected and the transmitted wave vectors are the same. Therefore, we have the conditions

$$
\sin \theta_{i}=\sin \theta_{r} \quad \text { and } \quad k_{1} \sin \theta_{i}=k_{2} \sin \theta_{t}
$$

which results in the angle of incidence being equal to the angle of reflection, and the transmitted angle is related to the angle of incidence by the following relation.

$$
\frac{\sin \theta_{i}}{\sin \theta_{t}}=\frac{k_{2}}{k_{1}}=\sqrt{\frac{\mu_{2} \varepsilon_{2}}{\mu_{1} \varepsilon_{1}}}
$$

When both regions have the same permeability, we have

$$
\frac{\sin \theta_{i}}{\sin \theta_{t}}=\sqrt{\frac{\varepsilon_{2}}{\varepsilon_{1}}}=\frac{n_{2}}{n_{1}}
$$

where $\boldsymbol{n}_{1}$ and $\boldsymbol{n}_{2}$ are the indices of refraction of the two regions. This relation is known as Snell's Law. For a certain incident angle, the transmitted angle $\theta_{t}$ will equal $90^{\circ}$. This particular incident angle is called the critical angle $\theta_{c}$. At incident angles greater than $\theta_{c}$, the transmitted direction can not be found because the projection of $k_{t}$ onto the $\hat{z}$ axis is required to exceed its magnitude. From Eq. 2-19, this critical angle can be determined by the following relations.

$$
k_{1} \sin \theta_{c}=k_{2} \quad \text { or } \quad n_{1} \sin \theta_{c}=n_{2}
$$

From these equations, if the incident angle from waveguide to outside is larger than the 
critical angle $\theta_{c}$, there will be no net transmission of light across the surface. All the light energy is limited to within the waveguide (Lee, 1986).

\section{2-1-2-3 TE and TM incident waves}

Since the incident electromagnetic waves are transverse, there are two polarization directions perpendicular or parallel to the surface of the dielectric. If the electromagnetic wave has $\vec{E}$ perpendicular or transverse to the plane of incidence, the wave is called transverse electric or TE (Lee, 1986). Correspondingly, if such a wave has its magnetic field polarized in the plane perpendicular or transverse to the plane of incidence, then we call this wave as transverse magnetic or TM (Lee, 1986). Fig. 2.4 shows the two cases of either TE or TM wave incident.

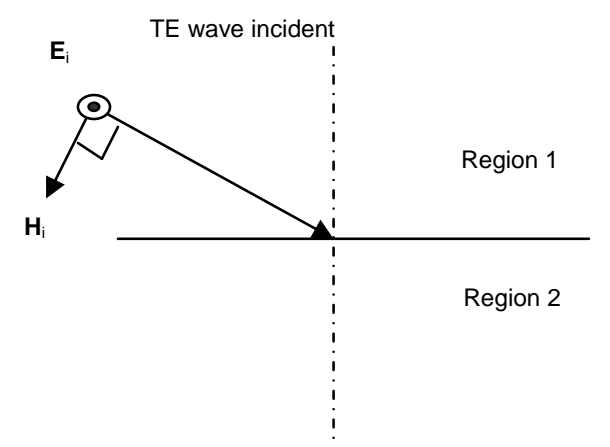

(a)

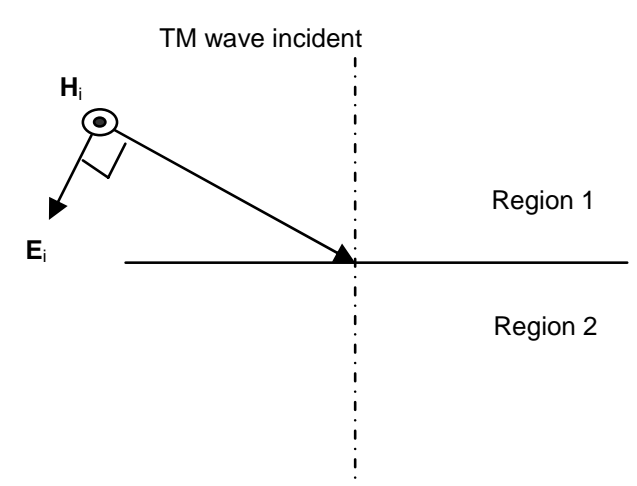

(b)

Fig 2.4 (a) Incident field orientation for TE wave, and (b) incident field orientation for TM wave. 


\section{2-1-3 Light propagation in a dielectric waveguide}

\section{2-1-3-1 Evanescent waves}

An evanescent wave is an electromagnetic wave that decays exponentially with distance. To understand how an evanescent wave is produced in dielectric waveguide,

consider the component of the transmitted wave vector $k_{t}$ in the $x$ direction. The dispersion relation gives

$$
k_{t x}=\sqrt{k_{2}^{2}-k_{z}^{2}}
$$

Due to phase matching,

$$
k_{z}=k_{1} \sin \theta_{i}
$$

which yields,

$$
k_{t x}=\sqrt{k_{2}^{2}-k_{1}^{2} \sin ^{2} \theta_{i}}
$$

We note that for $k_{1} \sin \theta_{i}>k_{2}$ the argument of the square root is negative. The angle for which the argument goes to zero is just the critical angle $\theta_{c}$. So, for $\theta_{i}>\theta_{c}$, $k_{t x}$ becomes purely imaginary

$$
k_{t x}= \pm i \sqrt{k_{1}^{2} \sin ^{2} \theta_{i}-k_{2}^{2}} \equiv \pm i \alpha_{t x}
$$

where $\alpha_{t x}$ is the attenuation factor. Assume the waveguide is homogenous in the $\boldsymbol{y}$ plane so that in the waveguide cross-section (i.e., x-y plane), the time-independent plane wave solution is $\vec{E}_{x}=E_{0} \operatorname{Exp}\left(i k_{t x} x\right)$, so

$$
E_{x}=E_{0} \operatorname{Exp}\left(i\left( \pm i \alpha_{x} x\right)\right)=E_{0} \operatorname{Exp}\left(\mp \alpha_{t x} x\right)
$$

Because the solution should be bounded as $x$ approaches $-\infty$, the only choice is the plus sign, that is

$$
k_{t x}=-i \alpha_{t x}
$$

Thus,

$$
E_{x}=E_{0} \operatorname{Exp}\left(\alpha_{t x} x\right)
$$

So, the field decreases exponentially outside of the waveguide. Fig. 2.5 shows the evanescent wave in a dielectric waveguide of thickness $\boldsymbol{d}$. 


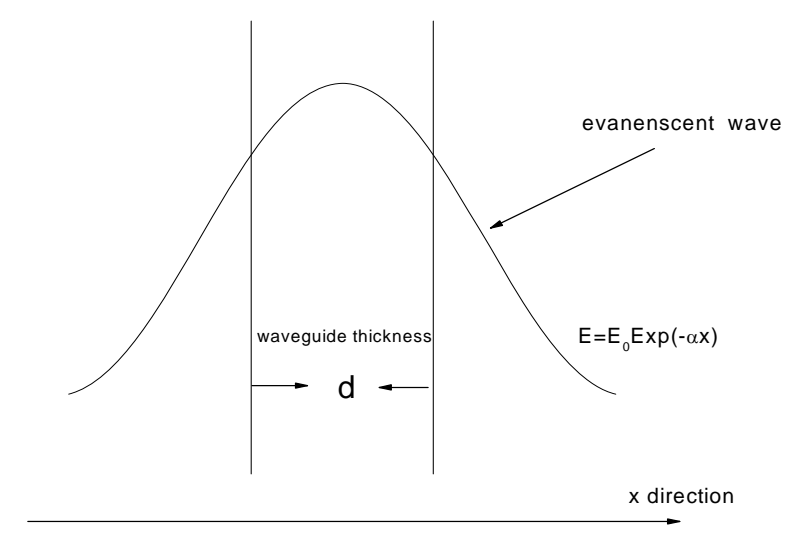

Fig. 2.5. Evanescent wave in a dielectric waveguide.

\section{2-1-3-2 TE and TM modes in symmetric dielectric waveguide}

An electric waveguide mode is defined as a set of electromagnetic fields which maintain their transverse spatial distribution while traveling along a direction of propagation. Let's consider a mode in a symmetric waveguide first. In the symmetric waveguide, the cladding and substrate layers have the same optical properties, meaning their refractive indices are equal. Also, for a slab dielectric waveguide, it is homogenous in the $\boldsymbol{y}$ direction so that all field variations lie in the $\boldsymbol{x} z$ plane and therefore are independent of $\boldsymbol{y}$. Generally, if the propagation direction is along $\boldsymbol{z}$ as shown in Fig. 2.6, the expressions for $\boldsymbol{E}$ and $\boldsymbol{H}$ can be written as:

$$
\left(\begin{array}{c}
E(x, y, z) \\
H(x, y, z)
\end{array}\right)=\left(\begin{array}{c}
E(x, z) \\
H(x, z)
\end{array}\right)=\left(\begin{array}{c}
E(x) \\
H(x)
\end{array}\right) e^{-i k_{z} z}
$$

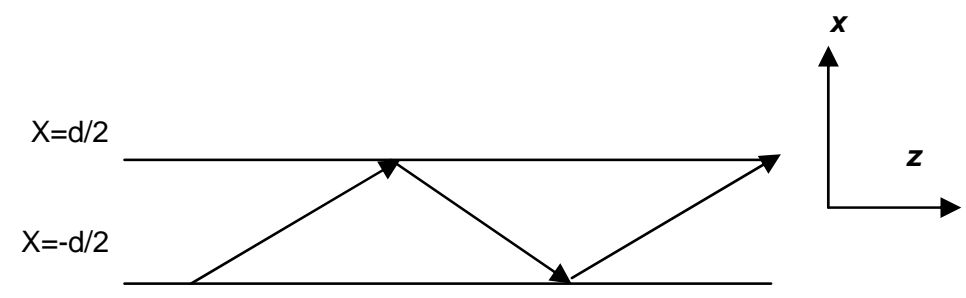

Fig. 2.6. Light propagation in a slab dielectric waveguide of thickness d. 
The TE mode solution can be derived from Eqs. 2-26 and 2-27. In this case, the electric field is polarized along $\boldsymbol{y}$. It has the following form:

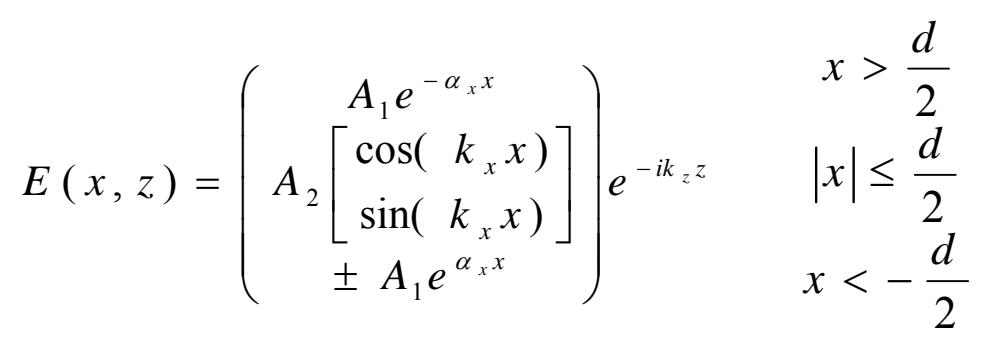

where plus and minus signs correspond to even and odd solutions, and $\boldsymbol{d}$ is the thickness of waveguide. $\mathrm{A}_{1}$ and $\mathrm{A}_{2}$ are amplitude coefficients and the constants $k_{x}$ and $\alpha_{x}$ are obtained from the dispersion relations which are

$$
\begin{aligned}
& k_{x}=\sqrt{\omega^{2} \mu_{2} \varepsilon_{2}-k_{z}^{2}} \\
& \alpha_{x}=\sqrt{k_{z}^{2}-\omega^{2} \mu_{1} \varepsilon_{1}}
\end{aligned}
$$

Also, we can get the magnetic field by taking the curl of Eq. 2-28.

$$
H(x, z)=\frac{-i}{\omega \mu}\left(\begin{array}{c}
\alpha_{x} A_{1} e^{-\alpha_{x} x} \\
\pm k_{x} A_{2}\left[\begin{array}{c}
\sin \left(k_{x} x\right) \\
\cos \left(k_{x} x\right)
\end{array}\right] \\
\mp \alpha_{x} A_{1} e^{\alpha_{x} x}
\end{array}\right) e^{-i k_{z} z} \quad|x| \leq \frac{d}{2} \quad 2-31
$$

The boundary conditions require both tangential components of $\boldsymbol{E}$ and $\boldsymbol{H}$ to be continuous at the boundary $\boldsymbol{x}=\boldsymbol{d} / \mathbf{2}$ and $\boldsymbol{x}=-\boldsymbol{d} / \mathbf{2}$. Applying these conditions yields

$$
\begin{aligned}
& A_{1} e^{-\alpha_{x} d / 2}=A_{2} \cos \left(k_{x} d / 2\right) \\
& A_{1} e^{-\alpha_{x} d / 2}=\frac{k_{x}}{\alpha_{x}} A_{2} \sin \left(k_{x} d / 2\right)
\end{aligned}
$$

Combining Eq. 2-28 and Eq. 2-32, we have the even mode solution as:

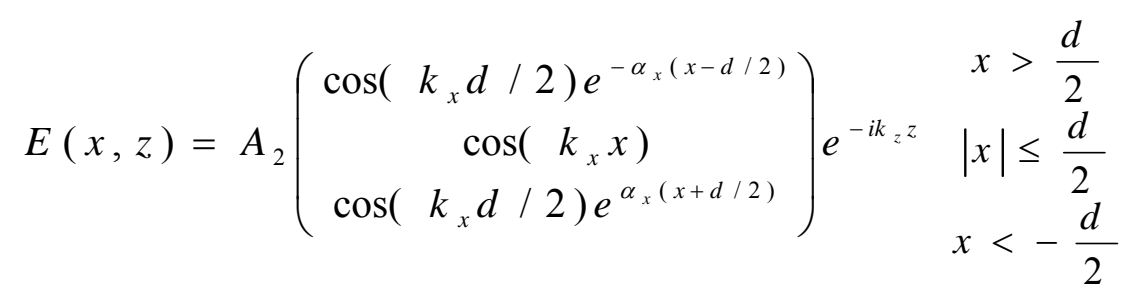

For odd modes, we have 


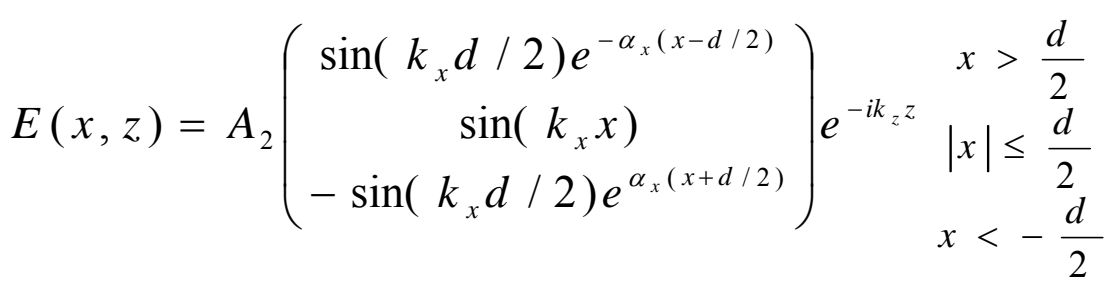

Also, for both Eq. 2-33 and Eq. 2-34 to be true, additional conditions arise.

$$
\begin{array}{ll}
\tan \left(k_{x} d / 2\right)=\alpha_{x} / k_{x} & \text { even modes } \\
\cot \left(k_{x} d / 2\right)=-\alpha_{x} / k_{x} & \text { odd modes }
\end{array}
$$

These two conditions are known as guidance conditions. Therefore, the modes are discrete. The number of modes in a particular waveguide depends on three refractive indices $\boldsymbol{n}_{\text {cladding }}, \boldsymbol{n}_{\text {waveguide, }}, \boldsymbol{n}_{\text {substrate }}$, the waveguide thickness $\boldsymbol{d}$, and the incident frequency $\omega$. When a similar analysis is performed to TM modes, we have guidance conditions as

$$
\begin{aligned}
& \tan \left(k_{x} d / 2\right)=\frac{n_{2}^{2}}{n_{1}^{2}} \alpha_{x} / k_{x} \text { even modes } \\
& \cot \left(k_{x} d / 2\right)=-\frac{n_{1}^{2}}{n_{2}^{2}} \alpha_{x} / k_{x} \text { odd modes }
\end{aligned}
$$

For the above even-mode equations, when $\left(k_{x}\right)_{m} d / 2 \rightarrow m \pi$, the attenuation factor $\alpha_{x}$ approaches 0 . This limit is well known as the cutoff limit (Lee, 1986; Snyder et al., 1988). Here, $\boldsymbol{m}$ means the $\boldsymbol{m}$ th mode.

\section{2-1-3-3 TE and TM modes in asymmetric dielectric waveguide}

Although the symmetric waveguide structure is particularly easy to analyze, the asymmetric waveguide is much more useful in practice. Asymmetric means the refractive indices of each layer are different $-\boldsymbol{n}_{\text {cladding }} \neq \boldsymbol{n}_{\text {substrate. }}$. This difference leads to a phase shift between the electric and magnetic fields in the waveguide. Based on the solutions obtained for symmetric waveguides, the TE modes (Lee, 1986) have electric fields described by: 


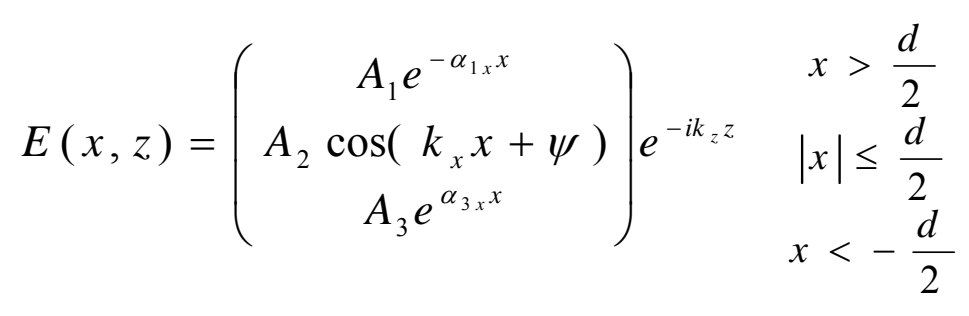

Here, these transverse parameters are given by a dispersion relation as

$$
\begin{aligned}
& k_{x}=\sqrt{\omega^{2} \mu_{2} \varepsilon_{2}-k_{z}^{2}} \\
& \alpha_{1 x}=\sqrt{k_{z}^{2}-\omega^{2} \mu_{1} \varepsilon_{1}} \\
& \alpha_{3 x}=\sqrt{k_{z}^{2}-\omega^{2} \mu_{3} \varepsilon_{3}}
\end{aligned}
$$

$\psi$ is a constant number which represents the phase shift. By taking the curl of Eq. 2-37, we get the magnetic field as:

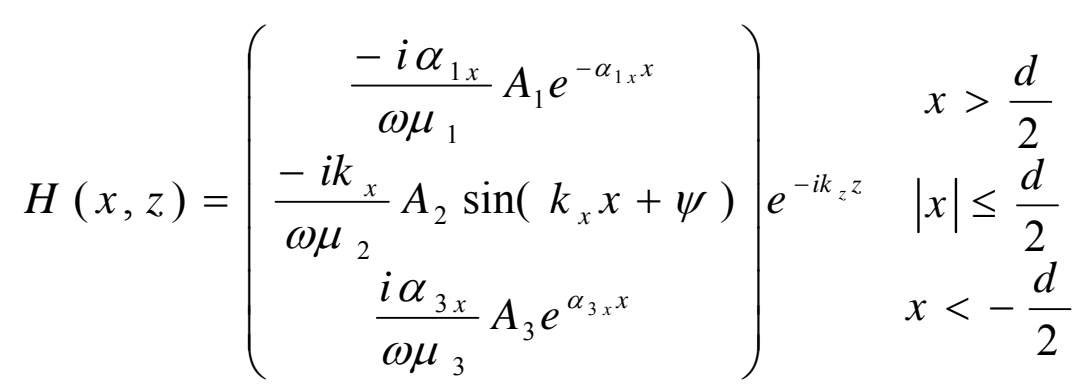

Following a similar approach as used for symmetric waveguides, the tangential components of $\boldsymbol{E}$ and $\boldsymbol{H}$ are continuous. Applying these boundary conditions at $\boldsymbol{x}=\boldsymbol{d} / \boldsymbol{2}$ yields:

$$
\begin{aligned}
& A_{1} e^{-\alpha_{1 x} d / 2}=A_{2} \cos \left(k_{x} d / 2+\psi\right) \\
& A_{1} e^{-\alpha_{1 x} d / 2}=\frac{\mu_{1} k_{x}}{\mu_{2} \alpha_{1 x}} A_{2} \sin \left(k_{x} d / 2+\psi\right)
\end{aligned}
$$

Taking the ratio of these two equations to eliminate $A_{1}$ and $A_{2}$ gives

$$
\tan \left(k_{x} d / 2+\psi\right)=\frac{\mu_{2} \alpha_{1 x}}{\mu_{1} k_{x}}
$$

Tangential components of $\boldsymbol{E}$ and $\boldsymbol{H}$ are also continuous at $\boldsymbol{x}=\boldsymbol{- d} / \mathbf{2}$, yields

$$
\tan \left(k_{x} d / 2-\psi\right)=\frac{\mu_{2} \alpha_{3 x}}{\mu_{3} k_{x}}
$$

By mathematical way to eliminate $\psi$, then we can get the guidance condition in an 
asymmetric waveguide:

$$
2 k_{x} d-\phi_{1}^{T E}-\phi_{3}^{T E}=2 m \pi \quad m=0,1,2 \ldots
$$

where

$$
\begin{aligned}
& \phi_{1}^{T E}=2 \tan ^{-1}\left(\mu_{2} \alpha_{1 x} / \mu_{1} k_{x}\right) \\
& \phi_{3}^{T E}=2 \tan ^{-1}\left(\mu_{2} \alpha_{3 x} / \mu_{3} k_{x}\right)
\end{aligned}
$$

The resulting electric fields are given by

$$
E(x, z)=A_{2}\left(\begin{array}{c}
\cos \left(k_{x} d / 2+\psi\right) e^{-\alpha_{1 x}(x-d / 2)} \\
\cos \left(k_{x} x+\psi\right) \\
\cos \left(k_{x} d / 2-\psi\right) e^{\alpha_{3 x}(x+d / 2)}
\end{array}\right) e^{-i k_{z} z} \quad \begin{array}{r}
|x| \leq \frac{d}{2} \\
x<-\frac{d}{2}
\end{array}
$$

where $\psi$ can be solved from Eq. 2-46 or Eq. 2-47.

For TM modes (Lee, 1986), the guidance condition can be obtained as

$$
2 k_{x} d-\phi_{1}^{T M}-\phi_{3}^{T M}=2 m \pi m=0,1,2 \ldots
$$

where

$$
\begin{aligned}
& \phi_{1}^{T M}=2 \tan ^{-1}\left(\varepsilon_{2} \alpha_{1 x} / \varepsilon_{1} k_{x}\right) \\
& \phi_{3}^{T M}=2 \tan ^{-1}\left(\varepsilon_{2} \alpha_{3 x} / \varepsilon_{3} k_{x}\right)
\end{aligned}
$$

and the magnetic field solution is

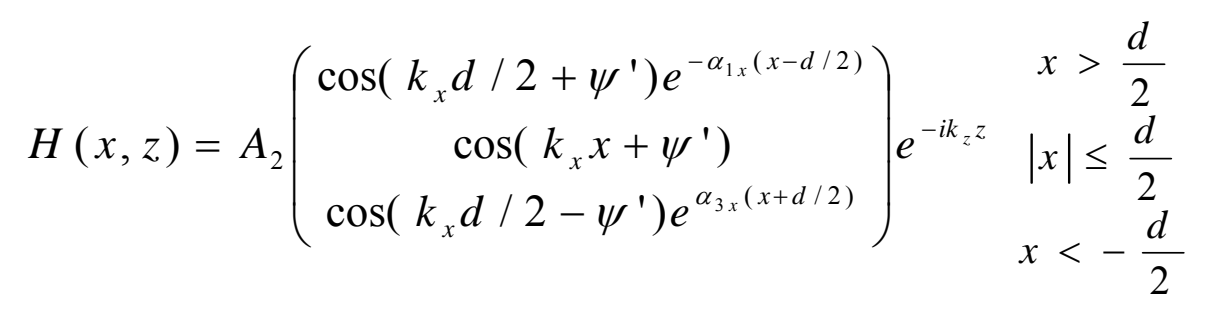

\section{2-1-3-4 Effective refractive index and dispersion relation in waveguide}

For light propagation in vacuum, the velocity and the wavevector $\boldsymbol{k}_{0}$ are $c=3.0 \times 10^{8} \mathrm{~m} / \mathrm{s}$ and $k_{0}=\omega \sqrt{\mu \varepsilon_{0}}=2 \pi / \lambda_{\text {vac }}$, respectively. Considering light propagation in dielectric materials, both velocity and wave vector change to $\boldsymbol{v}=\boldsymbol{c} / \boldsymbol{n}$ and $k=\omega \sqrt{\mu \varepsilon}=2 \pi n / \lambda_{\text {vac }}=n k_{0}$, where $\boldsymbol{n}$ is the refractive index of the dielectric material. Evanescent wave technique tells us that a small part of the light energy will 
enter into the substrate and cladding layers when light propagates in such a waveguide. Thus, when considering the velocity and wave vector in such a waveguide, we should use five parameters: refractive indices of substrate, film and cladding- $\boldsymbol{n}_{\text {substrate }}, \boldsymbol{n}_{\text {film }}, \boldsymbol{n}_{\text {cladding }}$, film thickness $d$ and incident wavelength or frequency. In this case, we define the propagation velocity as $\mathrm{v}=\boldsymbol{c} / \boldsymbol{n}_{\text {eff }}$ and propagation constant as $k_{z}=\omega \sqrt{\mu \varepsilon_{e f f}}=2 \pi n_{\text {eff }} / \lambda_{\text {vac }}=n_{\text {eff }} k_{0}$, where $\boldsymbol{n}_{\text {eff }}$ is known as the effective refractive index of a slab dielectric waveguide:

$$
n_{\text {eff }}=\sqrt{\varepsilon_{\text {eff }} / \varepsilon_{0}}
$$

The effective permittivity $\varepsilon_{\text {eff }}$ for each mode is given by the dispersion relation in the slab waveguide, as shown in Fig. 2.8 (Kogelnik et al., 1974). Here, we assume $\boldsymbol{n}_{\text {film }}>\boldsymbol{n}_{\text {substrate }}>\boldsymbol{n}_{\text {cladding. }}$

In the dispersion relation graphs shown in Fig 2.8, a frequency parameter $v$ is normalized to film thickness $\boldsymbol{d}$ and is defined as

$$
v=k_{0} d \sqrt{\left(\varepsilon_{2}-\varepsilon_{3}\right) / \varepsilon_{0}}
$$

Next, a parameter $b$ is defined by

$$
b=\left(\varepsilon_{\text {eff }}-\varepsilon_{\text {substrate }}\right) /\left(\varepsilon_{\text {film }}-\varepsilon_{\text {substrate }}\right)
$$

From the above equation, if $\mathrm{b}$ and the refractive indices of substrate and film are known, we will get an effective permittivity and effective refractive index by

$$
\begin{aligned}
& \varepsilon_{\text {eff }}=b\left(\varepsilon_{\text {film }}-\varepsilon_{\text {substrate }}\right)+\varepsilon_{\text {substrate }} \\
& n_{\text {eff }}=\sqrt{n_{\text {substrate }}{ }^{2}+b\left(n_{\text {film }}{ }^{2}-n_{\text {substrate }}{ }^{2}\right)}
\end{aligned}
$$

The other parameter $\boldsymbol{a}$ is the asymmetric measure. The value of this parameter is slightly different between TE modes and TM modes.

$$
\begin{aligned}
& a^{T E}=\left(\varepsilon_{\text {substrate }}-\varepsilon_{\text {cladding }}\right) /\left(\varepsilon_{\text {film }}-\varepsilon_{\text {substrate }}\right) \\
& a^{T M}=\left(\frac{\varepsilon_{\text {film }}}{\varepsilon_{\text {cladding }}}\right)^{2}\left(\varepsilon_{\text {substrate }}-\varepsilon_{\text {cladding }}\right) /\left(\varepsilon_{\text {film }}-\varepsilon_{\text {substrate }}\right)
\end{aligned}
$$

For the symmetric waveguide, refractive indices of substrate and cladding are equal, which yields $\boldsymbol{a}=\boldsymbol{0}$. 


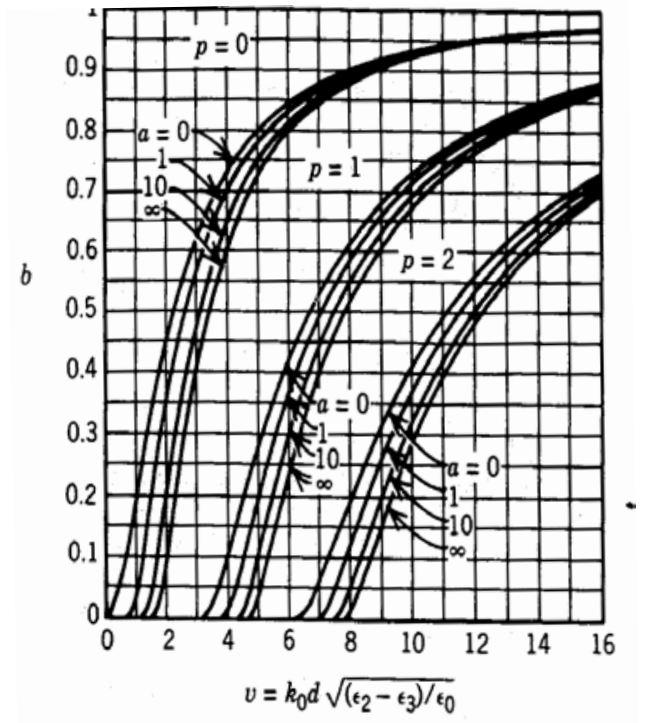

(a)

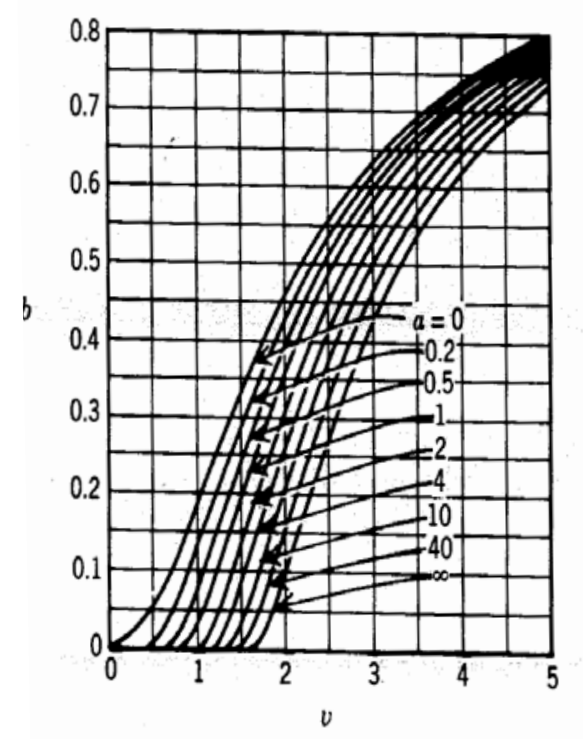

(b)

Fig. 2.8. Normalized $b$-v graph. (a) First three modes for several values of $a$

(b) Expanded plot for the fundamental mode (Kogelnik et al., 1974).

\section{2-2 Mode coupling in a directional coupler}

When two waveguides are brought into close proximity, the power carried by these waveguides may periodically exchange between them with distance. If the modes in these two waveguides are always traveling in a forward direction, then we call this forward coupling. A forward coupling device is also called a directional coupler. Directional couplers are used in a wide variety of applications, not only for SPARROW biosensor transducer but also for optical fiber switching network, line monitoring, power measurements, load source isolators, etc. Mode coupling in a directional coupler is the key to understanding how the transducer transmits a biosignal. This will be explained next.

\section{2-2-1 Coupling coefficient}

Assuming a waveguide is homogenous in the $\boldsymbol{y}$ direction, the general wave equation is 


$$
\nabla^{2} E+\nabla\left[\frac{E \cdot \nabla n^{2}}{n}\right]=\frac{1}{C^{2}} \frac{\partial^{2} n^{2} \cdot E}{\partial t^{2}}+\mu \frac{\partial J}{\partial t}
$$

where $\boldsymbol{n}=\boldsymbol{n}_{0}(\boldsymbol{x})$ is the waveguide refractive index and $\boldsymbol{C}$ is the light velocity in vacuum. In source-free condition, $\boldsymbol{J}=\mathbf{0}$, we can get the steady-state wave equation

$$
\frac{\partial^{2} E}{\partial z^{2}}+H_{t} E=-k_{0} n^{2} E
$$

where

$$
H_{t} E=\nabla_{t}^{2} E+\nabla\left[\frac{E \cdot \nabla n^{2}}{n^{2}}\right]
$$

Here, the subscript $\boldsymbol{t}$ means in the traverse or in $\boldsymbol{x}$ direction. The general solution for Eq. $2-61$ is

$$
E(x, z)=\sum_{m} a_{m} E(x) e^{-i k_{z m} z}
$$

The parameter $\boldsymbol{m}$ gives how many waveguides that are present. $\boldsymbol{a}_{\boldsymbol{m}}$ is the $\boldsymbol{m}$ th amplitude and $\boldsymbol{k}_{z \boldsymbol{m}}$ is the $\boldsymbol{m}$ th propagation constant.

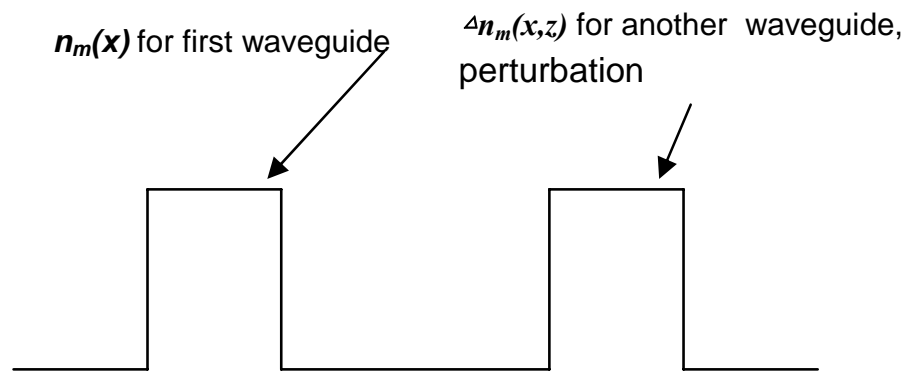

Fig. 2.9. Coupled waveguide indices.

Consider that one waveguide index is a perturbation of another waveguide index, as shown in Fig. 2.9. The perturbated refractive index can be rewritten as

$$
n(x, z)=n_{m}(x)+\Delta n_{m}(x, z)
$$

where $\boldsymbol{n}_{\boldsymbol{m}}(\boldsymbol{x})$ is the first waveguide effective index before perturbation, and $\boldsymbol{\Delta n}_{\boldsymbol{m}}(\boldsymbol{x}, \boldsymbol{z})$ is the second waveguide effective index (Perturbation term). Substitute this $\boldsymbol{n}(\boldsymbol{x}, \boldsymbol{z})$ into the wave equation and assuming $\boldsymbol{a}_{\boldsymbol{m}}$ varies very slowly with distance, the wave equation becomes 


$$
\sum_{m}\left(-2 i k_{z m} \frac{\partial a_{m}}{\partial z}+\left(k_{0}^{2} n^{2}-k_{z m}^{2}\right) a_{m}+\hat{H}_{t} a_{m}\right) E_{m}(x) e^{-i k_{z m} z}=0
$$

Mathematically, we can overlap with $\left\langle E_{n}(x, y) e^{-i k_{n n}{ }^{2}}\right|$ and expand this equation to give

$$
\sum_{n}\left[\begin{array}{l}
\left\langle E_{n}(x) e^{-i k_{z n} z}\left|-2 i k_{z m} \frac{\partial a_{m}}{\partial z}\right| E_{m}(x) e^{-i k_{z m} z}\right\rangle \\
+\left\langle E_{n}(x) e^{-i k_{z n} z}\left|\left(H_{t}+k_{0}{ }^{2} n^{2}-k_{z m}{ }^{2}\right) a_{m}\right| E_{m}(x) e^{-i k_{z m} z}\right\rangle
\end{array}\right]=0
$$

If two waveguides are orthogonal $-\boldsymbol{m} \neq \boldsymbol{n}$, physically, this means that the electric fields of the two waveguides do not affect each other.

$$
\left\langle E_{m} \mid E_{n}\right\rangle=0
$$

Then, we can get the coupled wave equation as

$$
\frac{\partial a_{m}}{\partial z}+i \sum_{n} e^{i\left(k_{z m}-k_{z n}\right) z} C_{m n} a_{n}=0
$$

where

$$
C_{m n}=\left\langle E_{m}(x)\left|\frac{k_{0}{ }^{2} n_{n} \Delta n_{n}}{k_{z m}}\right| E_{n}(x)\right\rangle
$$

$\boldsymbol{C}_{\boldsymbol{m} \boldsymbol{n}}$ here is known as the coupling coefficient.

\section{2-2-2 Synchronous directional coupler}

If the two waveguides are identical, or synchronous, the propagation constants of the two waveguides are equal $\boldsymbol{k}_{z 1}=\boldsymbol{k}_{z 2}=\boldsymbol{k}_{z}$ and the coupling coefficients can be written as

$$
\begin{aligned}
& C_{11}=\left\langle E_{1}(x)\left|\frac{k_{0}{ }^{2} n_{1} \Delta n_{1}}{k_{z 1}}\right| E_{1}(x)\right\rangle \\
& C_{22}=\left\langle E_{2}(x)\left|\frac{k_{0}{ }^{2} n_{2} \Delta n_{2}}{k_{z 2}}\right| E_{2}(x)\right\rangle \\
& C_{12}=\left\langle E_{1}(x)\left|\frac{k_{0}{ }^{2} n_{2} \Delta n_{2}}{k_{z 1}}\right| E_{2}(x)\right\rangle \\
& C_{21}=\left\langle E_{2}(x)\left|\frac{k_{0}{ }^{2} n_{1} \Delta n_{1}}{k_{z 2}}\right| E_{1}(x)\right\rangle
\end{aligned}
$$

where $\boldsymbol{C}_{12}=\boldsymbol{C}_{21}=\boldsymbol{C}$ because $n_{1}=n_{2}$ and $\Delta n_{1}=\Delta n_{2}$. Therefore, the coupled mode 
equations become

$$
\begin{aligned}
& \frac{\partial a_{1}}{\partial z}=-i C_{11} a_{1}-i C a_{2} \\
& \frac{\partial a_{2}}{\partial z}=-i C_{22} a_{2}-i C a_{1}
\end{aligned}
$$

$\boldsymbol{C}_{11}$ and $\boldsymbol{C}_{12}$ are self coupling terms. Since they are much smaller than C, they can be ignored

$$
\begin{aligned}
& \frac{\partial a_{1}}{\partial z}=-i C a_{2} \\
& \frac{\partial a_{2}}{\partial z}=-i C a_{1}
\end{aligned}
$$

The solution of this equation is

$$
\begin{aligned}
& a_{1}=A_{11} e^{i C z}+A_{12} e^{-i C z} \\
& a_{2}=A_{21} e^{i C z}+A_{22} e^{-i C z}
\end{aligned}
$$

If we assume that power is injected into one waveguide at the input end (See Fig. 2.10), the initial conditions will be

$$
\begin{aligned}
& a_{1}(0)=1 \\
& a_{2}(0)=0
\end{aligned}
$$

The solution can be written as

$$
\begin{aligned}
& a_{1}=\operatorname{Cos}(\mathrm{Cz}) e^{-i k_{z} z} \\
& a_{2}=-i \operatorname{Sin}(\mathrm{Cz}) e^{-i k_{2} z}
\end{aligned}
$$
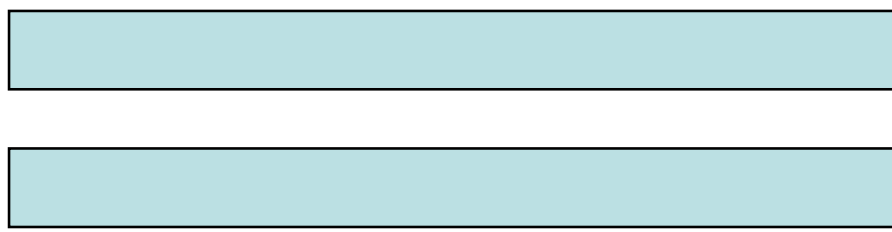

$Z=0$

Fig. 2.10. Synchronous directional coupler.

Correspondingly, the power in both waveguides will be given by 


$$
\begin{aligned}
& P_{1}=P_{0} \cos ^{2}(C z) \\
& P_{2}=P_{0} \sin ^{2}(C z)
\end{aligned}
$$

Figure 2.11 shows the power exchange in a synchronous directional coupler assuming a total power normalized to 1 .

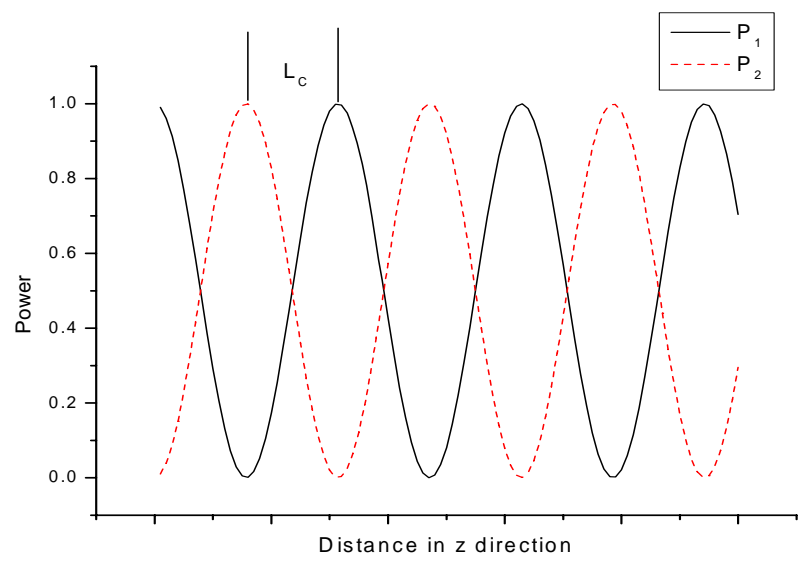

Fig. 2.11. Power in synchronous directional coupler.

Here, the coupling length $\boldsymbol{L}_{\boldsymbol{c}}$ is defined as half the oscillation period of the power

$$
L_{c}=\pi / 2 C
$$

At this distance, the power from one waveguide is completely transferred into the second waveguide. When $\boldsymbol{z}=\boldsymbol{L}_{\boldsymbol{c}}$, there is $100 \%$ power transfer and the power transfer efficiency is $100 \%$. This is a consequence of the directional coupler being synchronous. It has been described by many authors (Huang et al., 1994; Streifer, 1987, 1988; Vassello, 1986, 1988; Miller, 1954; Yariv, 1973; Tseng, 1999) and applied in numerous devices (Luff et al., 1996, 1998; Ctyroky et al., 1999; Sangeeta, 1995).

\section{2-2-3 Asynchronous directional coupler}

In the previous section, a synchronous directional coupler was discussed. However, if the two waveguides are not perfectly symmetric or are asynchronous (Lee, 1986; Yong et al., 2001; Huang, 1994), then we have $\boldsymbol{k}_{z 1} \neq \boldsymbol{k}_{z 2}$ and $\boldsymbol{C}_{\boldsymbol{1 2}} \neq \boldsymbol{C}_{\mathbf{2 1}}$. The wave equations then become 


$$
\begin{aligned}
& \frac{\partial a_{1}}{\partial z}=-i C_{12} a_{2} e^{i\left(k_{z 1}-k_{z 2}\right) z} \\
& \frac{\partial a_{2}}{\partial z}=-i C_{21} a_{1} e^{i\left(k_{z 2}-k_{z 1}\right) z}
\end{aligned}
$$

After mathematical manipulation, the solution for the above equation is

$$
\begin{aligned}
& a_{1}=e^{i \Delta z}\left[\cos (\gamma z)-i \frac{\Delta}{\gamma} \sin (\gamma z)\right] \\
& a_{2}=e^{-i \Delta z}\left[\frac{C}{\gamma} \sin (\gamma z)\right]
\end{aligned}
$$

where

$$
\begin{aligned}
& \Delta=\frac{k_{z 1}-k_{z 2}}{2} \\
& \gamma=\sqrt{\Delta^{2}+C_{12} C_{21}}=\sqrt{\Delta^{2}+C^{2}} \\
& C=\sqrt{C_{12} C_{21}}
\end{aligned}
$$

Using the initial conditions of Eq. 2-78, the power in each waveguide can be described as

$$
\begin{aligned}
& P_{1}=\left|a_{1}(z)\right|^{2}=1-F \sin ^{2}(\gamma z) \\
& P_{2}=\left|a_{2}(z)\right|^{2} \frac{k_{z 2}}{k_{z 1}}=F \sin ^{2}(\gamma z)
\end{aligned}
$$

where $\boldsymbol{F}$ is the power coupling efficiency defined as

$$
F=\frac{1}{1+(\Delta / C)^{2}}
$$

When two waveguides are synchronous, $\Delta=0$ and $F=1$, and the above expressions reduce to the synchronous case. Figure 2.12 shows the power in an asynchronous directional coupler.

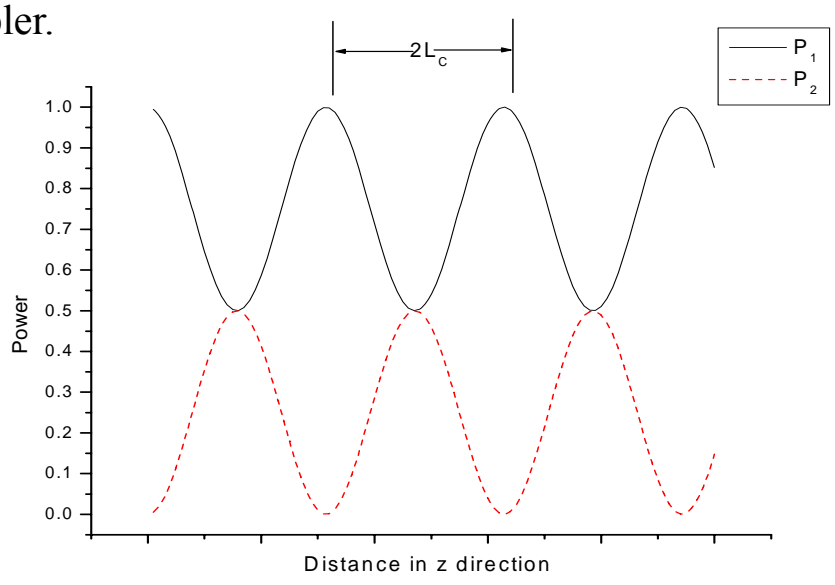

Fig.2.12. Power in asynchronous directional coupler. 


\section{2-2-4 Non-orthogonal coupled mode theory}

In the previous sections, the coupling length, coupling coefficient and coupling efficiencies were derived. Generally, this method is referred to as conventional coupledmode theory. This approach is expected to be valid under the weak-coupling limit (Hardy et al., 1988; Snyder et al., 1988). The electric fields in the two waveguide layers do not affect each other. However, for a more rigorous approach, interference between the two waveguides has to be considered. This method is called the non-orthogonal coupled mode theory (Huang, 1994; Huas et al., 1987, 1989, 1991). In this case, the electric fields in the two waveguides will affect each other due to interference. Mathematically, this takes the form

$$
\left\langle E_{m} \mid E_{n}\right\rangle=x \neq 0
$$

so the wave equation is changed to

$$
\sum_{n}\left[\begin{array}{l}
-2 i e^{-i k_{2 n} z} X_{m n} \frac{\partial \beta_{n} a_{n}}{\partial z} \\
+e^{-i k_{z n} z}\left\langle E_{m}(x)\left|k_{0}^{2}\left(n^{2}-n_{n}^{2}\right)\right| E_{n}(x)\right\rangle a_{n}
\end{array}\right]=0
$$

where

$$
X_{m n}=\left(\begin{array}{ll}
1 & x \\
x & 1
\end{array}\right)
$$

Using the matrix representation, we get

$$
X \frac{\partial A}{\partial z}=-i H A
$$

where

$$
\begin{aligned}
& H_{m n}=X_{m n} k_{z n}+C_{m n} \\
& A_{m n}=k_{z n} a_{n} e^{-i k_{z n} z}
\end{aligned}
$$

To solve this matrix equation, we introduce a transformation matrix $\boldsymbol{O}$. Let

$$
\begin{aligned}
& O^{+} X O=I \\
& O^{+} H O=\left(\begin{array}{cc}
\beta_{s} & 0 \\
0 & \beta_{a}
\end{array}\right)
\end{aligned}
$$


Combine the above equations and the transformation matrix $\boldsymbol{O}$ can be written as

$$
O=\frac{1}{\cos \alpha}\left(\begin{array}{cc}
\cos \left(\frac{\phi+\alpha}{2}\right) & -\sin \left(\frac{\phi+\alpha}{2}\right) \\
\sin \left(\frac{\phi-\alpha}{2}\right) & \cos \left(\frac{\phi-\alpha}{2}\right)
\end{array}\right)
$$

where

$$
\sin \alpha=x
$$

and

$$
\tan \phi=\frac{C_{12}+C_{21}-x\left(C_{11}+C_{22}\right)}{k_{z 1}-k_{z 2}+C_{11}-C_{22}}
$$

Therefore, propagation constants $\boldsymbol{\beta}_{\boldsymbol{s}}$ and $\boldsymbol{\beta}_{\boldsymbol{a}}$ in coupled waveguides can be derived as

$$
\beta_{s, a}=\frac{\frac{H_{11}+H_{22}}{2}-x H_{12}}{1-x^{2}} \pm \sqrt{\frac{1}{1-x^{2}}\left(x \frac{H_{11}+H_{22}}{2}-H_{12}\right)^{2}+\frac{\left(\frac{H_{11}-H_{22}}{2}\right)^{2}}{1-x^{2}}}
$$

The coupling length is now

$$
L_{c}=\frac{\pi}{\beta_{s}-\beta_{a}}
$$

and the power in each waveguide is

$$
\begin{aligned}
& P_{1}=P_{0}\left|A_{1}\right|^{2} / k_{z 1} \\
& P_{2}=P_{0}\left|A_{2}\right|^{2} / k_{z 2}
\end{aligned}
$$

where

$$
A_{n}(x)=O\left(\begin{array}{cc}
e^{-i \beta_{s} z} & 0 \\
0 & e^{-i \beta_{a} z}
\end{array}\right) O^{-1} A_{n}(0)
$$

If the waveguides are synchronous, these equations reduce to a maximum value of $\left|A_{2}\right|^{2} / k_{z 2}=1$. Thus, the power transfer efficiency is $100 \%$. If the waveguides are asynchronous, the maximum value of $\left|A_{2}\right|^{2} / k_{z 2}<1$, and only part of the power can be transferred to another waveguide. 


\section{Chapter 3}

\section{Modeling of ideal SPARROW biosensor structure}

In reality, a biosensor device can suffer from serious problems like material inhomogenuity and surface roughness that can cause lot of difficulties. To approach real device modeling, it is necessary to build an ideal structure model first. An ideal SPARROW biosensor structure assumes that there is no power loss during light propagation, layers are homogenous, and surface roughness is zero. Such an ideal model can help serve as the basis for understanding light propagation in real devices. Also, it can be used to help optimize device performance. In this work, two methods were used to build the SPARROW biosensor ideal model. One is a computer simulation which is based on Finite Difference Beam Propagation Method (FD-BPM). The other is an analytical expression based on coupled mode theory described in section 2-2.

\section{3-1 Beam Propagation Method}

The Beam Propagation Method (BPM), as the kernel of computer simulation programs, is one of the most powerful techniques to investigate the light wave propagation in different shapes of waveguides and optical fibers (OptiBPM manual). The key to simulation is decomposing the spatial mode into the superposition of plane waves, each traveling in slightly different directions. After traveling through the whole dielectric medium, these plane waves will be added back together. Each optical plane wave will be subjected to diffraction and will have a slight phase shift when propagating from one position to another adjacent position. In addition, the refractive index of short propagation must be considered. To analyze how light propagates between these positions, the medium was treated as a sequence of lenses by short sections of homogenous space (Marcuse, D., 1973). Figure 3.1 shows the optical path broken into such a sequence of lenses. 


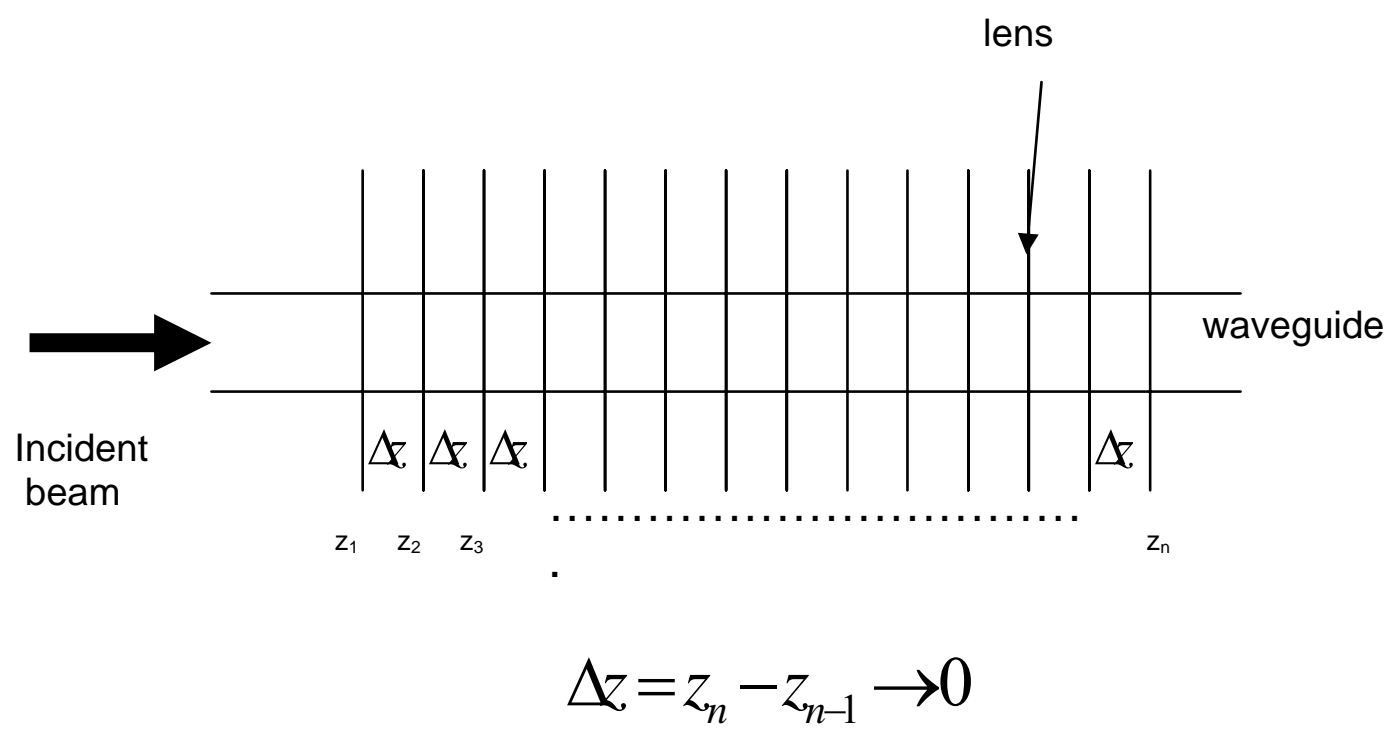

Fig. 3.1. Optical path broken into sequence of lenses (Marcuse, D., 1973).

So far, several kinds of beam propagation methods which are based on different mathematic approaches are developed. In our simulation software-OptiBPM, finite difference BPM (FD-BPM) is used. It solves Maxwell's equations by using finite differences instead of partial derivatives (OptiBPM manual; Optiwave manual). In this sense, FD-BPM can be accurate in a wide range of devices. However, there are two assumptions which limit the application range of this method. First, this method assumes that the device has an optical axis so that most of the light travels in this direction or approximately in this direction. Second, a slow-varying envelope approximation is used (also used in coupled mode theory). In Ch. 2, Eq. 2.69 gives $E(x, z)=\sum_{m} a_{m} E(x) e^{-i k_{z m} z}$,

where $\boldsymbol{a}_{\mathrm{m}}$ is the $\boldsymbol{m}$ th amplitude. If one assumes $\boldsymbol{a}_{\mathrm{m}}$ varies slowly with distance, then the second order differential, $\frac{\partial^{2} a_{m}}{\partial z^{2}}$, can be ignored when solving wave equations. For many practical waveguides, including the SPARROW biosensor, the refractive contrast in the transverse direction is small. Thus, $\boldsymbol{a}_{\mathrm{m}}$ varies slowly in the transverse direction and approximation should works well. 


\section{3-2 Modes in single planar waveguide}

Based on FD-BPM, a computer simulation offers a quick way to simulate the light propagation in waveguides. Especially when using the mode solver in this program, it gives the propagation constants, effective index, and modal fields of waveguides. These parameters will help us optimize the waveguides such as determining waveguide thickness, separation distance between waveguides, mode type of incident beam, etc. To apply the computer simulation on a SPARROW biosensor, the simulation is first applied to each individual waveguide.

Top waveguide

Cladding

$n=1.3318$ bottom waveguide

Cladding

$n=1.457$

\begin{tabular}{cc}
\hline Waveguide $\boldsymbol{n}=1.659$ & Waveguide $\boldsymbol{n}=1.659$ \\
\hline $\begin{array}{l}\text { Substrate } \\
\boldsymbol{n}=1.457\end{array}$ & $\begin{array}{l}\text { Substrate } \\
\boldsymbol{n}=1.4701\end{array}$
\end{tabular}

Fig. 3.2. Top and bottom waveguides in a SPARROW biosensor.

Figure 3-2 shows the top and bottom waveguides in this device separately. Since this device will be operated in a water environment, we assume the refractive index of the cladding is 1.3318 , i.e., the refractive index of water at $633 \mathrm{~nm}$. Changing the waveguide thickness will change the effective refractive index, modal shape, and mode number. In addition, the TE and TM modes will exhibit different behaviors. Figure 3.3 shows a TE mode and a TM mode in a single waveguide layer.

Generally, we can use either a TE or TM mode in our device. Though their behaviors are not the same, there is not much difference when doing simulations and mathematic calculations. To simplify the approach, only TE modes are considered. Also, we will use TE modes in the actual device operation. As stated previously, the number of TE modes in single waveguide and the mode shape depend on the waveguide thickness when incident wavelength and indices of waveguide are fixed. 
Optical Field

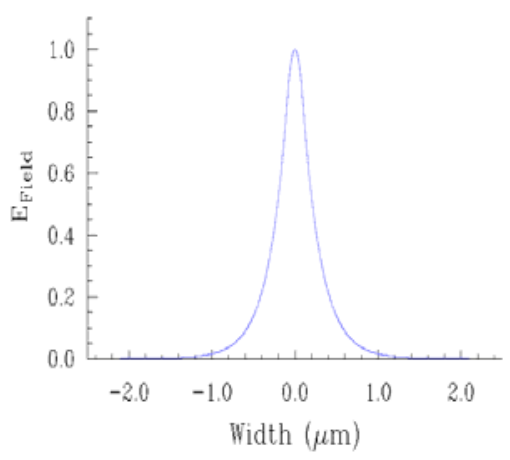

Single TE mode
Optical Field

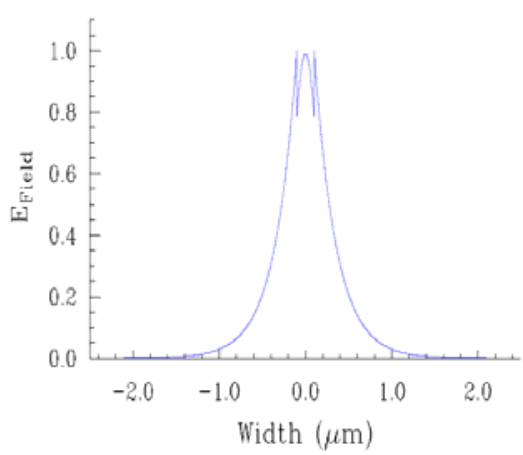

Single TM mode

Fig. 3.3. Single TE and TM mode in a single waveguide layer.

From the computer simulation, if the thickness of the bottom waveguide is less than 38 $\mathrm{nm}$, there will be no propagating mode. If the waveguide thickness is greater than 447 $\mathrm{nm}$, then there will be two modes supported by the waveguide. Multiple modes will increase the complexity for both the simulation and the real device. To ensure a single mode in the bottom waveguide, the thickness must then be limited to between $38 \mathrm{~nm}$ and $447 \mathrm{~nm}$. For a single propagating mode in the top waveguide, the range of thickness is from $94 \mathrm{~nm}$ to $484 \mathrm{~nm}$. Figures 3.4 and 3.5 show how the mode shape changes with variations in thickness of the waveguide layers.

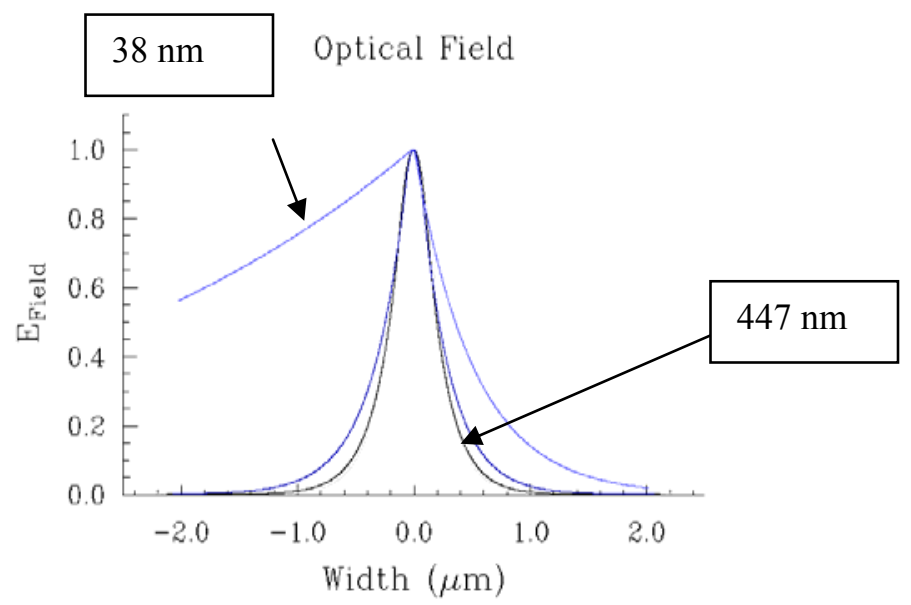

Fig. 3.4. Effect of thickness variation on modes in bottom waveguide. 


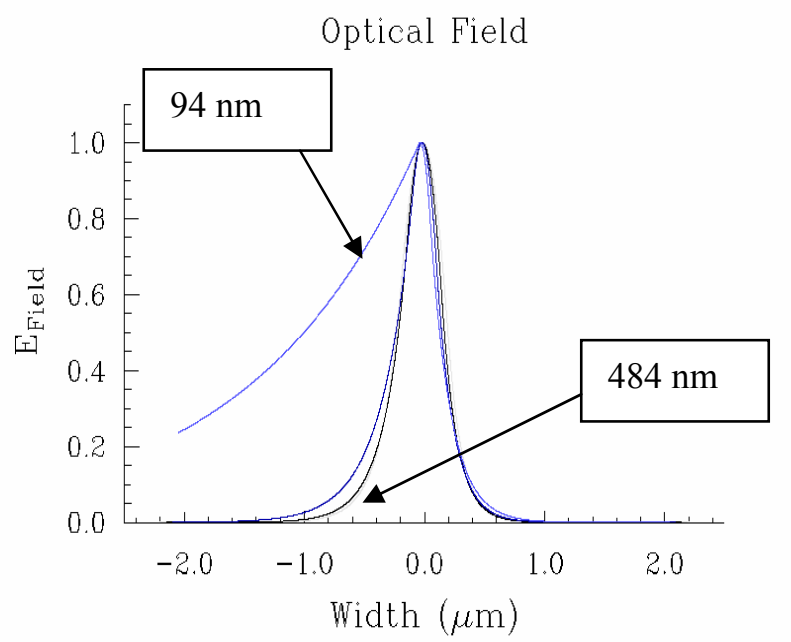

Fig. 3.5. Effect of thickness variation on modes in top waveguide.

In the SPARROW biosensor (Fig. 1.3), the top waveguide is 194-nm thick and the bottom waveguide is 144-nm thick. Due to fabrication difficulties, it may be hard to achieve these exact thicknesses, but a waveguide that supports only a single mode should be acceptable. In addition, to meet with the phase-matching requirements, both waveguides thicknesses depend on each other. This will be discussed in section 3-3.

In a real device, not only the thickness but also the refractive index of each layer could vary. Thus, it is necessary to consider the effect of refractive index variation. Compared to the index of $\mathrm{Al}_{2} \mathrm{O}_{3}$, the indices of $\mathrm{SiO}_{2}$ and Borofloat glass will vary only slightly, which we can ignore. However, ellipsometry measurements at $633 \mathrm{~nm}$ have shown that the refractive index of the alumina layer could vary from 1.635 to 1.660 . Results of the computer simulation are shown in Fig. 3.6 and Table 3.1 for changes in mode and the effective index values for this range in $\mathrm{Al}_{2} \mathrm{O}_{3}$ index values. For this simulation, $144 \mathrm{~nm}$ was used for the bottom waveguide thickness and $194 \mathrm{~nm}$ for the top waveguide thickness. 

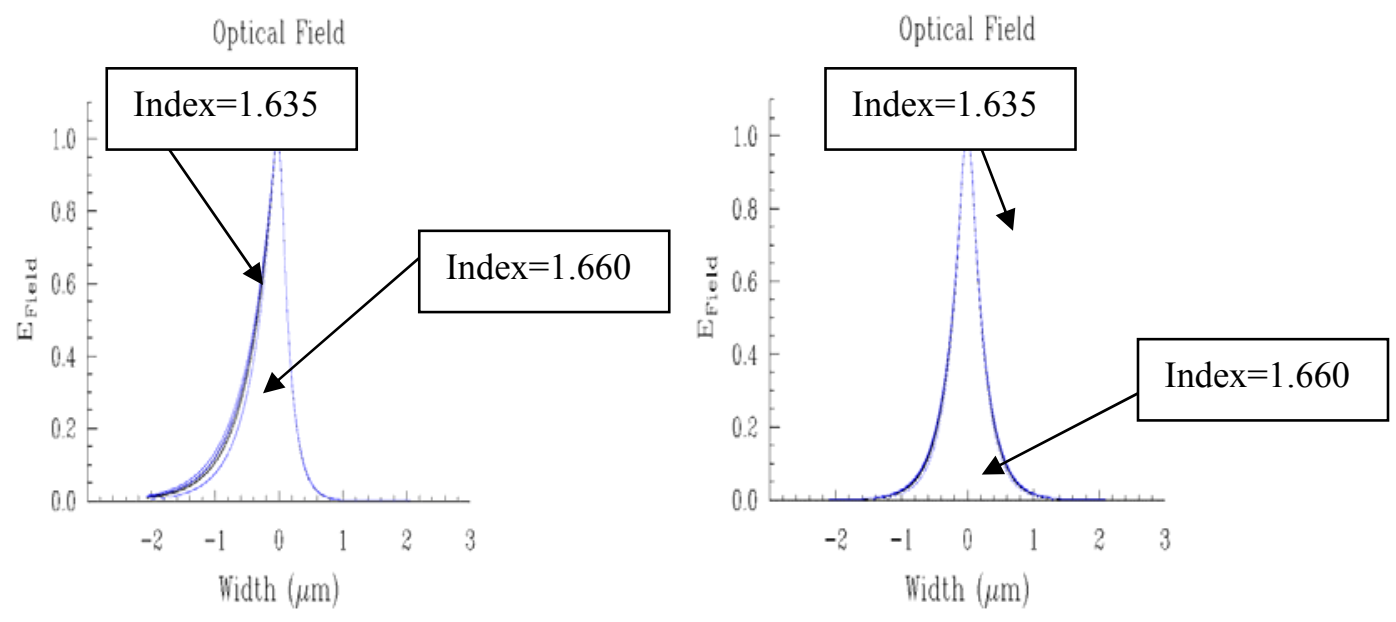

Fig. 3.6 Modes in waveguide with refractive index variation.

Table 3.1. Effective refractive index variations for TE mode.

\begin{tabular}{ccc}
\hline Waveguide index & $\begin{array}{c}\text { Top waveguide } \\
\text { effective index }\end{array}$ & $\begin{array}{c}\text { Bottom waveguide } \\
\text { effective index }\end{array}$ \\
\hline 1.635 & 1.4956 & 1.4999 \\
\hline 1.640 & 1.4984 & 1.5019 \\
\hline 1.645 & 1.5013 & 1.5038 \\
\hline 1.650 & 1.5043 & 1.5059 \\
\hline 1.655 & 1.5073 & 1.5079 \\
\hline 1.660 & 1.5104 & 1.5100 \\
\hline
\end{tabular}

\section{3-3 Modes and light propagation in coupled waveguide}

When two waveguides are placed together, the optical power will exchange periodically between the two layers. This behavior is called light coupling in waveguides. A computer simulation can be used to determine the modes in these two waveguides, the coupling length, the maximum power transfer, etc. Figure 3.7 shows the computer simulation for two identical waveguides. 
$\mathrm{n}=1$

\begin{tabular}{ll}
\hline Waveguide $1 \mathrm{n}=1.5 \mathrm{~d}=200 \mathrm{~nm}$ \\
\hline $\mathrm{n}=1 \mathrm{~d}=600 \mathrm{~nm}$ \\
\hline Waveguide $2 \mathrm{n}=1.5 \mathrm{~d}=200 \mathrm{~nm}$ \\
\hline $\mathrm{n}=1$ \\
Coupled Waveguides
\end{tabular}

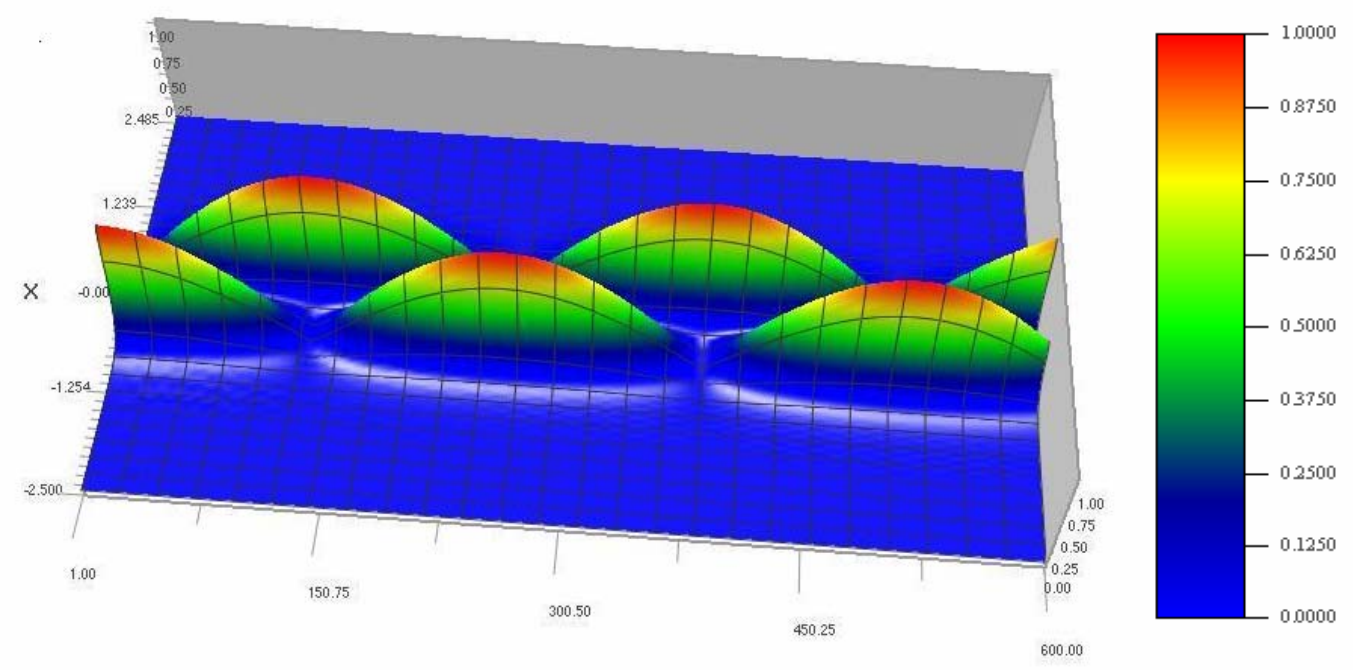

Fig. 3.7. Light intensity in coupled waveguides (wavelength $=633 \mathrm{~nm}$ ).

In Fig. 3.7, the power enters in the bottom waveguide and after some distance, all power transfers to the top waveguide. This software package can not determine the exact coupling length, but from the graph, a rough estimate of $127 \mu \mathrm{m}$ is obtained. The power transfer efficiency between these two waveguides is $100 \%$.

Next, a computer simulation for an ideal SPARROW biosensor transducer is performed. Although the thicknesses of these two waveguides are not the same, their effective refractive indices are equal, which means the propagation constants are the same. So, the power transfer efficiency is still $100 \%$ between these two waveguides. From Fig. 3.8, the coupling length is $186 \mu \mathrm{m}$. Also, we can get the supermodes in these two waveguides as shown in Fig. 3.9. There are two modes that exist in these coupled 
waveguides, one is

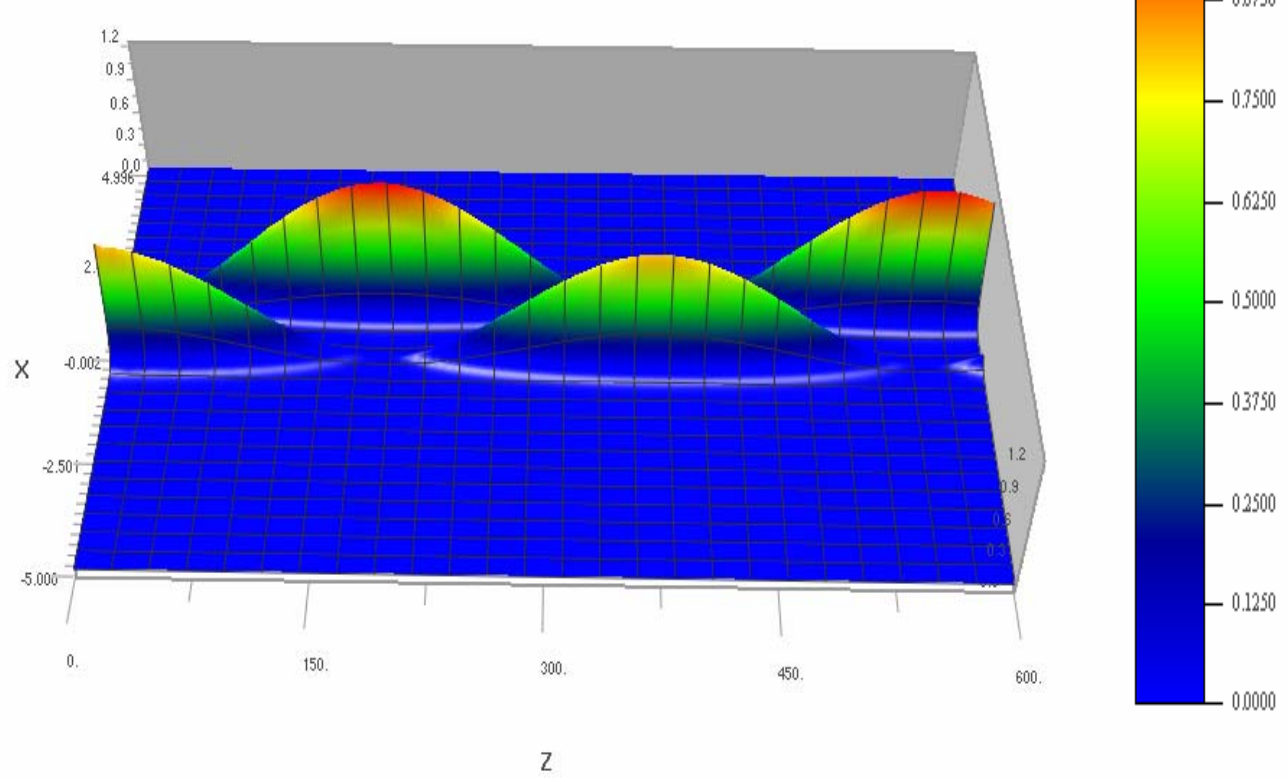

Fig 3.8. Light intensity in ideal SPARROW biosensor waveguide.

Optical Field

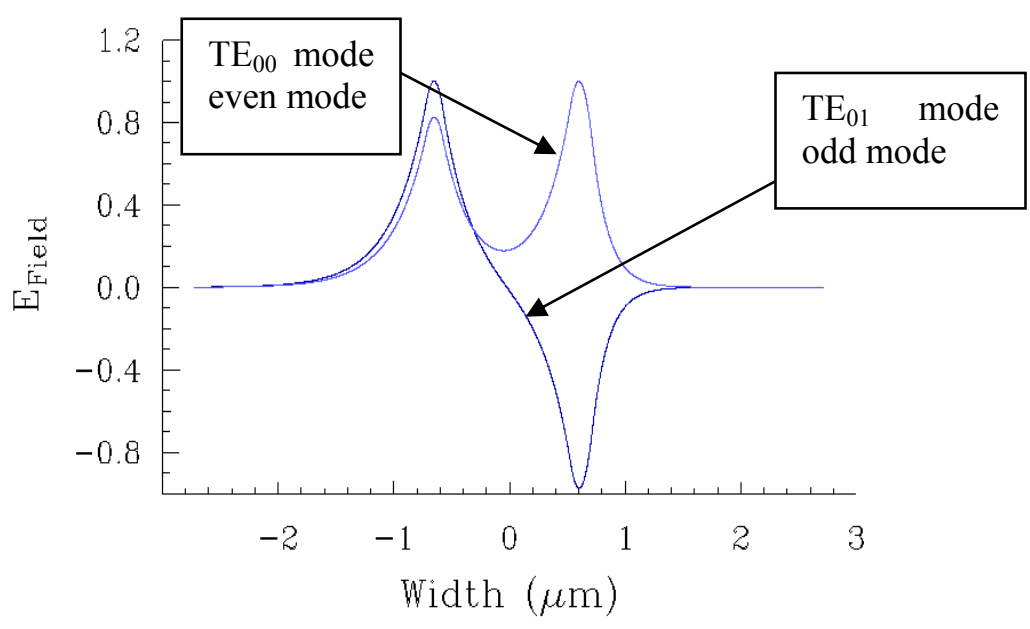

Fig. 3.9. Supermodes in coupled waveguide. 
a $\mathrm{TE}_{00}$ mode (even mode). The other mode is $\mathrm{TE}_{01}$ (odd mode). The modal index values are 1.5105 and 1.5088 . If the propagation constants of these two waveguides are different, the coupling length and power transfer efficiency will change. Figure 3.10 shows this change with mismatched propagation constants which may occur in real devices.

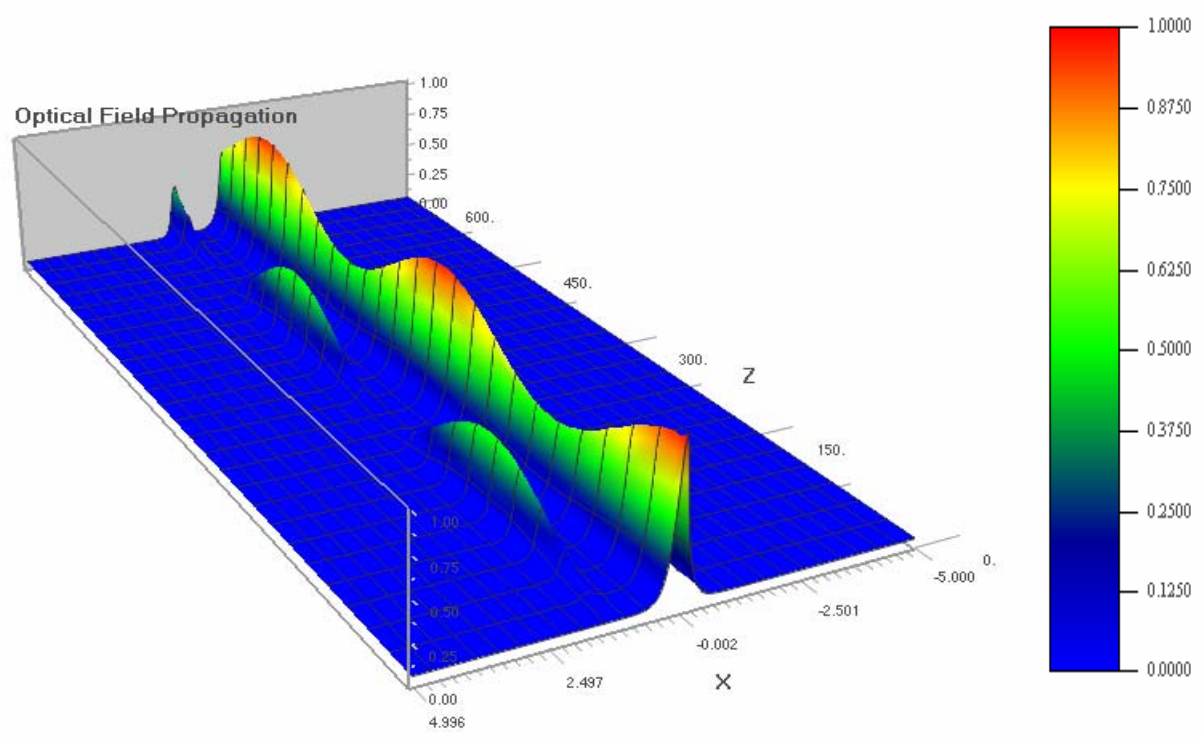

Fig 3.10. Light intensity in coupled waveguides.

From Fig. 3.10, only partial power would transfer to the second waveguide. The coupling length is also reduced. Compared with the ideal structure, we only change top waveguides thickness from $194 \mathrm{~nm}$ to $190 \mathrm{~nm}$. So phase matching is important in our device design. Generally, before depositing top waveguide layer, we should check the thicknesses and refractive indices of other three layers (Borofloat, bottom waveguide, silicon oxide) to get the actual value. Then, by mathematical calculation or computer simulation, we can get the best thickness value for the top waveguide. This can help direct the deposition of the top layer.

Using a mode solver, we can plot a graph to see how thickness and refractive index variations affect the coupling length and maximum power transfer efficiency. From the graph in Fig. 3.11, we determine when the value of thickness or refractive index meets the phase matching requirement, and also when the coupling length reaches a 
maximum. The incident wavelength is $633 \mathrm{~nm}$ (He-Ne laser).
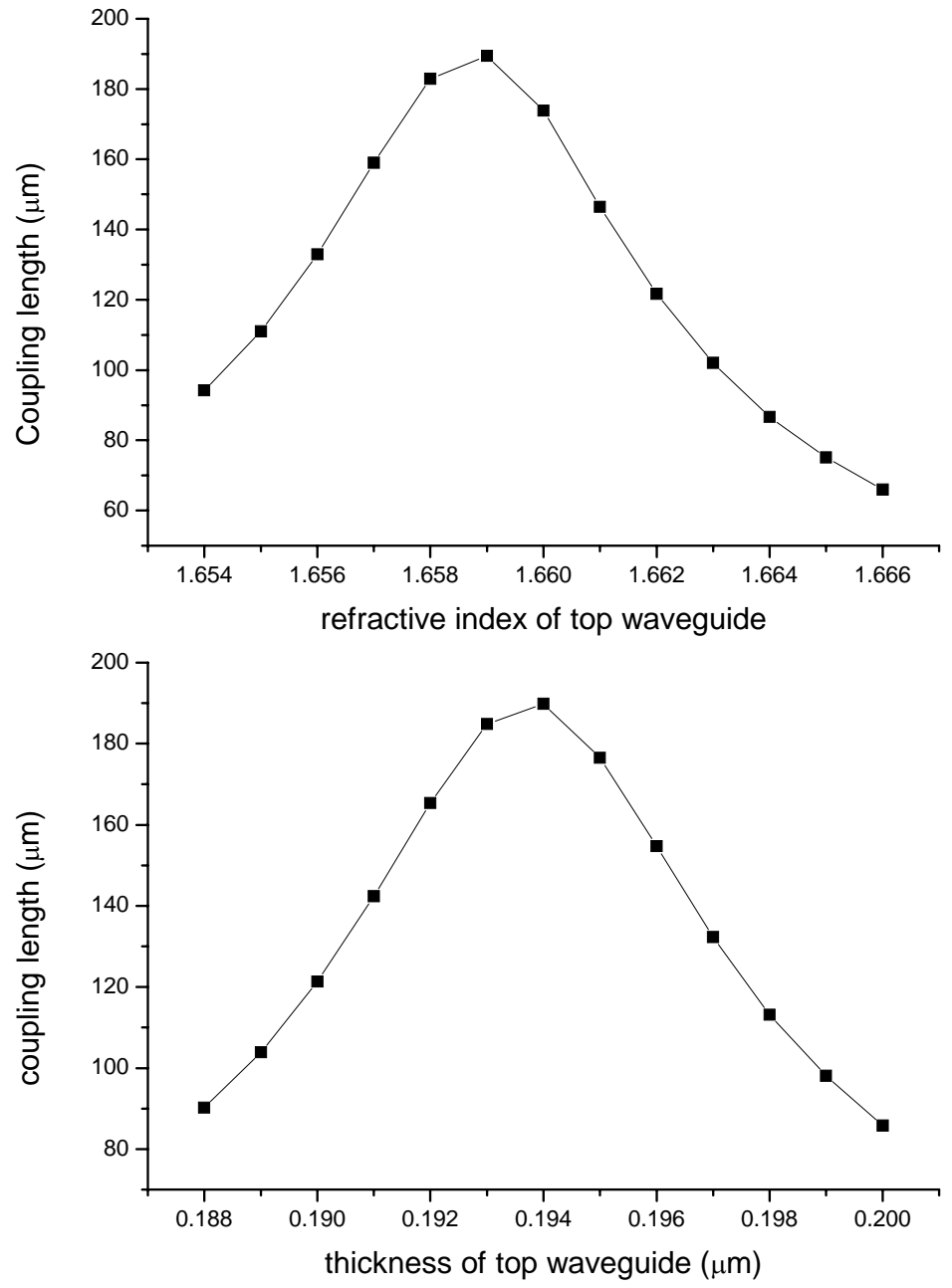

Fig 3.11. Coupling length vs. thickness and refractive index at $633 \mathrm{~nm}$.

\section{3-4 Biolayer influence for light propagation in coupled waveguide}

Based on the coupled waveguides, we can proceed further with a simulation for the whole device system. A simple SPARROW structure is shown in Fig. 3.12. Compared to the bottom waveguide, the top biolayer will affect the phase matching requirement greatly. To get the phase matching, we have to get the top waveguides' 
effective refractive index first. Now, there are four layers for the top waveguide, as shown in Fig 3.13, while the bottom waveguide remains the same.

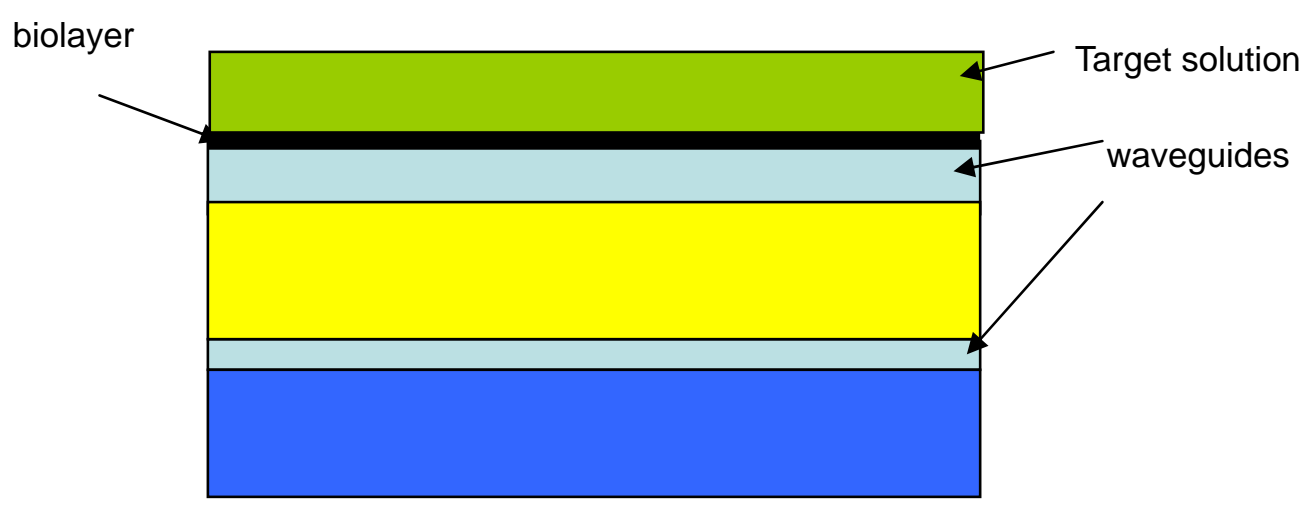

Fig. 3-12. Simple basic structure of SPARROW biosensor.

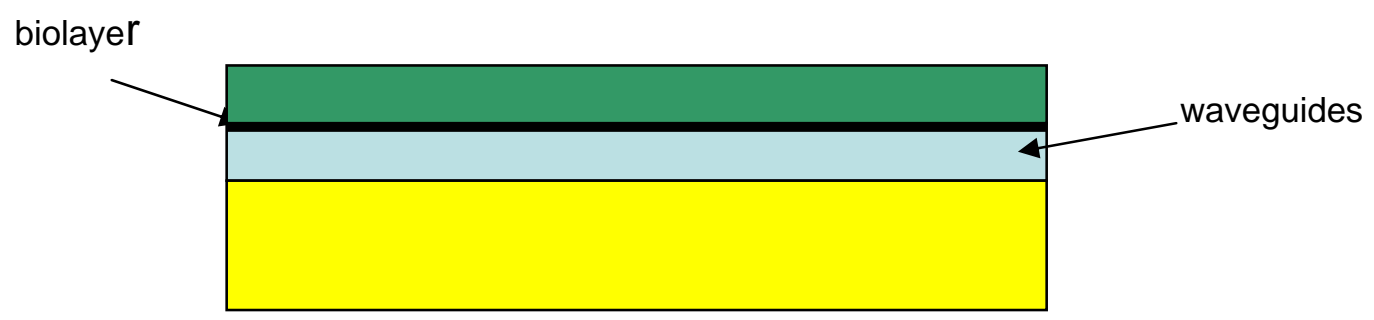

Fig 3.13. Top waveguide structure.

It is necessary to get the effective refractive index and propagation constant of this waveguide with the biolayer. Assuming the biolayer is homogenous and the thickness of the biolayer is $30 \mathrm{~nm}$, the refractive index of biolayer is 1.45 . We can get a new mode and new effective refractive index in this waveguide. Figure 3.14 shows the mode shape comparison with and without the biolayer. Also, the effective refractive without biolayer is 1.5098 and with biolayer will be 1.5144. From Fig. 3.14 and value of effective refractive indices, there is not too much difference between the two mode shapes. Next, we will see how this small change in refractive index affects the light coupling in the waveguides. 


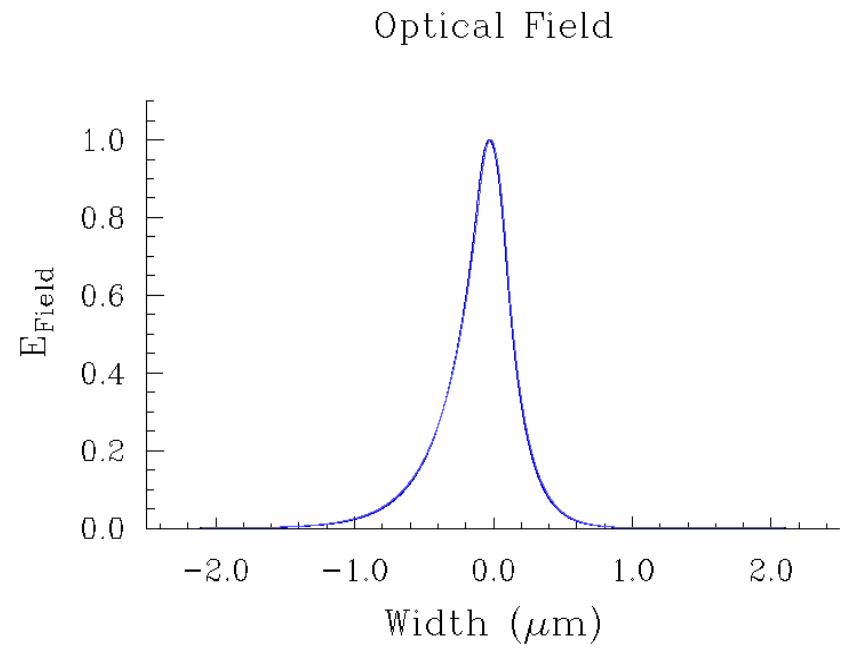

Fig. 3-14 Mode shape comparison with and without the biolayer.

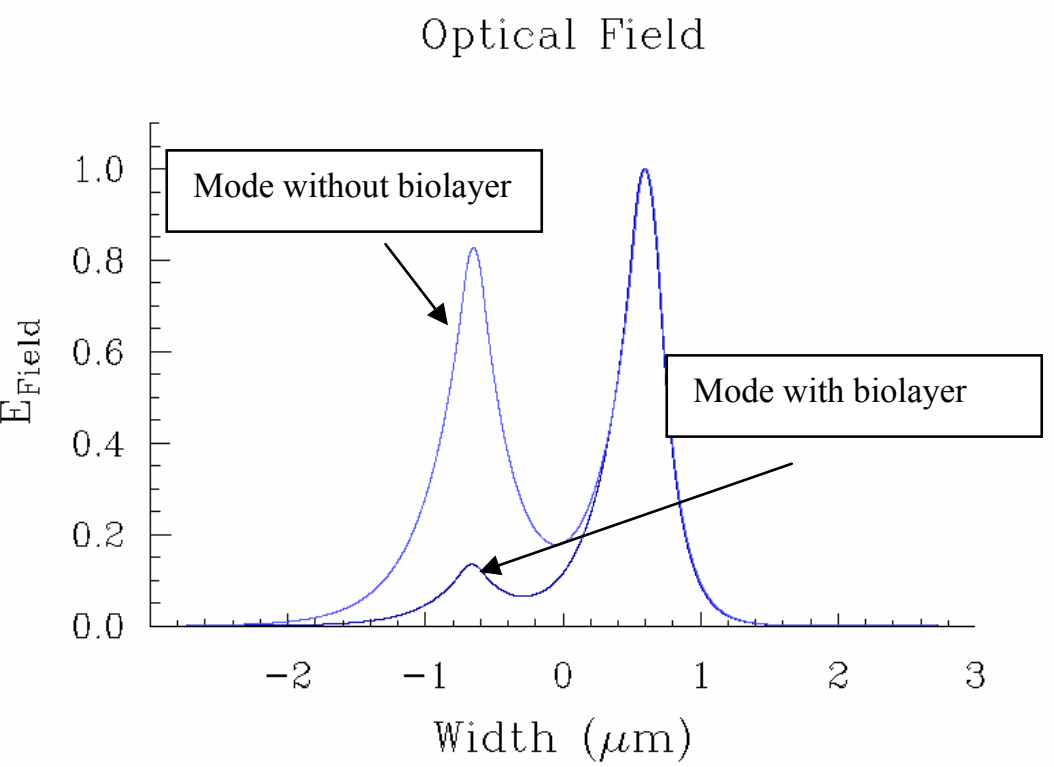

Fig 3.15 Mode comparison in coupled waveguide with and without a biolayer.

From Fig. 3.15, the modes have totally different shapes in coupled waveguides with and without a biolayer. Also, the computer simulation gives different results when a biolayer exists (see Fig. 3.16). 

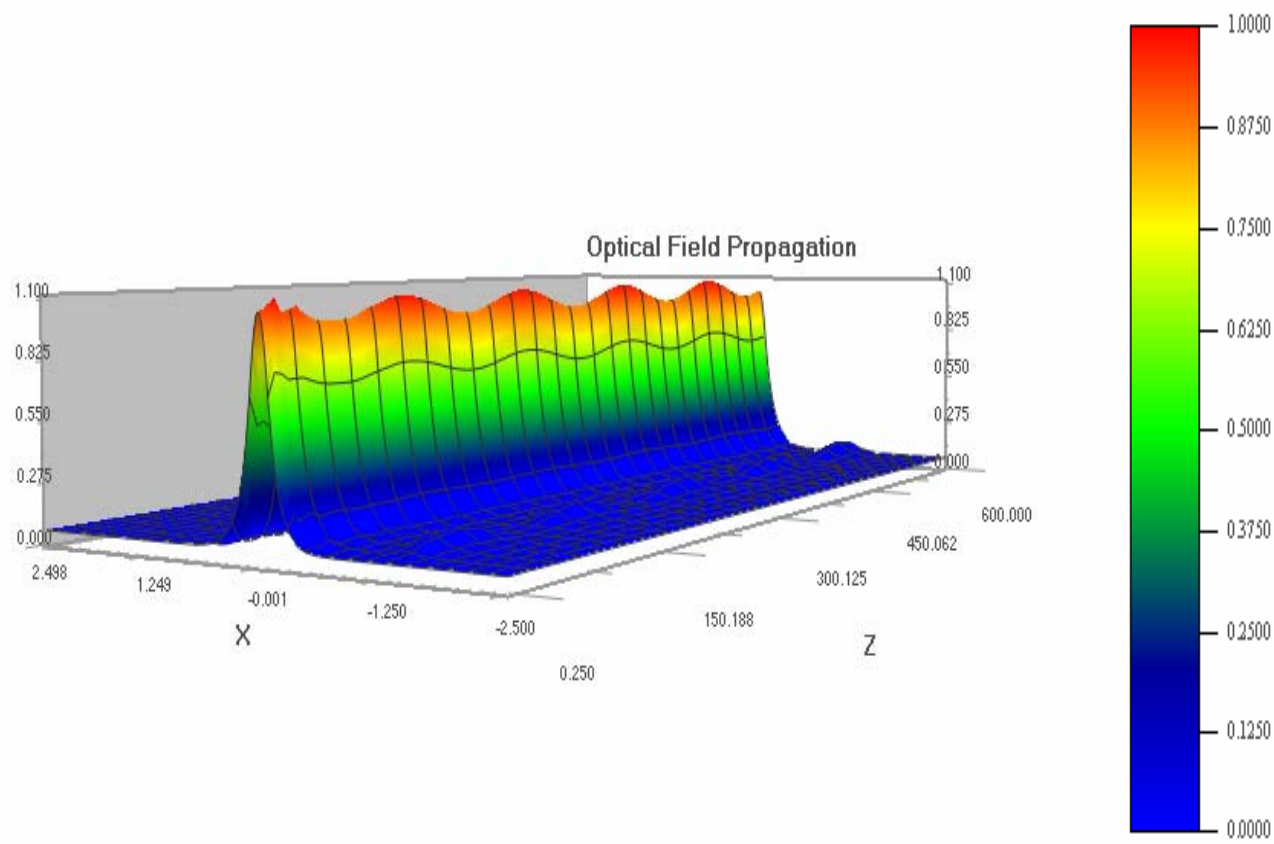

Fig. 3.16. Light intensity in coupled waveguides with biolayer (almost no coupling).

We require phase matching between buried waveguide and biolayer coated waveguide. So, the thickness of the top waveguide has to be designed again. In this case, only if the thickness of the top waveguide is equal to $185 \mathrm{~nm}$, the computer simulation shows that the light intensity is completely coupled in these two waveguides. The coupling length is $191 \mu \mathrm{m}$. This is illustrated in Fig. 3.17. However, if the top waveguide is not fully covered by biolayer, detuning factor will be large. When the percentage coverage drops to zero, the coupling length is only $63 \mu \mathrm{m}$ and the coupling efficiency is close to zero (see Fig. 3.18). 
Optical Field

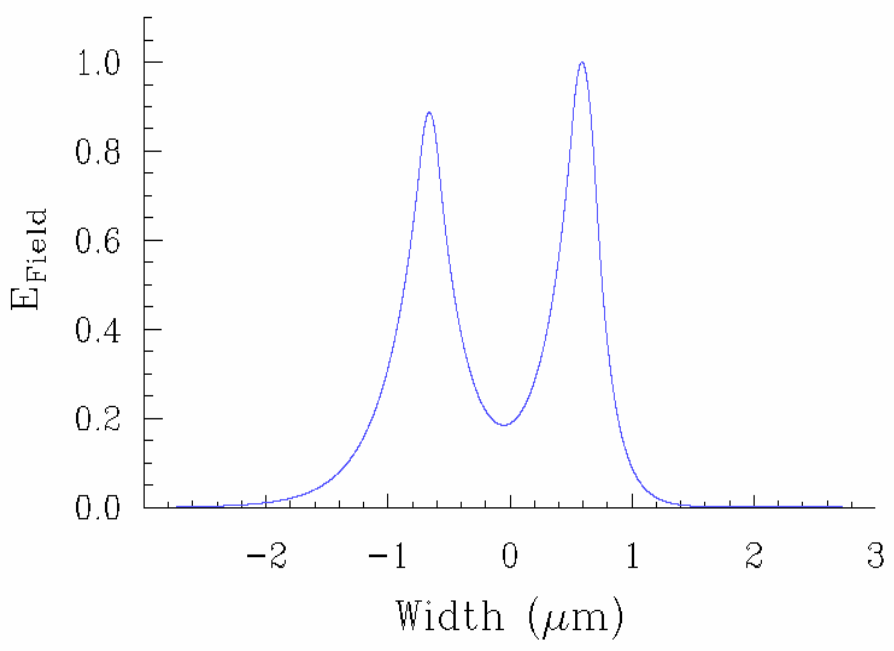

(a)

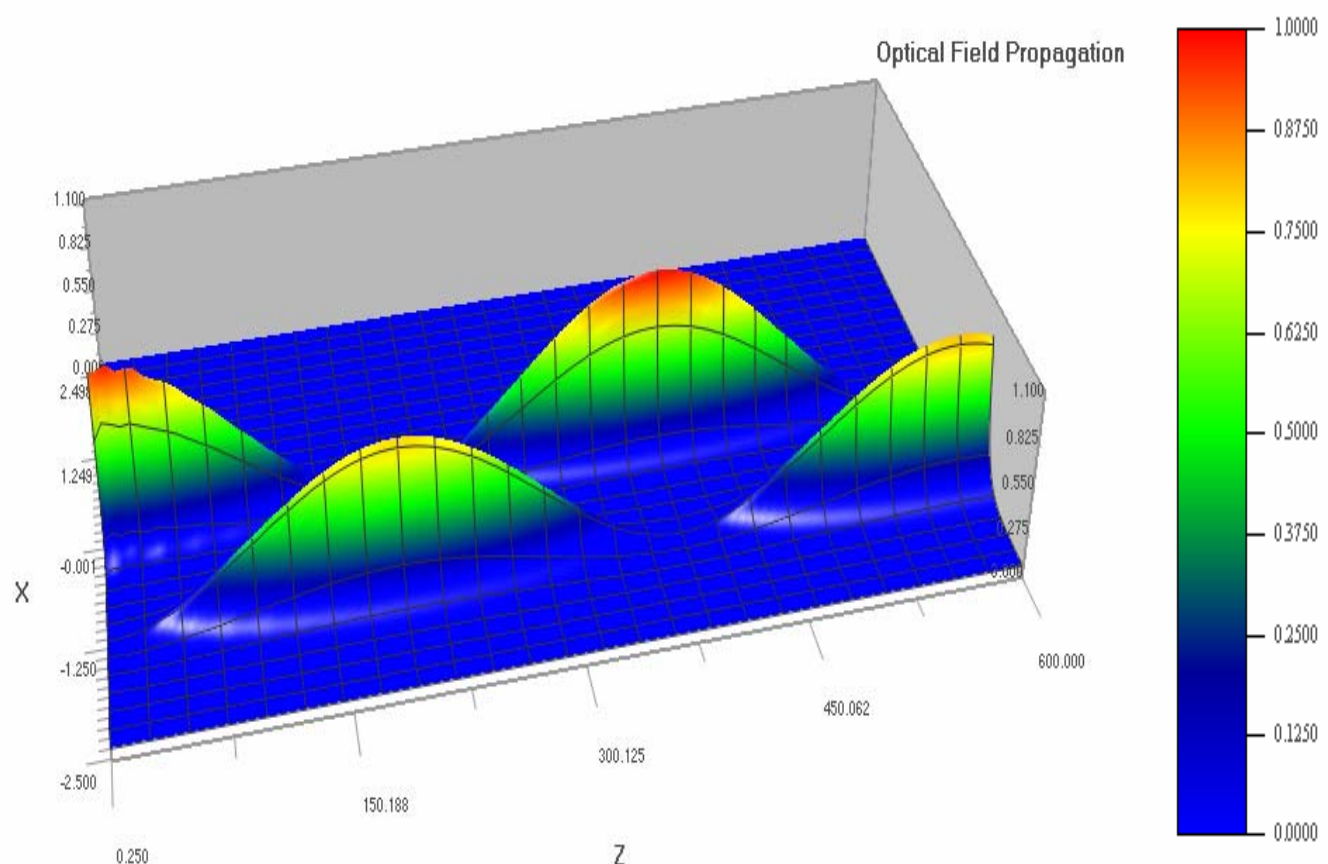

(b)

Fig. 3.17. (a) Mode in coupled waveguide with biolayer. (b) Light intensity in coupled waveguides with a biolayer (coupling length $191 \mu \mathrm{m}$ ). 


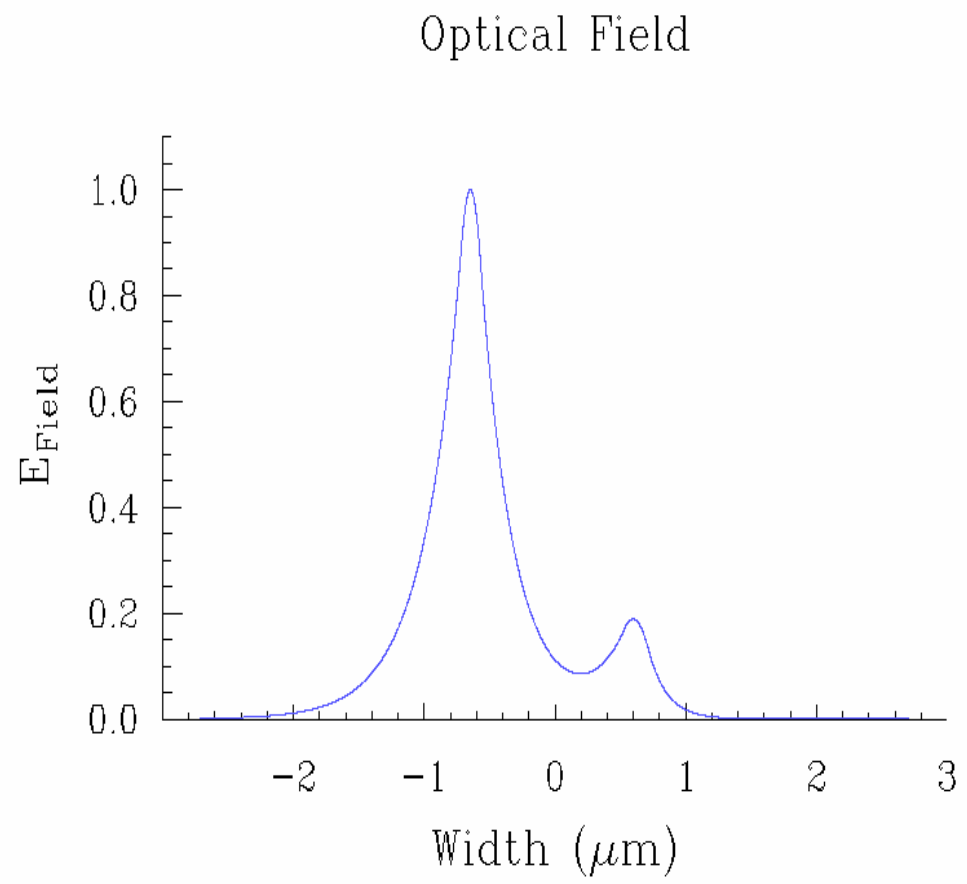

(a)
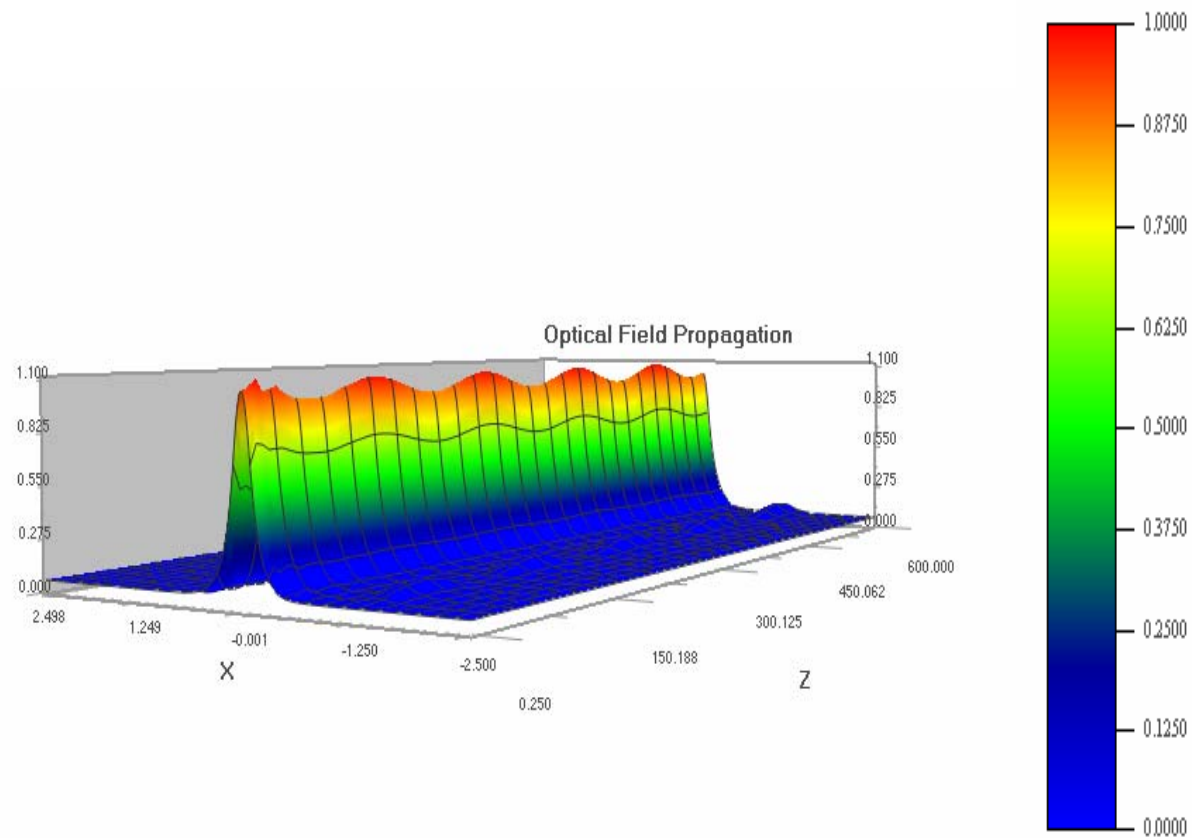

(b)

Fig. 3.18. (a) Mode in coupled waveguide without biolayer. (b) Light intensity in coupled waveguides without a biolayer (coupling length of $63 \mu \mathrm{m}$ ). 


\section{3-5 Mathematical model for ideal structure-numerical results}

According to coupled mode theory, there are two ways we can get numerical results for coupled waveguides - conventional coupled mode theory (or orthogonal coupled mode theory), and non-orthogonal coupled mode theory (Haus et al., 1991; Huang, et al., 1994). In this section, we will see the difference in the numerical results and compare the results to computer simulations. These calculations have been done in 'Mathematica', a scripting language, and the calculation program is provided in Appendix I.

For an ideal directional coupler structure without a biolayer, the OPTIBPM simulation program gives the coupling length to be $186 \mu \mathrm{m}$ and maximum power transfer efficiency is $100 \%$. Following conventional coupled mode theory, the coupling length is $172 \mu \mathrm{m}$ and power transfer efficiency is $100 \%$. Non-orthogonal coupled mode theory gives coupling length to be $187 \mu \mathrm{m}$ and power transfer efficiency of 100\%. Figure 3.19 shows the results of these three methods. The dashed line is the result of conventional coupled mode theory. The OPTIBPM simulation results agree with non-orthogonal coupled mode theory, which is more accurate than conventional coupled mode theory.

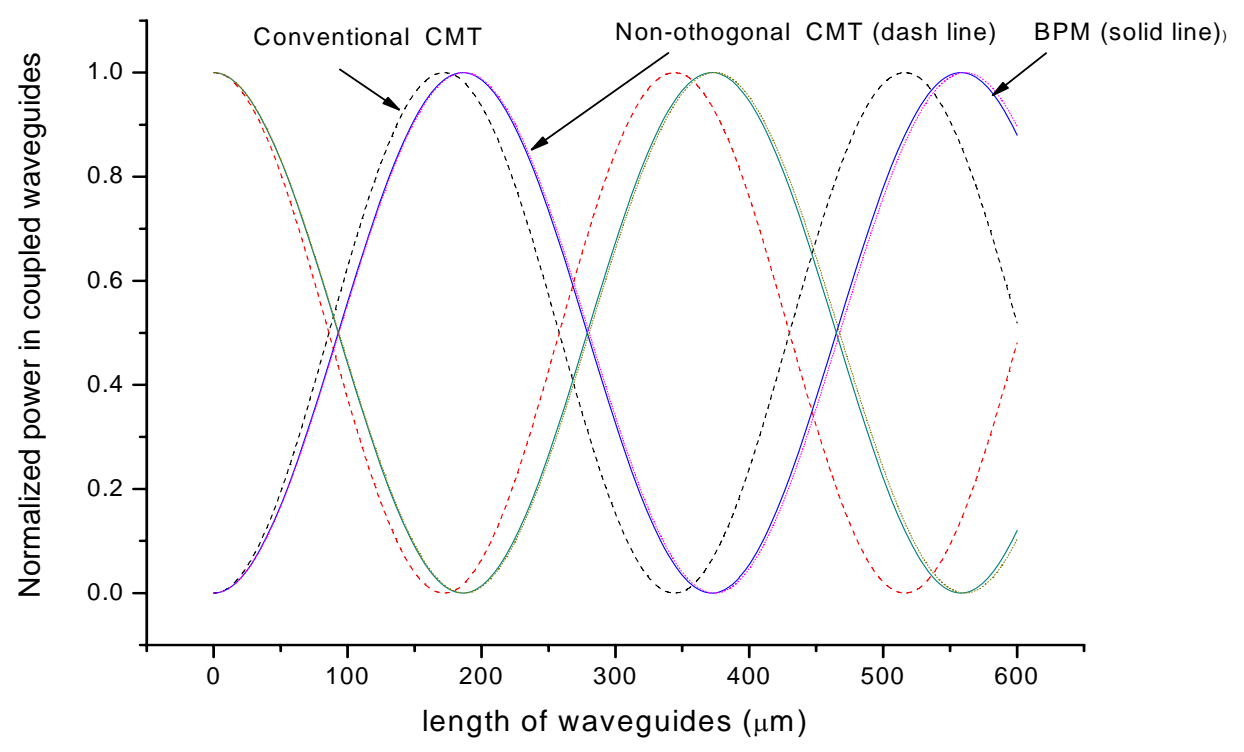

Fig. 3.19. Comparison of numerical results obtained using three methods (see text for discussion). 
When the effect of a biolayer is considered, the effective refractive index of the top waveguide changes. Using the substrate index $\boldsymbol{n}_{\text {substrate }}$, the waveguide index $\boldsymbol{n}_{\text {waveguide }}$, the thickness d, biolayer index $\boldsymbol{n}_{\text {biolayer }}$, biolayer thickness s, and cladding index $\boldsymbol{n}_{\text {cladding }}$, we have the new effective refractive index equation:

$$
N_{\text {eff }}=n_{\text {eff } 1}+\left(n_{\text {eff } 2}-n_{\text {eff } 1}\right) e^{-\gamma s}
$$

Here, $\boldsymbol{n}_{\text {eff } 1}$ is the effective index of top layer without biolayer and $\boldsymbol{n}_{\text {eff }}$ is the effective index of top layer without cladding. $\gamma$ is the parameter which can be obtained from simulation model (See Fig. 3.20). For different structure, we will have different $\gamma$. Thus, the propagation constant of the top waveguide can be calculated from which we can get the coupling length and maximum power transfer efficiency in coupled waveguides. Figure 3.21 shows the numerical results of the three methods discussed assuming an ideal structure with biolayer $\left(\boldsymbol{n}_{\text {biolayer }}=1.4\right)$.

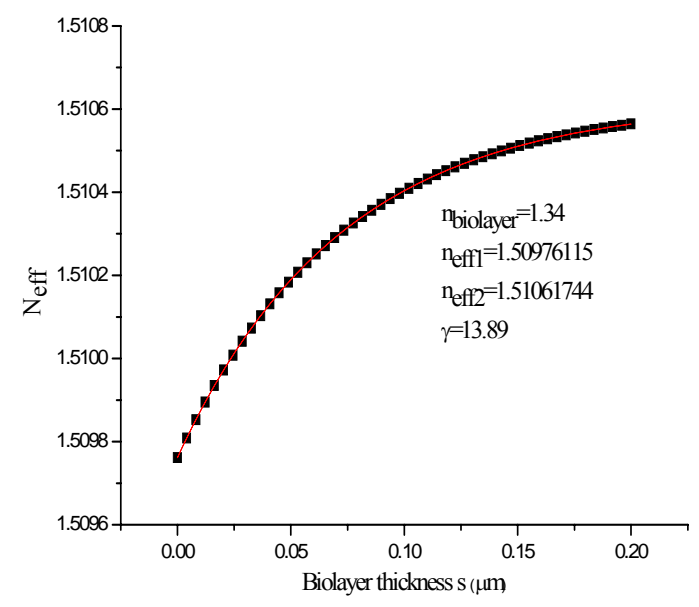

(a)

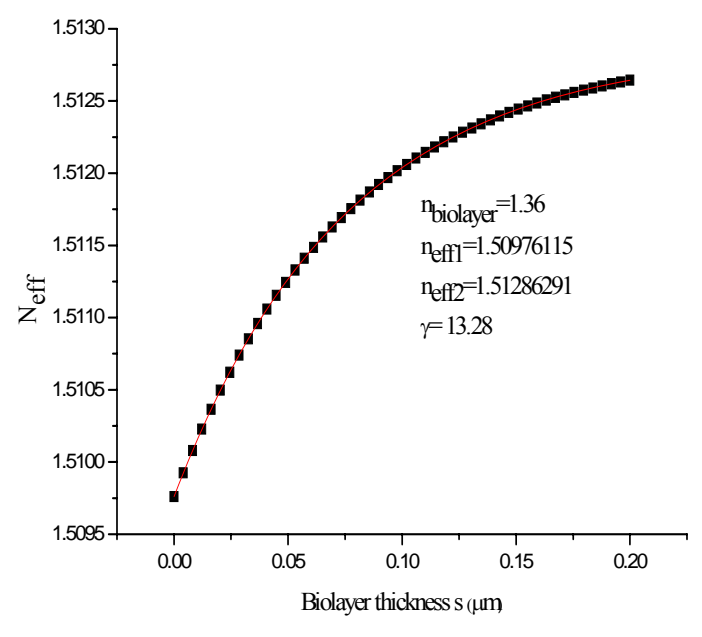

(b)

Fig. 3.20 The fitting $\gamma$ value with different biolayer indices

(a) The $\gamma$ value with biolayer index 1.34 (b) The $\gamma$ value with biolayer index 1.36 Dots: Simulation data; Line: Fitting data 


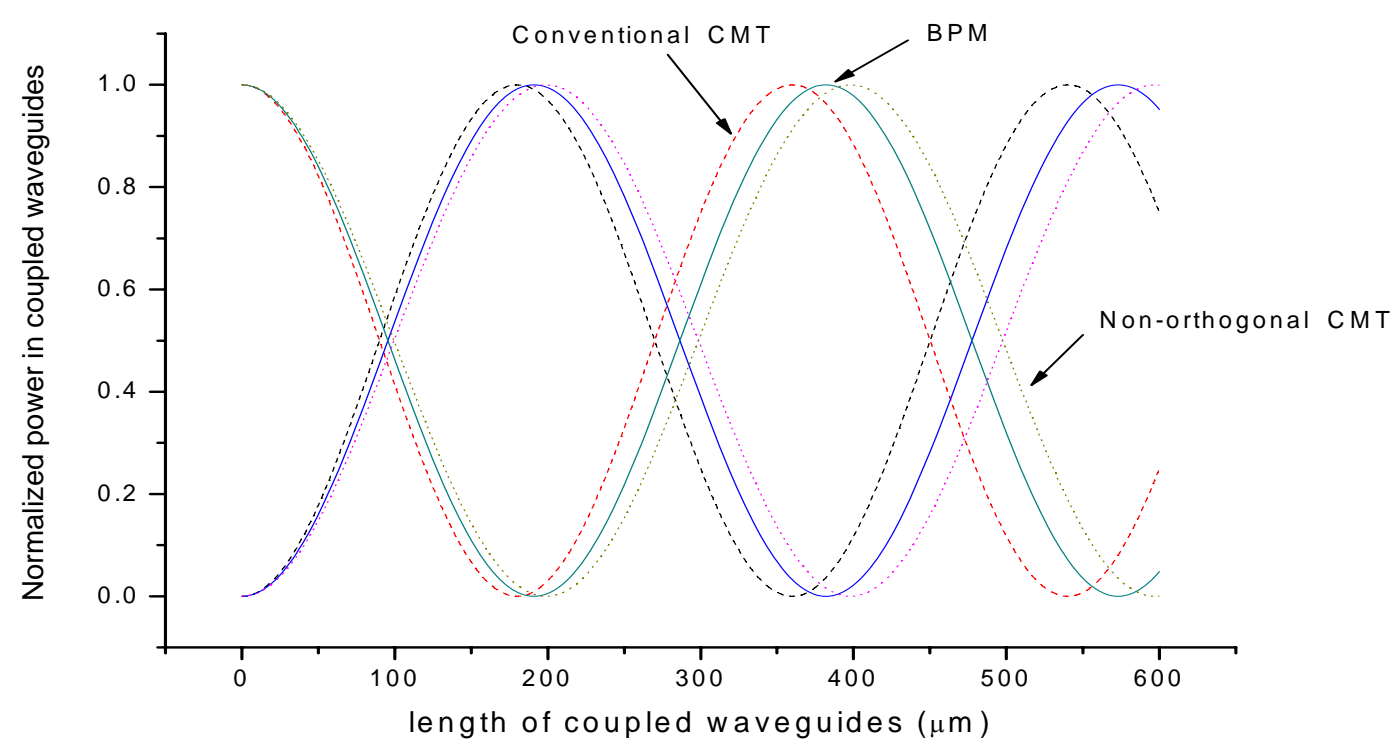

Fig. 3.21. Different numerical results by three methods. (see text for discussion) Coupling length: a) Conventional CMT, $180 \mu \mathrm{m}$ b) Non-orthogonal, $199 \mu \mathrm{m}$ c) $\mathrm{BPM}, 191 \mu \mathrm{m}$ 


\section{Chapter 4}

\section{Biolayer Construction}

In order to model the SPARROW biosensor, certain properties of both the transducer and biolayer need to be known. These properties include the refractive index, thickness, and roughness values, which can be obtained using experimental methods. However, these properties often vary significantly from sample-to-sample, and for the same sample, different experimental methods can give different results. To help define the appropriate range of parameters needed for the modeling tasks, a series of experimental measurements were undertaken. This chapter describes experimental techniques that were used to measure the samples studied during this dissertation work. Section 4-1 describes biolayer design, which was developed with Dr. Minsoo Lim (WVU Chemistry Department). Section 4-2 describes measurement techniques used to characterize samples, and section 4-3 will describe the experimental results.

\section{4-1 Biolayer design}

The physical properties of the biolayer play an important role in biosensor operation. For the SPARROW biosensor, there are several requirements which the biolayer must meet. The first requirement is the biolayer thickness. To achieve good sensitivity, there should be sufficient power reaching the biolayer surface. Therefore, the biolayer thickness should be less than the penetration depth, which is defined as the distance over which the power in the waveguide drops to 1/e. Second, the biolayer should recognize the binding events of specific molecules. Third, the biolayer should be capable of regeneration, in order to be an economically feasible device. When designing the biolayer, these three requirements present a big challenge. Several different kinds of structures were tested during the course of this work. One of these structures is shown schematically in Fig. 4.1.

The first step to successfully grow a biolayer is to find an appropriate medium to connect organic and non-organic materials. Self-assembled monolayers (SAMs) having thickness of about $2 \mathrm{~nm}$ were chosen here to coat a bare alumina surface. The second 


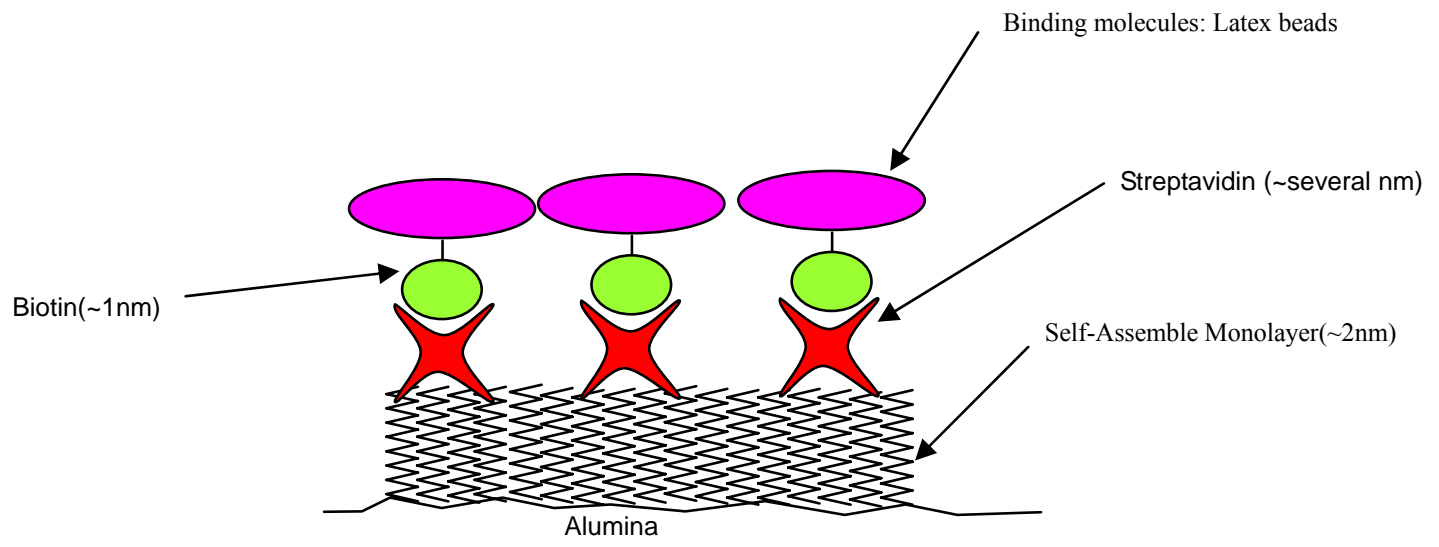

Fig. 4.1. Construction Scheme of Biolayer.

step is to link a streptavidin layer (of several $\mathrm{nm}$ in thickness) to the SAMs. The thickness of streptavidin (several $\mathrm{nm}$ ) and its binding to biotin make it an attractive choice for our biolayer (discussed in Section 4-3-3). Generally, the size of biotin is less than $1 \mathrm{~nm}$, but it will offer strong binding to both the streptavidin and the specific molecules which we want to detect. A functional test was performed by using latex microbeads which simulate anthrax spores. By flowing a medium containing microbeads across the biolayer, we found that some microbeads chemically bind to biolayer. The percent coverage of these binding molecules then determined by how much the output power of the biosensor would change. This will be described in Section 4-3-4.

\section{4-2 Analytical tools for organic film measurements}

In studies of organic films, we are interested in both their surface conditions and optical properties, such as refractive indices. The analytical tools to obtain this information have been dramatically developed in last decade (Ulman, 1991). There are four different tools I used in my dissertation work-(1) ellipsometry measurements, (2) atomic force microscopy (AFM), (3) contact-angle measurements, and (4) fouriertransform infrared (FTIR) spectroscopy. A brief introduction and explanation of the physics principles for these tools are given in the following sections. Some of the experimental results are then shown in Section 4-3. Also, images taken using a 
fluorescence microscope are included in this chapter. These images helped determine the micro-bead coverage on our layers.

\section{4-2-1 Ellipsometry}

Ellipsometry is a technique which can provide film thickness and refractive index information. This technique uses the principle that reflection at a dielectric interface depends on the polarization of the incident light, while the transmission of light through a transparent layer changes the phase of the incoming wave depending on the refractive index of the material. An ellipsometer can measure layer thicknesses as small as $1 \mathrm{~nm}$ up to layers which are several microns thick. The ability to measure small thicknesses makes this technique especially useful for characterization of biolayers.

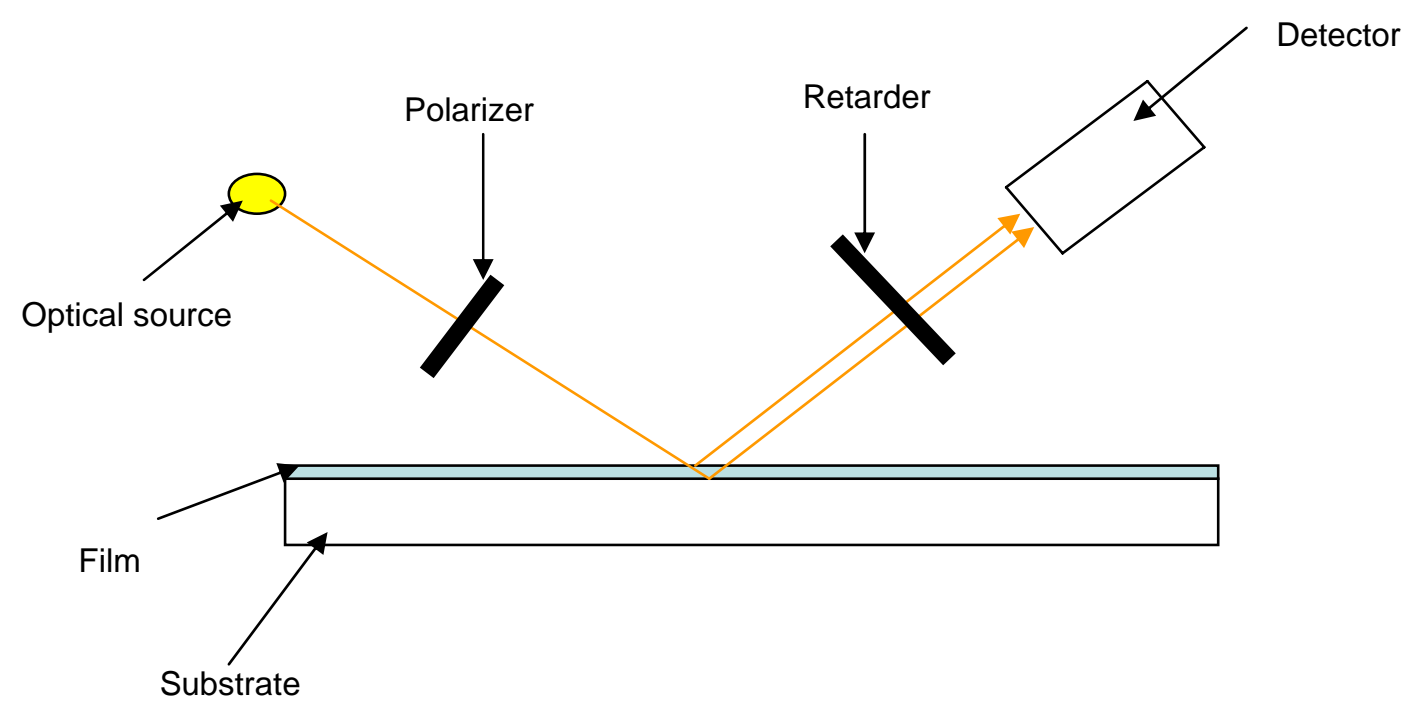

Fig 4.2. Principle of operation for an ellipsometer.

A typical ellipsometer set-up is shown in Fig. 4.2. Incident light passes through a linear polarizer and a quarter-wave plate. The polarization of the incident light can be varied from linearly polarized to elliptically polarized to circularly polarized by adjusting the angle between the transmission axis of the linear polarizer and the fast axis of the quarter-wave plate. The reflected beam from the sample passes through a retarder before 
entering the detector. This retarder provides an optical retardation between the two incoming polarizations which exactly compensates for the optical retardation caused by the polarization dependent reflections at each dielectric interface. A detector is used to receive the s- and p- polarized light and measure the ratio of their reflection coefficients. In Ellipsometer data analysis software, the measured values are expressed as psi $(\psi)$ and delta $(\Delta)$, Eq. 4-1 gives the relationship between these two parameters and reflection coefficients.

$$
\rho=\frac{R_{p}}{R_{s}}=\tan (\psi) e^{i \Delta}
$$

where $R_{p}$ and $R_{s}$ are Fresnel reflection coefficients for p- and s- polarized light. Fig 4.3 shows how ellipsometer data analysis software provides the film information such as complex refractive index, thickness and roughness after ellipsometry measurement.

\section{Measurement (Experimental data)}

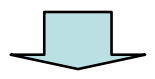

\section{Build a Model (generate data)}

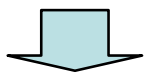

Fit Parameters

(Compare with experimental data)

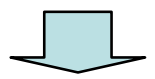

\section{Results}

Improve model

Fig. 4.3 Basic procedure used in ellipsometry measurements determine materials properties from experimental data. 
The ellipsometer used in this work is shown in Fig. 4.4. This ellipsometer is ideal for a large variety of applications, including measurements of dielectrics, polymers, semiconductors, metals, etc. The white-light source is the combination of Quartz Tungsten Halogen (QTH) lamp and Deuterium $\left(\mathrm{D}_{2}\right)$ lamp, which have spectra range from UV to infrared. On the sample surface, the light beam covers a spot size of approximately $1 \sim 2 \mathrm{~mm}$ in radius. The detected light beam is dispersed and 470 wavelengths from $245 \mathrm{~nm}$ to $1000 \mathrm{~nm}$ (NIR extension to $1700 \mathrm{~nm}$, need use InGaAs detector replace silicon array) are simultaneously measured by a charge-coupled device (CCD) detector array. A broad range of film thicknesses (sub-nanometer to 10 microns) are accessible.

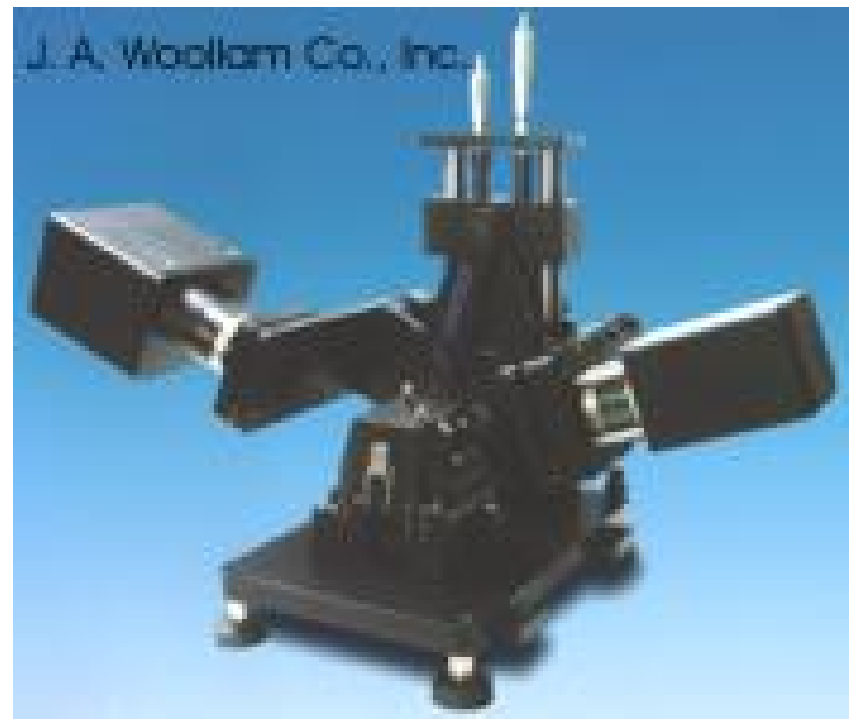

Fig. 4.4. M2000U ellipsometer from J. A. Woolam Co.

(located in Lane Department of Computer Science and Electrical Engineering).

\section{4-2-2 Atomic force microscopy (AFM)}

Another analytical tool used in this work is the atomic force microscope (AFM). From AFM images, we obtained surface information for both the bare waveguides and for the biolayers that were subsequently deposited. The AFM is one of about two dozen types of scanned-proximity probe microscopes. Typically, these microscopes work by measuring a local property, such as height, optical absorption, or magnetism, with a probe or "tip" placed very close to the sample. The small probe-sample separation, which 
depends on the order of the instrument's resolution, makes it possible to take measurements over a small area. To acquire an image, the microscope raster-scans the probe over the sample surface while measuring the local property in question. The resulting image resembles an image on a television screen in that both consist of many rows or lines of information placed one above the other. The AFM operates by measuring attractive or repulsive forces between a tip and the sample (Binnig et al., 1986).

Figure 4.5 illustrates how an AFM works. As the cantilever flexes, the light from a laser is reflected onto the split photo-diode. By measuring the difference signal (A-B), changes in the bending of the cantilever can be measured. Since the cantilever obeys

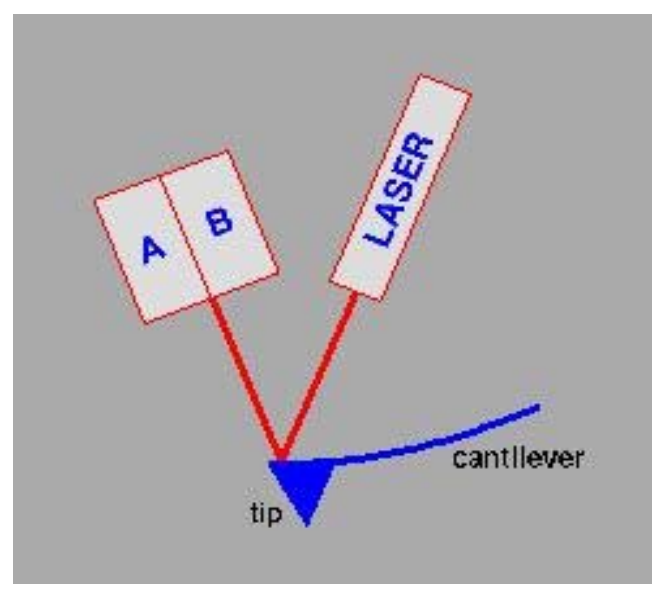

Fig. 4.5. Principle of general AFM operation.

Hooke's Law for small displacements, the interaction force between the tip and the sample can be found. The movement of the tip or sample is performed by an extremely precise positioning device made from piezo-electric ceramics, most often in the form of a tube scanner. The scanner is capable of sub-angstrom resolution in the $\mathrm{x}-, \mathrm{y}-$ and $\mathrm{z}-$ directions. The z-axis is conventionally chosen perpendicular to the sample. In principle, AFM is like a recorder. It can achieve a resolution of $10 \mathrm{pm}$ and, unlike electron microscopes, can image samples in air and under liquids. Following are the general properties of an AFM: sensitive detection, flexible cantilevers, sharp tips, high-resolution tip-sample positioning, and force feedback. 
The three main classes of interaction by which AFM image contrast is obtained are contact mode, tapping mode and non-contact mode. The contact mode is the most common method of operation of the AFM. As the name suggests, the tip and sample remain in close contact as the scanning proceeds. In non-contact mode, the AFM derives topographic images from measurements of attractive forces and the tip does not touch the sample. It is a very difficult mode to operate in ambient conditions because the thin layer of water contamination which exists on the biolayer surface on the sample will invariably form a small capillary bridge between the tip and the sample and cause the tip to jump-tocontact. When imaging soft samples such as a biolayer, the tapping mode is a far better choice than other two methods. The cantilever is oscillated at its resonant frequency (often hundreds of kilohertz) and positioned above the surface so that it only taps the surface for a very small fraction of its oscillation period (James, et al., 2001). Moreover, there has been much interest in phase imaging. This works by measuring the phase difference between the oscillations of the cantilever driving piezo and the detected oscillations (Magonov, 1997). The tapping mode was used in this work. In our experiments, we used the Digital Instruments multimode AFM shown in Fig. 4.6 (located in the WVU Physics Department). The images shown in this dissertation were provided by Dr. Minsoo Lim, of the WVU Chemistry Department.

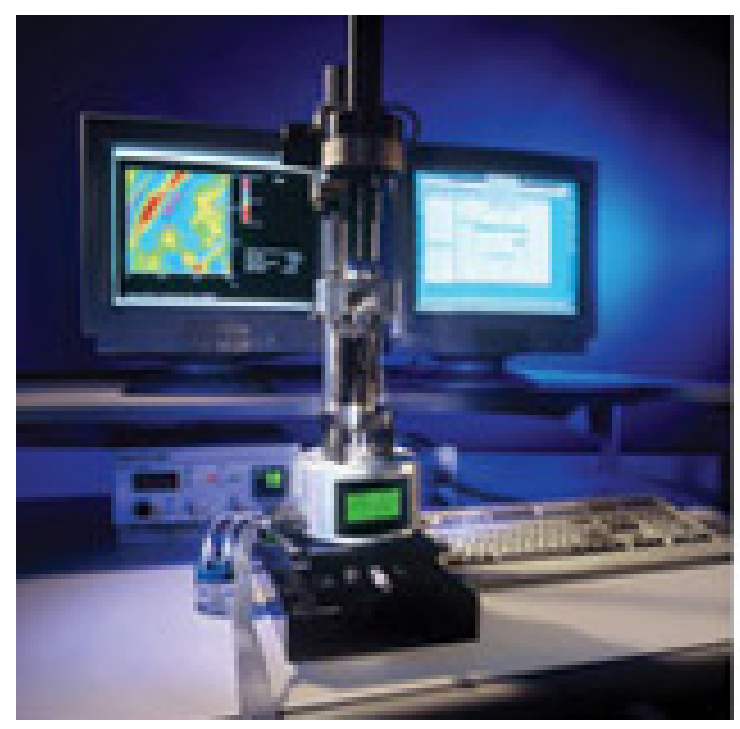

Fig. 4.6. The Digital Instruments multimode AFM (in the WVU Physics Dept.). 


\section{4-2-3 Contact-angle measurement}

Surface coverages of a stable monolayer and multilayer film can be estimated from wetting measurements. The reason is that the shape of a liquid drop on the material's surface is affected by the free energy of this surface. We call this experiment a contact-angle measurement. Either water or hexadecane, or some other solution, is dropped to the surface of the sample and the angle between the tangent line and the solid surface can be measured. This angle is called the contact angle, and is shown schematically in Fig. 4.6. If a liquid with well-known properties is used, the resulting interfacial tension can be used to identify the nature of the solid film. This technique is extremely surface sensitive, with the ability to detect properties of a monolayer (Shieh, 2001).
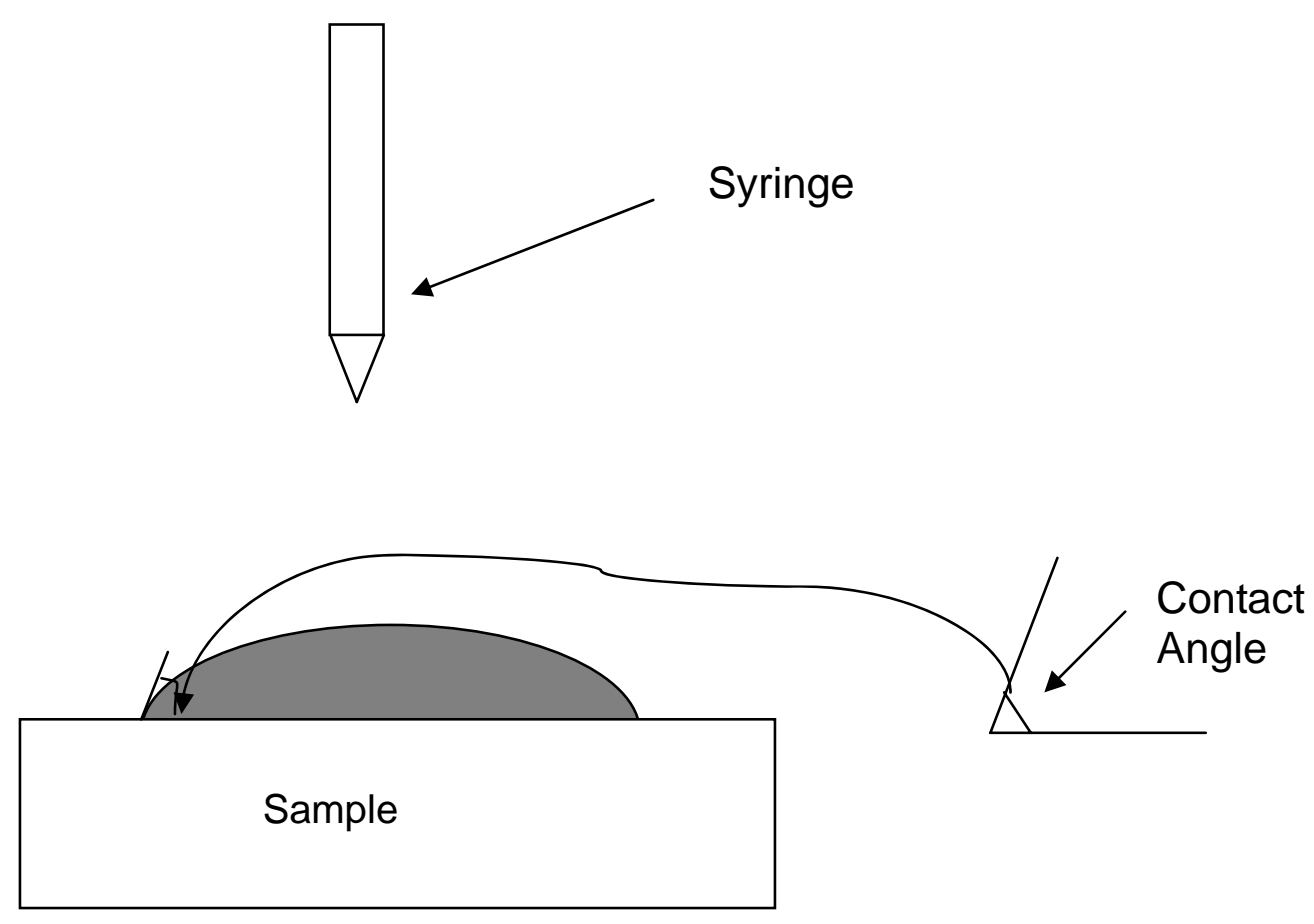

Fig. 4.7. Principle of contact-angle measurement.

As shown in Fig. 4.7, when a droplet of liquid rests on the surface of a solid, the shape of the droplet is determined by the balance of the interfacial liquid/vapor/solid forces. Moreover, if an organic film exhibits hydrophobic behavior and the bare sample surface, such as alumina or sapphire used in the biosensor transducer, exhibits 
hydrophilic behavior, the contact angle will be proportional to the percent coverage of the organic film. Thus, the measured contact angle can be used to roughly determine the percent coverage of organic film.

Figure 4.8 shows the device model FTA125 (First Ten Angstroms Co.) used in this work. It is a general purpose model appropriate for smaller specimens. It can measure both contact angles and surface tensions. The specimen is placed on a stage and raised to an appropriate level by the rack-and-pinion lift. The specimen can be quite large: up to $250 \mathrm{~mm}$ in width and $25 \mathrm{~mm}$ in thickness. Even though the camera is oriented vertically, the optical axis is horizontal because of a $90^{\circ}$ turning mirror; the drop is viewed from the side. This instrument is located in the WVU Chemistry Department.

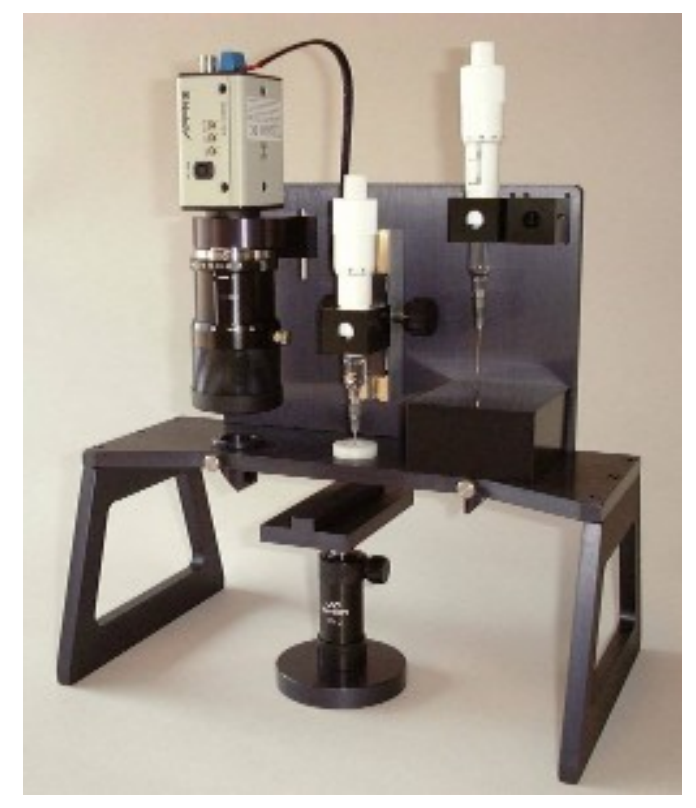

Fig. 4.8. FTA125 for contact angle measurement (WVU Chemistry Dept).

\section{4-2-4 Fourier-transform infrared (FTIR) spectrometer}

One of the most useful equipment systems to measure absorption data in the infrared is the fourier-transform infrared (FTIR) spectrometer. In infrared spectroscopy, IR radiation is passed through a sample. Ignoring reflective or scattering losses, some of 
the infrared radiation is absorbed by the sample and the remainder is passed through (i.e., transmitted). The resulting light-intensity spectrum represents the molecular absorption "fingerprint" of the sample. Like a fingerprint, no two unique molecular structures produce the same infrared spectrum.

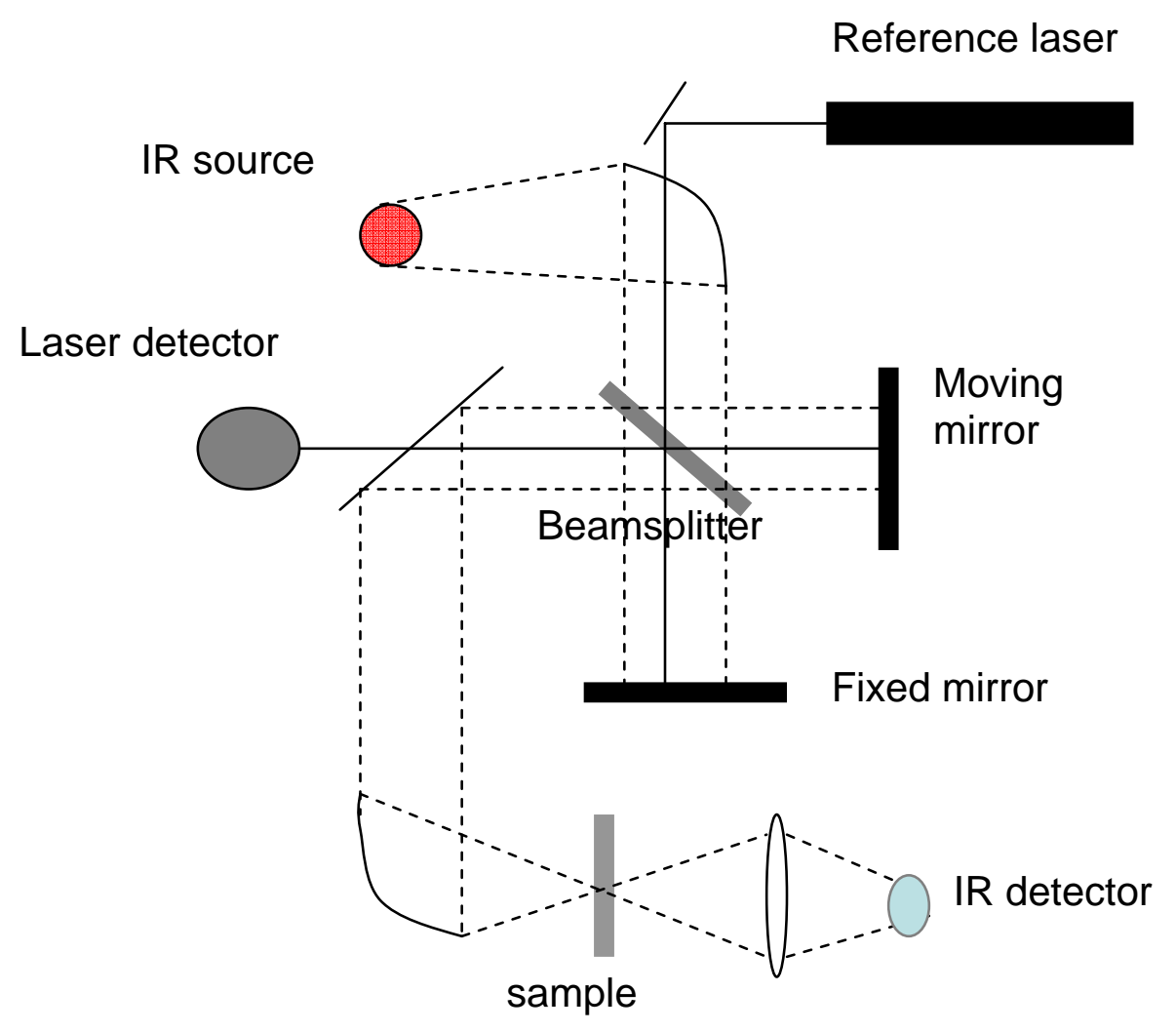

Fig. 4.9. Schematic of an FTIR.

An FTIR (Fig. 4.9) contains an interferometer, which is made up of a fixed mirror, a moving mirror, and a beamsplitter. The interferometer produces a unique type of signal which has all of the infrared frequencies "encoded" into it. The signal can be measured very quickly, usually on the order of one second or so. Thus, the time element per sample is reduced to a matter of a few seconds rather than several minutes. The beamsplitter divides the incoming infrared beam into two optical beams. One beam reflects off of a flat mirror which is fixed in place. The other beam reflects off of a flat mirror which is on a mechanism which allows this mirror to move a very short distance (typically a few millimeters) away from the beamsplitter. The two beams reflect off of 
their respective mirrors and are recombined when they meet back at the beamsplitter. Because the path that one beam travels is a fixed length and the other is constantly changing as its mirror moves, the signal which exits the interferometer is the result of these two beams "interfering" with each other. The resulting signal is called an interferogram which has the unique property that every data point (a function of the moving mirror position) which makes up the signal has information about every infrared frequency which comes from the source. This means that as the interferogram is measured, all frequencies are being measured simultaneously. Thus, the use of the interferometer results in extremely fast measurements. Because the user typically requires a frequency spectrum (a plot of the intensity at each individual frequency) in order to make sample identification, the measured interferogram signal is not interpreted directly. A means of "decoding" the individual frequencies is accomplished via a wellknown mathematical technique called the Fourier transformation. This transformation is performed by the computer which then presents the user with the desired spectral information for analysis.

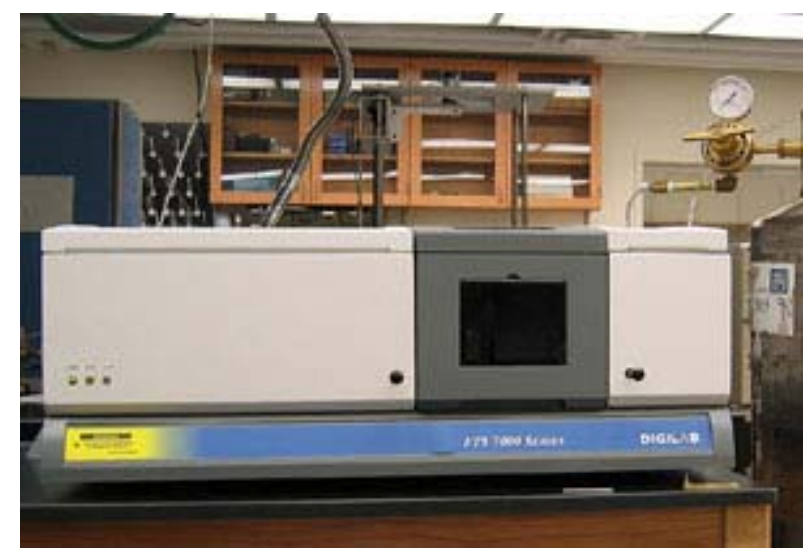

Fig. 4.10. Digilab FTS 7000 spectrometer (located in WVU Lane Dept. of Computer Science and Electrical Engineering).

In our experiment, a Digilab model FTS 7000 spectrometer was used. A picture of the instrument is shown in Fig. 4.10. Samples were measured at room temperature using a Mercury Cadmium Telluride (MCT) detector and a $\mathrm{KBr}$ beamsplitter. To get absorption peaks of SAM, both a bare sample and a SAM coated sample were scanned. Set spectrum of a bare sample $\left(I_{0}\right)$ as the background and take the ratio, both 
transmittance $\left(T=I / I_{0}\right)$ and absorbance spectrum $(A=-\log (T))$ can be obtained. Nitrogen gas is purged to chamber to reduce the noise. And the parabolic mirror is used to focus the IR beam on the surface of the sample (spot size is about half millimeter). The FTIR data were used to detect the distinct absorption peaks of the biolayer and thus to prove its existence.

\section{4-3 Experimental results and analysis}

\section{4-3-1 Bare sapphire and alumina}

To construct a biolayer, a well prepared substrate is needed. Both alumina and sapphire wafers were investigated in this work. Alumina is a cost effective and widely used material in the family of engineering ceramics. The raw materials from which this high performance technical grade ceramic is made are readily available and reasonably priced, resulting in good value for the cost in fabricated alumina shapes. With an excellent combination of properties and an attractive price, alumina was chosen for the waveguide in our SPARROW biosensor structure. However, alumina is polycrystalline structure and has amorphous surface. For the fundamental study of organic film absorption on $\mathrm{Al}_{2} \mathrm{O}_{3}$, the surface condition is required to be as simple as possible. In addition, when taking the AFM image, the surface roughness of the alumina waveguide (on the order of $1 \mathrm{~nm}$ ) is comparable to the surface roughness of the SAMs $(\sim 2 \mathrm{~nm})$. This makes it difficult to distinguish the biolayer surface roughness from the waveguide surface roughness. Thus, we also explored the use of sapphire. Sapphire can be prepared with a much smoother surface since it is aluminum oxide in crystalline form (the crystal structure is hexagonal), however, it has not been used in the actual SPARROW biosensor for economical purpose (i.e., A very expensive device - Mental Organic Chemical Vapor Deposition (MOCVD) is required to grow sapphire, which greatly increase the costs of biosensor). Generally, the sapphire wafers used here were c plates (i.e., wafer surfaces are (0001) planes, approximately). We obtained the optical constants of the alumina by the

ellipsometry technique and determined the surface topographies of both alumina and sapphire by using AFM after sample preparation. 


\section{Bare sapphire $\left(\mathrm{Al}_{2} \mathrm{O}_{3}\right)$}

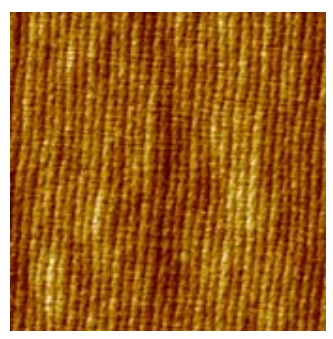

Size of images: $3 \mu \mathrm{m} \times 3 \mu \mathrm{m}$.

\section{Bare alumina $\left(\mathrm{Al}_{2} \mathrm{O}_{3}\right)$}

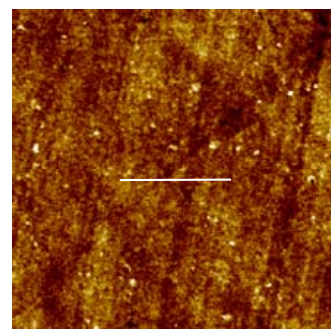

Size of images: $3 \mu \mathrm{m} \times 3 \mu \mathrm{m}$.
RMS (Root-Mean-Square) roughness $\sim 0.074 \mathrm{~nm}$

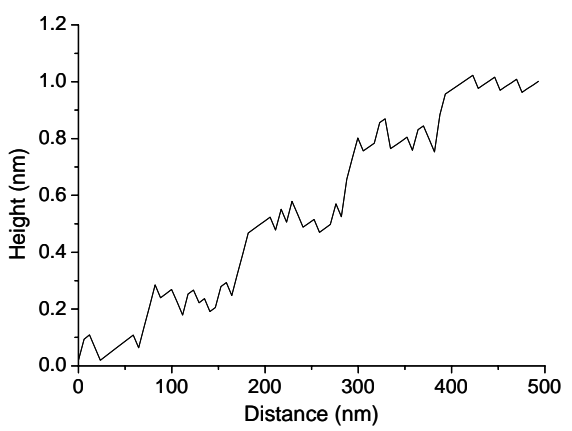

RMS roughness $\sim 0.63 \mathrm{~nm}$

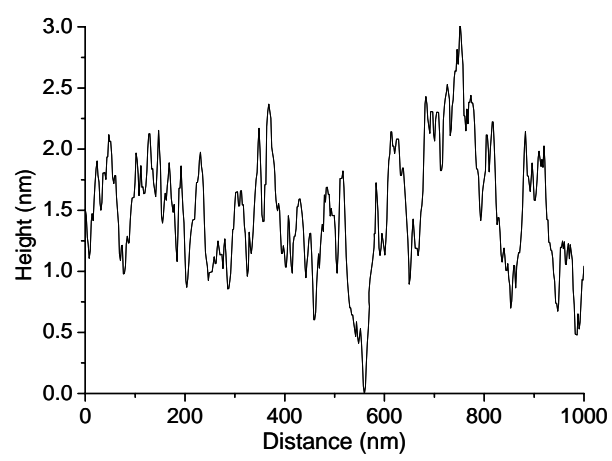

Fig. 4.11. AFM images and surface roughness from sapphire and bare alumina surfaces after cleaning.

Figure 4.11 shows the AFM images of the alumina and c-plane sapphire surfaces after sample preparation. In this preparation, all wafers of alumina and sapphire were cut into small square pieces by a diamond scribe. To get rid of the contaminants on the wafer surface, they were cleaned by ultrasonication in toluene, acetone, hexane, and isopropanol for 60 minutes. Sapphire and alumina were treated differently afterwards, because sapphire is a homogeneous single crystal while alumina is a thin film deposited on a glass substrate. The sapphire substrates were etched in a $1 \mathrm{H}_{2} \mathrm{SO}_{4}+1 \mathrm{H}_{2} \mathrm{PO}_{4}+5 \mathrm{H}_{2} \mathrm{O}$ solution for 30 minutes at $70^{\circ} \mathrm{C}$, rinsed in de-ionized (DI) water, dried in argon gas, and annealed at a high temperature $\left(\sim 1050^{\circ} \mathrm{C}\right)$ in air. The annealing process reduces the density of surface defects, such as steps, kinks, and atomic vacancies and makes the 
surface much smoother. Alumina substrates, however, were just incubated in a furnace at $250^{\circ} \mathrm{C}$ in air for 30 minutes and used for growing SAM without further treatment. The AFM images and corresponding cross-sectional plots of sapphire and alumina surfaces in Fig. 4.10 were taken right before these substrates were used for growing SAMs. Rootmean-square (RMS) roughnesses of both surfaces were less than 1 nanometer. This RMS roughness directly links to the device behavior discussed in Chapter 5 .

Ellipsometry offers another way to measure the surface roughness. Also, the optical constants of alumina could be determined. In our measurements, for both alumina and sapphire samples, the surface roughness from ellipsometry was around 1-2 nm (a little different from AFM results). We believe this is caused by limitations introduced by the number of fitting parameters (See Fig. 4.3, less fitting parameter will be more accurate). At a wavelength of $633 \mathrm{~nm}$, the refractive indices of the alumina sample greatly depended on depositing condition. For different conditions, it varies from 1.60 to 1.65, according to Ellipsometry measurements. Absorption can also be obtained by index imaginary part $K$ by Eq. $4-2$.

$$
\alpha_{\text {absorption }}=\frac{4 K}{\lambda_{\text {vac }}}
$$

In general case, the absorption in the alumina sample was small and could be neglected.

\section{4-3-2 Self-assembled monolayers (SAMs)}

SAMs are used for electrochemical, optical, and other detection systems. In our biosensor device, they are used as the connecter between the biolayer and transducer. SAMs are molecular assemblies that are formed spontaneously by the immersion of a solid substrate into a solution of an active surfactant in an organic solvent. Many kinds of SAMs have been studied by number of authors (Ruhe, et al., 1993; Ulman, 1996; Messerschmidt, et al., 2001; Biddle, 2001; Kosuri, et al., 2004) for different substrate binding. From literatures (Allara, et al., 1985; Tao, et al., 1993; Taylor, et al., 2003; Devaprakasam, et al., 2004), stearic acid, a special kind of SAMs, can be used to form a very good layer on sapphire and alumina surface. This has been proven in the WVU Dept. of Chemistry under the supervision of Dr. Minsoo Lim (Lim, et al., 2006). We could easily achieve almost full surface coverage and a very smooth surface for this kind 
of SAMs. As shown on Fig. 4.12, the growth of stearic acid doesn't increase the Root Mean Square (RMS) roughness. It means the surface coverage is close to either $0 \%$ or $100 \%$. The difference between no coverage and full coverage can be easily distinguished by FTIR and contact angle measurements which will be mentioned in later of this section.

However, stearic acid will be quickly desorbed in a water environment, which is required for SPARROW device operation. Such property has been found in our contact angle experiments. Since bare sapphire is hydrophilic and aminosilane is hydrophobic, the contact angle will show significant different from no coverage (approximate $10^{\circ}$ ) to full coverage (approximate $90^{\circ}$ ). In the series of tests of stearic acid stability in water environment, the contact angle, which is proportional to surface coverage, decreases as a function of water incubation time (Fig. 4.13). Another choice for an optimum SAM was then needed.

SAMs of stearic acid grown on sapphire

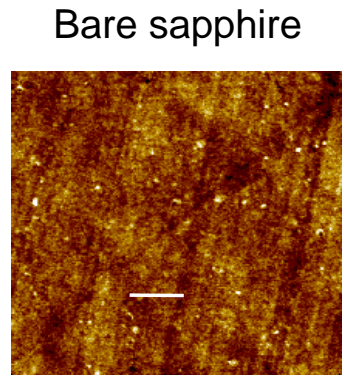

$3 \mu \mathrm{m} \times 3 \mu \mathrm{m}$

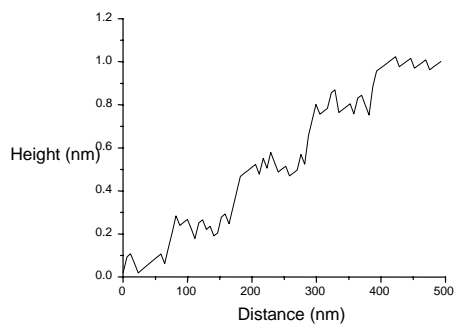

RMS roughness: $0.074 \mathrm{~nm}$
165.5 hour growth

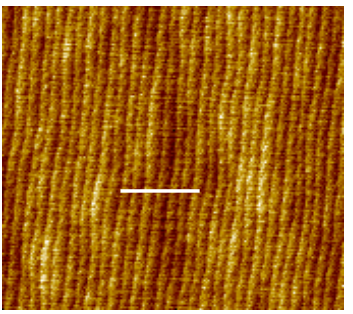

$3 \mu \mathrm{m} \times 3 \mu \mathrm{m}$

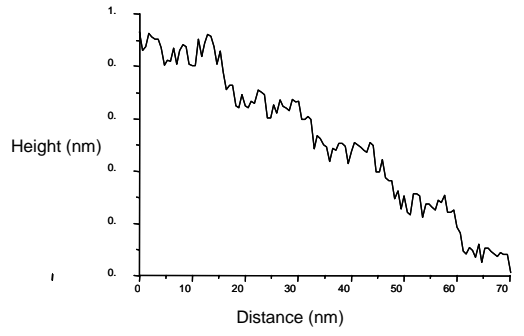

RMS roughness: $0.069 \mathrm{~nm}$

Fig. 4.12. AFM images of stearic acid grown on sapphire. The units for RMS roughness are in $\mathrm{nm}$. 


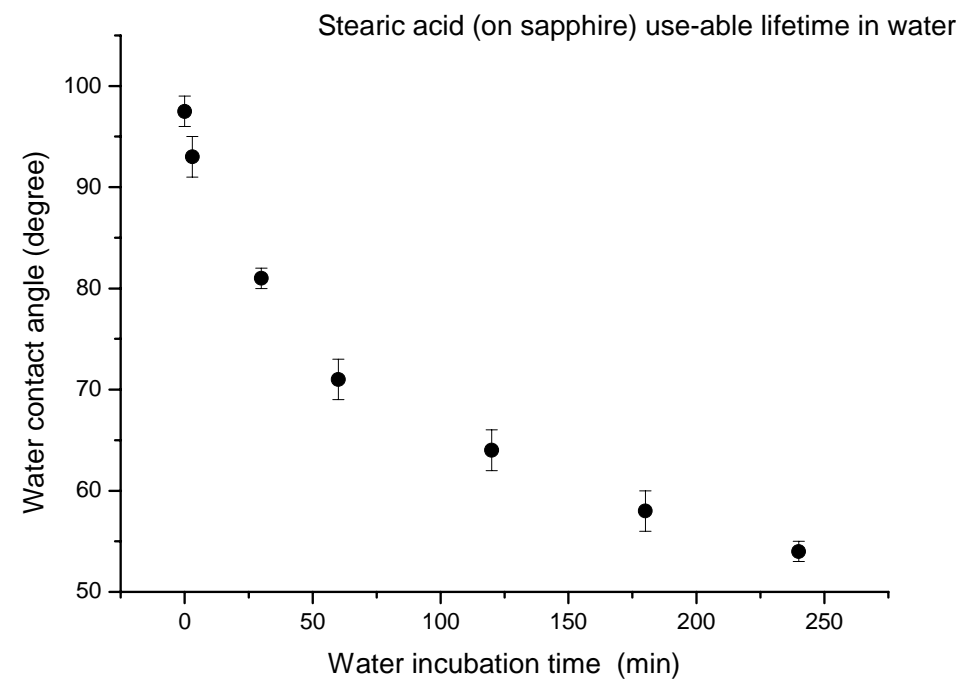

Fig.4.13. Stearic acid lifetime in water

After testing several other kinds of SAMs, such as phosphoric acid and aminosilane, we found that aminosilane also exhibits smooth surfaces. More importantly, aminosilane was highly stable in a water environment. From Dow Corning Co. silane solution page, aminosilane has the following properties

* Adhesion promoter, coupling agent, and resin additive

* Improves chemical bonding of resins to inorganic fillers and reinforcing materials

* Used for epoxies, phenolics, melamines, nylons, PVC, acrylics, polyolefins, polyurethanes, and nitrile rubbers

* Surface pretreatment of fillers and reinforces

In our experiments, the aminosilane structure, $\mathrm{NH}_{2}\left(\mathrm{CH}_{2}\right)_{11} \mathrm{Si}\left(\mathrm{OCH}_{2} \mathrm{CH}_{3}\right)_{3}$, is bound to the sapphire and alumina surface by the Si-O bond. Another terminal is $\mathrm{NH}_{2}$ which was used to connect succinic anhydride which directly links to streptavidin. The following graphs show the AFM images of aminosilane grown on sapphire (Fig. 4.14) and grown on alumina (Fig. 4.15). Also, the stability in a water environment was determined using contact-angle measurements. The results are shown in Figs. 4.16 and 4.17. 

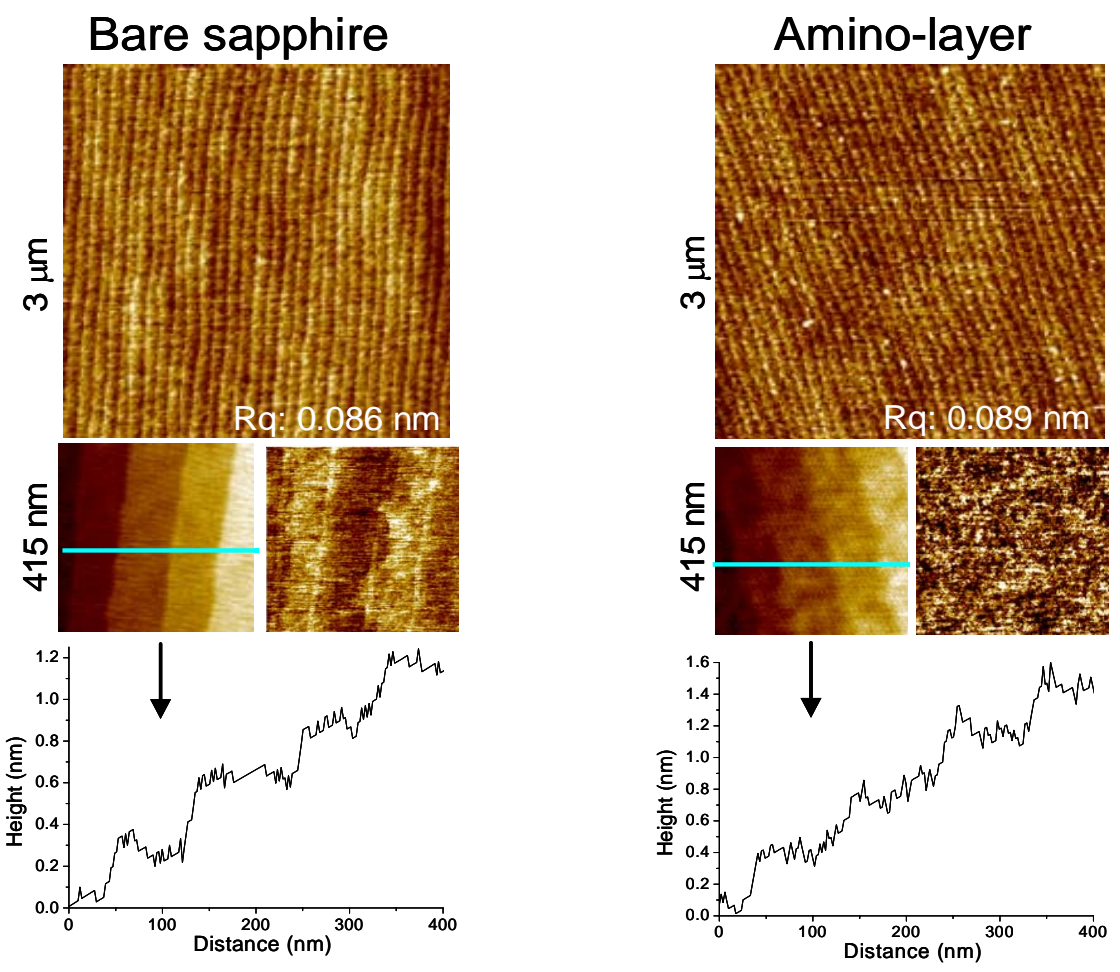

Fig. 4.14. AFM images of aminosilane on sapphire.
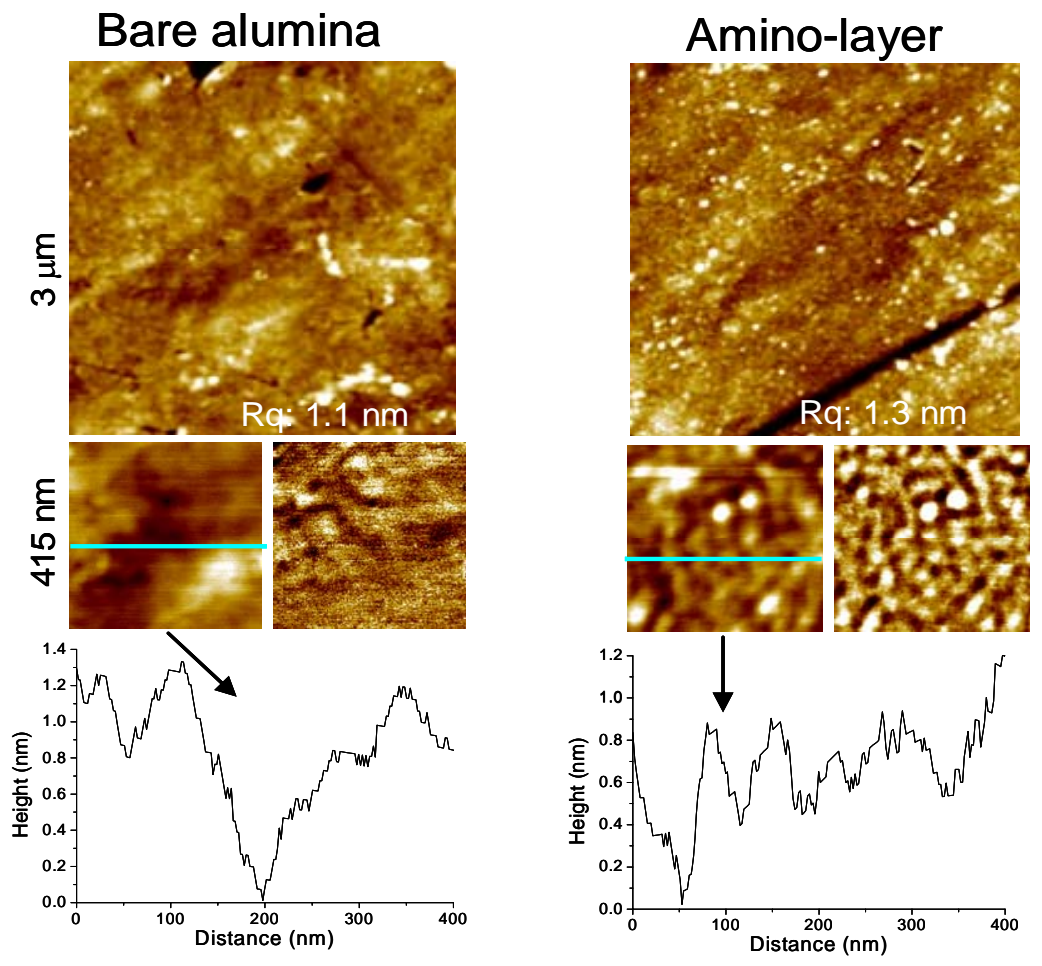

Fig. 4.15. AFM images of aminosilane on alumina. 


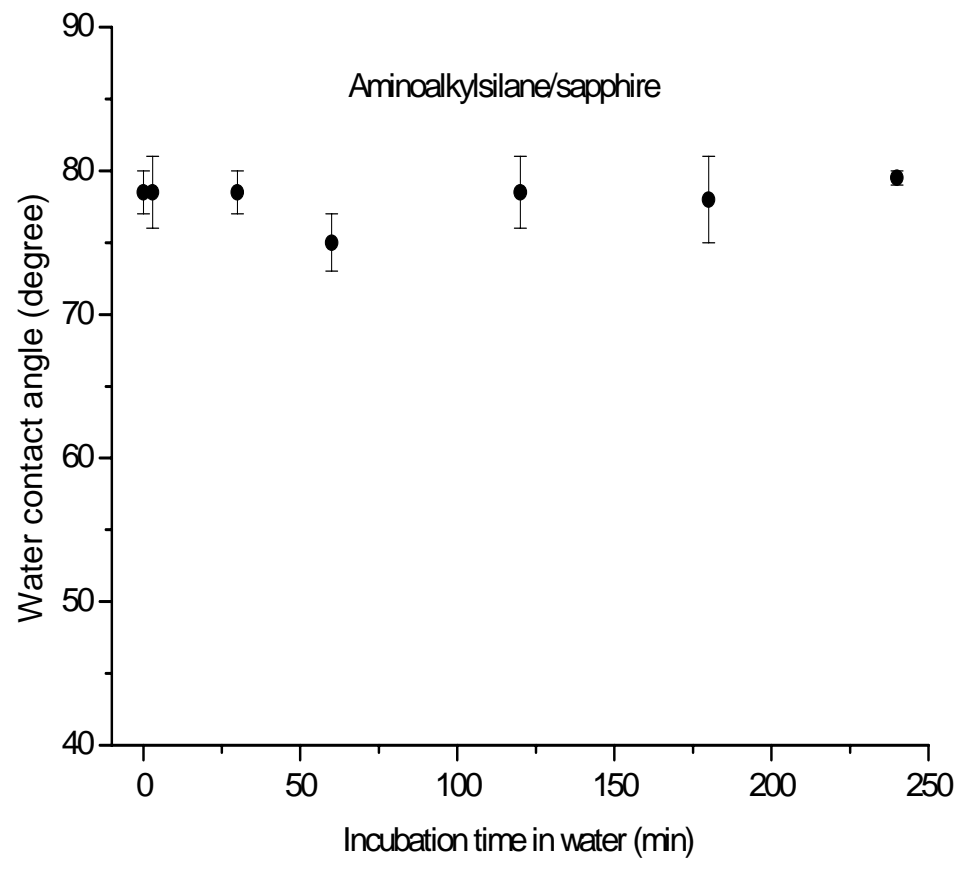

Fig. 4.16. Stability of aminosilane/sapphire in water.

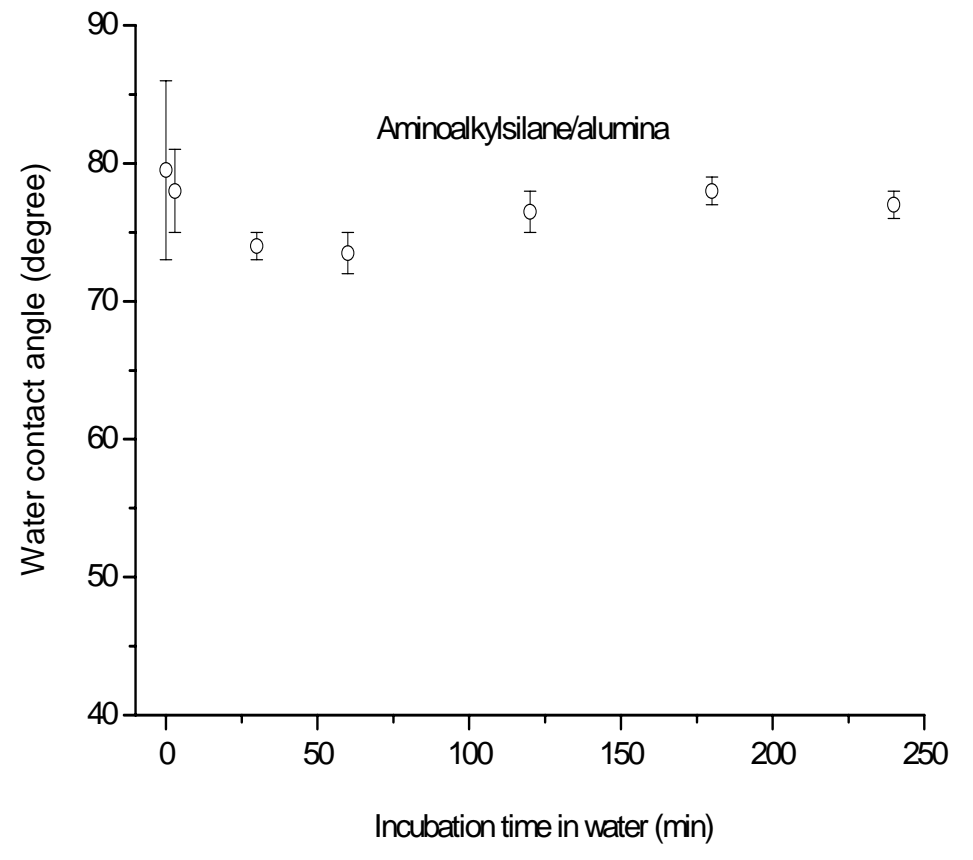

Fig. 4.17. Stability of aminosilane/alumina in water. 
According to contact-angle graphs and the AFM images, aminosilane shows very good stability, as depicted by the non-changing contact angle. AFM images after varying water incubation times also show the stability of aminosilane in a water environment (Fig. 4.18).
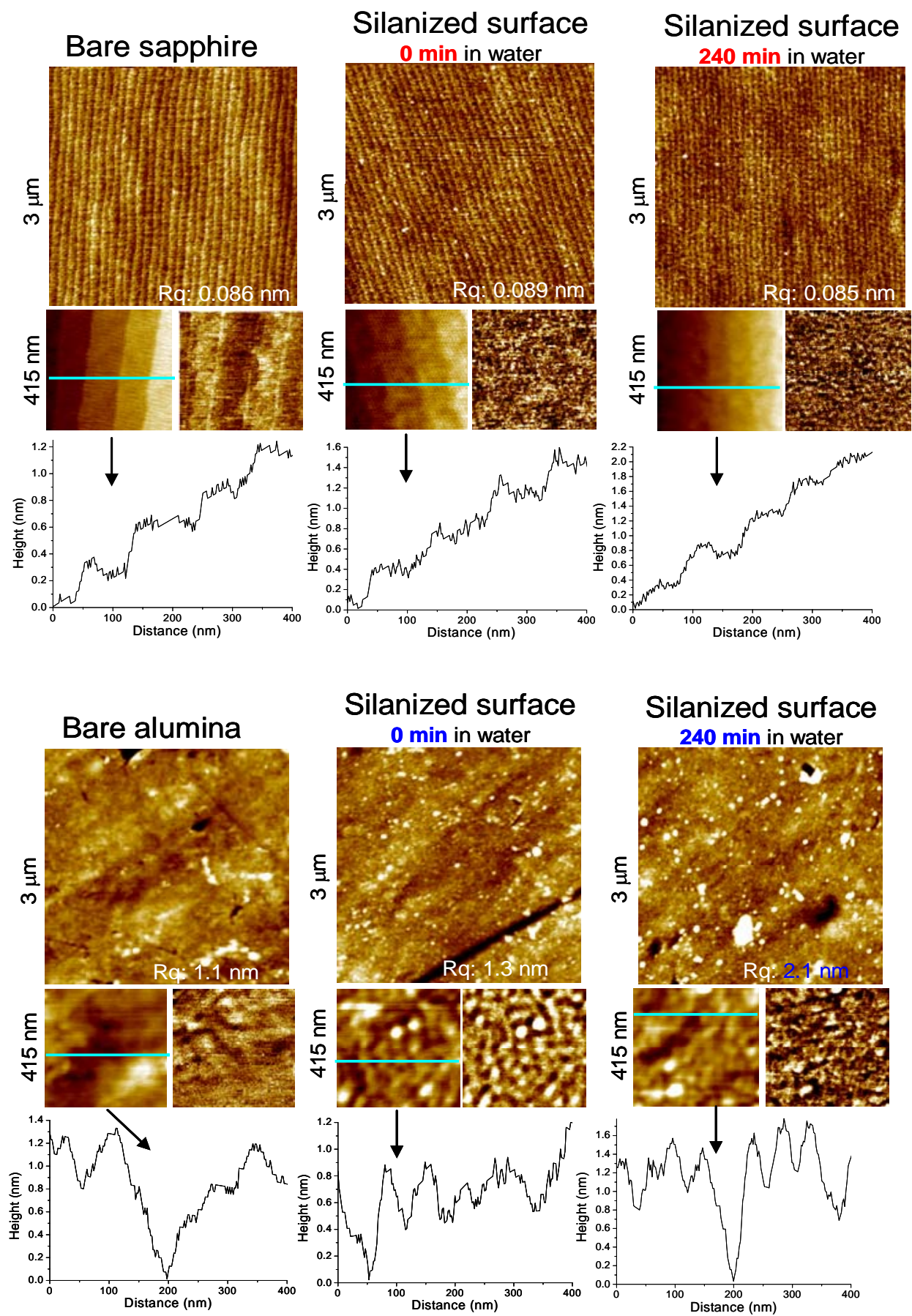

Fig. 4.18. AFM images of an aminosilane sample after water incubation. 
Without a doubt, AFM offers a very important technique for detection of surface information. However, it has limitations in its ability to identify specific biomaterials, such as distinguishing aminosilane from contaminants. If their surface tensions are different, contact angle measurements will quickly tell the results. However, if their surface tensions remain unknown or are close, other methods have to be considered. In our lab, FTIR measurements were used to detect and monitor the infrared absorptions due to $\mathrm{CH}_{2}$. Because aminosilane includes a long $\mathrm{CH}_{2}$ chain, once characteristic absorption bands related to $\mathrm{CH}_{2}$ stretching or vibrational modes are observed, then it could be determined that there was aminosilane on the surface, instead of some other material. In the FTIR spectrum, the peaks associated with $\mathrm{CH}_{2}$ absorptions occur at the wave numbers $2850 \mathrm{~cm}^{-1}$ and $2917 \mathrm{~cm}^{-1}$ (Taylor, et al., 2002). These peaks correspond to the $\mathrm{CH}_{2}$ bands of symmetric stretch and asymmetric stretch, respectively. Fig. 4.19. illustrates how this stretch occurs (Silverstein, et al., 1981).
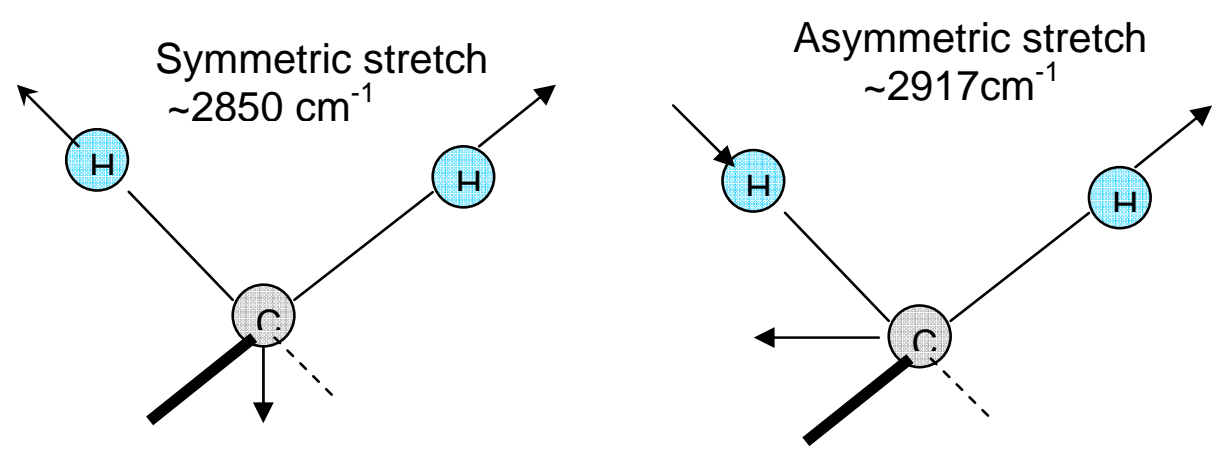

Fig. 4.19. Schematic of two types of stretch of $\mathrm{CH}_{2}$ bands

At room temperature, a spectrum measured from a silane coated sapphire is shown in Fig. 4.20. Both absorbance and transmittance spectrums are shown. Easily, we can find the peak occurs at $2850 \mathrm{~cm}^{-1}$ and $2917 \mathrm{~cm}^{-1}$, which are exactly the same as reference. It proves that silane is successfully grown on sapphire. 

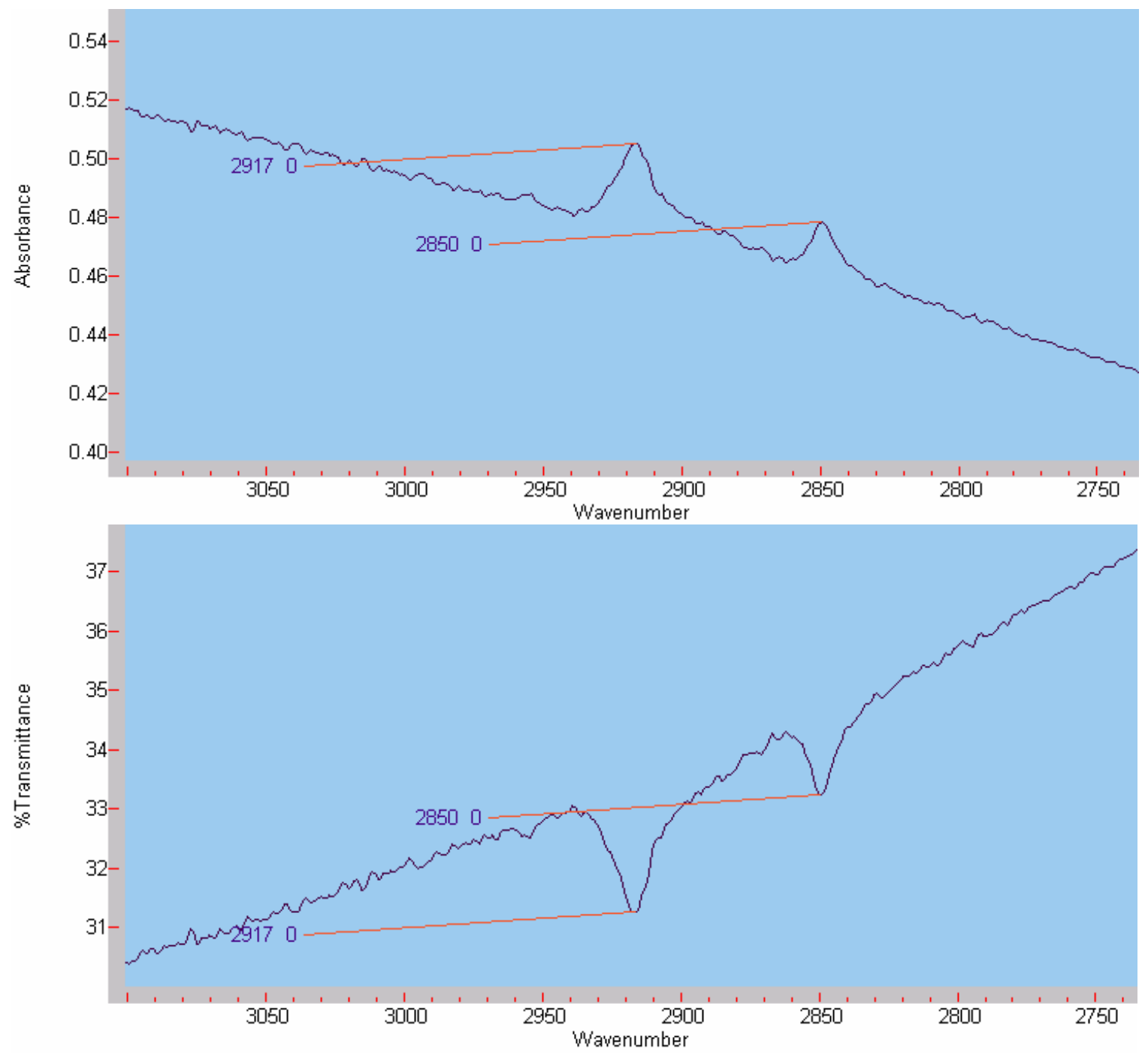

Fig. 4.20. $\mathrm{CH}_{2}$ symmetric and asymmetric stretch bands in FTIR spectra.

\section{4-3-3 Streptavidin}

Streptavidin is a tetrameric protein purified from Streptomyces avidinii. The longitudinal thickness of this material is around $10 \mathrm{~nm}$. It can bind to biotin very tightly, forming what is called a streptavidin/biotin complex (Avidin-Biotin Chemistry, 1994). This complex has one of the strongest biochemical interactions known and is also extremely stable over a wide range of temperature and $\mathrm{pH}$. Due to its distinctive properties, the streptavidin/complex is taken advantage of in scientific laboratories (Schiestel, et al., 2004). In the SPARROW biosensor, streptavidin could be used to detect micro-molecules, such as anthrax spores. Experimentally, we grew streptavidin on the 
amino surface (Fig. 4.21) by using both sapphire and alumina substrates. Contact-angle measurements and AFM images show this material also has good stability in a water environment. These graphs are shown in Fig. 4-22, 4-23, 4-24 and 4-25, respectively.
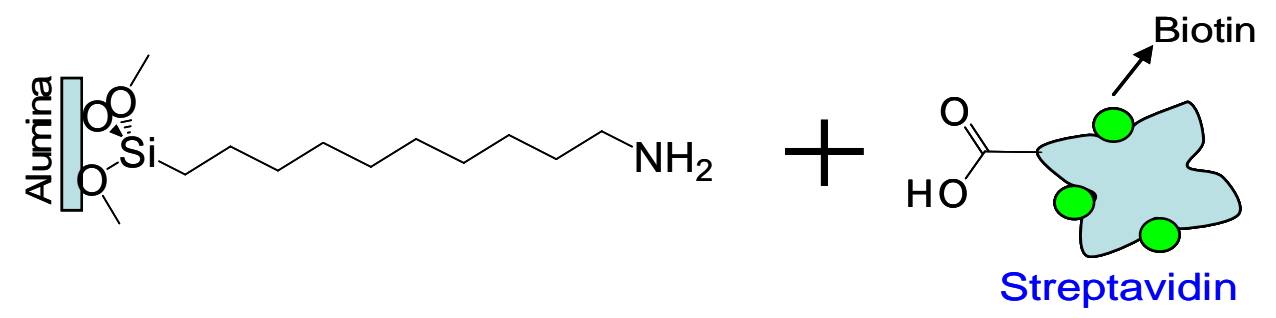

Fig. 4.21. Streptavidin link to amino surface.

Immobilization of streptavidin on amino-sapphire

Amino-sapphire Streptavidin on amino-sapphire
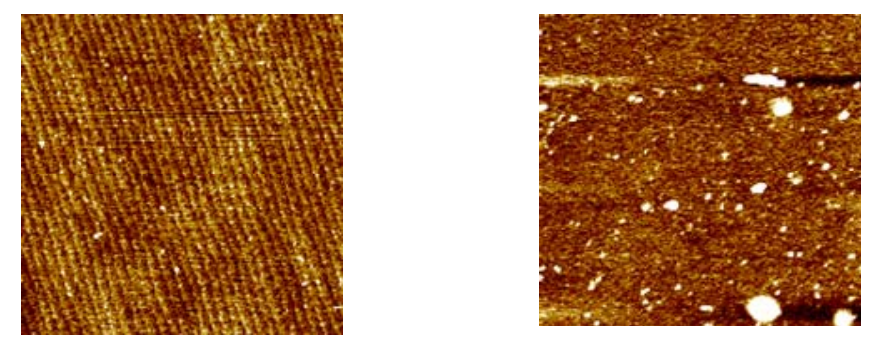

$3 \mu \mathrm{m} \times 3 \mu \mathrm{m}$

$3 \mu \mathrm{m} \times 3 \mu \mathrm{m}$

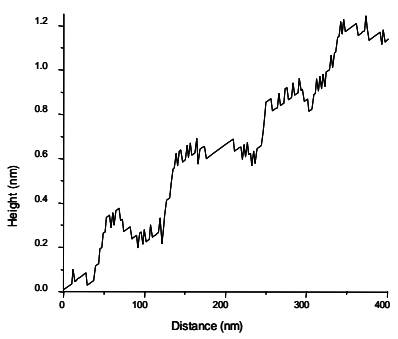

RMS roughness: $0.089 \mathrm{~nm}$

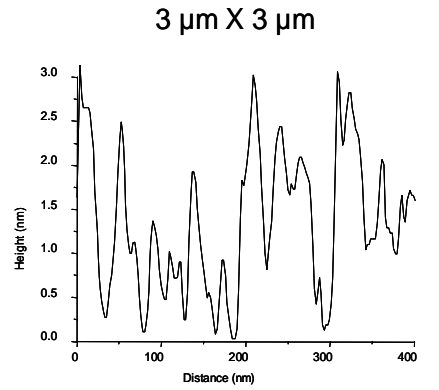

RMS roughness: $1.54 \mathrm{~nm}$

Fig. 4.22. AFM images of streptavidin on amino-sapphire. 


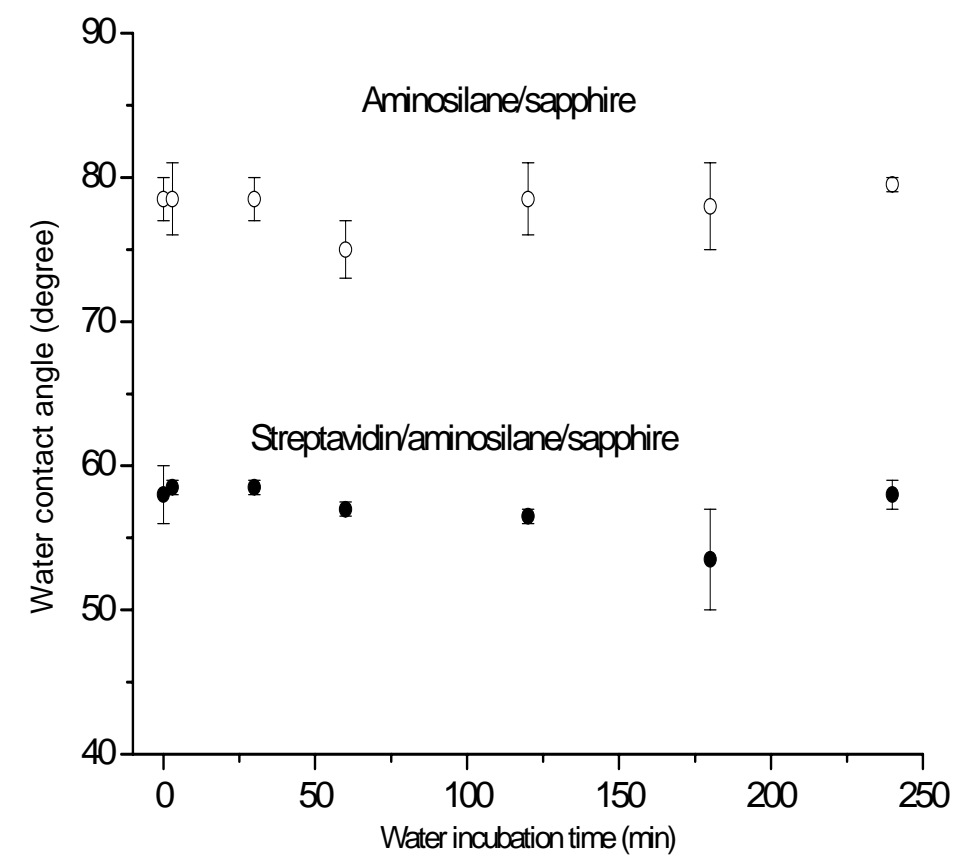

Fig. 4.23. Stability of streptavidin/aminosilane/sapphire in water environment and contact angle comparison Immobilization of streptavidin on amino-alumina

Amino-alumina

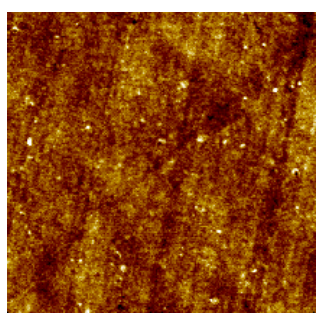

$3 \mu \mathrm{m} \times 3 \mu \mathrm{m}$

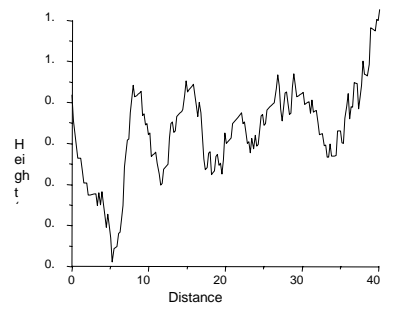

RMS roughness: $1.3 \mathrm{~nm}$
Streptavidin on amino-sapphire

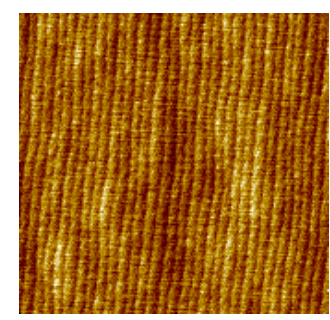

$3 \mu \mathrm{m} \times 3 \mu \mathrm{m}$

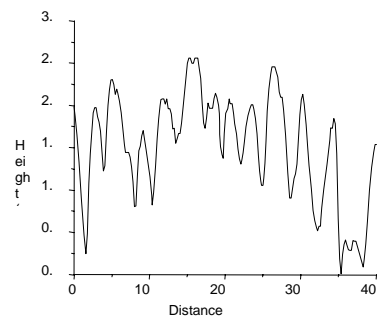

RMS roughness: $1.1 \mathrm{~nm}$

Fig. 4.24. AFM images of aminosilane on alumina. 


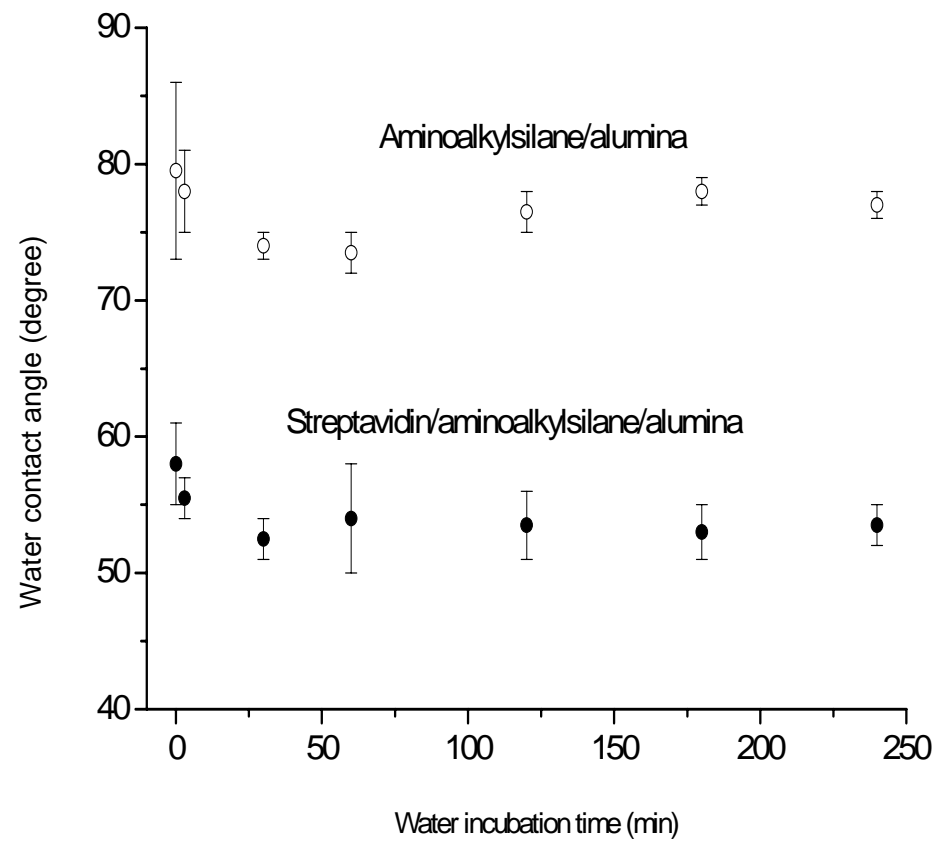

Fig. 4.24. Stability of streptavidin/aminosilane/alumina in water environment and contact angle comparison

\section{4-3-4 Biotin conjugated microsphere beads}

The purpose of the SPARROW biosensor is to detect the specific binding events. To perform a functional test, biotin conjugated microsphere latex beads were used in an experiment to simulate the anthrax spores. When the medium containing the micro-beads flows through the PDMS channel, some of the beads will chemically bind to the biolayer. This leads to a change in the coupling coefficient and coupling length. The bead coverage will depend on the flow rate, micro-bead concentration in the solution medium, and the flow time. The distribution of the micro-beads on a PDMS channel was monitored using a fluorescent microscope (data provided by Dr. Minsoo Lim). Representative fluorescent images are shown in Figs. 4.25. and 4.26 for two different micro-bead concentrations and two different flow rates. The size of these beads is detected by AFM (Fig. 4.27). Generally, the beads size obtained from AFM images is much less than their nominal size $(1 \mu \mathrm{m})$ because the beads will shrink after leaving water environment. In this image, the average beads' width is approximate $1.3 \mu \mathrm{m}$ and 
the height is only $42 \mathrm{~nm}$.

Concentration: $7.2 \times 10^{7}$ particles $/ \mathrm{ml}$
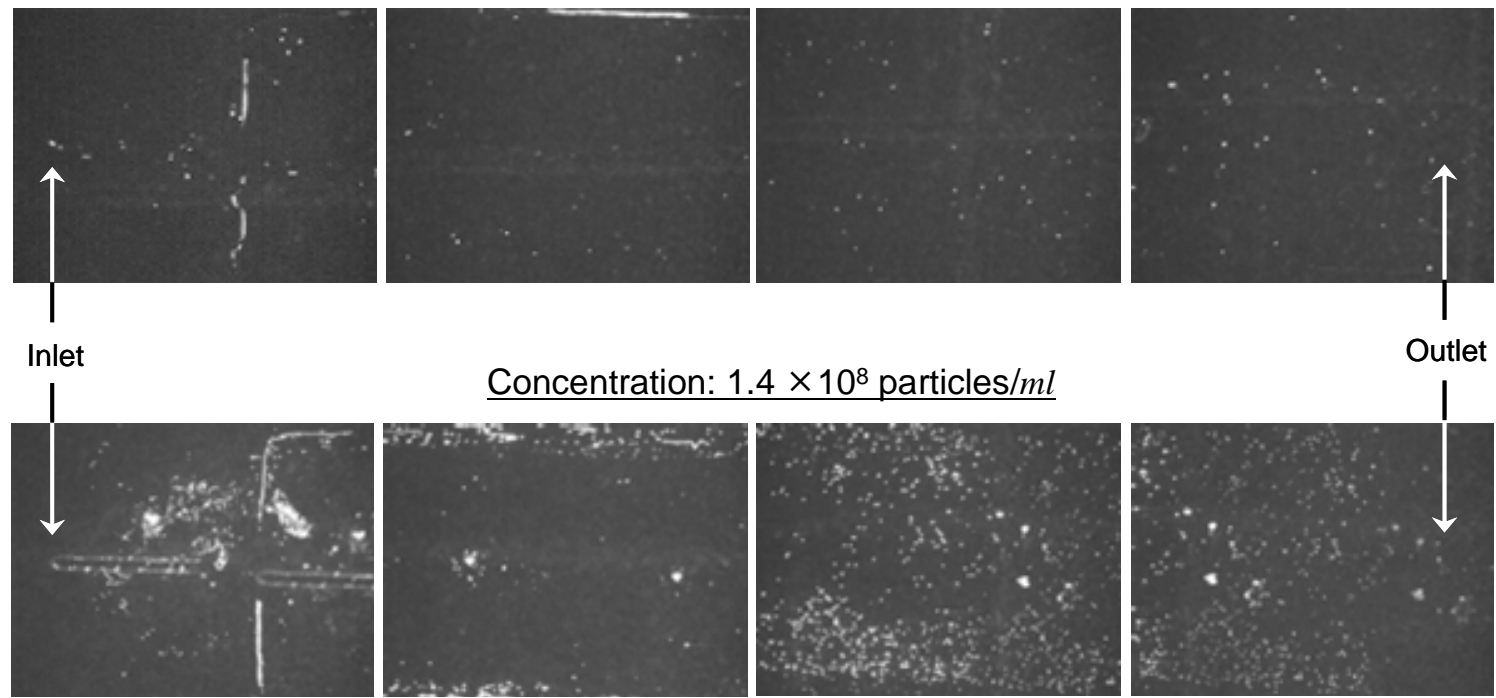

Concentration: $1.4 \times 10^{8}$ particles $/ \mathrm{ml}$

Outlet
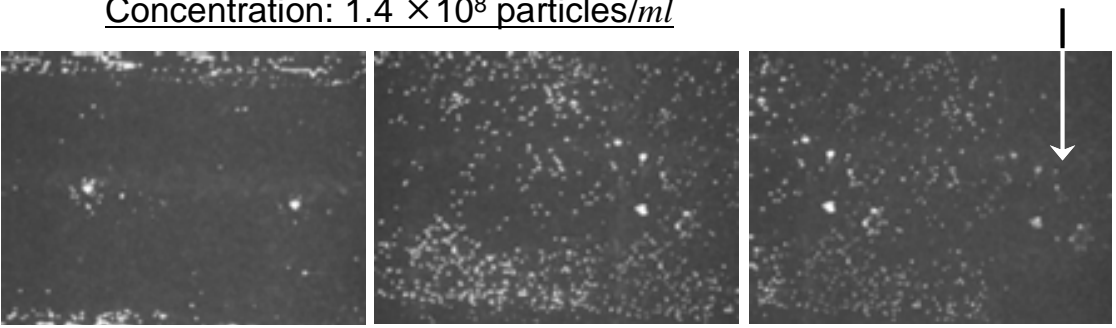

Fig 4.25. Fluorescent microscope images of functionalized beads on biolayer prepared using a flow rate of $0.5 \mu \mathrm{l} / \mathrm{min}$.

Concentration: $7.2 \times 10^{7}$ particles $/ \mathrm{ml}$
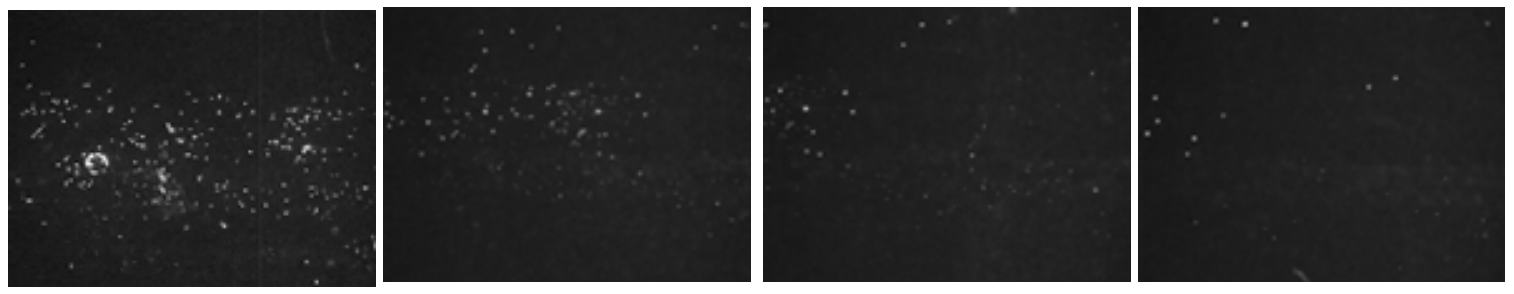

Concentration: $1.4 \times 10^{8}$ particles $/ \mathrm{ml}$
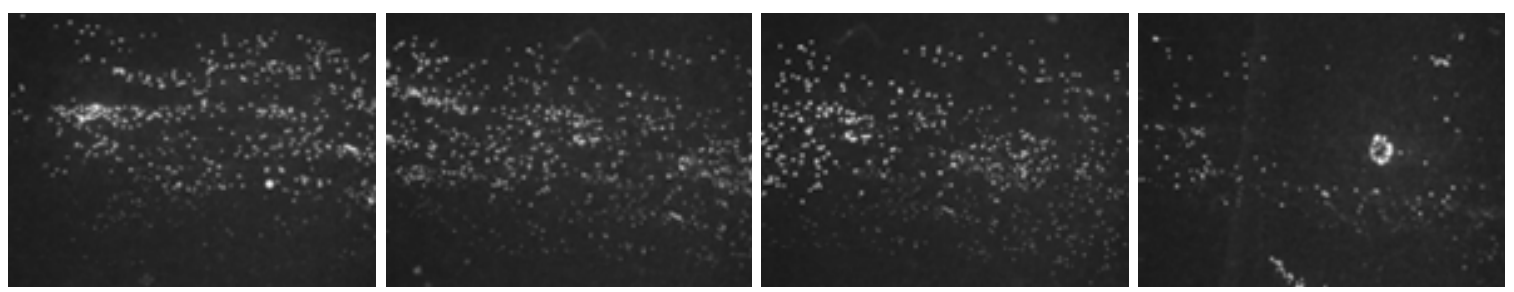

Fig. 4.26. Fluorescent microscope images of functionalized beads on biolayer prepared using a flow rate of $1.5 \mu \mathrm{l} / \mathrm{min}$. 


\section{Microbeads (nominal diameter: $1 \mu \mathrm{m}$ ) bound to streptavidin layer}

- Exposure time of microbeads solution to sapphire surface: $10 \mathrm{~min}$.

- Bead concentration: $7.2 \times 10^{7}$ particles $/ \mathrm{mL}$
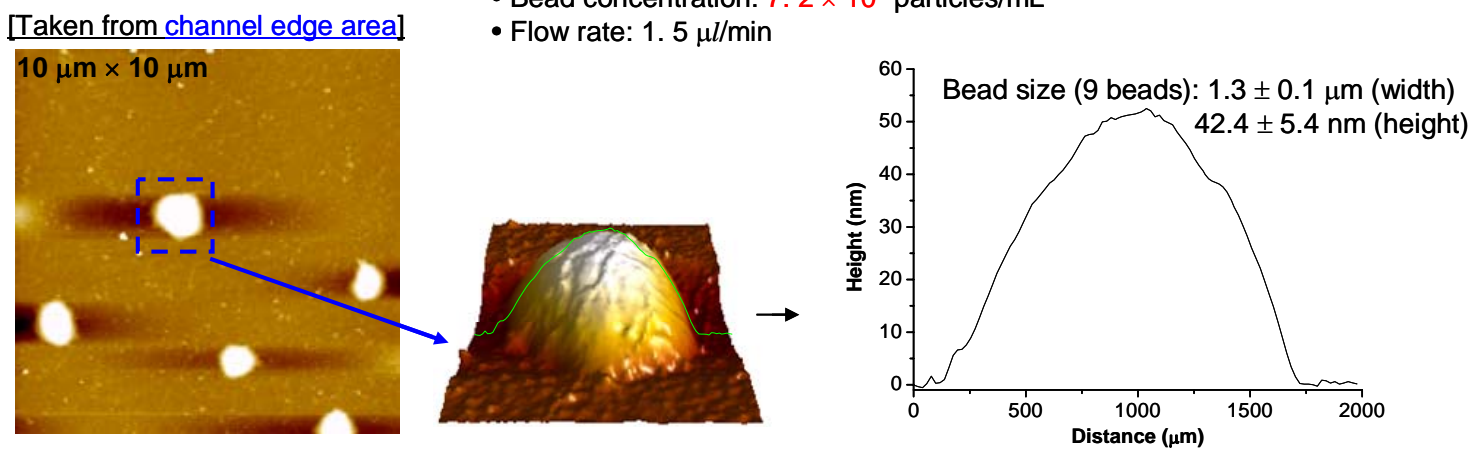

Fig. 4.27. Size of microbeads by AFM detection

With these data information, further theoretical calculation can be performed. In the next chapter, an analytical model is developed and numerical results are obtained using the information described in the present chapter. 


\section{Chapter 5}

\section{Non-ideal SPARROW biosensor modeling}

In Chapter 3, the mathematical modeling for an ideal SPARROW biosensor was presented. However, due to fabrication limitations, variations in waveguide sidewall roughness, biolayer surface roughness, and biolayer partial coverage, there can be a wide variation in biosensor behavior. In addition, to be able to interpret the device performance correctly, the influence of random surface loading of molecular binding events has to be considered. In this chapter, these effects on biosensor behavior are discussed and a general analytical model for non-ideal biosensor behavior is presented.

\section{5-1 Scattering loss in waveguide}

Generally, when light travels in a waveguide, some of its energy will leak into the cladding and substrate layers. Therefore, the total power will not be conserved when we measure it at the input and output ends. This phenomenon is referred to as power attenuation. This attenuation can be caused by absorption or scattering. In the SPARROW biosensor structure, the light source is a He-Ne laser and the wavelength is $633 \mathrm{~nm}$. At this wavelength, an alumina waveguide shows very little absorption loss (absorption coefficient $\alpha_{\text {absorption }}=4 K / \lambda$ ). In this dissertation work, this absorption loss was ignored because it can be considered as negligible compared to scattering/radiation losses which dominate the total power loss. Thus, in this section, we will focus on how the scattering/radiation loss affects biosensor behavior.

The scattering loss is defined as the part of the transmission loss that results from scattering within a transmission medium or from roughness of a reflecting surface. From this definition, there are two factors that lead to scattering: one is an inhomogeneous refractive index of the waveguide and the other is surface roughness. These two issues will be separately addressed.

In real devices, the waveguide layers may be slightly inhomogeneous. When the light propagates in such a waveguide (see Fig. 5.1), part of energy will transform to radiation modes or other modes due to scattering caused by refractive index 
inhomogeneities. Generally, this scattering is called Rayleigh scattering. The mathematical model of this kind of scattering can be found in many literatures (Imai, et al., 1977; Yamakita, et al., 1980; Wang, et al., 1998; Garcia-Martin, et al., 2005; etc.). In a single waveguide, the approximate Rayleigh scattering is given by the expression (Palais, 2002)

$$
S_{L}=1.7 *(0.85 / \lambda)^{4}
$$

In a single-mode waveguide, the average Rayleigh scattering loss is (Liang, 1992)

$$
\bar{\partial}_{s}=\iint_{A} \partial_{s}(x, y) E^{2}(x, y) d x d y
$$

where $\partial_{s}(x, y)$ is the Rayleigh scattering loss at the point $(\mathrm{x}, \mathrm{y})$ in the waveguide cross section. This can be written as

$$
\begin{array}{rlrl}
\partial_{s}(x, y) & =C_{1}\left[n^{2}(x, y)-1\right] / \lambda^{4} \quad & \text { core } \\
& =C_{2}\left[n_{2}^{2}-1\right] / \lambda^{4} \quad \text { cladding }
\end{array}
$$

where $C_{1}$ and $C_{2}$ are the Rayleigh scattering coefficients of the core and the cladding layers, respectively.

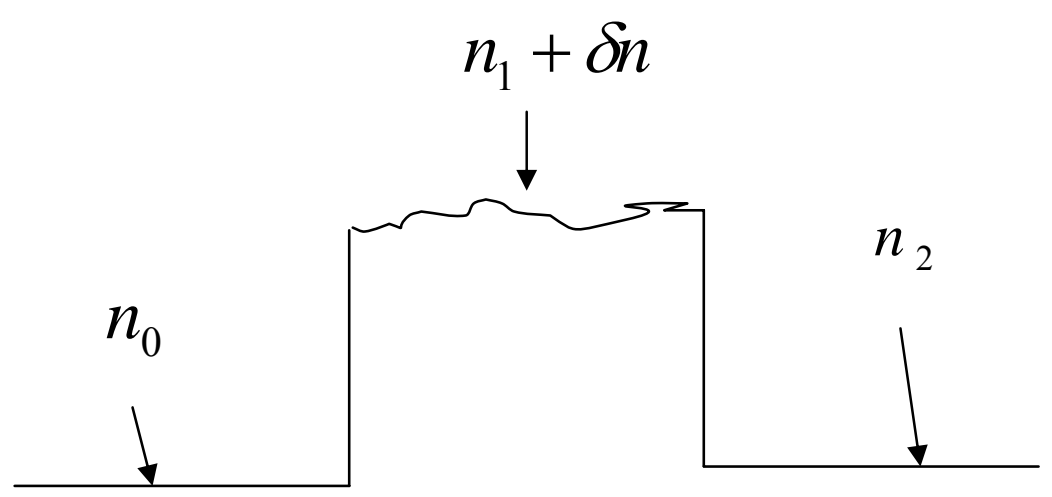

Fig. 5.1. Schematic depiction of refractive index fluctuations in a waveguide. 


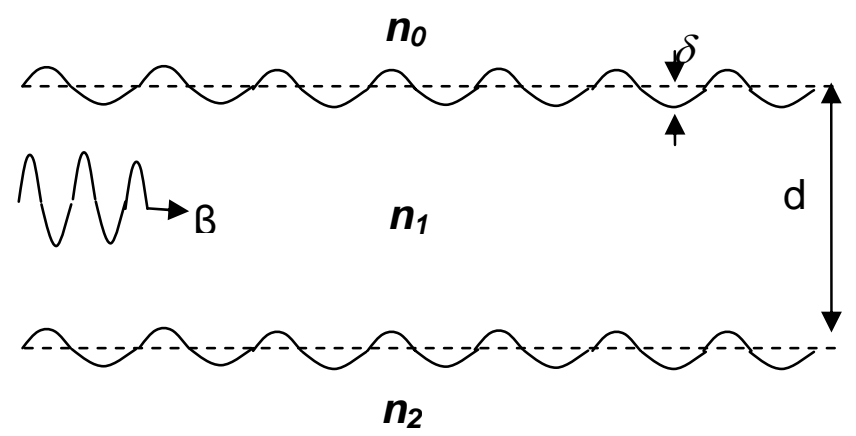

Fig. 5.2. Waveguide with surface roughness.

Scattering due to surface imperfections (see Fig. 5.2) can also occur in real waveguide layers. Techniques for analyzing this scattering loss have been developed by several authors using different methods based on perturbation analysis of the normal modal field (Trillo, et al., 1986; Lacey, 1990; Ishimaru, 1991; Ladouceur, 1994,1995,1996,1997; Jin, et al., 1996; Chimenti, 1998). This perturbation theory that serves as the basis of calculation is expected to be valid when $\delta<<\mathrm{h}$ ( $\delta$ is the surface roughness varies above and below this boundary, $h$ is the average thickness of waveguide), which is the case for most good quality planar waveguides. To deal with this problem, relationship between any two random points along a waveguide surface has been considered in all methods. It gives two equations as

$$
<\delta>=0
$$

and

$$
<f(z) f(z+u)>=C(u)
$$

Eq. 5-5 is used to describe a uniform boundary. Surface roughness varies above and below this boundary such that the average is zero. Eq. 5-6 is known as the autocorrelation function. It describes the relationship between two different points along the surface separated by a distance $u$. Furthermore, if the surface exhibits an exponential correlation (Ogilvy, 1989), this equation can be written as

$$
C(u)=<f(z) f(z+u)>=\sigma^{2} \operatorname{Exp}\left(-|u| / l_{c}\right)
$$

In the Gaussian-correlated case, it can be expressed as 


$$
C(u)=<f(z) f(z+u)>=\sigma^{2} \operatorname{Exp}\left(-u^{2} / l_{c}^{2}\right)
$$

where $l_{c}$ is the correlation length in both equations. Mathematically, it is defined as the distance over which $C(u)$ falls to $e^{-1}$. When $l_{c}$ goes to 0 , the spectral power density will be a constant for all frequencies, i.e., independent of frequency, so can be referred to as "white noise". When $l_{c}$ goes to infinity, the roughness is smoothed out (Ladouceur, 1997). The parameter $\sigma^{2}$ is the standard deviation of roughness which is equal to $<\delta^{2}>$. Also, we call $\sigma^{2}$ as the root-mean-square (RMS) roughness.

Sample waveguide surface graphs (from Ladouceur, 1994) are shown in Fig. 5.3. The four graphs represent four different locations in the same waveguide. Here, a total of 512 points were measured, implying the sample interval is $10 \mathrm{~nm}$. The RMS roughness of these four regions is $40 \mathrm{~nm} \pm 5 \mathrm{~nm}$. The correlation length is $230 \mathrm{~nm} \pm 50 \mathrm{~nm}$.
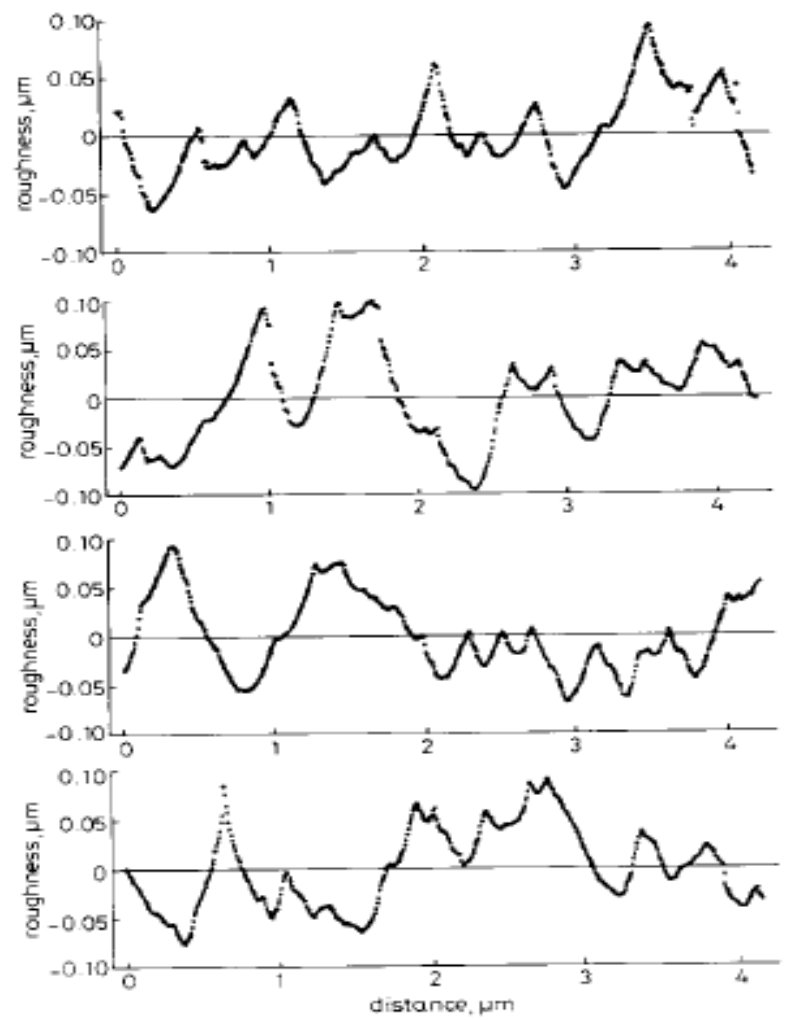

Fig. 5.3. Sample waveguide surface graphs in four different regions (from Ladouceur, 1994). 
To connect a surface roughness and a surface correlation to a scattering loss, a Fourier transform is performed and another important quantity, the power spectrum $\mathbf{S}(\gamma)$, is generated. It is the average intensity of the Fourier component of spatial frequency $\gamma$, and is given in Eq. 5-9.

$$
\begin{gathered}
C(u)=\int_{-\infty}^{\infty} e^{i \eta \mu} S(\gamma) d \gamma \\
S(\gamma)=\frac{1}{2 \pi} \int_{-\infty}^{\infty} e^{-i j u} C(u) d u
\end{gathered}
$$

Equation 5-7 works very well when the sample interval is smaller than one tenth of the surface correlation length. However, when the interval increases, this approximation will no longer be valid. In this case, Eq. 5-8 is a better choice. If the sample interval is too large, the surface correlation will be neither exponential nor Gaussian. There will be a huge error if we still use these correlation functions. For this latter case, some other methods have to be considered (Chen, et al., 1988). When the surface is exponential, combining Eqs. 5-7 and 5-9, we get the power spectrum in the form of a Lorentzian.

$$
S(\gamma)=\frac{\delta^{2}}{\pi} \frac{l_{c}}{1+l_{c}^{2} \gamma^{2}}
$$

As $l_{c} \rightarrow 0$, we will have a constant power spectrum. When the surface is Gaussian, combining Eqs. 5-8 and 5-9, we get

$$
S(\gamma)=\frac{\delta^{2} l_{c}}{2 \sqrt{\pi}} \exp \left(-\frac{l_{c}^{2} \gamma^{2}}{4}\right)
$$

For surface roughness without any correlation, all spatial frequencies of roughness are radiated randomly and independently. That means that when we consider the power loss, it is just the sum of the powers radiated by each frequency. Thus,

$$
P_{r a d} \propto \int S(\gamma) d \gamma
$$

Notice, not all frequencies $\gamma$ lead to significant loss. Only those frequencies which satisfy $\beta-k n_{c l}<\gamma<\beta+k n_{c l}$ need to be considered (Ladouceur, 1997). So Eq. 5-11 can be rewritten as 


$$
P_{r a d} \propto \int_{\beta-k n_{c l}}^{\beta+k n_{c l}} S(\gamma) d \gamma
$$

When considering surface correlations, the case will be similar. The magnitude of the radiation is predominantly scattered at an angle $\theta$ relative to the waveguide axis, so we have

$$
\gamma=\beta-k n_{c l} \cos \theta
$$

Summing over all radiation angles, we have

$$
P_{r a d} \propto \int_{0}^{\pi} S\left(\beta-k n_{c l} \cos \theta\right) d \theta
$$

To effectively express the power loss in a waveguide, an attenuation factor $\alpha$ is used. It is defined as

$$
P=P_{0} e^{-\alpha L}
$$

where $\mathrm{L}$ is the waveguide length, $P_{0}$ is the initial power, and $P$ is the power remaining in the waveguide after the light propagates a distance L. When $\alpha \mathrm{L}$ is small, Eq. 5-16 can be rewritten as

$$
\alpha=\frac{1}{L}\left(\frac{P_{0}-P}{P_{0}}\right)
$$

A more detailed analysis for the attenuation factor was given in reference (Ames, et al., 1983)

$$
\alpha=\frac{1}{L} \int_{0}^{\pi} P_{s-r a d} \alpha_{1} d \theta+\frac{1}{L} \int_{0}^{\pi} P_{c-r a d} \alpha_{2} d \theta
$$

where, $P_{s-r a d}$ and $P_{c-\text { rad }}$ are scattering or radiation power to substrate and cladding layers, respectively. Both of them are complicated functions of angle. Thus,

$$
\begin{aligned}
& \alpha=\left(\frac{\delta_{c}^{2} l_{c}}{\lambda^{3}}\right)\left(n_{w}^{2}-n_{c}^{2}\right) E_{c}^{2} \int_{0}^{\pi} \frac{C}{1+\left(\beta-n_{c} k_{0} \cos \theta\right)^{2} l_{c}^{2}} d \theta \\
& +\left(\frac{\delta_{s}^{2} l_{s}}{\lambda^{3}}\right)\left(n_{w}^{2}-n_{s}^{2}\right) E_{s}^{2} \int_{0}^{\pi} \frac{S}{1+\left(\beta-n_{s} k_{0} \cos \theta\right)^{2} l_{s}^{2}} d \theta
\end{aligned}
$$

where $\lambda$ is the wavelength, $l_{c}$ and $l_{s}$ are correlation lengths, $E_{c}$ and $E_{s}$ are electric fields at cladding surface and substrate surface, respectively. $\beta$ is the propagation constant, and $n_{w}, n_{c}$ and $n_{s}$ are indices of waveguide, cladding, and substrate. $\mathrm{C}$ and $\mathrm{S}$ are coefficients related to waveguide indices, angle, wavelength, and waveguide 
thickness. The integrated angle is from 0 to $\pi$. That means total radiated power is the integral of the power carried by each randomly radiated plane wave. Each planer wave corresponds to a certain spatial frequency of roughness, and is radiated in a particular direction.

For a single $\mathrm{TE}_{00}$ mode in a symmetric ( $\mathrm{n}_{\text {cladding }}=\mathrm{n}_{\text {substrate }}$ ) waveguide, Eq. 5-19 can be rewritten as (Jin, et al., 1996 )

$$
\alpha=\frac{1}{\rho^{5} \beta} \frac{V^{2} U^{2} W}{1+W} \int_{0}^{\pi} \frac{\delta^{2} l_{c}}{\pi\left[1+\left(\beta-n_{c} k_{0} \cos \theta\right)^{2} l_{c}^{2}\right]} d \theta
$$

where $V=k \rho\left(n_{1}^{2}-n_{2}^{2}\right)^{1 / 2}=\sqrt{U^{2}+W^{2}}$ is the degree of guidance, $\rho$ is the half width of the waveguide, $U=\rho\left(k^{2} n_{1}^{2}-\beta^{2}\right)^{1 / 2}$, and $W=\rho\left(\beta^{2}-k^{2} n_{2}^{2}\right)^{1 / 2}$. Extend Eq. 5-20 to more general asymmetric waveguides and the result is

$$
\begin{aligned}
& \alpha=\frac{1}{2 \rho_{1}^{5} \beta} \frac{V_{1}^{2} U_{1}^{2} W_{1}}{1+W_{1}} \int_{0}^{\pi} \frac{\delta^{2} l_{c}}{\pi\left[1+\left(\beta-n_{0} k_{0} \cos \theta\right)^{2} l_{c}^{2}\right]} d \theta \\
& +\frac{1}{2 \rho_{2}^{5} \beta} \frac{V_{2}^{2} U_{2}^{2} W_{2}}{1+W_{2}} \int_{0}^{\pi} \frac{\delta^{2} l_{c}}{\pi\left[1+\left(\beta-n_{2} k_{0} \cos \theta\right)^{2} l_{c}^{2}\right]} d \theta
\end{aligned}
$$

Here, $\rho_{1}$ and $\rho_{2}$ correspond to the distance from mode center to each boundary, respectively. The degrees of guidance, $\mathrm{V}_{1}$ and $\mathrm{V}_{2}$, and the $\mathrm{U}$ and $\mathrm{W}$ expressions become:

$$
\begin{array}{lll}
V_{1}=k \rho_{1} \sqrt{n_{1}^{2}-n_{0}^{2}} & U_{1}=\rho_{1}\left(k^{2} n_{1}^{2}-\beta^{2}\right)^{1 / 2} & W_{1}=\rho_{1}\left(\beta^{2}-k^{2} n_{0}^{2}\right)^{1 / 2} \\
V_{2}=k \rho_{2} \sqrt{n_{1}^{2}-n_{2}^{2}} & U_{2}=\rho_{2}\left(k^{2} n_{1}^{2}-\beta^{2}\right)^{1 / 2} & W_{2}=\rho_{2}\left(\beta^{2}-k^{2} n_{2}^{2}\right)^{1 / 2}
\end{array}
$$

For a single alumina layer, the program in Appendix I was used to compute the theoretical scattering loss due to surface roughness. The following set of parameters were used: $\mathrm{n}_{0}=1, \mathrm{n}_{1}=1.659, \mathrm{n}_{2}=1.4701, \mathrm{~d}=144 \mathrm{~nm}, \lambda=632.8 \mathrm{~nm}, \delta=1 \mathrm{~nm}$, and $l_{c}$ $=100 \mathrm{~nm}$. The effective index is 1.4736. After all these numbers are input into the program, the resulting attenuation factor was found to be $\alpha=0.87 \mathrm{~dB} / \mathrm{cm}^{-1}$. Normally, a higher attenuation is observed experimentally. Since this theoretical value is less than the experimental value, there must be additional factors leading to scattering losses in addition to waveguide surface roughness.

For a correlation length of $100 \mathrm{~nm}$, Fig. 5.4 shows how the scattering loss depends on surface roughness in a single alumina waveguide (for air cladding). As the 
surface roughness increases, the scattering loss increases significantly. Figure 5.5 shows the relationship between correlation length and scattering loss for the same waveguide structure.

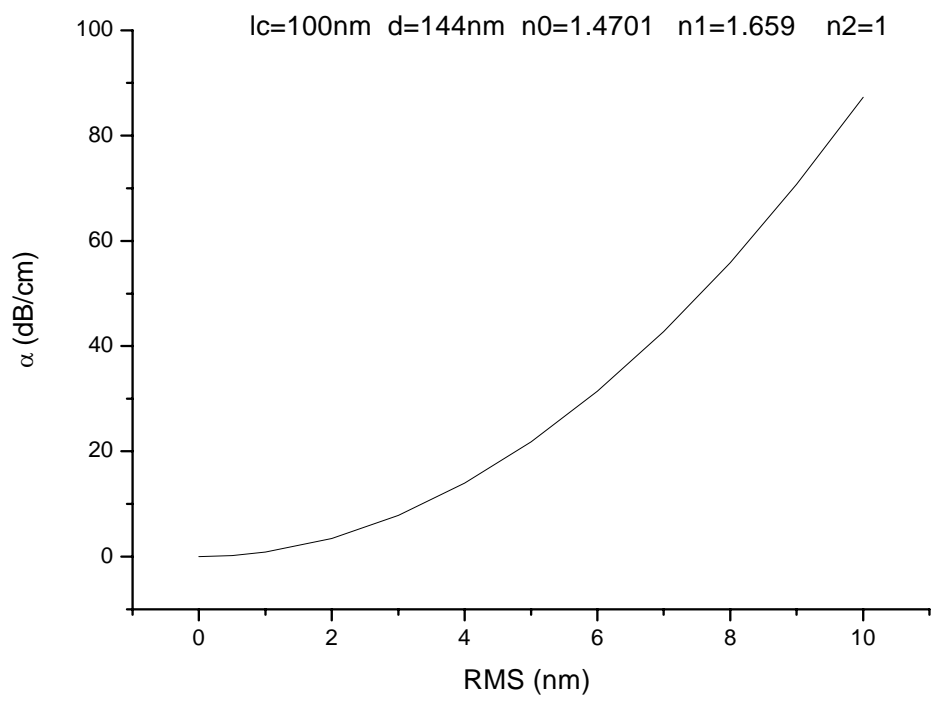

Fig. 5.4. Scattering loss vs. RMS roughness in an alumina waveguide.

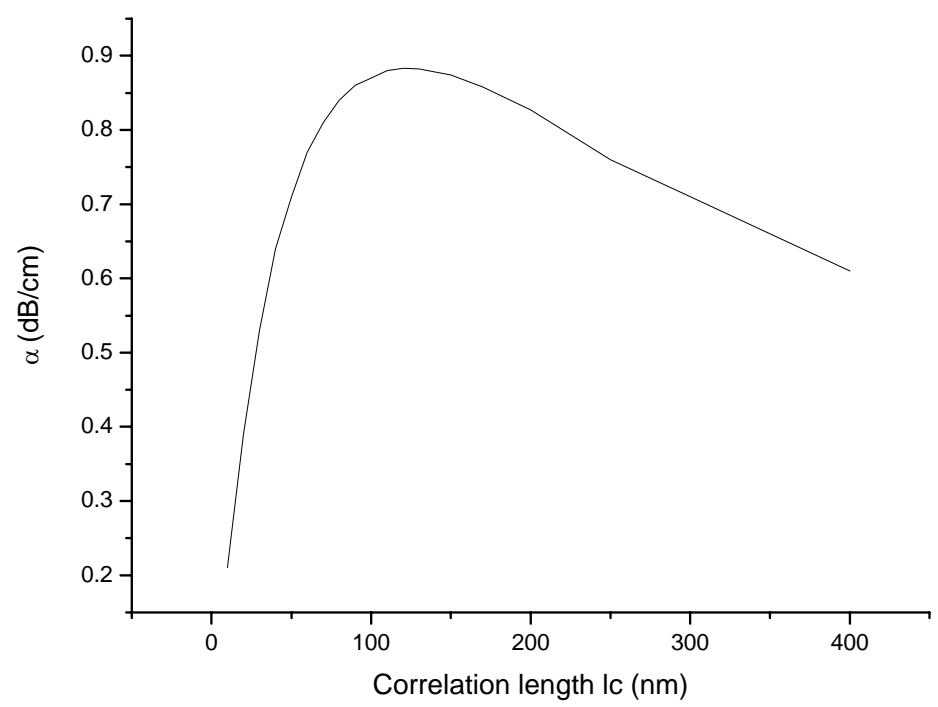

Fig. 5.5. Scattering loss vs. correlation length. 
The scattering loss will have a maximum value when the correlation length approaches a certain number. From Fig. 5.5, the value of $L_{\max }$ is approximately $120 \mathrm{~nm}$. In different cases, this number will be different. An approximation method to get the maximum correlation length can be found in the literature (Huang, 1994).

$$
L_{\max } \approx \frac{1}{\beta-n_{\text {cladding(substrate) }} k_{0}}
$$

This is very a rough approximation. Using Eq. 5-18 for our case, we get a value of $L_{\max } \sim 560 \mathrm{~nm}$ in such a single waveguide.

Much more complicated mathematics has to be used when considering coupled waveguides. Figure 5.6 depicts a coupled waveguide structure. The surface roughness may be caused by the deposition process. Only a single evanescent mode is present in each waveguide and they are physically isolated from each other. In addition, the refractive index of each waveguide can vary along the longitudinal direction. Both surface roughness and index fluctuations can be treated as slight

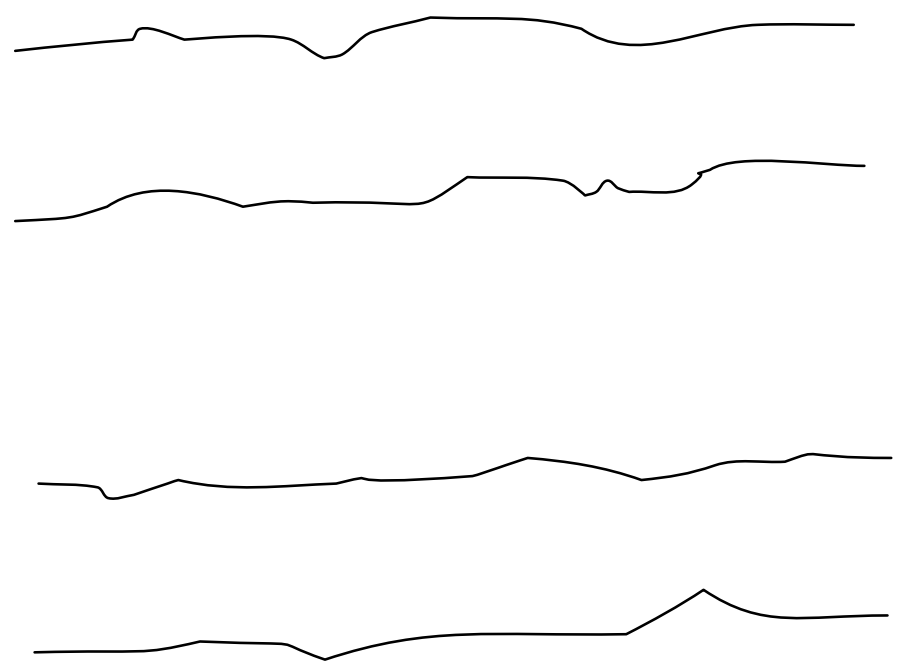

Fig. 5.6. Coupled waveguides with inhomogeneous surfaces.

perturbations of a uniform coupler, as mentioned in Chapter 2. Both perturbations contribute to variations in coupling coefficient. Therefore, the standard coupled mode equations can be written as 


$$
\begin{aligned}
& \frac{\partial \widetilde{a}_{1}}{\partial z}+i C_{11} \widetilde{a}_{1}+i C_{11}^{\prime} \widetilde{a}_{1}+i C_{12} \widetilde{a}_{2} e^{i\left(\widetilde{\beta}_{1}-\widetilde{\beta}_{2}\right)}+i C_{12}^{\prime} \widetilde{a}_{2} e^{i\left(\widetilde{\beta}_{1}-\widetilde{\beta}_{2}\right)}=0 \\
& \frac{\partial \widetilde{a}_{2}}{\partial z}+i C_{22} \widetilde{a}_{2}+i C_{22}^{\prime} \widetilde{a}_{2}+i C_{21} \widetilde{a}_{1} e^{i\left(\widetilde{\beta}_{2}-\widetilde{\beta}_{1}\right)}+i C_{21}^{\prime} \widetilde{a}_{1} e^{i\left(\widetilde{\beta}_{2}-\widetilde{\beta}_{1}\right)}=0
\end{aligned}
$$

where the symbol $\sim$ means an average quantity is used. $C_{11}^{\prime}, C_{22}^{\prime}, C_{12}^{\prime}$ and $C_{21}^{\prime}$ are perturbation terms of coupling coefficients. These perturbations are produced by either surface imperfection or refractive index inhomogeneities. Although Eq.. 5-23 gives the accurate coupled mode equation under a perturbation, it is too complicated to get an analytical solution. An approximation or assumption has to be considered. For the weakly guiding condition, one can say that $\frac{\left(n_{\text {waveguide }}-n_{\text {cladding }}\right)}{n_{\text {waveguide }}} \times 100 \%<1 \%$. For this case, an approximation method has been described (Ladouceur, et al., 1995). When two waveguides are identical, just simply use $C$ ' to express these variations and rewrite the coupled mode expression of Eq. 2-74 as

$$
\begin{aligned}
& \frac{\partial a_{1}}{\partial z}=-i C_{11} a_{1}+i C_{11}^{\prime} a_{1}-i C a_{2} \\
& \frac{\partial a_{2}}{\partial z}=-i C_{22} a_{2}-i C_{22}^{\prime} a_{2}-i C a_{1}
\end{aligned}
$$

where $C_{11}{ }^{\prime}$ and $C_{22}{ }^{\prime}$ are z-dependent perturbation terms which represent coupled coefficients having small variations. In symmetric coupled waveguides, these terms will be equal in value, i.e., $C^{\prime}=C_{11}{ }^{\prime}=C_{22}{ }^{\prime}$. The terms $C_{11}$ and $C_{22}$ are self coupling coefficients which are small in numerical value compared to coupling coefficient $\mathrm{C}$. In addition, $C^{\prime}$ satisfies the following conditions.

$$
\begin{aligned}
& <C^{\prime}>=0 \\
& <C^{\prime}(z) C^{\prime}(z+u)>=\delta^{2} \exp \left(-\frac{|u|}{l_{c}}\right) \\
& <\frac{d C^{\prime}}{d z}>=0 \\
& <\frac{d^{2} C^{\prime}}{d z^{2}}>=0
\end{aligned}
$$


Equation 5-24 can be written as

$$
\left(L_{0}+L_{1}\right) a(z)=0
$$

where

$$
L_{0}=\left(\begin{array}{cc}
\frac{\partial}{\partial z} & -i C \\
-i C & \frac{\partial}{\partial z}
\end{array}\right) \quad L_{1}=\left(\begin{array}{cc}
-i C^{\prime} & 0 \\
0 & i C^{\prime}
\end{array}\right) \quad a(z)=\left(\begin{array}{l}
a_{1} \\
a_{2}
\end{array}\right)
$$

Upon averaging both sides, we have

$$
\left\langle\left(L_{0}+L_{1}\right)\right\rangle\langle a(z)\rangle=0
$$

and

$$
\left[\left\langle L_{0}\right\rangle+\left\langle L_{1}\right\rangle+\left\langle L_{1}\right\rangle L_{0}^{-1}\left\langle L_{1}\right\rangle-\left\langle L_{1} L_{0}^{-1} L_{1}\right\rangle\right]\langle a(z)\rangle=0
$$

Since $\left\langle L_{0}\right\rangle=L_{0},\left\langle L_{1}\right\rangle=0$, the above equation can be written as

$$
\left[L_{0}-\left\langle L_{1} L_{0}^{-1} L_{1}\right\rangle\right]\langle a(z)\rangle=0
$$

Appling Green's function to above equation and performing some mathematical steps, the approximate final results become (Ladouceur, et al., 1995)

$$
C^{\prime}=C \frac{1+l_{c}^{2} \delta^{2}}{1-l_{c}^{2} \delta^{2}}
$$

and

$$
\alpha=\frac{2 l_{c} \delta^{2}}{1-l_{c}^{2} \delta^{2}}
$$

where $\mathrm{C}$ is the coupling coefficient of a uniform waveguide, $\mathrm{C}^{\prime}$ is the modulated coupling coefficient, and $\alpha$ is the total power attenuation factor. From this equation, the surface roughness changes the coupling length, and introduces the power scattering loss. This equation shows that power scattering loss only depends on how rough the surface is as well as the correlation length of surface roughness. This is suitable for the weakly guiding case in which the index of the waveguide is just slightly larger than the index of the cladding (recall that the percent difference is smaller than 1\%). However, when refractive index differences between waveguide and cladding (or substrate) is larger than $1 \%$, this equation is no longer valid. For the SPARROW biosensor waveguide, with the 
particular choice of parameters $l_{c}=100 \mathrm{~nm}$ and $\delta=1 \mathrm{~nm}$, Eq. 5-32 gives the attenuation factor as $\alpha=1 * 10^{-27} \mathrm{~dB} / \mathrm{cm}$. In addition, the new coupling length $C^{\prime} \rightarrow C$. These results imply that surface roughness doesn't affect anything in coupled waveguides at all. This conclusion obviously doesn't agree with the actual case of real devices.

For a large refractive index contrast, a mathematical derivation of an exact solution seems impossible because of too many unknown parameters. However, an approximation method can be applied in such case. Using Eq. 5-21, the scattering factor in a single waveguide can be easily obtained. Assuming the two waveguides are not too close and the scattering in each waveguide doesn't affect each other, the total power scattering can be considered as the sum of two contributions and can be written as

$$
P_{\text {scattering }}=\int P_{1}(\mathrm{z}) \alpha_{1} d z+\int P_{2}(\mathrm{z}) \alpha_{2} d z
$$

where $P_{1}$ is the power in the first waveguide, $P_{2}$ is the power in the second waveguide, $\alpha_{1}$ and $\alpha_{2}$ are loss coefficients, and $L$ is the total length of the waveguide. Since power in coupled waveguides exchanges periodically, both $P_{1}$ and $P_{2}$ depend on position z. For synchronous coupled waveguides (see section 2-2-2), we have

$$
\begin{aligned}
& P_{1}=P_{0} \cos ^{2}(\mathrm{Cz}) \\
& P_{2}=P_{0} \sin ^{2}(\mathrm{Cz})
\end{aligned}
$$

If coupled waveguides are asynchronous, we have another equation to evaluate the power in each waveguide (see section 2-2-3)

$$
\begin{aligned}
& \left|a_{1}(z)\right|^{2}=P_{1}=1-\eta \sin ^{2}\left(\frac{\pi}{2 L_{c}} z\right) \\
& \left|a_{2}(z)\right|^{2} \frac{\beta_{1}}{\beta_{2}}=\frac{\beta_{1}}{\beta_{2}} P_{2}=\eta \sin ^{2}\left(\frac{\pi}{2 L_{c}} z\right)
\end{aligned}
$$

Combining Eqs. 5-33 and 5-34 or 5-35, the power loss fraction can be obtained.

For the transducer structure in the SPARROW biosensor (see Fig 5.7), assume a total length of waveguide of $1 \mathrm{~mm}$, an RMS roughness of $1 \mathrm{~nm}$, surface correlation length is $60 \mathrm{~nm}$ (see Fig. 5.8) and all other parameters come from Fig. 5.7. 
Water $: n=1.3318$

\begin{tabular}{c}
\hline Waveguide $1 \mathrm{n}=1.659 \mathrm{~d}=194 \mathrm{~nm}$ \\
\hline $\mathrm{SiO}_{2}$ layer $\boldsymbol{n}=1.457 \mathrm{~d}=1100 \mathrm{~nm}$ \\
\hline Waveguide $2 \mathrm{n}=1.659 \mathrm{~d}=144 \mathrm{~nm}$ \\
\hline
\end{tabular}

Borofloat Substrate: $\boldsymbol{n}=1.4701$

Fig . 5.7. A model for transducer structure

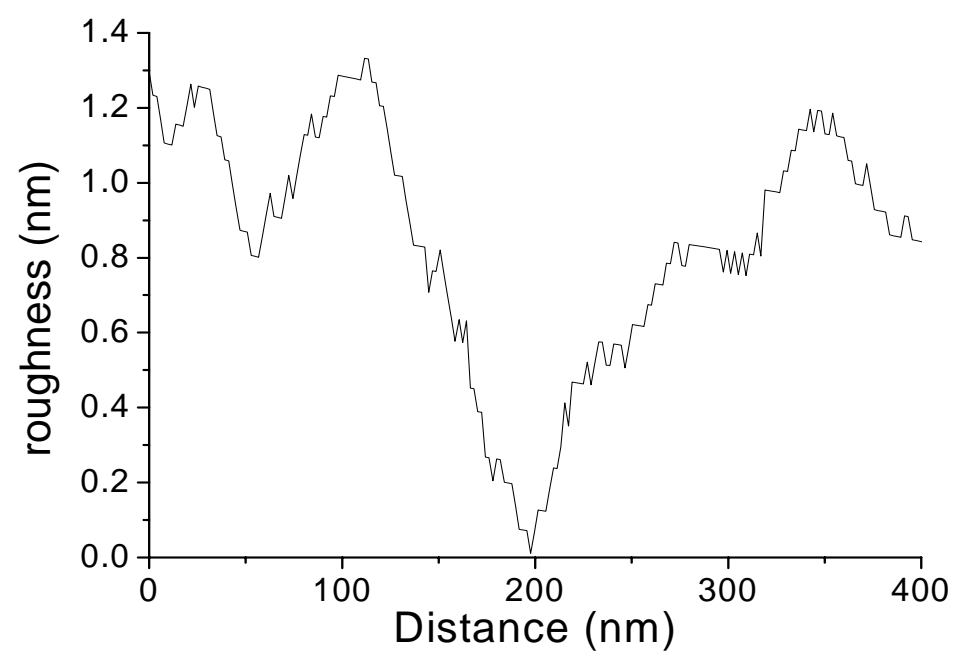

Fig. 5.8. Profile of alumina waveguide surface roughness ( $l_{c}$ is estimated to be $60 \mathrm{~nm}$ from this graph)

For the upper waveguide in the SPARROW biosensor, the loss coefficient is $0.325 \mathrm{~dB} / \mathrm{cm}$. For the buried waveguide, the loss coefficient is $0.196 \mathrm{~dB} / \mathrm{cm}$. Thus, according to Eq. 533, the final scattering loss is $0.0017 P_{0}$. That means 0.17 percent power loss after $1 \mathrm{~mm}$ transmission. Figure 5.9 shows the power loss fraction in a 1-mm length vs. the RMS surface roughness for coupled waveguides. 


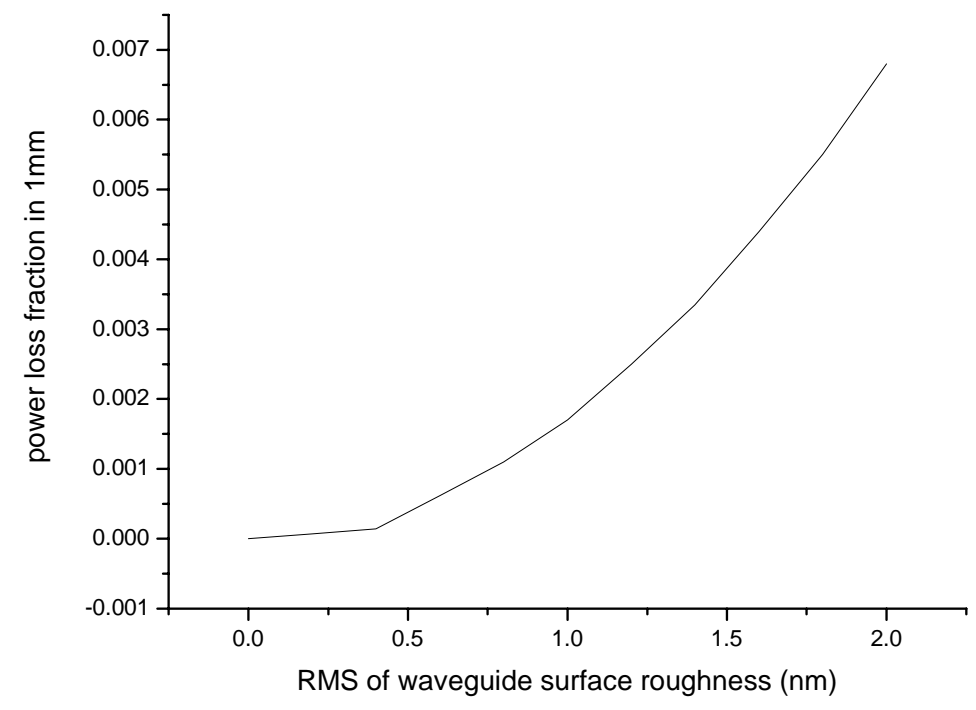

Fig. 5.9. Power loss fraction in coupled waveguides vs. RMS roughness

\section{5-2 Random surface loading}

When the biosensor is in operation, the biolayer will capture the specific molecule in a target solution. These binding events are randomly located on the biosensor surface (see Fig. 5.10) and result in changes of the output power. By monitoring this power variation, we can detect a specific bio-agent. However, how this change relates to percent coverage of the molecule has not yet been addressed. In this section, we will explore the effect of this random binding and link it to surface loading. 
Binding molecule in random position

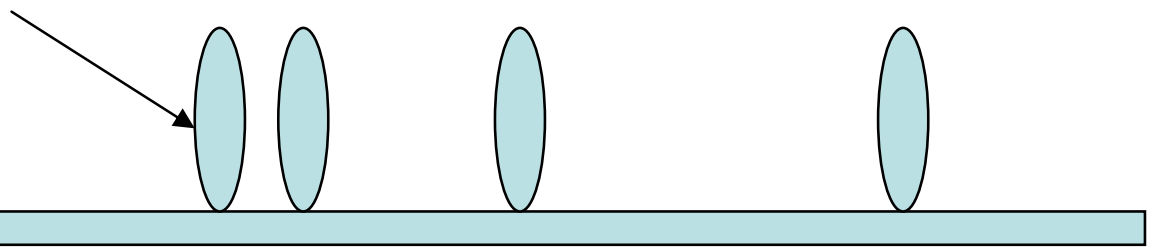

Fig. 5.10 Random binding events on biosensor's surface.

First, a brief review will be given on how other authors (Rigrod, et al., 1976; Marcuse, 1990; Hall, 1990; Griffel, et al., 1991; Huang, et al., 1991; West, et al., 1994; Passaro, 1995; Little, 1996; Garcia-martin, 2005) deal with the surface roughness and grating problems in this case. Generally, the coupled mode equation can be divided into a uniform part and a perturbation part. In the uniform part, an average thickness was used to calculate the average propagation constant. However, if the size of particles is too big (e.g. diameter of molecule is larger than penetration depth), an average thickness is obviously not suitable. To get the average effective refractive index of the top waveguide and an average propagation constant, we have to find a new way to solve this problem. An equivalent layer was considered to substitute for the random binding events layer. The height of the equivalent layer is the same as the binding events height. The refractive index of this layer is independent of the actual spatial distribution of the binding molecules, and is only related to percent coverage. A depiction of the equivalent layer is shown in Fig. 5.11. This approach greatly simplifies the propagation constant problem. 


\section{Equivalent layer}

Fig. 5.11. Depiction of the equivalent layer on top of waveguides.

Physically, this means that we take an average of the permittivity in this region. The refractive index of the equivalent layer can be expressed as

$$
n_{\text {eq }}=\sqrt{n_{\text {molecule }}^{2} * \operatorname{cov} \text { erage }+n_{\text {water }}^{2} *(1-\operatorname{cov} \text { erage })}
$$

Thus, the propagation constant of the upper waveguide can be obtained. For a buried waveguide, the propagation constant doesn't change. Directly from these two propagation constants, the coupling length and coupling efficiency can be obtained. To verify this equivalent-layer approach, the structure shown in Fig. 5.12 was used, and the results of numerical calculations and computer simulations are compared.

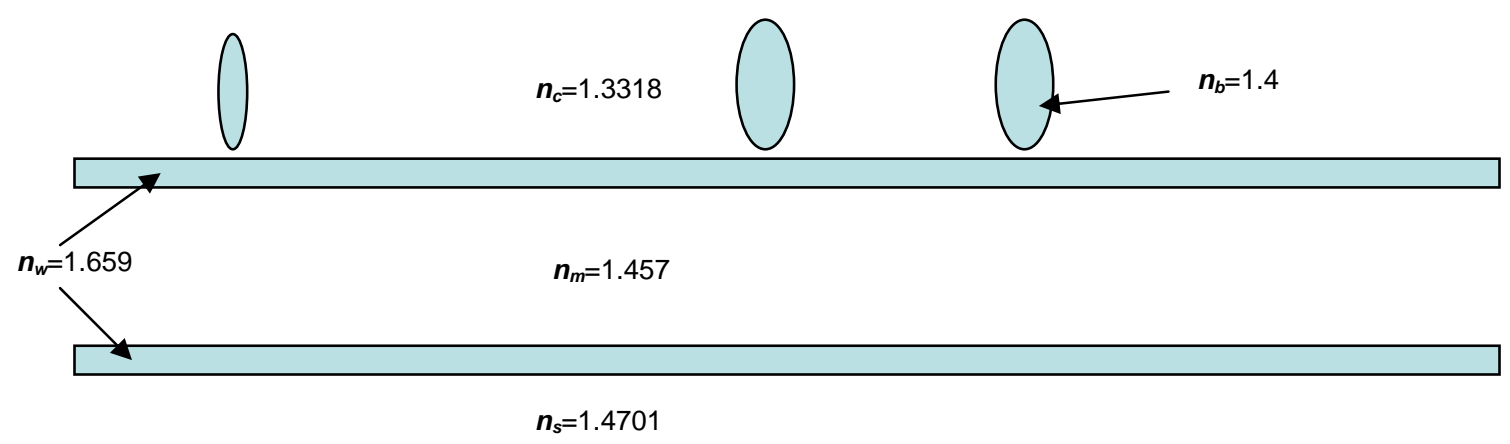

Fig. 5.12. Numerical model of biosensor with random binding molecule.

The height of the binding events is taken to be $2000 \mathrm{~nm}$ and the percent coverage is $2 \%$. By mathematical calculation, the numerical results for coupling length and coupling efficiency for the above structure are 


$$
L_{c}=184 n m \text { and } \eta=96.9 \%
$$

In the case of no binding events, the coupling length and coupling efficiency are $L_{c}=187 \mathrm{~nm}$ and $\eta=99 \%$, respectively. This shows that only a very small change is introduced by binding. Computer simulation results also supported this conclusion. From the simulation program, the results shown in Fig. 5.13 were obtained. It is difficult to determine the numerical coupling efficiency from the graph, but the coupling length is the same as that obtained from the calculations.

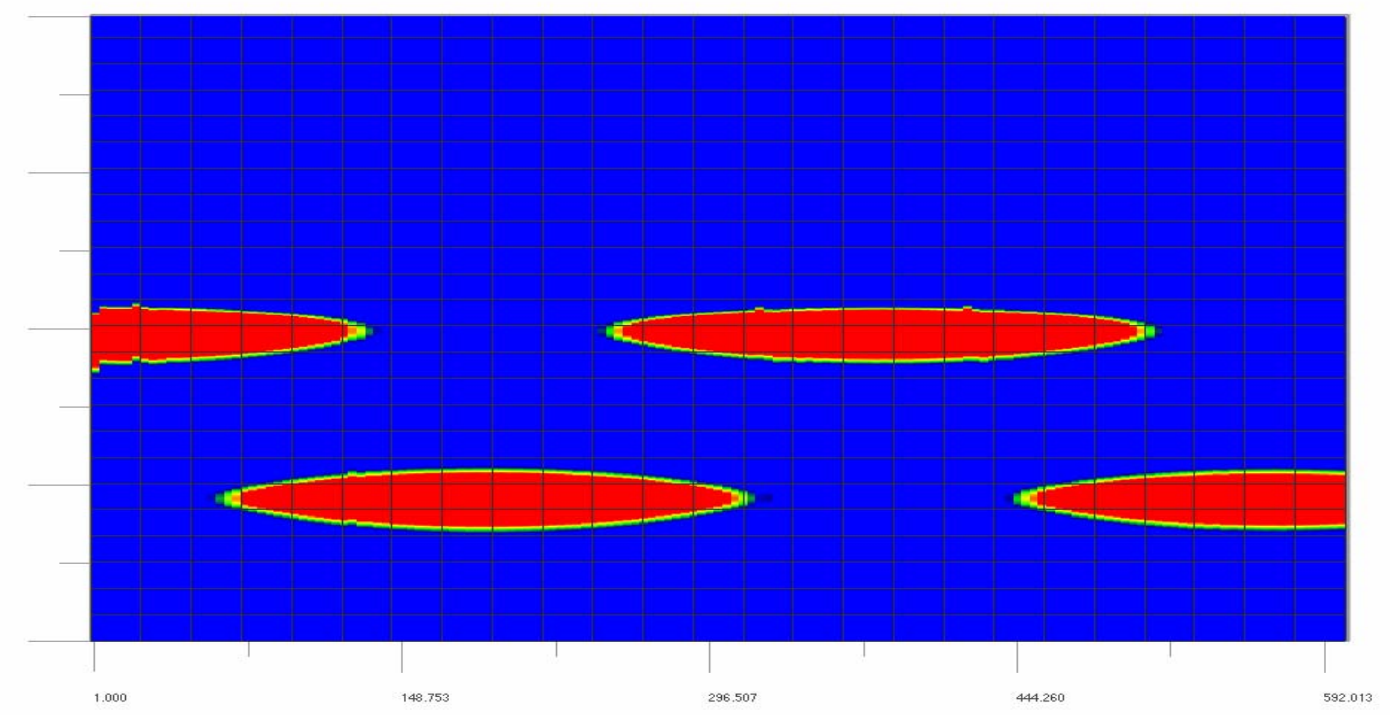

Fig. 5.13. BPM-modified coupling length and coupling coefficiency for a surface coverage of $2 \%$.

The effect of larger surface coverage was explored. Keeping the same settings and just changing the coverage from $2 \%$ to $20 \%$, the numerical calculations gave reduced values for the coupling length and coupling efficiency of

$$
L_{c}=136 \mathrm{~nm} \text { and } \eta=54.1 \%
$$

The computer simulation resulted in the graph shown in Fig. 5.14. The coupling length obtained from the simulation has the same value as that obtained from theoretical calculation. For a particular coupling length, coupling coefficient, and waveguide length, the next step is to quantitatively predict the output power. 


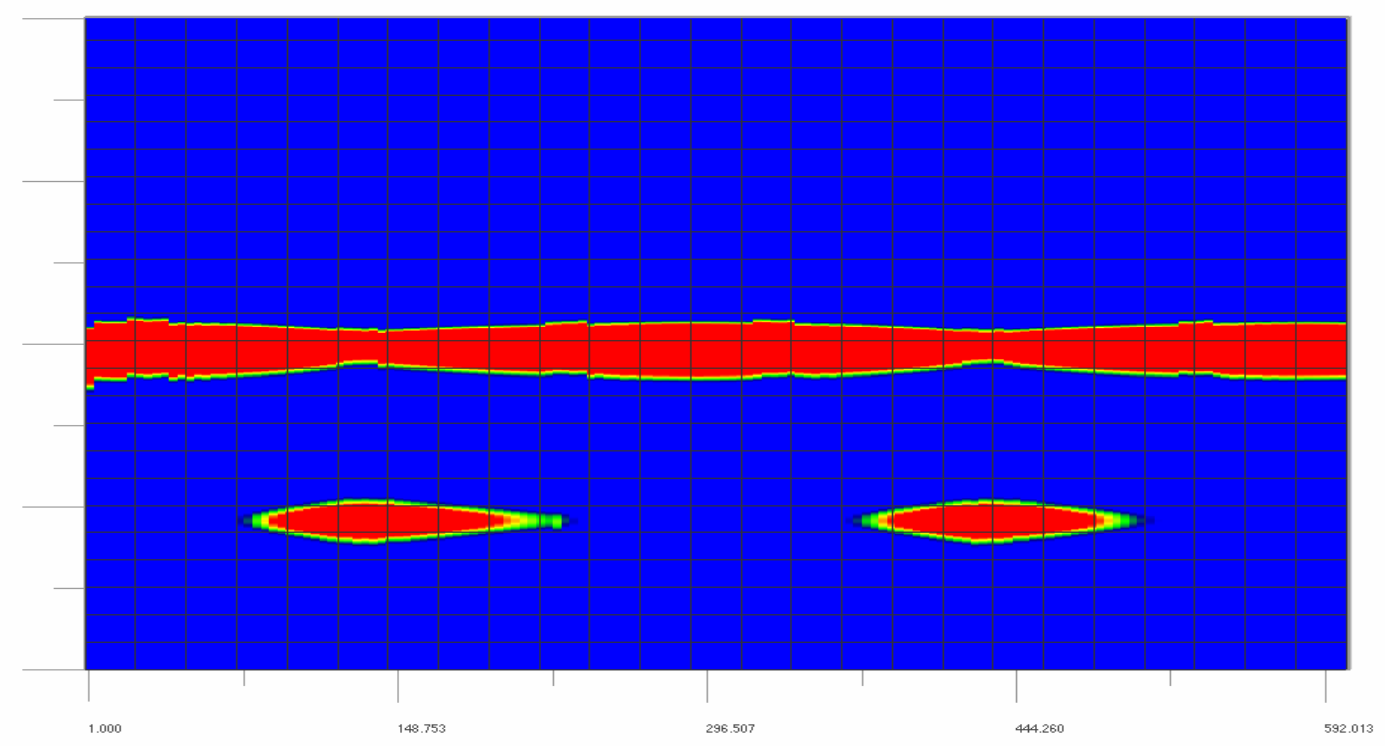

\section{Fig. 5.14. BPM-modified coupling length and coupling coefficiency for a surface coverage of $20 \%$.}

According to the previous discussion, both surface roughness and the random binding of molecules affect the coupling length, coupling efficiency, and scattering loss. Thus, in order to predict output power accurately, we have to explore how much scattering loss is caused by these binding molecules. Obviously, different spatial distributions of these molecules will lead to different amounts of scattering. For example, for a certain percent coverage, there will be the least amount of scattering if all the molecules assemble together. The exact solution is impossible to obtain unless we know the exact surface function. However, if the molecular binding is totally random, they will exhibit a correlated behavior, somewhat like surface roughness. By a statistical method, it is possible to then evaluate the scattering loss.

In section 5-1, the perturbation method was used to derive the scattering loss. This method is applicable if the surface RMS roughness and correlation length are less than the wavelength of HeNe laser $(633 \mathrm{~nm})$. However, the binding events which we used in experiments (see section 4-3-4) have a rather large size and large correlation length. There will be a huge error if we still use the perturbation method. Theoretically, we can achieve small correlation lengths with high percent coverage, but experimentally, it has proven to be very difficult to get a high percent of coverage. Also, a high percent 
of coverage has a large detuning factor that causes the coupling coefficient to drop to 0 . Another method, called the volume current method (VCM) was then applied in this dissertation work.

Generally, the VCM is used to calculate the scattering radiation from small refractive index perturbations in an otherwise uniform waveguide. When an evanescent wave propagates in the waveguide, the index inhomogeneities generate localized effective volume polarized currents which leads to scattering of radiation. The accuracy of VCM depends on the magnitude of the perturbing elements. In the modeling of the SPARROW biosensor, we are using an equivalent layer to substitute for these binding events and a cladding solution in this layer region. For large index contrast of binding events and cladding solution in this region, the accuracy of results obtained using VCM will become less certain. So far, there is no analytic expression which can solve this problem, unless the binding events are periodic. However, if the index contrast is not too large (refractive index of binding events is less than effective index of top waveguide), VCM offers a good approach to simulate biosensor behavior.

To simplify the problem, first we simulate these binding events as rectangular beads. If the diameter of a bead is less than the penetration depth, the scattering loss will exhibit a complex behavior which has not been addressed in this work and will be the subject of future studies. In this case, we assume the width and height of rectangular beads equal to the diameter of bead. If the diameter is larger than penetration depth, the effective width of these rectangular beads will depend on the diameter of the microbeads and the effective height will be the penetration depth as shown in Fig. 5.15. As mentioned in chapter 2, this penetration depth is the distance where the total field magnitude drops to $1 /$ e. Beyond the penetration distance above the surface there is little scattering loss, however we do take this contribution to scattering to be taken into account by consideration of the full rectangle as an area of interaction rather than neglecting the arced edge areas where no bead area intersects. 


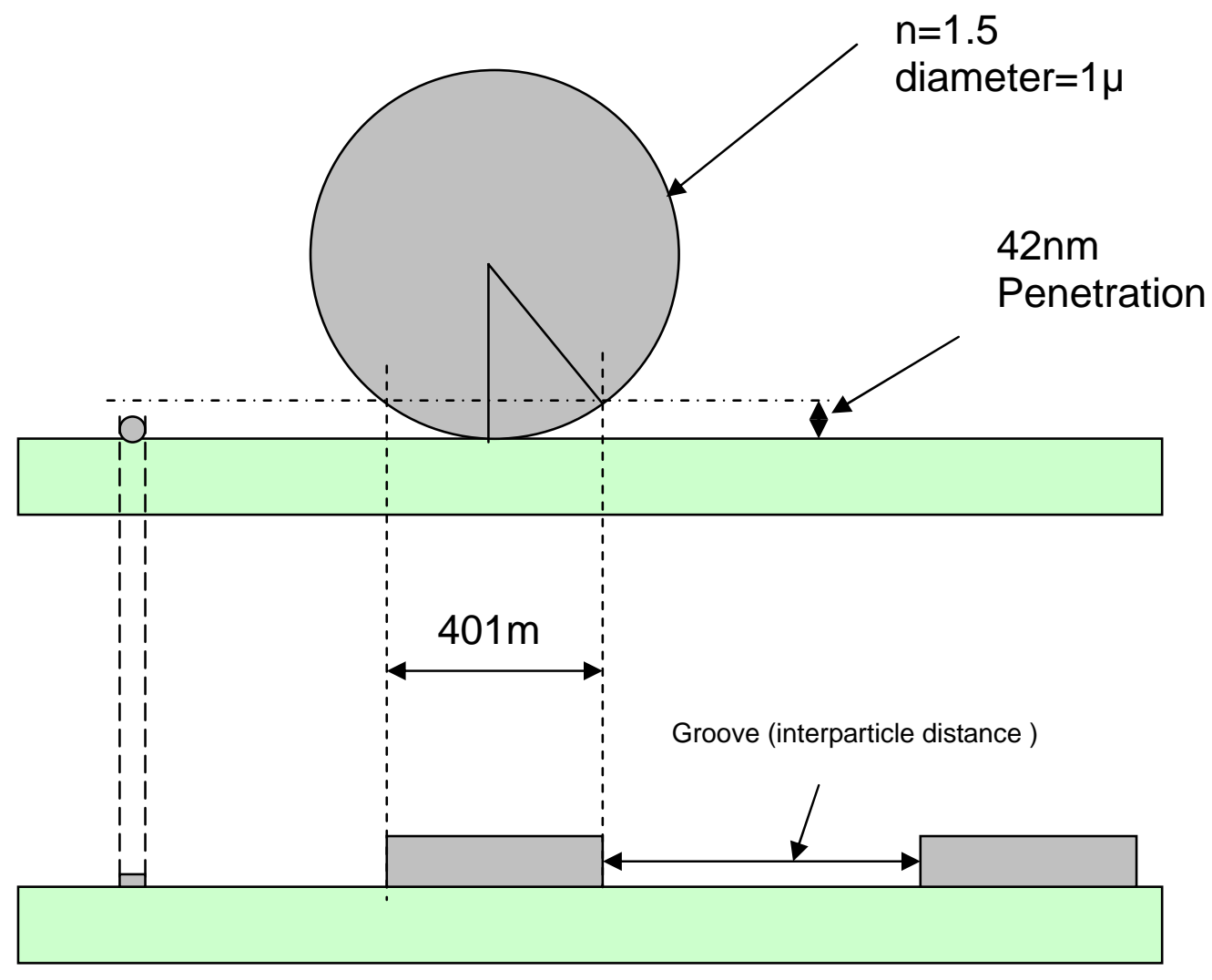

Fig. 5.15. Method to simplify microbeads attachment

If the permittivity of the cladding solution and binding events are $\varepsilon_{1}$ and $\varepsilon_{2}$ respectively, following VCM theory, the total dielectric constant in that binding layer can be written as

$$
\varepsilon(z)=\varepsilon_{2}+\delta \varepsilon(z)
$$

The expression for the effective dielectric perturbation is

$$
\delta \varepsilon(z)=\left(\varepsilon_{1}-\varepsilon_{2}\right) f(z)
$$

where

$$
\begin{array}{ll}
f(z)=1 & \text { in the volume of a groove } \\
f(z)=0 & \text { otherwise }
\end{array}
$$

The induced polarized current can be written as

$$
\vec{J}_{p}=i w\left(\varepsilon_{1}-\varepsilon_{2}\right) f(z) E(z)
$$


where $E(z)$ is the electric field in a uniform waveguide. According to standard electromagnetic theory, the electric and magnetic fields can be expressed in terms of a vector potential $\vec{A}$.

$$
\begin{aligned}
\vec{B} & =\vec{\nabla} \times \vec{A} \\
\vec{E} & =-\vec{\nabla} \phi+i w \vec{A}
\end{aligned}
$$

Thus,

$$
\left(\nabla^{2}+\omega^{2} \mu_{0} \varepsilon_{2}\right) \vec{A}=\mu_{0} \vec{J}_{p}
$$

where

$$
\vec{A}=\mu_{0} \int G\left(\vec{r}-\vec{r}^{\prime}\right) \vec{J}_{p} d^{3} r^{\prime}
$$

Here, $G\left(\vec{r}-\vec{r}^{\prime}\right)$ is the Green function for the two-dimensional Helmholtz equation.

$$
G\left(\vec{r}-\vec{r}^{\prime}\right)=\frac{i}{4} H\left(k \mid \vec{r}-\vec{r}^{\prime}\right)
$$

In Eq. $5-43, \mathrm{H}$ is the zero ${ }^{\text {th }}$-order Hankel function of the first kind (also referred to as Bessel functions of the third kind). Normally, for a periodic waveguide grating, the nonzero component of the vector potential in the far field is given by

$$
\vec{A}=\frac{\mu_{0} \omega\left(\varepsilon_{1}-\varepsilon_{2}\right) E_{0}}{4 \cos (\kappa h / 2)} \sqrt{\frac{2}{\pi k r}} e^{-i \pi / 4} e^{i k r} \int_{\text {grooves }} \cos (\kappa x) e^{-i k \hat{r} \cdot r^{\prime}} e^{i \beta z^{\prime}} d^{2} r^{\prime}
$$

Correspondingly, the radiation power can be computed from the Poynting vector

$$
\vec{S}=\frac{1}{2} \vec{E} \times \vec{H}=\frac{\omega^{2}}{2} \sqrt{\frac{\varepsilon_{2}}{\mu_{0}}}|\hat{r} \times \vec{A}|^{2} \hat{r}=\frac{\mu_{0} \omega^{3} \varepsilon_{0}^{2}\left(\varepsilon_{1}-\varepsilon_{2}\right)^{2} E_{0}{ }^{2}}{16 \pi \cos ^{2}(\kappa h / 2) r}|A|^{2}|E|^{2}|D|^{2} \hat{r}
$$

where $\varepsilon$ is permittivity, $\kappa$ is wavevector component in $\mathrm{x}$ direction. The parameters $\mathrm{A}, \mathrm{E}$, and D in Eq. 5-45 are obtained from

$$
\begin{aligned}
& A=\sum_{n=0}^{N-1}\left(e^{i \Lambda(\beta-k \sin \theta)}\right)^{n} \\
& E=\int_{0}^{w_{1}} e^{i z^{\prime}(\beta-k \sin \theta)} d z^{\prime} \\
& D=\int_{d}^{h+d} \cos \left(\kappa x^{\prime}\right) e^{i k x^{\prime} \cos \theta} d x^{\prime}
\end{aligned}
$$

where $N$ is number of particles, $\Lambda$ is grating period. $h$ is height of groove. $w_{1}$ is width of groove. $d$ is the waveguide thickness. So, the fractional loss per period of a 
propagation mode under the grating is given by

$$
F=\frac{d u_{0} / d t}{u_{0}} T
$$

where $u_{0}$ is the energy in the waveguide mode under the grating region. Further derivation gives the power loss by the grating as

$$
F=\frac{\left(\varepsilon_{1}-\varepsilon_{2}\right)^{2} k^{2}}{2 \varepsilon_{1} \varepsilon_{2} N \Lambda h\left[1+\frac{\sin (\kappa h)}{\kappa h}\right]} \int_{0}^{2 \pi}|A|^{2}|E|^{2}|D|^{2} d \theta
$$

However, Eq. 5-48 is only suitable for small particles scattering because the variation of effective propagation constant $\Delta \beta_{\text {eff }}$ is required to be small. In our case, the binding molecules have large size. If the index of the binding molecule is larger than the system effective index, $\Delta \beta_{\text {eff }}$ will be large from point to point. At this case, the results from Eq. 5-48 will be far from accurate results. Experimentally, the latex beads were used (see section 4-3-4). The refractive index of the latex beads is assumed to 1.5, which is less than the effective index of the system. Under this condition, $\Delta \beta_{\text {eff }}$ should be small enough. Thus, an equivalent layer can be built and the electric field in this layer will attenuate $\left(E=e^{-\alpha x}\right)$. Similar to the grating case, by substitute this electric field to Eq. 542 , we have a new vector potential, which is written as

$$
\vec{A}=\frac{\mu_{0} \omega\left(\varepsilon_{1}-\varepsilon_{2}\right) E_{0}}{4} \sqrt{\frac{2}{\pi k r}} e^{-i \pi / 4} e^{i k r} e^{\alpha h / 2} \int_{\text {grooves }} e^{-\alpha x^{\prime}} e^{-i k \hat{r} \cdot r^{\prime}} e^{i \beta z^{\prime}} d^{2} r^{\prime}
$$

where

$$
\hat{r} \cdot \hat{r}^{\prime}=x^{\prime} \cos \theta+Z^{\prime} \sin \theta
$$

The integral in Eq. 5-50 can be written as

$$
\int_{\text {grooves }} e^{-\alpha x^{\prime}} e^{-i k \hat{r} \hat{r} \hat{r}^{\prime}} e^{i \beta z^{\prime}} d^{2} r^{\prime}=\int_{h / 2-d_{1}}^{h / 2} e^{-\alpha x^{\prime}-i k x^{\prime} \cos \theta} d x^{\prime} \int_{\text {grooves }} e^{i z^{\prime}(\beta-k \sin \theta)} d z^{\prime}
$$

The first integral on the right-hand side of Eq. 5-51 can be written as

$$
D=\int_{d}^{h+d} e^{-\alpha x^{\prime}-i k x^{\prime} \cos \theta} d x^{\prime}=\frac{1}{-\alpha-i k \cos \theta}\left(e^{(-\alpha-i k \cos \theta) h / 2}-e^{(-\alpha-i k \cos \theta)\left(h / 2-d_{1}\right)}\right)
$$


To solve the second integral part, the spatial distribution of random binding events has to be known. Assuming the binding molecules are periodically located on the biolayer surface, i.e., like a grating, and the period is $\Lambda$, this integral can be expressed as

$$
\int_{\text {grooves }} e^{i z^{\prime}(\beta-k \sin \theta)} d z^{\prime}=\sum_{n=0}^{N-1}\left(e^{i \Lambda(\beta-k \sin \theta)}\right)^{n} \int_{0}^{w_{1}} e^{i z^{\prime}(\beta-k \sin \theta)} d z^{\prime}
$$

Therefore,

$$
\vec{S}=\frac{\mu_{0} \omega^{3} \varepsilon_{0}^{2}\left(\varepsilon_{1}-\varepsilon_{2}\right)^{2} E_{0}{ }^{2}}{16 \pi r} e^{\alpha h}|A|^{2}|E|^{2}|D|^{2} \hat{r}
$$

According to Eq. 5-45 with $N=2$, the power loss between two particles can be evaluated. This fraction can be expressed as

$$
F=\frac{\left(\varepsilon_{1}-\varepsilon_{2}\right)^{2} k^{2}}{4 \varepsilon_{1} \varepsilon_{2} \Lambda h\left[1+e^{\alpha h} / \alpha h\right]} \int_{0}^{2 \pi}|A|^{2}|E|^{2}|D|^{2} d \theta
$$

Varying the period $\Lambda$ and particle width $w_{1}$, the power loss due to any two particles will be obtained. For a given percent coverage, the number of particles and the average distance $d_{\text {ave }}$ between any two isolated particles are known. Fig. 5.16 shows the scattering loss coefficient for different interparticle distance. Here, we assume the diameter of beads is $1 \mu \mathrm{m}$ and the refractive index is 1.5 .

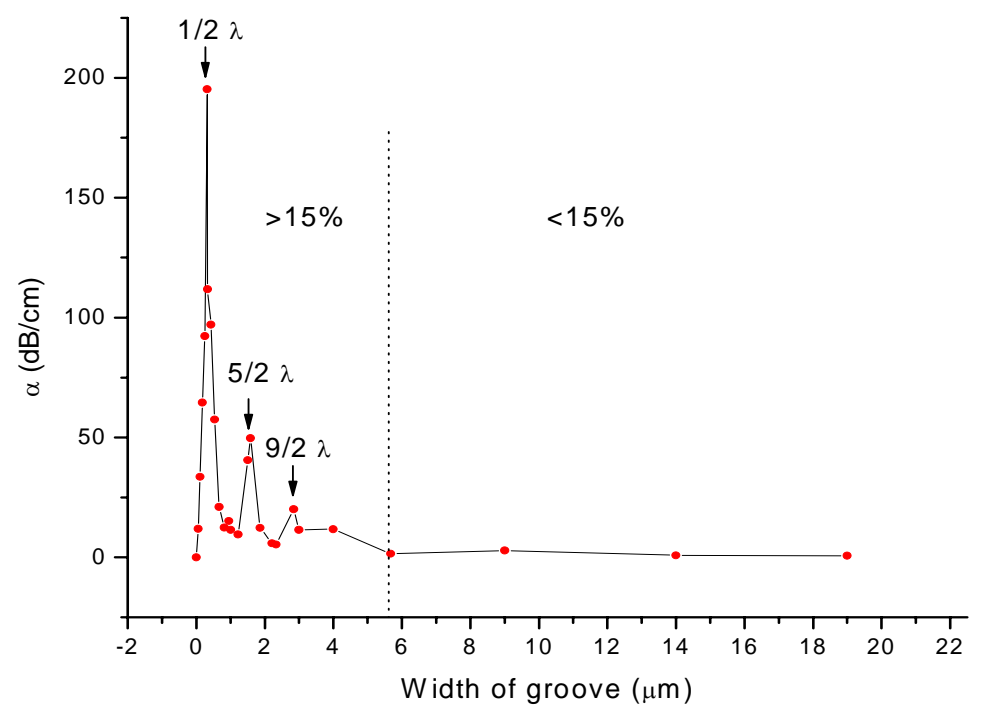

Fig. 5.16. Scattering coefficient vs. The distance between two isolated particles. 
For above graph (see Fig. 5.16), there are two regions, high scattering loss region (surface coverage $>15 \%$ ) and low scattering loss region (surface coverage $<15 \%$ ). There are some peaks in the high scattering loss region corresponding to large scattering loss. These peaks depend on wavelength and occur in $1 / 2 \lambda, 5 / 2 \lambda, 9 / 2 \lambda \ldots$ This is therefore most likely due to a grating like behavior arising from the surface attachment distributions. It must also be noted that when using VCM method to compute the scattering loss, we make an inherent assumption that the scattering loss is equal to the radiation power in groove. As a result this method may give large errors when applied under a large scattering loss condition. Thus, the scattering loss in this right surface loading region merits further research. In our experiments and in practical situations of value, the surface coverages are usually very small (i.e. several percent or fractions of a percent) and therefore the scattering loss is also small. Therefore the chosen model will be most accurate in this low coverage case, Fig. 5.17 shows the normalized scattering loss (assuming the total transmitting power equal to 1) caused by two isolated binding events for different interparticle distance and different particle size. Basically, for the same interparticle distance, a smaller particle has smaller scattering loss.

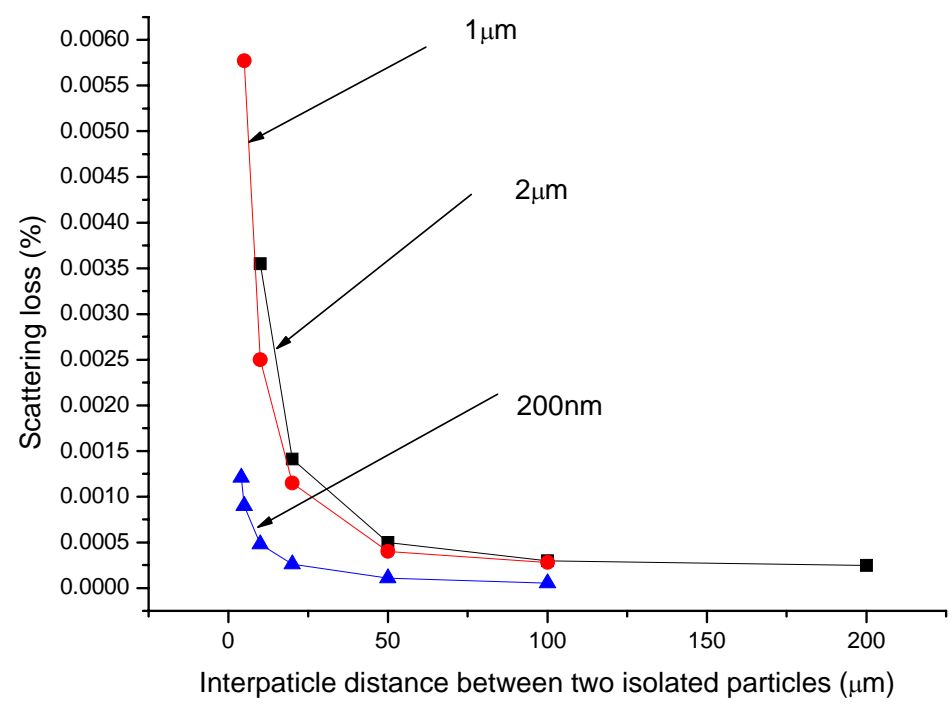

Fig. 5.17. Scattering coefficient vs. The distance between two isolated particles. 
In the low coverage region, the scattering loss between two binding events can be easily fitted as an exponential equation. If the scattering loss is small and the binding is periodic, the total scattering loss could be obtained by the following equation

$$
\text { Totalscattering }=\text { Fit } *(N-1)
$$

where Fit is fit equation and $N$ is the number of total particle which depends on percent coverage.

However, under normal condition, the binding is always random. That means the interparticle distance is random. To get total scattering loss, the distribution function should be applied. Here, we use normal distribution function

$$
P(x)=\frac{A}{\sigma \sqrt{2 \pi}} e^{-(x-\mu)^{2} / 2 \sigma^{2}}
$$

It satisfies (see Fig. 5-18)

$$
\int_{0}^{l} P(x) d x=1
$$

where $\mathrm{A}$ is normalized factor, $\sigma$ is the standard deviation, $\mu$ is the average interparticle distance.

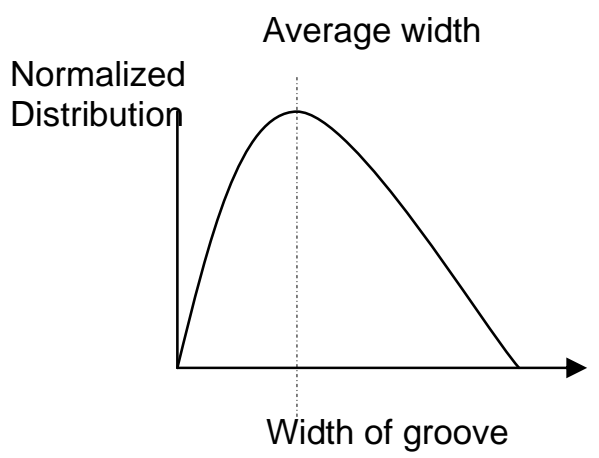

Fig. 5.18. Probability of distance between any two particles.

Thus, this normalized distribution function is used to approximate the total power loss in our waveguide caused by these random binding events (Eq. 5-59)

$$
\text { TotalScatteringLoss }=\int P(x) * \text { Fit } *(N-1)
$$

For a $1200-\mu \mathrm{m}$ waveguide, the total scattering loss corresponding to different coverage is shown as Fig. 5.19, 


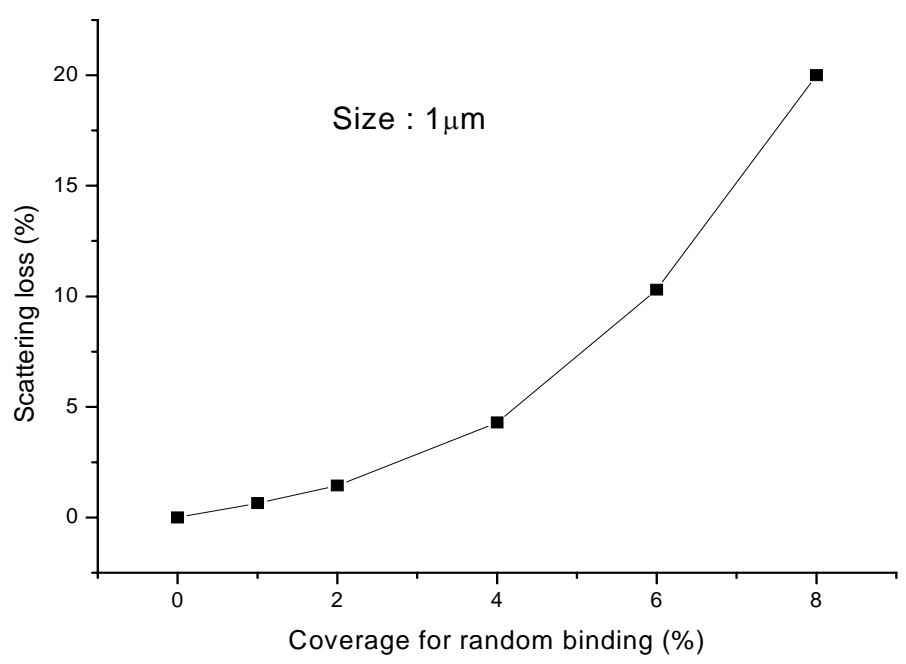

Fig. 5.19. Scattering loss vs coverage of binding events.

When the length of the waveguide is fixed, for a certain coverage but different size of binding events, the number of particles is different which results in the different scattering loss. If the size of the particle is larger than the penetration depth, for the same percent coverage, the smaller particle has larger scattering loss which is shown in Fig. 5.20. However, when the particle size is less than the penetration depth, the scattering loss will reach to maximum at a particular size. This behavior will be studied further in the future work.

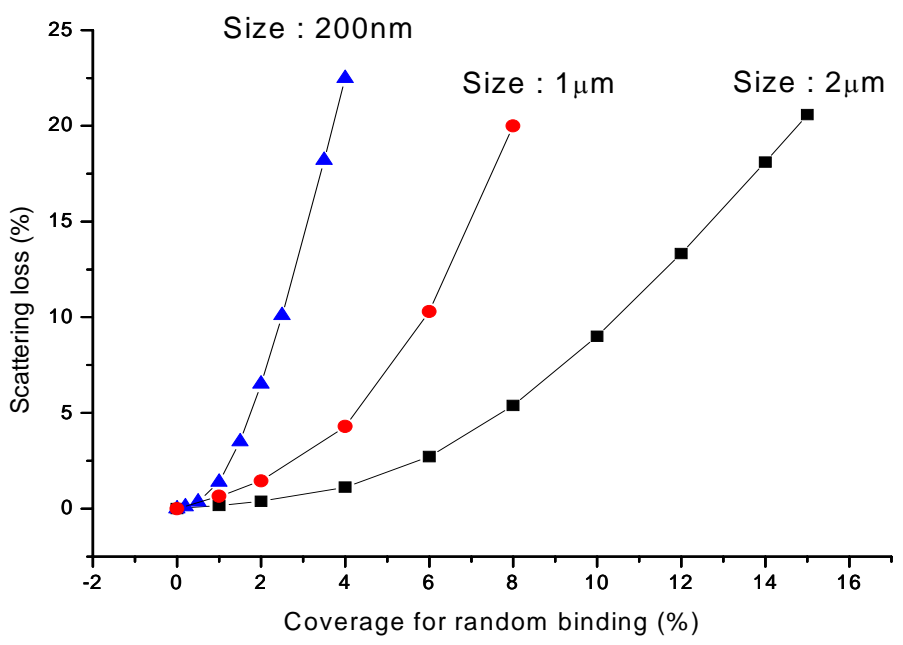

Fig. 5.20. Scattering loss VS coverage of binding events. 


\section{5-3 Issues Impacting the Limit of Detection (LOD) of the SPARROW biosensor}

To evaluate the issues impacting the SPARROW device's limit of detection, we consider here which factor dominates the change of light intensity at the end of the coupled waveguides. As we talked before, the final output will be influenced by the scattering loss, absorption loss and random binding events. Noise due to such influences as the optical source or random nonspecific surface interactions are not considered here but clearly of importance. These effects could be addressed in part through operation of a reference waveguide arm in the device and understanding of the statistical behavior of specific versus nonspecific binding events. Comparing other factors, the absorption loss and the scattering loss caused by material inhomogeneous could be neglected and moreover should be fixed during normal device operation. Also, before binding and after binding, the scattering loss caused by surface roughness is the same. Therefore, the changes of final output will depend on random binding events. Here the input power is normalized to one. Taking $\boldsymbol{S}$ as scattering loss caused by binding events and $\boldsymbol{P}$ as the bottom waveguide output power which is affected by the changes of the coupling condition caused by binding events, the LOD could be determined by $\boldsymbol{S}, \boldsymbol{P}$ and the limit

of intensity detector. Here, $\boldsymbol{S}$ always reduces the output power and the changes of $\boldsymbol{P}$ will depend on design structure. Fig. 5.21 shows how this power $\boldsymbol{P}$ changes with the index of the equivalent layer (cladding).

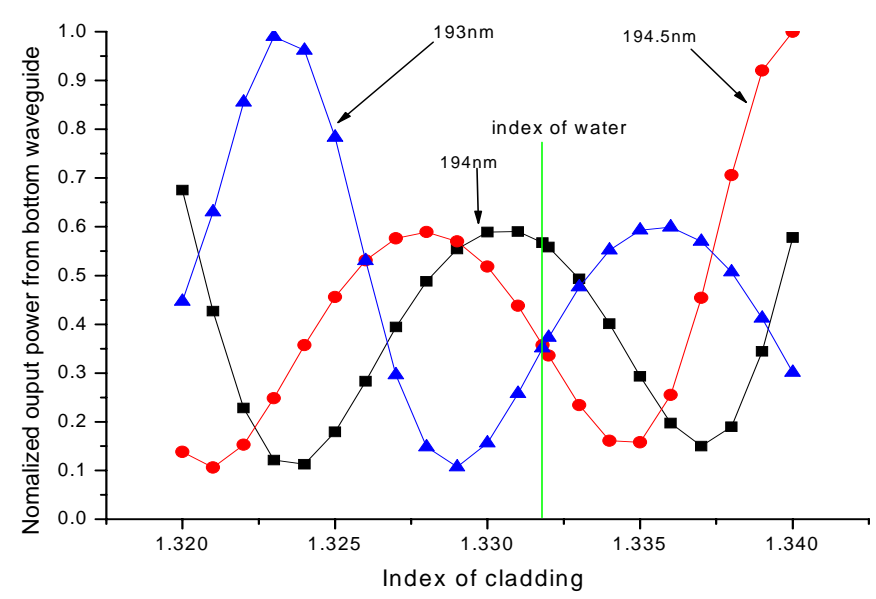

Fig. 5.21 Output intensity vs. index of equivalent layer 
$n=1.3318$

Waveguide $1 \boldsymbol{n}=1.659 \boldsymbol{d}=194 \mathrm{~nm}$

$n=1.457 d=1100 \mathrm{~nm}$

Waveguide $2 \boldsymbol{n}=1.659 \boldsymbol{d}=144 \mathrm{~nm}$

$n=1.4701$

Fig. 5.22 Ideal biosensor structure (wavelength 632.8nm)

In the ideal biosensor structure (Fig. 5.22), the operating point corresponding to the index of water $(\boldsymbol{n}=1.3318)$ above the top guide is located near the peak of a power oscillation. However, to achieve higher sensitivity, the operating point should be moved to the arm of the oscillation where the output power changes most rapidly with index. This could be accomplished by changing the thickness of top waveguide. Once the thickness of top waveguide changes from $194 \mathrm{~nm}$ to $193 \mathrm{~nm}$, the operating point is moved to the left side power oscillation maximum (Fig.5.21). In this case, when there are a few binding events such as latex beads which usually have larger refractive index (1.4 1.5) than that of water, the index of the equivalent layer will increase which results in an increase in $\boldsymbol{P}$. Under such condition, the LOD of the SPARROW biosensor requires

$$
|\Delta P-| S||>\text { limit of optical detection }
$$

where the LOD of the optical detector sensing the power in the bottom waveguide accounts for its noise sources. However, when the operating point is located on the right side of the maximum (for example, the thickness of top waveguide is equal to $194 \mathrm{~nm}$ or 194.5nm), very few binding events will lead to the output intensity decreasing. Now, the LOD should satisfy the relationship

$$
|\Delta P+| S||>\text { limit of optical detection }
$$

Obviously, this will greatly improve the sensitivity of the device.

Approximate numerical results were obtained by using the technique described above for both operating points described above. Assuming the size of particle is $40 \mathrm{~nm}$ 
and the refractive index of binding events is 1.5 , the $\Delta \mathbf{P}$ for different coverage could be obtained. (Fig. 5.23)

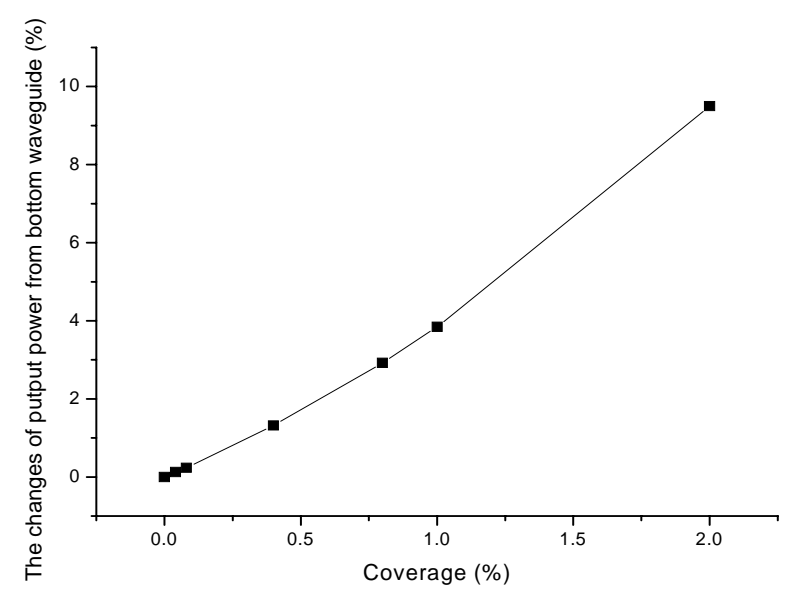

Fig. 5.23 The changes of output power from bottom waveguide as a result of random binding vs surface coverage

Before considering two operating points of interest, we address the issue of thickness sensitivity that is apparent from Figure 5.21. For a given set of optical waveguide stack materials refractive indices, the power oscillations in figure 5.21 are a function of the interaction length, here chosen to be $1200 \mu \mathrm{m}$. While a long interaction length has the desirable effect of increasing the rate of oscillation with refractive index, it has the negative effect of dramatically increasing the sensitivity of those oscillations to waveguide thickness. In general, a trade-off would be struck between sensitivity and achievable waveguide thickness control.

Now two operating cases are considered.

Operating point to the left of the power maximum: For a $1200-\mu \mathrm{m}$ waveguide interaction length, the output power from bottom waveguide will increase (as indicated in Fig.5.23) when there are some binding events. However, the scattering loss always reduces the total power in the waveguide (Fig.5.24). 


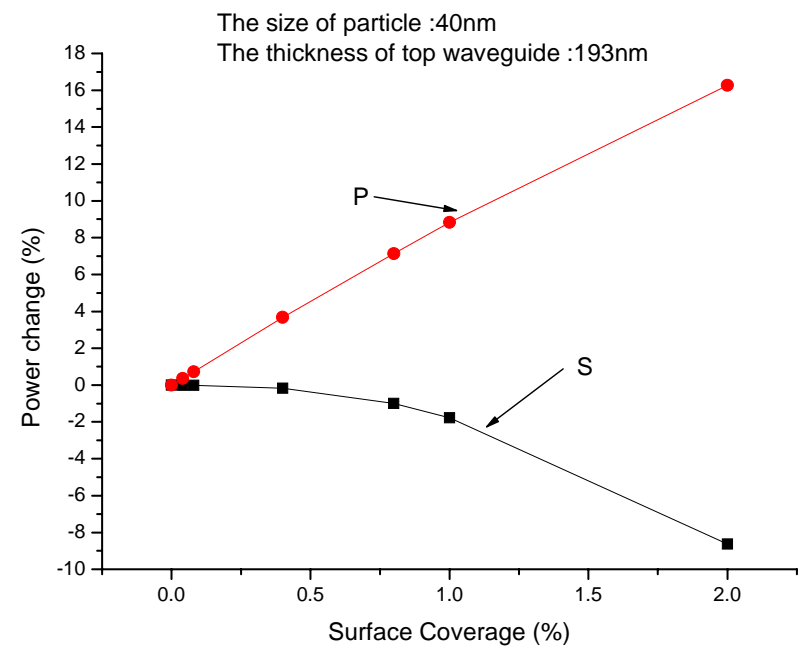

Fig. 5.24 The change of output power from bottom waveguide

Using Eq. 5-60, we have

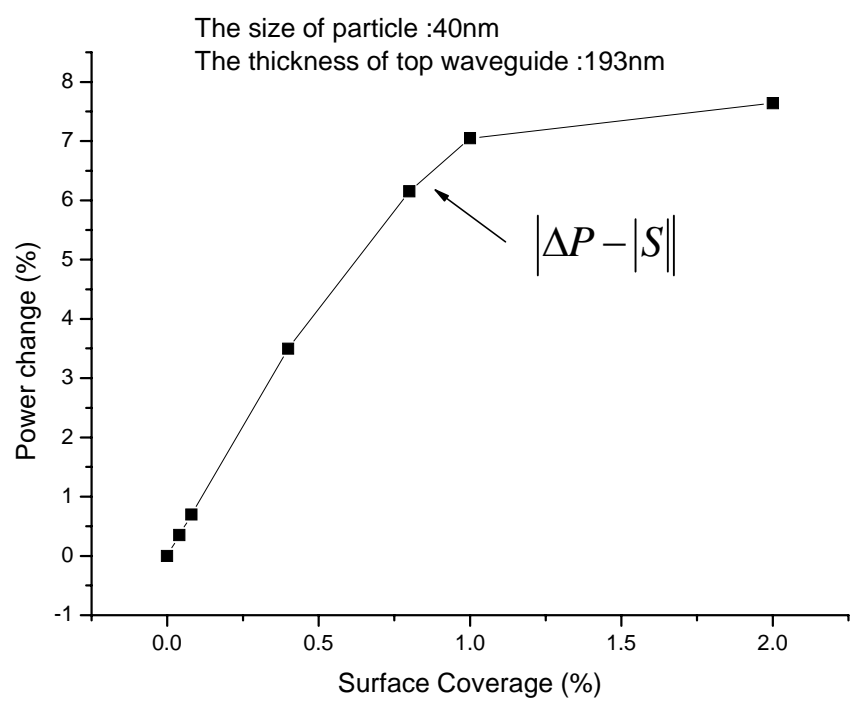

Fig. 5.25 Final changes of output power from bottom waveguide

If the minimum detectable change in optical power for our device is $1 \%$ of the total power at any given operating point, the minimum coverage for detection will be $0.112 \%$. Assuming the beads are anthrax spores and the mass density is about $0.081 \mathrm{~g} / \mathrm{mL}$ (Farquharson, et al., 2004), the LOD of this structure would be $3.62 \mathrm{pg} / \mathrm{mm}^{2}$. With high- 
percent coverage, the phases of both waveguides will totally mismatch, and sensitivity within this high coverage range is expected to be poor using the coupling phenomenon. Given power coupled into the lower waveguide prior to the flow cell interaction region will no longer couple to the upper waveguide, use of the upper waveguide would have to be achieved by direct input. Power that is launched into the upper probe waveguide will experience scattering dominated loss, perhaps useful in giving a direct measure of surface coverage in this high surface coverage regime.

Operating point to the right of the power maximum: Using a 1200- $\mu \mathrm{m}$ waveguide and the same beads as before, we only change the thickness of top waveguide from $194 \mathrm{~nm}$ to $194.5 \mathrm{~nm}$. Without binding, the normalized output power will be 35.7\%. Fig.5.26 shows the changes of output power versus coverage and Fig.5.27 shows final power changes.

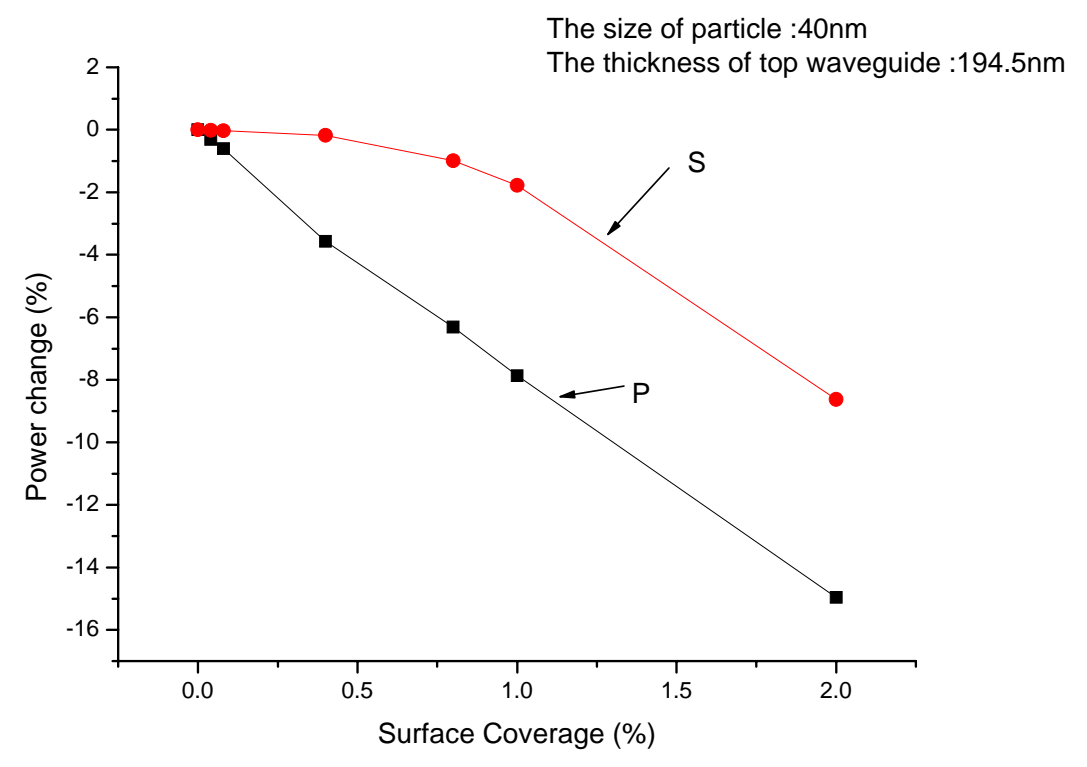

Fig. 5.26 The changes of output power from bottom waveguide 


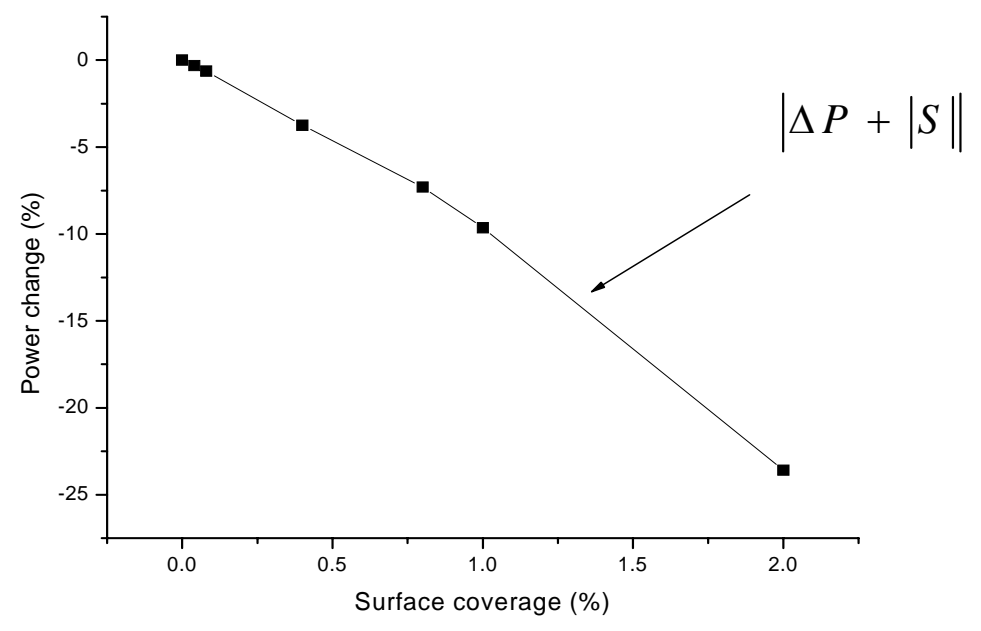

Fig. 5.25 Final changes of output power from bottom waveguide

Again, we set the minimum detectable change in optical power to $1 \%$ of the total power at a given operating point. Now, the minimum coverage for detection will be $0.108 \%$. Therefore, the LOD in this case will change to $3.51 \mathrm{pg} / \mathrm{mm}^{2}$. For different minimum detectable change in optical power and different sizes of particles, the LOD is shown in Fig.5.26.

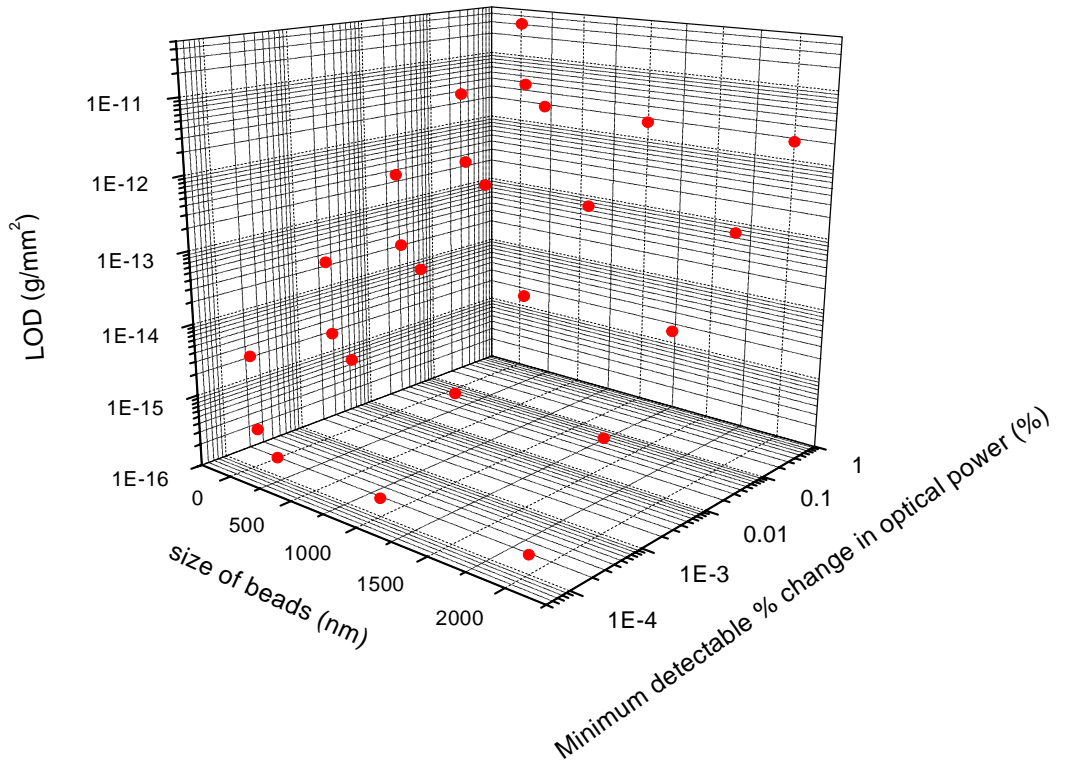

Fig. 5.26 LOD for different particles and different minimum detectable change in optical power 


\section{Chapter 6}

\section{Summary and discussion}

This dissertation work has led to the development of a mathematical model for the SPARROW biosensor, which is a particular type of sensing device based on a resonant optical-waveguide coupler composed of two waveguide layers. The mathematical approach adopted here used coupled mode theory (CMT). In addition, a commercially available computer simulation program (OptiBPM) based on a finite-difference beam propagation method (FD-BPM) was used. The simulation results were compared with experimental data obtained from fabricated SPARROW devices and with the CMT results.

For an ideal SPARROW biosensor model, the influence of waveguide thickness and waveguide index variation on biosensor behavior was investigated. Also, a numerical evaluation was performed of the resulting changes in output light intensity for both waveguides that occur when a biolayer is coated on the waveguide surface. The results indicate that the biolayer's existence will greatly affect the coupling length and coupling efficiency of the resonant waveguide coupler.

To enable better simulation of actual biosensor behavior, the biolayer construction and experimental data were also included in this dissertation work. Using these data, a non-ideal biosensor model was developed. In the non-ideal model, power scattering losses in waveguides caused by surface roughness and material index fluctuations were included. Additionally, for random binding events on the biosensor surface, the dependence of the coupling lengths and coupling efficiency on percent coverage were determined. Based on this model, a prediction of the output light intensity of the biosensor can be obtained. Based on the modeling work, experimental measurement of light output intensity can now be related to a percentage of surface loading. The main highlights of this dissertation work are listed below.

- Established an analytical model for the ideal SPARROW biosensor, allowing optimization of both thickness and optical constants of each layer. 
- Determined the range of behaviors with different effective indexes of the biolayer.

- A biolayer appropriate for the SPARROW biosensor was designed and constructed. The quality and stability of such a layer under actual device operation conditions was experimentally measured (under supervision of personnel working in WVU Chemistry Department).

- Established an analytical model to describe the non-ideal SPARROW biosensor case (i.e., real, practical devices) which then led to predictions of the influence of surface roughness of both waveguides and biolayers on waveguide coupling and losses. Also, the non-ideal case was extended to include the effects of random binding events on output intensity.

- Establish models to find the transition between scattering behavior arising from a few binding events on the surface, and the onset of detectable coupling changes when enough target material is present.

The analytical models developed here are not only suitable for SPARROW biosensor studies, but also can be applied to other kinds of optical devices which are based on directional couplers. However, the application range has some limitations. First, in both ideal and non-ideal cases, it is assumed that there is only a single TE mode in each waveguide. Thus, the index and thickness of waveguide have to be considered. Second, since perturbation theory was used in the non-ideal case, the variations of thickness and index have to be limited to a small value to avoid large error in our numerical results. Third, because the size of general binding events is much larger than the waveguide thickness, our scattering loss model VCM works well only if we have a small index contrast between binding events and cladding layer. If the index contrast is large, the optical mode in the waveguide will be more complex and an analytical expression for scattering loss would not be obtainable. This is also in accord with the initial assumption for both CMT and FD-BPM: assuming the beam amplitude in the waveguide varies slowly with distance so that we can cancel the $\frac{\partial^{2} a_{m}}{\partial z^{2}}$ term in the coupled waveguides propagation equation. 
Compared to computer simulations, the analytical model shows good agreement. More experimental work is needed to test the analytical model. Future work should address further validation of the model. Compared to experimental testing, the analytical model offers an easy way to evaluate and predict the device behavior once it is fully validated by experiment. In addition, the most model will help significantly to guide our device optimization. 
APPENDIX I: Analytical model computing program (using Mathematica version 5.0, by Wolfram Research)

Program I: Computation of coupling lengths and coupling efficiency

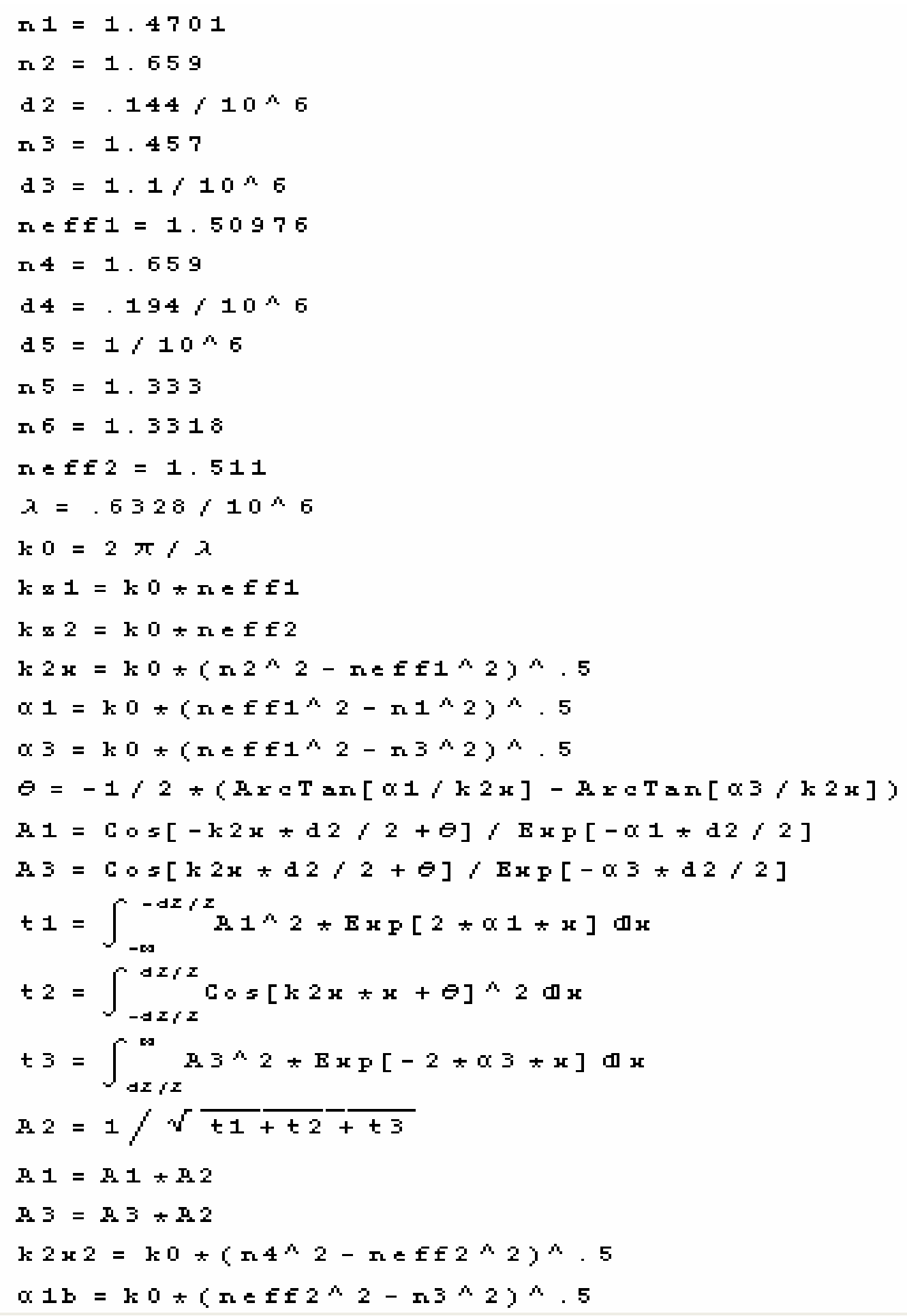




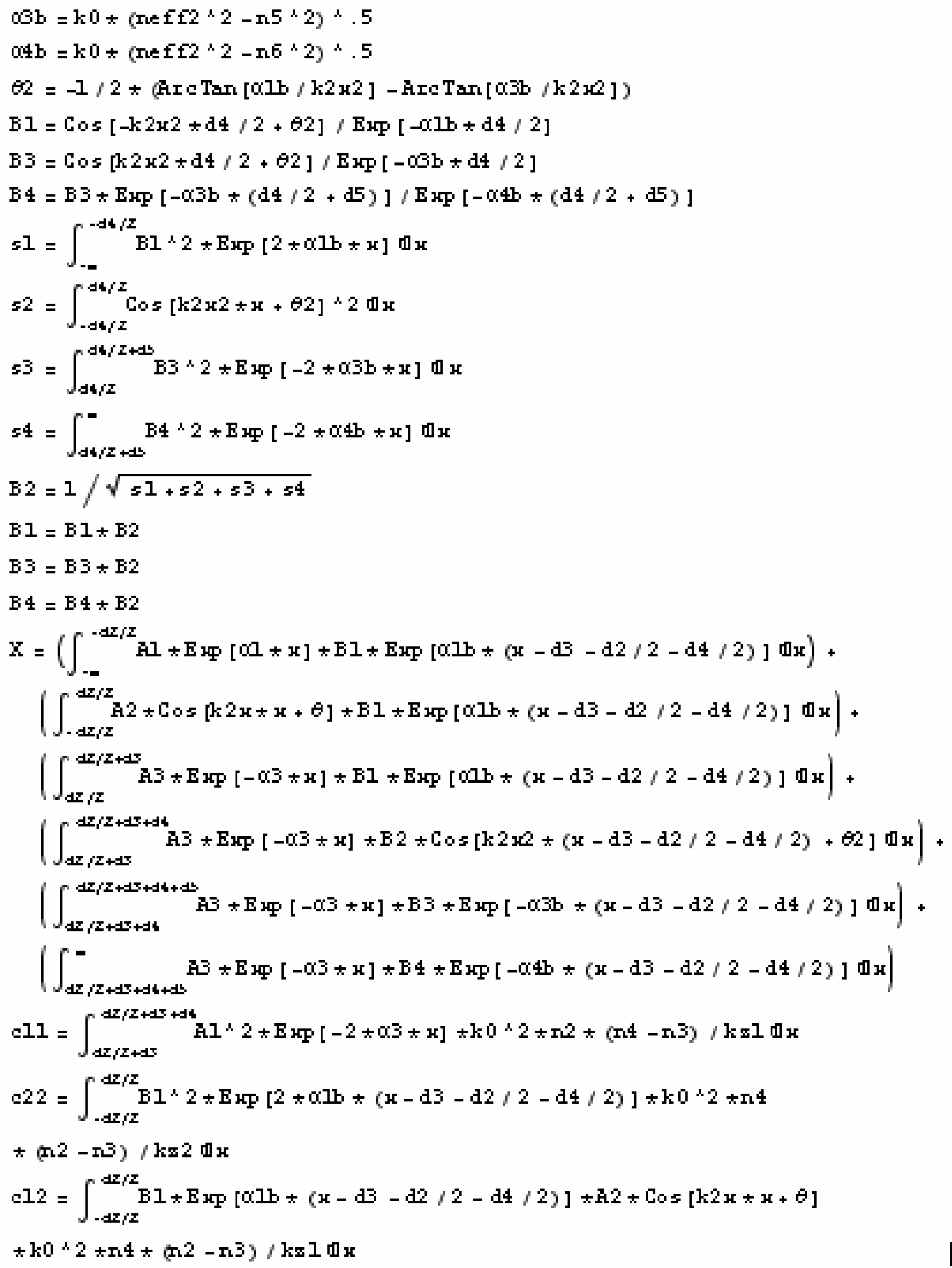




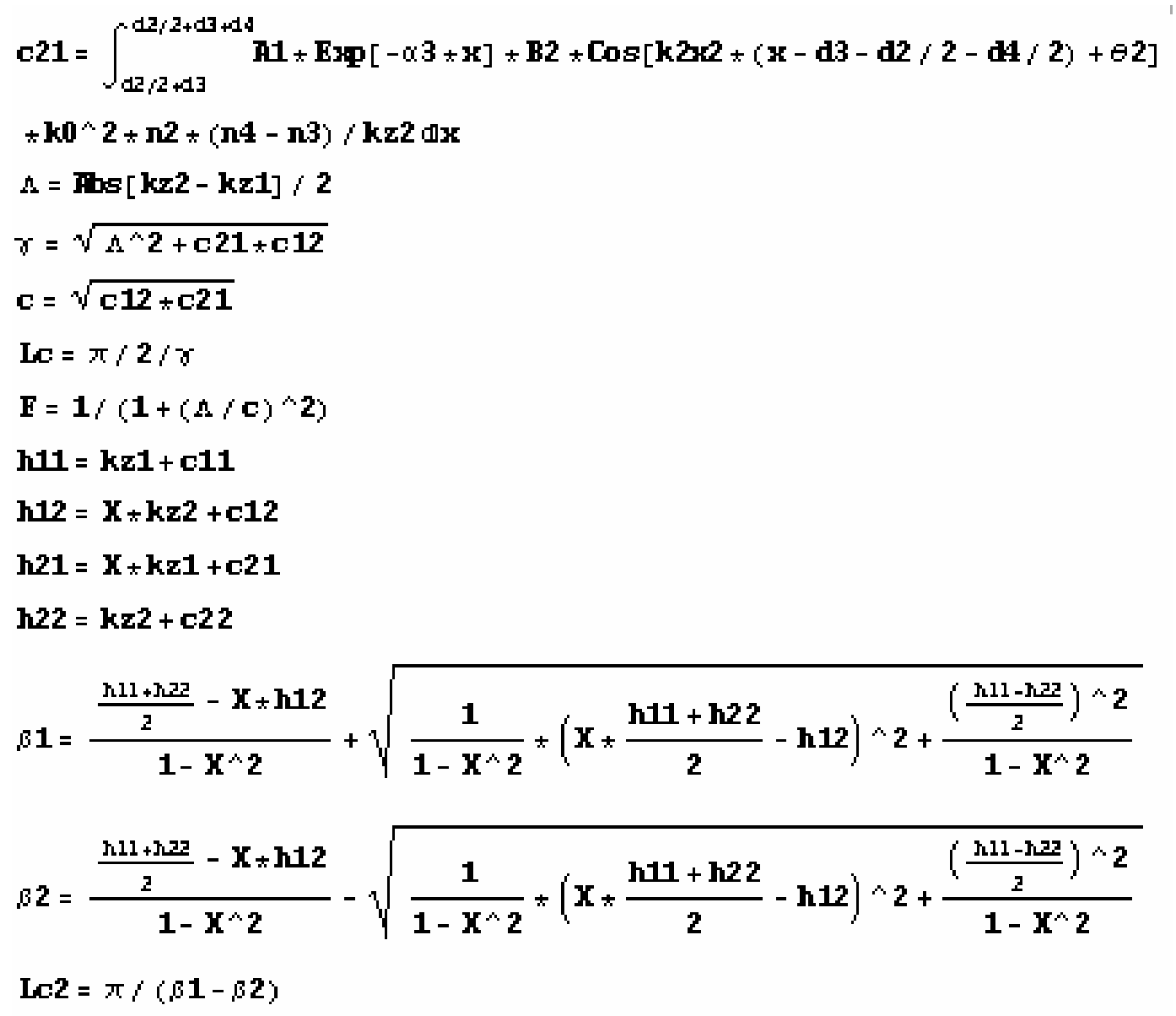


Program II: Scattering loss due to surface roughness - results are in units of $\mathrm{dB} / \mathrm{cm}$.

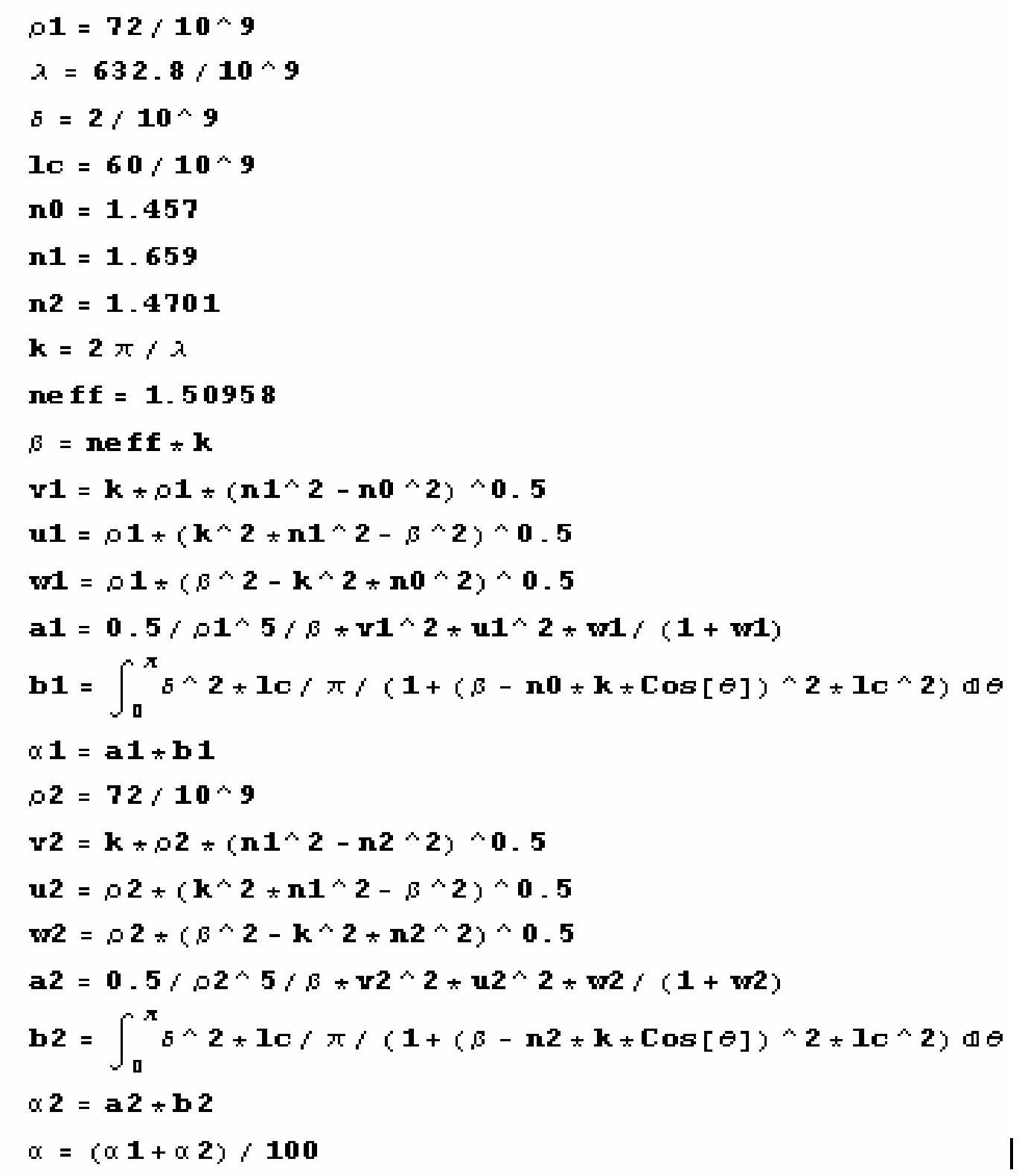

$\boldsymbol{\rho} 1$ - the half width of waveguide $\quad \lambda$-the wavelength of HeNe laser (light source)

$\boldsymbol{\delta}$-RMS surface roughness $\quad \boldsymbol{l} \boldsymbol{c}$-correlation length

$\boldsymbol{n 0}$ - the refractive index of substrate $\quad \boldsymbol{n} \mathbf{l}$-the refractive index of waveguide

$\boldsymbol{n} \mathbf{2}$ - the refractive index of cladding $\quad \boldsymbol{k}$ - the wavevector in vacuum

neff - the effective index of waveguide

$\boldsymbol{a}$ - the scattering loss of waveguide $(\mathrm{dB} / \mathrm{cm})$ 


\section{References}

Allara, D.L., Nuzzo, R.G., Spontaneously organized molecular assemblies. 1. formation, dynamics, and physical properties of $n$-Alkanoic acid adsorbed from solution on an oxidized aluminum surface. Langmuir, V1, p45-52, 1985

Amanda J. Haes, Richard P., Van Duyne. A Highly Sensitive and Selective SurfaceEnhanced Nanobiosensor. Mat. Res. Soc. V723, O3.1.1-3.1.6, 2002

Ames, G.H., Hall, D.G., Attenuation in planar optical waveguides: comparison of theory and experiment, IEEE, Journal of Quantum Electronics, Vqe-19, n5, p845-853, 1983

Anal. Chem., Enzyme-Based Fiber Optic Sensor. American Chemical Society. V57, p565-566, 1985

Anderson, G.P., Rowe-Taitt, C.A., Ligler, F.S., Raptor: A portable, automated biosensor. Photonic detection and intervention technologies for safe food proceedings. V4206, 2001

Avidin-Biotin Chemistry: A handbook. Part 1: Components of Avidin-Biotin Technology. Pierce. Chemical Company, Rockford, 1994

Baumann, S. M., Martner, C. C., Martin, D. W., Blattner, R. J., Braundmeier, A. J., A study of electron beam evaporated $\mathrm{SiO}_{2}, \mathrm{TiO}_{2}$, and $\mathrm{Al}_{2} \mathrm{O}_{3}$ films using RBS, HFS, and SIMS. Nuclear Instruments and Methods in Physics Research. B45, n1-4, p664-668, 1990

Biddle, M.M., An Introduction to SAMs: Self-Assembled Monolayers in Organic Chemistry. 2001. http://www.chem.wisc.edu/areas/organic/studsemin/biddle/biddleabs.pdf

BIO-WISE web site. BIO-WISE BIOTECHNOLOGY AT WORK, Biosensors for 
industrial applications: A review of biosensor technology. 2001, Original from www.biowise.org.uk (closed). Currently in http://www.ecdti.co.uk/CGIBIN/priamlnk.cgi?MP=CATSER\%5EGINT65\&CNO=1\&CAT='52'

Boisde, G., Harmer, A., Chemical and biochemical sensing with optical fibers and waveguides. Artech House Publishers, Boston · London, 1996.

Chimenti, D.E., Lobkis, O.I., Effect of rough surfaces on guided waves in plates. Ultrasonics, V36, n1, p155-162, 1998

Choi, M.F., Progress in Enzyme-Based Biosensors Using Optical Transducers. Microchimica Acta. V148, n3-4, p107-132, 2004

Clark, L.C., Lyons, C., Electrode systems for continuous monitoring in cardiovascular surgery. Ann. NY Acad. Sci. 102: p29-45, 1962

Ctyroky, J., Theory and modeling of optical waveguide sensors utilizing surface plasmon resonance. Sensors and actuators, B54, p66-73, 1999

Dai, Y.J., Lin, L., Li, P.W., Chen, X., Wang, X.R., Wong, K.Y., Comparison of BOD optical fiber biosensors based on different microorganisms immobilized in ormosil matrixes. International Journal of Environmental and Analytical Chemistry, V84, n8, p607-617, 2004

Devaprakasam, D., Sampath, S and Biswas, SK, Thermal stability of perfluoroalkyl silane self-assembled on a polycrystalline aluminum surface. Langmuir, V20, p13291334,2004

Diamond, D., Principle of chemical and biological sensors. Chemical analysis, vol. 150, John Wiley \& Sons, Inc, 1998 
Dzyadevych, S.V., Amperometric biosensor for ethanol detection based on alcohol oxidase immobilized within electrochemically deposited Resydrol film. Materials Science \& Engineering C, Biomimetic and Supramolecular Systems, V26, n2-3, 2006

Energy Science News: New cancer biosensor. 2001 http://www.pnl.gov/energyscience/06-01/brf.htm

Farquharson, S., Grigely, L., Khitrov, V., Smith, W., Sperry, J.F., Fenerty, G., Detection Bacillus cereus spores on a mail sorting system using Raman spectroscopy. Journal of Raman spectroscopy. V35, p82-86, 2004

Flory, C.A, Analysis of directional grating-coupled radiation in waveguide structure. IEEE, Journal of Quantum Electronics, V40, n7, p949-957, 2004

Garcés, I., Villuendas, F., Salinas, I., Alonso, J., Puyol, M., Domínguez, C., Llobera, A., Analysis of optochemical absorbance sensors based on bidimensional planar arrow microoptics. Sensors and Actuators, B 60 (2-3), p191-199, 1999

Garcia-martin A. Saenz, J.J., Statistical properties of wave transport through surfacedisordered waveguides. Wave in random and complex media, V15, n2, p229-268, 2005

Griffel, G., Coupled mode formulation for directional couplers with longitudinal perturbation. IEEE, Journal of Quantum Electronics, V27, n4, p985-994, 1991

Grant, S.A., Sungho, Ko., A novel FRET-based optical fiber biosensor for rapid detection of Salmonella typhimurium. Biosensors \& Bioelectronics, V21, n7, p1283-1290, 2006

Hall, D. G., Coupled mode theory for corrugated optical waveguides. Optics letters, V15, n11, p619-621, 1990

Hardy, A.A., Streifer, W.G., Osinski, M., Weak coupling of parallel waveguides. Opt. Lett. 
V13, p161-163, 1988

Haus, H. A., Huang, W., Coupled-mode theory. Proc. IEEE. V79, p1505-1518, 1991

Haus, H.A., Huang, W., Kawakami, S., Whitaker, N.A., Coupled mode theory of optical waveguides. Journal of Lightwave Technology. V5, n1, p16-23, 1987

Haus, H.A., Huang, W., Snyder, A.W., Coupled mode formulations. Opt. Lett. V14, p1222-1224, 1989

Huang, X., Nausieda, I., Greve, D.W., Domach, M.M., Nguyen, D., Development of active matrix biosensor array for cell screening. Proceedings of the IEEE Sensors 2004 V1, p72-75, 2004

Huang, W., Coupled mode theory for optical waveguides: an overview. Opt. Soc. Am. A V11, n8, p963, 1994

Huang, W., Hong, J., A new approach to grating assisted couplers. Journal of Lightwave Technology. V9, n6, p721-727, 1991

Huang, W., Lit, J.W.Y., Nonorthogonal coupled mode theory of grating assisted codirectional couplers. Journal of Lightwave Technology. V9, n7, p845-852, 1991

Imai, M., Miyanaga, S. Asakura, T., Mode conversion and radiation loss caused by refractive-index fluctuations in an asymmetric slab waveguide. IEEE Journal of Quantum Electronics, VQE13, n4, p255-262, 1977

Ishimaru, A., Wave propagation and scattering in random media and rough surface. Proceedings of the IEEE, V79, n10, p1359-1366, 1991 
James, P. J., Antognozzi, M., Tamayo, J., McMaster, T.J., Newton, J.M., Miles, M.J., Interpretation of contrast in tapping mode AFM and shear force microscopy. A study of nafion., Langmuir, V17, p349-360, 2001

Jin, G.H., Harari, J., Joannes, L., Vilcot, J.P., Decoster, D., Numerical analysis of the radiation losses due to surface roughness in integrated optics devices. IEEE photonic tech. letters, V8, n9, p1202-1204, 1996

Johnston, A., Trends in biosensor research and development. Electron Devices Meeting, Technical Digest., p654-657, 1998.

Kelly, D., Grace, K.M., Integrated optical biosensor for detection of multivalent proteins. Optics letters. V24, n23, p1723-1725, 1999

Killard, A.J., Smyth, M.R., Grennan, K., Micheli, L., Palleschi, G., Rapid antibody biosensor assays for environmental analysis. Biochem Soc Trans. V28, n2, p81-p84, 2000

Kissinger, P.T., Introduction to Amperometric biosensor configurations. www.currentseparations.com , 2006

Kogelnik, H., Ramaswamy, V., Scaling Rules for Thin-Film Optical Waveguides, applied physics, V13, n8, p1857, 1974

Kosuri, M.R., Cone, R., Li, Q., Han, S.M., Bunker, B.C., Mayer, T.M., Adsorption kinetics of 1-alkanethiols on Hydrogenated Ge (111), Langmuir, V20, n3, p835-840, 2004

Kress-Rogers, E., Handbook of biosensors and electronic noses: Medicine, food and environment. CRC press, Boca Raton, LA. Chapter 3, 1997

Kumar, A., Biosensors Based on Piezoelectric Crystal Detectors: Theory and Application. JOM, The Minerals, Metals \& Materials Society, V52, n10, 2000 
Lacey, J. P. R., Radiation loss from planar waveguides with random wall imperfections, IEE proceedings. V137. n4, p282-288, 1990

Ladouceur, F., Effect of side wall roughness in buried channel waveguides. IEE Proc.optoelectron., V141, n4, p242-248, 1994

Ladouceur, F., Effect of roughness and inhomogeneity on evanescent single mode optical couplers. IEE Proc.-optoelectron., V142, n6, p288-292, 1995

Ladouceur F., Poladian, L., Surface roughness and backscattering. Optics letters. V21, n22, p1833-1835, 1996

Ladouceur, F., Roughness, Inhomogeneity, and Integrated Optics. Journal of Lightwave Technology. V15, n6, p1020-1025, 1997

Lee, D.L., Electromagnetic Principles of Integrated Optics. John Wiley \& Sons, 1986.

Liang, A.H., The Rayleigh Scattering Loss of Isotropic Weakly Guiding Single-Mode Waveguides. IEEE Journal of Quantum Electronics. V28, n9, p1844-1847, 1992.

Lim M. S., Feng, K., Chen, X., Wu, N., Raman, A., Gawalt, E.S., Nightingale, J., Korakakis, D., Hornak, L., and Timperman, A., Adsorption and Desorption of Stearic Acid Self-assembled Monolayer on Aluminum Oxide, submitted to Langmuir, 2006

Little, B. E., A variation coupled-mode theory including radiation loss for grating-assisted couplers. Journal of Lightwave Technology. V14, n2, p188-195, 1996

Lloyd, D., Hornak, L., Pathak,S., Morton, D., Stevenson, I., Application of Ion Beam Assisted Thin Film Deposition Techniques to the Fabrication of a Biosensor Chip with Fieldability Potential for Important Biohazard Detection Applications. Society of 
Vacuum Coaters 505/856-7188. 47th Annual Technical Conference Proceedings 2004 Luff, B.J., Harris, R.D., Wilkinson, J.S., Wilson, R., Schiffrin, D.J., Integrated-optical directional coupler biosensor. Optics letters. V21, n8, p618-620, 1996

London south bank university, www.lsbu.ac.uk/biology/enztech/potentiometric.html

Luff, B.J., Wilkinson, J.S., Piehler, J., Hollenbach, U., Ingenhoff, J. and Fabricius, N., Integrated optical Mach-Zehnder biosensor. Journal of Lightwave Technology. V16, n4, p583-592, 1998

Mac Craith, B. D., Mcdonagh, C., O"Keeffe, G., Butler, T., O"Kelly B., Mcgilp, J. F., Fibre optic chemical sensors based on evanescent wave interactions in sol-gelderived porous coatings. Journal of Sol-Gel Science and Technology. V2, n1-3, p661-665, 1994

Maes, H. E., Claeys, C., Mertens, R., Campitelli, A., Van Hoof, C., De Boeck, J., Trends in microelectronics, optical detectors, and biosensors, advanced engineering materials. n10,p781-787, 2001

Magonov, S.N., Elings, V., Whangbo M.-H., Phase imaging and stiffness in tapping mode atomic force microscopy. Surface science letters, V375, n2-2, p385-391, 1997

Marcatili, E., Improved coupled mode equations for dielectric guides. IEEE J. Quantum Electron. VQE-22, p988-993, 1986

Marcuse, D., Integrated optics. IEEE Press, 1973

Marcuse, D., Radiation loss of grating-assisted directional coupler. IEEE, Journal of quantum electronics, V26, n4, p675-684, 1990 
Marcuse, D., Theory of dielectric optical waveguides. Academic, New York, 1991

Miller, S.E., Coupled wave theory and waveguide applications. Bell Syst. Tech. J V33, 1954

Nagel, B., Dellweg, H., Gierasch, L.M., Glossary for chemists of terms used in biotechnology, Pure \& Appl. Chem., V64, n1, p148, 1992

Newman, J.D., Tigwell, L.J., Warner, P.J., Turner. A.P.F., Biosensors: Boldly going into the new millennium. Sens. Rev., 21(4), p268-271, 2001

Nightingale, J., Cornell, T., Samudrala, P., Poloju, P., Hornak., L., Korakakis, D, Reactive Deposition of dielectrics by beam assisted E-Beam Evaporation. Submitted, 2006

Ogilvy, J. A., Rough surfaces: Gaussian or exponential statistics? J. Phys. D: Appl. Phys 22, p1243-1251, 1989

OptiBPM manual: technical background and tutorials. Version6.0, Optiwave Corporation, Ottawa, ON, Canada

Optiwave manual: technical background and tutorials. Version4.0, Optiwave Corporation, Ottawa, ON, Canada

Palais, J.C., Fiber Optic Communications. Prentice Hall, April, 2002

Passaro, V.M.N., Analysis of radiation loss in grating assisted codirectional couplers. IEEE J. quantum electronics, V31, n9, p1691-1697, 1995

Patton, J.C., Overview of biosensor design and fabrication. Images of the Twenty-First Century. Proceedings of the Annual International Conference of the IEEE Engineering in Medicine and Biology Society (Cat. No.89CH2770-6), V4, p1081, 1989 
Pouthas, F., Gentil, C., Côte, D., Bockelmann U., DNA detection on transistor arrays following mutation-specific enzymatic amplification. Applied physics letters V84, n9, p1594-1596, 2004

Powner, E.T., Yalcinkaya F.1., Intelligent Biosensors. Sens. Rev., 17(2), p107-116, 1997

Preininger C., Danielsson, B., Thermometric determination of copper(II) using acid urease. The Analyst. V121, n11, p1717-1720, 1996

Ramsay, G.., Commercial biosensors applications to clinical bioprocess and environmental samples, Chemical analysis, vol. 148, John Wiley \& Sons, Inc., 1998

Rigrod, W.W., Marcuse, D., Radiation loss coefficient of asymmetric dielectric waveguides with shallow sinusoidal corrugations. IEEE J. quantum electronics, VQE-12, n11, p673-685, 1976

Riihe, J., Novotny, V.J., Kanazawa, K.K, Clarke, T., Street, G.B, Structure and tribological properties of ultrathin alkylsilane films chemisorbed to solid surface. Langmuir, V9, p2383-2388, 1993

Sahney, R., Anand, S., Puri, B.K., Srivastava, A.K., A Comparative study of immobilization techniques for urease on glass-PH-electrode and its application in urea detection in blood serum. Analytica Chimica Acta, V578, n2, p156-161, 2006

Sampath, S., Lev, O., 3D organized self-assembled monolayer electrodes: A novel biosensor configuration. Advanced materials, V9, n5, p410-413, 1997

Sangeeta, S., Sharma, E. K., Analytical expressions for effective indices in a threewaveguide coupler. Opt. Lett. V20, p1005, 1995 
Schiestel, T., Brunner, H., Tovar, G.E.M., Controlled surface functionalization of silica nanospheres by covalent conjugation reactions and preparation of high density streptavidin nanoparticles. J. Nanoscience and Nanotechnology, V4, n5, p504-511, 2004

Shankaran, D.R., Matsumoto, K., Toko, K., Miura, N., Development and comparison of two immunoassays for the detection of 2,4,6-trinitrotoluene (TNT) based on surface plasmon resonance. Sensors and Actuators B. V114, n1, p71-79, 2006

Shieh, S., An Analysis of Contact Angle Measurement. 2001. http://www.p2pays.org/ref/13/12920.htm

Shriver, L.C., Ligler, F.S., The array biosensor for counterterrorism. IEEE sensors journal, V5, n4, p1-6, 2005

Silverstein, R.M., Bassler, G.C., Morrill, T.C., Spectrometric Identification of Organic Compounds, 4th edition. New York: John Wiley \& Sons, p166, 1981

Singapore's Institute Of Bioengineering And Nanotechnology web site http://www.biomedsingapore.com/bms/sg/en_uk/index/newsroom/pressrelease/0/singapore_s_institute.html

Snyder, A.W., Love J.D., Optical waveguide theory. Chapman \& Hall, London, 1988

Streifer, W., A critical review of coupled mode theory. Poc. Soc. Photo-opt. Instum. Eng. 835, p178-187, 1987

Streifer, W., Coupled mode theory. Electron. Lett. V23, n7, p315-316, 1988

Tao, Y.T., Structure comparison of self-assembled monolayers of n-alkanoic acids on surfaces of silver, copper, and aluminum. J. Am. Chem. Soc. P4350-4358, 1993

Taylor, C. E., Schwartz, D. K., Octadecanoic acid self-assemble monolayer growth at sapphire surfaces. Langmuir V19, 2665-2672, 2003

Terry, L.A., White S.F., Tigwell L.J., The Application of Biosensors to Fresh Produce and 
the Wider Food Industry. J. Agric. Food Chem., V53, n9, p1309-1316, 2005

Thompson, R.B., Jones, E.R., Enzyme-Based Fiber Optic Zinc Biosensor, Anal. Chem. American Chemical Society, V65, p730-734, 1993

Trillo, S., Wabnitz, S., Nonlinear nonreciprocity in a coherent mismatched directional coupler. Appl. Phys. Lett. V49, n13, p752-754, 1986

Tseng, S. M., Zhan, J., Derivation of coupling coefficients of modes in slab coupler: Ray theory. J. Appl. Phys. V38, n4a, p1989-1993, 1999

Turner, A.P.F., Karube, I., Wilson,G. S., Biosensors: Fundamentals and Applications. Oxford University Press, Oxford. p770, 1987

Ulman, A., An introduction to ultrathin organic films. Academic press. Inc. New York, 1991

Ursula .E. Spichiger-Keller, Chemical Sensors and Biosensors for Medical and Biological applications. WILEY-VCH, 1998

Vassello, C., Condensed formula for coupling coefficients between parallel dielectric waveguides, Electron. Lett. V23, p334-335, 1986

Vassello, C., About coupled mode theories for dielectric waveguides. Journal of Lightwave Technology. V6, n2, p294-303, 1988

Vianello, F., Cambria, A., Ragusa, S., Cambria M.T., Zennaro, L., Rigo, A., A high sensitivity amperometric biosensor using a monomolecular layer of laccase as biorecognition element. Biosensors \& bioelectronics. V20, n2, p315, 2004

Wang, L., etc., Electromagnetic wave propagation in and scattering from random media. Applied physics letter. V72, n15, p1914, 1998 
West, L.C., Roberts, C., Dunkel, J., Wojcik, G., Mould, J., Non uniform grating couplers for coupling of Gaussian beams to compact waveguides. Integrated Photonic Research Technical digest, 1994

Yamakita, J., Rokushima, K., Scattering losses caused by irregular boundary interfaces and refractive index fluctuations in a thin-film waveguide. The transactions of the IEIC of Japan. VE63, n8, p588-589, 1980

Yariv, A., Coupled-mode theory for guided-wave optics. IEEE. J. Quantum Electron VQE-9, p919-933, 1973

Young, P.R., Collier, R. J., Exact solution of lossy asymmetrical coupled dielectric slab waveguides. 31st European Microwave Conference, London, 2001.

Yuk, J. S., Ha, K. S., Proteomic applications of surface plasmon resonance biosensors: analysis of protein array. Experimental and molecular medicine, V37, n1, p1-10, 2005 